\title{
CHECKLIST OF SPIDERS (ARACHNIDA: ARANEAE) OF SOUTH ASIA INCLUDING THE 2006 UPDATE OF INDIAN SPIDER CHECKLIST
}

\author{
Manju Siliwal ${ }^{1}$ and Sanjay Molur ${ }^{2,3}$ \\ 1,2 Wildlife Information \& Liaison Development (WILD) Society, ${ }^{3}$ Zoo Outreach Organisation (ZOO) \\ 29-1, Bharathi Colony, Peelamedu, Coimbatore, Tamil Nadu 641004, India \\ Email: ${ }^{1}$ manjusiliwal@rediffmail.com; ${ }^{3}$ herpinvert@vsnl.com
}

\begin{abstract}
After one year since publication of the Indian Checklist, this is an attempt to provide a comprehensive checklist of spiders of South Asia with eight countries - Afghanistan, Bangladesh, Bhutan, India, Maldives, Nepal, Pakistan and Sri Lanka. The Indian checklist is also updated for 2006. The South Asian spider list is also compiled following The World Spider Catalog by Platnick and other peer-reviewed publications since the last update. In total, 2299 species of spiders in 67 families have been reported from South Asia. There are 39 species included in this region's checklist that are not listed in the World Catalog of Spiders. Taxonomic verification is recommended for 51 species. Changes in the Indian spider checklist have been highlighted in this paper. The paper lists emendations, extralimital distribution, incertae sedis, new combinations, nomina nuda, nomina dubia, transfers, synonyms, etc. The checklist is updated up to 31 December 2006.
\end{abstract}

\section{KEYWORDS}

Afghanistan, Arachnida, Araneae, Bangladesh, Bhutan, checklist, India, Maldives, Nepal, Pakistan, South Asia, spiders, Sri Lanka

Since the publication of the comprehensive updated checklist of Indian spiders (Siliwal et al., 2005), there has been an incredible increase in the number of spider fauna all over the world -500 new species, 40 new genera and one resurrected family; the total now standing around 39500 species in 3642 genera and 111 families (Platnick, 2006). In keeping with the objectives set out last year, this overview is to provide a comprehensive checklist of spiders of the region of South Asia, which includes eight countries, namely, Afghanistan, Bangladesh, Bhutan, India, Maldives, Nepal, Pakistan and Sri Lanka. Since the updated Indian checklist was published last year, only additions and changes that have occurred over the last year have been reported in the printed version of the paper. This printed overview includes checklists for seven countries of South Asia. The complete and 2006 updated checklist of Indian spiders is available on the web as a supplement to this printed version due to proibitive printing costs. Subsequent updates for all South Asian countries will be published on the web.

The spider descriptions in South Asia began in the late 18 th century with the first species described being Gasteracantha geminata by Fabricius (1798). However, before this period, there have been records of observation or collection of spiders by Dutch colonials in Sri Lanka. The earliest known formal illustration of a spider is of a mygalomorph by Madam Maria Sibylla Merian in the St. Petersburg Academy of Science published, approximately, in $1700 \mathrm{AD}$. It was only in 1804 that this species was formally described as Mygale fasciata by Latreille based on an illustration published in Albertas Seba's
Thesaurus, (Vol. 1) in 1734 (Smith, 2001). Most of the spiders described during the British period from South Asia were by foreigners based on the specimens deposited in different European Museums.

While the Indian checklist (Siliwal et al., 2005) is more accurate, the South Asian spider checklist is not critically scrutinized due to lack of complete literature, but it gives an overview of species found in various South Asian countries, gives the endemism of species and forms a basis for careful and participatory work by arachnologists in the region.

\section{AnAlysis}

The number of spider species reported so far from South Asia is 2299 belonging to 552 genera of 67 families (Checklist 9 on the web; Tables 1 \& 2 ). Cryptothelidae and Homalonychidae are the two monotypic families, represented by a single genus each. Out of the 552 genera, 49 (9\%) are monotypic, represented by single species and there are 65 genera $(12 \%)$ endemic to one or more South Asian countries (4 genera endemic to Afghanistan, one genus endemic to Bhutan, 20 genera endemic to India, four genera endemic to Nepal, one genus endemic to Pakistan, 22 genera endemic to Sri Lanka and 14 genera endemic to South Asia) (Table 2). Of the 2299 species, 1830 species (80\%) are endemic to South Asia (Table 2). Apart from this there are 15 subspecies reported from South Asia; nine subspecies are endemic to India, one subspecies each endemic to Afghanistan and Sri Lanka and four subspecies from Karakorum, whose endemicity is not known. Out of the 67 families of spiders in South Asia, seven families have not been reported from India. In the present checklist of South Asian spiders, the two families Zorocratidae and Zoropsidae are represented only in Sri Lanka, Cyrtaucheniidae in Afghanistan, Anapidae in Nepal, Cybaeidae in Karakorum and Himalaya, Dysderidae in Afghanistan and Karakorum, and Nesticidae in Afghanistan, Nepal and Sri Lanka.

\section{Afghanistan}

In Afghanistan, 113 species and one subspecies in 65 genera and 20 families have been reported; 95 species and four genera are monotypic and endemic to the country (Checklist 1; Table 2). Spider studies in Afghanistan began in 1846 with the description of the first taxon Eresus walckenaeri moerens by C.L. Koch. Until the middle of the 20th century only a few species were described. Major contribution to the spider studies in Afghanistan was done by Roewer (1955, 1960a,b, 1961, 1962) and Denis (1958) who described 64 and 23 species, respectively.

Manuscript 1509; (C) ZOO; Date of publication 24 January 2007 Received 14 January 2006; Finally accepted 09 January 2007 
Table 1. Representation of the spider families in South Asia and the World (Platnick, 2006)

\begin{tabular}{|c|c|c|c|c|c|c|}
\hline \multirow{2}{*}{\multicolumn{2}{|c|}{ Families }} & \multicolumn{3}{|c|}{ South Asia@ } & \multirow{2}{*}{$\begin{array}{l}\text { World * } \\
\quad \text { \# Sp. }\end{array}$} & \multirow{2}{*}{$\begin{array}{l}\text { Date of } \\
\text { updation } \$\end{array}$} \\
\hline & & \# Gen. & \# Sp. & \# Gen. & & \\
\hline 1. & Agelenidae & 3 & 16 & 39 & 503 & 25.ii.06 \\
\hline 2. & Amaurobiidae & 5 & 20 & 71 & 643 & 9.v.06 \\
\hline 3. & Anapidae & 1 & 2 & 34 & 144 & 3.ii.06 \\
\hline 4. & Anyphaenidae & 1 & 1 & 56 & 508 & 26.v.05 \\
\hline 5. & Araneidae & 34 & 178 & 166 & 2840 & 31.v.06 \\
\hline 6. & Atypidae & 1 & 1 & 3 & 40 & 2.iii.05 \\
\hline 7. & Barychelidae & 5 & 9 & 44 & 300 & 17.v.05 \\
\hline 8. & Cithaeronidae & 2 & 2 & 2 & 6 & 19.xi.02 \\
\hline 9. & Clubionidae & 4 & 33 & 15 & 538 & 5.v.06 \\
\hline 10. & Corinnidae & 14 & 57 & 76 & 925 & 3.v.06 \\
\hline 11. & Cryptothelidae \# & 1 & 2 & 1 & 10 & 21.xii.04 \\
\hline 12. & Ctenidae & 3 & 19 & 39 & 458 & 28.iii.06 \\
\hline 13. & Ctenizidae & 1 & 1 & 9 & 120 & $16 . x .05$ \\
\hline 14. & Cybaeidae & 2 & 2 & 12 & 153 & 3.v.06 \\
\hline 15. & Cyrtaucheniidae & 1 & 1 & 18 & 126 & 24.iii.05 \\
\hline 16. & Deinopidae & 1 & 1 & 4 & 57 & 18.xii.05 \\
\hline 17. & Desidae & 1 & 2 & 38 & 182 & 25.iv.06 \\
\hline 18. & Dictynidae & 10 & 15 & 48 & 562 & 1.v.06 \\
\hline 19. & Dipluridae & 2 & 5 & 24 & 175 & 24.iii.05 \\
\hline 20. & Dysderidae & 1 & 3 & 24 & 492 & 19.iii.06 \\
\hline 21. & Eresidae & 2 & 5 & 10 & 102 & 18.xii.05 \\
\hline 22. & Filistatidae & 3 & 12 & 16 & 109 & 18.xii.05 \\
\hline 23. & Gnaphosidae & 36 & 186 & 116 & 1975 & 19.iv.06 \\
\hline 24. & Hahniidae & 4 & 17 & 26 & 235 & 16.x.05 \\
\hline 25. & Hersiliidae & 5 & 12 & 11 & 145 & 15.i.06 \\
\hline 26. & Hexathelidae & 1 & 1 & 11 & 86 & 9.i.06 \\
\hline 27. & Homalonychidae \# & 1 & 1 & 1 & 3 & 21.xii.04 \\
\hline 28. & Idiopidae & 3 & 14 & 22 & 273 & 20.vi.06 \\
\hline 29. & Linyphiidae & 50 & 132 & 569 & 4320 & 9.v.06 \\
\hline 30. & Liocranidae & 3 & 11 & 29 & 160 & 14.ii.06 \\
\hline 31. & Lycosidae & 25 & 181 & 104 & 2304 & 11.vi.06 \\
\hline 32. & Mimetidae & 5 & 7 & 12 & 152 & 1.v.06 \\
\hline 33. & Miturgidae & 4 & 34 & 26 & 351 & 19.v.06 \\
\hline 34. & Mysmenidae & 3 & 4 & 22 & 91 & $16 . x .05$ \\
\hline 35. & Nemesiidae & 2 & 4 & 39 & 337 & 1.v.06 \\
\hline 36. & Nephilidae & 4 & 8 & 4 & 75 & $30 . v .06$ \\
\hline 37. & Nesticidae & 2 & 5 & 9 & 204 & 17.xii.05 \\
\hline 38. & Ochyroceratidae & 5 & 9 & 13 & 146 & 21.xii.03 \\
\hline 39. & Oecobiidae & 2 & 7 & 6 & 102 & 2.ii.06 \\
\hline 40. & Oonopidae & 10 & 33 & 68 & 472 & 4.iv.06 \\
\hline 41. & Oxyopidae & 4 & 74 & 9 & 419 & 23.v.06 \\
\hline 42. & Palpimanidae & 3 & 4 & 15 & 127 & 08.vi.04 \\
\hline 43. & Philodromidae & 8 & 51 & 29 & 517 & 5.v.06 \\
\hline 44. & Pholcidae & 9 & 20 & 80 & 959 & 20.vi.06 \\
\hline 45. & Pimoidae & 1 & 5 & 3 & 25 & 21.iii.06 \\
\hline 46. & Pisauridae & 10 & 21 & 52 & 328 & 16.x.05 \\
\hline 47. & Prodidomidae & 2 & 10 & 30 & 299 & 19.iv.06 \\
\hline 48. & Psechridae & 2 & 6 & 2 & 24 & 18.xii.05 \\
\hline 49. & Salticidae & 105 & 378 & 553 & 5025 & 23.v.06 \\
\hline 50. & Scytodidae & 1 & 12 & 5 & 169 & 9.v.06 \\
\hline 51. & Segestriidae & 2 & 4 & 3 & 106 & 16.x.05 \\
\hline 52. & Selenopidae & 1 & 6 & 4 & 189 & 17.xii.05 \\
\hline 53. & Sicariidae & 1 & 1 & 2 & 122 & 2.ii.06 \\
\hline 54. & Sparassidae & 14 & 141 & 82 & 1009 & 19.v.06 \\
\hline 55. & Stenochilidae & 1 & 3 & 2 & 12 & 26.iv.01 \\
\hline 56. & Tetrablemmidae & 7 & 16 & 29 & 126 & 28.iii.06 \\
\hline 57. & Tetragnathidae & 14 & 58 & 52 & 955 & 29.iii.06 \\
\hline 58. & Theraphosidae & 11 & 63 & 113 & 897 & 7.vi.06 \\
\hline 59. & Theridiidae & 26 & 88 & 87 & 2248 & 20.vi.06 \\
\hline 60. & Theridiosomatidae & 3 & 3 & 12 & 75 & 19.v.06 \\
\hline 61. & Thomisidae & 46 & 216 & 170 & 2026 & 12.vi.06 \\
\hline 62. & Titanoecidae & 2 & 2 & 5 & 46 & 18.xii.05 \\
\hline 63. & Trochanteriidae & 1 & 5 & 18 & 149 & 16.iii.06 \\
\hline 64. & Uloboridae & 5 & 25 & 18 & 262 & 3.v.06 \\
\hline 65. & Zodariidae & 8 & 30 & 72 & 828 & 3.v.06 \\
\hline 66. & Zorocratidae & 1 & 1 & 5 & 21 & 18.xii.05 \\
\hline 67. & Zoropsidae & 1 & 3 & 12 & 76 & 1.v.06 \\
\hline Tote & & 552 & 2298 & 3301 & 37463 & \\
\hline Wo & Id overall total ${ }^{\wedge}$ & & & 3642 & 39490 & 20.vi.06 \\
\hline
\end{tabular}

@ - Totals of South Asian spider species is not from Platnick (2006). It includes all new species described until date, but not yet included by Platnick.

* - A total of 111 families of spiders are listed in the World (Platnick, 2006) of which only those occurring in India are listed in the Table. The total at the end of the table is only a subset of the total spiders of the world. As of June 2006, Platnick lists 3,642 genera and 39,490 species worldwide, not including new species described in India since 2002.

$\$$ - Most recent date of updation of the families by Platnick (2006)

\# - Monotypic family

$\wedge$ - Sum total of all spiders of the world as listed in Platnick (2006). This total does not include the 39 new species described from India (Table 4).

\section{Bangladesh}

Fifty species in 24 genera and 13 families have been reported until date in Bangladesh of which 18 are endemic to the country (Checklist 2; Table 2). In spite of a large geographical area, there have been very few studies on spiders in this country. About 50\% (26 species) of the spiders reported from Bangladesh have been described from India. Recently, there has been a spurt in spider descriptions by Bangladesh arachnologists who have in the last seven years described 18 new species (Biswas, 1999; Biswas \& Begum, 1999; Biswas \& Raychaudhuri 1996a,b, 1998a,b, 2000, 2003a,b,c, 2004).

\section{Bhutan}

In Bhutan, 105 species of spiders in 51 genera and 12 families have been reported; a whopping $68 \%$ (71 species) are endemic to the country (Checklist 3; Table 2). The genus Dorjulopirata is endemic and monotypic to the country. Major contribution to spiders of Bhutan have been by foreigners like Zabka (1981, 1985, 1990) who described 30 species, Brignoli (1978) who described 15 species, and Ono (1978, 1979, 1980, 2000) who described 13 species. No recent works have come to our knowledge.

\section{India}

In India, 1447 species of spiders under 365 genera and 60 families have been recorded (Checklist 4 on the web). Since Siliwal et al. (2005) was published, there have been addition of one family Nephilidae, four genera Belisana Thorell, 1898, Eriophora Simon, 1864, Haplocosmia Schmidt \& von Wirth, 1996 and Martensopoda Jäger, 2006, and five new species Belisana dodabetta Huber, 2005, Belisana marusiki Huber, 2005, Clubiona maracandica Kroneberg, 1875, Martensopoda transversa Jäger, 2006 and Poecilotheria tigrinawesseli Smith, 2006. There are 19 genera endemic to the country and of which 14 are monotypic genera. Out of 1447 species, 1053 species are endemic to India (Table 2). The changes in the number of Indian endemics is either due to expansion in distribution range of the species or new knowledge in their occurrence outside of India. For example, Pardosa minuta Tikader \& Malhotra, 1976, Cheiracanthium sikkimense Majumder \& Tikader, 1991 and $C$. mysorense Majumder \& Tikader, 1991 were recently reported from Bangladesh (Platnick, 2006) and thus these Indian endemic species have now become South Asian endemics. Species like Lycosa chaperi Simon, 1885, Oxyopes wroughtoni Pocock, 1901, Oxyopes ryvesi Pocock, 1901, Myrmarachne ramunni Narayan, 1915 and Scytodes propinqua Stoliczka, 1869 were 
Table 2. List of spider genera represented in South Asia showing the number of species and endemicity.

\begin{tabular}{|c|c|c|c|c|c|c|c|c|c|c|}
\hline \multirow[t]{2}{*}{ Genus } & \multirow{2}{*}{$\begin{array}{l}\text { Total } \\
\text { spp. }\end{array}$} & \multirow[b]{2}{*}{ AF } & \multirow[b]{2}{*}{ BA } & \multirow[b]{2}{*}{$\mathrm{BH}$} & \multicolumn{3}{|c|}{ No. of Endemic Species } & \multirow[b]{2}{*}{ PA } & \multirow[b]{2}{*}{ SL } & \multirow[b]{2}{*}{ SA } \\
\hline & & & & & IN & MA & NE & & & \\
\hline I. Family Agelenidae C.L. Koch, 1837 & & & & & & & & & & \\
\hline 1. Agelena Walckenaer, 1805 & 8 & 0 & 0 & 0 & 6 & 0 & 2 & 0 & 0 & 8 \\
\hline 2. Tegenaria Latreille, 1804 & 7 & 0 & 0 & 1 & 3 & 0 & 1 & 0 & 1 & 6 \\
\hline 3. Tikaderia Lehtinen, 1967 \#@@ & 1 & 0 & 0 & 0 & 0 & 0 & 0 & 0 & 0 & 0 \\
\hline II. Family Amaurobiidae Thorell, 1870 & & & & & & & & & & \\
\hline 4. $\quad$ Amaurobius C.L. Koch, 1837 & 3 & 0 & 0 & 0 & 2 & 0 & 1 & 0 & 0 & 3 \\
\hline 5. Coelotes Blackwall, 1841 & 1 & 0 & 0 & 0 & 0 & 0 & 0 & 0 & 0 & 0 \\
\hline 6. Draconarius Ovtchinnikov, 1999 & 5 & 0 & 0 & 4 & 0 & 0 & 1 & 0 & 0 & 5 \\
\hline 7. Himalcoelotes Wang, 2002 & 10 & 0 & 0 & 1 & 0 & 0 & 8 & 0 & 0 & 9 \\
\hline 8. Tamgrinia Lehtinen, 1967 & 1 & 0 & 0 & 0 & 0 & 0 & 0 & 0 & 0 & 0 \\
\hline III. Family Anapidae & & & & & & & & & & \\
\hline 9. Metanapis Brignoli, 1981 & 2 & 0 & 0 & 0 & 0 & 0 & 2 & 0 & 0 & 2 \\
\hline IV. Family Anyphaenidae Bertkau, 1878 & & & & & & & & & & \\
\hline 10. Anyphaena Sundevall, 1833 & 1 & 0 & 0 & 0 & 1 & 0 & 0 & 0 & 0 & 1 \\
\hline V. Family Araneidae Simon, 1895 & & & & & & & & & & \\
\hline 11. Anepsion Strand, 1929 & 1 & 0 & 0 & 0 & 0 & 0 & 0 & 0 & 0 & 0 \\
\hline 12. Arachnura Vinson, 1863 & 2 & 0 & 0 & 0 & 1 & 0 & 0 & 0 & 0 & 1 \\
\hline 13. Araneus Clerck, 1757 & 25 & 0 & 0 & 0 & 10 & 0 & 0 & 2 & 1 & 15 \\
\hline 14. Araniella Chamberlin \& Ivie, 1942 & 1 & 0 & 0 & 0 & 0 & 0 & 0 & 0 & 0 & 0 \\
\hline 15. Argiope Audouin, 1826 & 10 & 0 & 0 & 0 & 0 & 0 & 0 & 0 & 1 & 2 \\
\hline 16. Cercidia Thorell, 1869 & 1 & 0 & 0 & 0 & 1 & 0 & 0 & 0 & 0 & 1 \\
\hline 17. Chorizopes O.P.-Cambridge, 1870 & 13 & 0 & 0 & 0 & 9 & 0 & 0 & 0 & 1 & 10 \\
\hline 18. Cyclosa Menge, 1866 & 25 & 0 & 4 & 0 & 6 & 0 & 0 & 3 & 0 & 14 \\
\hline 19. Cyrtarachne Thorell, 1868 & 11 & 0 & 0 & 0 & 7 & 0 & 0 & 0 & 0 & 8 \\
\hline 20. Cyrtophora Simon, 1864 & 10 & 0 & 2 & 0 & 3 & 0 & 0 & 0 & 0 & 5 \\
\hline 21. Eriophora Simon, 1864 & 1 & 0 & 0 & 0 & 0 & 0 & 0 & 0 & 0 & 0 \\
\hline 22. Eriovixia Archer, 1951 & 3 & 0 & 0 & 0 & 0 & 0 & 0 & 0 & 0 & 0 \\
\hline 23. Gasteracantha Sundevall, 1833 & 12 & 0 & 0 & 0 & 2 & 0 & 0 & 0 & 0 & 5 \\
\hline 24. Gea C.L. Koch, 1843 & 2 & 0 & 0 & 0 & 0 & 0 & 0 & 0 & 0 & 0 \\
\hline 25. Gibbaranea Archer, 1951 & 1 & 0 & 0 & 0 & 0 & 0 & 0 & 0 & 0 & 0 \\
\hline 26. Glyptogona Simon, 1884 & 1 & 0 & 0 & 0 & 0 & 0 & 0 & 0 & 1 & 1 \\
\hline 27. Homalopoltys Simon, $1895 @$ & 2 & 0 & 0 & 0 & 0 & 0 & 0 & 0 & 2 & 2 \\
\hline 28. Hypsosinga Ausserer, 1871 & 1 & 0 & 0 & 0 & 0 & 0 & 0 & 0 & 1 & 1 \\
\hline 29. Larinia Simon, 1874 & 7 & 0 & 0 & 0 & 5 & 0 & 0 & 0 & 0 & 5 \\
\hline 30. Lipocrea Thorell, 1878 & 1 & 0 & 0 & 0 & 0 & 0 & 0 & 0 & 0 & 0 \\
\hline 31. Macracantha Simon, $1864^{\#}$ & 1 & 0 & 0 & 0 & 0 & 0 & 0 & 0 & 0 & 0 \\
\hline 32. Mangora O. P.-Cambridge, 1889 & 1 & 0 & 0 & 0 & 0 & 0 & 0 & 0 & 1 & 1 \\
\hline 33. Neogea Levi, 1983 & 1 & 0 & 0 & 0 & 0 & 0 & 0 & 0 & 0 & 0 \\
\hline 34. Neoscona Simon, 1864 & 25 & 0 & 0 & 0 & 14 & 0 & 0 & 0 & 0 & 15 \\
\hline 35. Ordgarius Keyserling, 1886 & 3 & 0 & 0 & 0 & 1 & 0 & 0 & 0 & 0 & 1 \\
\hline 36. Parawixia F. O. P.-Cambridge, 1904 & 1 & 0 & 0 & 0 & 0 & 0 & 0 & 0 & 0 & 0 \\
\hline 37. Pasilobus Simon, 1895 & 1 & 0 & 0 & 0 & 1 & 0 & 0 & 0 & 0 & 1 \\
\hline 38. Poltys C.L. Koch, 1843 & 6 & 0 & 0 & 0 & 4 & 0 & 0 & 0 & 0 & 4 \\
\hline 39. Prasonica Simon, 1895 & 1 & 0 & 0 & 0 & 0 & 0 & 0 & 0 & 0 & 0 \\
\hline 40. Singa C.L. Koch, 1836 & 3 & 0 & 0 & 0 & 3 & 0 & 0 & 0 & 0 & 3 \\
\hline 41. Thelacantha Hasselt, $1882^{\#}$ & 1 & 0 & 0 & 0 & 0 & 0 & 0 & 0 & 0 & 0 \\
\hline 42. Ursa Simon, 1895 & 1 & 0 & 0 & 0 & 0 & 0 & 0 & 0 & 1 & 1 \\
\hline 43. Zilla C.L. Koch, 1834 & 1 & 0 & 0 & 0 & 1 & 0 & 0 & 0 & 0 & 1 \\
\hline 44. Zygiella F.O.P.-Cambridge, 1902 & 2 & 0 & 0 & 0 & 2 & 0 & 0 & 0 & 0 & 2 \\
\hline VI. Family Atypidae Thorell, 1870 & & & & & & & & & & \\
\hline 45. Atypus Latreille, 1804 & 1 & 0 & 0 & 0 & 1 & 0 & 0 & 0 & 0 & 1 \\
\hline VII. Family Barychelidae Simon, 1889 & & & & & & & & & & \\
\hline 46. Diplothele O. P.-Cambridge, 1890 ** & 2 & 0 & 0 & 0 & 0 & 0 & 0 & 0 & 1 & 2 \\
\hline 47. Plagiobothrus Karsch, $1891 \# @$ & 1 & 0 & 0 & 0 & 0 & 0 & 0 & 0 & 1 & 1 \\
\hline 48. Sason Simon, 1887 & 2 & 0 & 0 & 0 & 1 & 0 & 0 & 0 & 0 & 1 \\
\hline 49. Sasonichus Pocock, $1900^{\# *}$ & 1 & 0 & 0 & 0 & 1 & 0 & 0 & 0 & 0 & 1 \\
\hline 50. Sipalolasma Simon, 1892 & 3 & 0 & 0 & 0 & 1 & 0 & 0 & 0 & 2 & 3 \\
\hline VIII. Family Cithaeronidae Simon, 1893 & & & & & & & & & & \\
\hline 51. Cithaeron O.P.-Cambridge, 1872 & 1 & 0 & 0 & 0 & 1 & 0 & 0 & 0 & 0 & 1 \\
\hline 52. Inthaeron Platnick, $1991^{\# \star}$ & 1 & 0 & 0 & 0 & 1 & 0 & 0 & 0 & 0 & 1 \\
\hline IX. Family Clubionidae Wagner, 1887 & & & & & & & & & & \\
\hline 53. Clubiona Latreille, 1804 & 23 & 0 & 3 & 0 & 12 & 0 & 0 & 1 & 0 & 17 \\
\hline 54. Matidia Thorell, 1878 & 3 & 0 & 0 & 0 & 1 & 0 & 0 & 0 & 2 & 3 \\
\hline 55. Nusatidia Deeleman-Reinhold, 2001 & 1 & 0 & 0 & 0 & 0 & 0 & 0 & 0 & 1 & 1 \\
\hline 56. Simalio Simon, 1897 & 6 & 0 & 0 & 0 & 4 & 0 & 0 & 0 & 2 & 6 \\
\hline X. Family Corinnidae Karsch, 1880 & & & & & & & & & & \\
\hline 57. Aetius O.P.-Cambridge, 1896 & 1 & 0 & 0 & 0 & 0 & 0 & 0 & 0 & 0 & 1 \\
\hline
\end{tabular}




\begin{tabular}{|c|c|c|c|c|c|c|c|c|c|c|}
\hline \multirow[t]{2}{*}{ Genus } & \multirow{2}{*}{$\begin{array}{l}\text { Total } \\
\text { spp. }\end{array}$} & \multirow[b]{2}{*}{ AF } & \multirow[b]{2}{*}{ BA } & \multirow[b]{2}{*}{$\mathrm{BH}$} & \multicolumn{3}{|c|}{ No. of Endemic Species } & \multirow[b]{2}{*}{ PA } & \multirow[b]{2}{*}{ SL } & \multirow[b]{2}{*}{ SA } \\
\hline & & & & & IN & MA & NE & & & \\
\hline 58. Apochinomma Pavesi, 1881 & 2 & 0 & 0 & 0 & 1 & 0 & 0 & 0 & 0 & 1 \\
\hline 59. Castianeira Keyserling, 1879 & 10 & 1 & 0 & 0 & 7 & 0 & 0 & 0 & 0 & 9 \\
\hline 60. Coenoptychus Simon, $1885^{\# * *}$ & 1 & 0 & 0 & 0 & 0 & 0 & 0 & 0 & 0 & 1 \\
\hline 61. Copa Simon, 1885 & 2 & 0 & 0 & 0 & 0 & 0 & 0 & 0 & 2 & 2 \\
\hline 62. Corinna C. L. Koch, 1841 & 1 & 0 & 0 & 0 & 0 & 0 & 0 & 1 & 0 & 1 \\
\hline 63. Corinnomma Karsch, 1880 & 4 & 1 & 0 & 0 & 2 & 0 & 0 & 0 & 0 & 3 \\
\hline 64. Creugas Thorell, 1878 & 1 & 0 & 0 & 0 & 0 & 0 & 0 & 0 & 0 & 0 \\
\hline 65. Koppe Deeleman-Reinhold, 2001 & 1 & 0 & 0 & 0 & 0 & 0 & 0 & 0 & 1 & 1 \\
\hline 66. Oedignatha Thorell, 1881 & 26 & 0 & 0 & 0 & 15 & 0 & 0 & 0 & 10 & 25 \\
\hline 67. Orthobula Simon, 1897 & 1 & 0 & 0 & 0 & 0 & 0 & 0 & 0 & 0 & 0 \\
\hline 68. Sphecotypus O. P.-Cambridge, 1895 & 1 & 0 & 0 & 0 & 0 & 0 & 0 & 0 & 1 & 1 \\
\hline 69. Trachelas L. Koch, 1872 & 4 & 0 & 1 & 0 & 1 & 0 & 0 & 0 & 1 & 4 \\
\hline 70. Utivarachna Kishida, 1940 & 2 & 0 & 0 & 0 & 1 & 0 & 0 & 0 & 1 & 2 \\
\hline \multicolumn{11}{|l|}{ XI. Family Cryptothelidae L. Koch, $1872^{\# \# ~}$} \\
\hline 71. Cryptothele L. Koch, 1872 & 2 & 0 & 0 & 0 & 1 & 0 & 0 & 0 & 1 & 2 \\
\hline XII. Family Ctenidae Keyserling, 1877 & & & & & & & & & & \\
\hline 72. Acantheis Thorell, 1891 & 1 & 0 & 0 & 0 & 1 & 0 & 0 & 0 & 0 & 1 \\
\hline 73. Ctenus Walckenaer, 1805 & 16 & 0 & 0 & 0 & 13 & 0 & 0 & 0 & 3 & 16 \\
\hline 74. Diallomus Simon, 1897@ & 2 & 0 & 0 & 0 & 0 & 0 & 0 & 0 & 2 & 2 \\
\hline XIII. Family Ctenizidae Thorell, 1887 & & & & & & & & & & \\
\hline 75. Latouchia Pocock, 1901 & 1 & 0 & 0 & 0 & 1 & 0 & 0 & 0 & 0 & 1 \\
\hline XIV. Family Cybaeidae Banks, 1892 & & & & & & & & & & \\
\hline 76. Cedicus Simon, 1875 & 1 & 0 & 0 & 0 & 0 & 0 & 0 & 0 & 0 & 0 \\
\hline 77. Cybaeus L. Koch, 1868 & 1 & 0 & 0 & 0 & 0 & 0 & 0 & 0 & 0 & 0 \\
\hline XV. Family Cyrtaucheniidae Simon, 1892 & & & & & & & & & & \\
\hline 78. Anemesia Pocock, 1895 & 1 & 1 & 0 & 0 & 0 & 0 & 0 & 0 & 0 & 1 \\
\hline $\begin{array}{l}\text { XVI. Family Deinopidae C.L. Koch, } 1850 \\
\text { 79. Deinopis MacLeay, } 1839\end{array}$ & & & & & & & & & & \\
\hline 79. Deinopis MacLeay, 1839 & 1 & 0 & 0 & 0 & 1 & 0 & 0 & 0 & 0 & 1 \\
\hline $\begin{array}{l}\text { XVII. Family Desidae Pocock, } 1895 \\
80 . \quad \text { Desis Walckenaer, } 1837\end{array}$ & 2 & 0 & 0 & 0 & 2 & 0 & 0 & 0 & 0 & 2 \\
\hline XVIII. Family Dictynidae O.P.-Cambridge, & 1871 & & & & & & & & & \\
\hline 81. Ajmonia Caporiacco, 1934 & 1 & 0 & 0 & 0 & 0 & 0 & 0 & 0 & 0 & 0 \\
\hline 82. Anaxibia Thorell, 1898 & 2 & 0 & 0 & 0 & 1 & 0 & 0 & 0 & 0 & 2 \\
\hline 83. Argenna Thorell, 1870 & 1 & 0 & 0 & 0 & 0 & 0 & 0 & 0 & 0 & 0 \\
\hline 84. Atelolathys Simon, $1892 \# @$ & 1 & 0 & 0 & 0 & 0 & 0 & 0 & 0 & 1 & 1 \\
\hline 85. Dictyna Sundevall, 1833 & 3 & 0 & 0 & 0 & 1 & 0 & 0 & 0 & 0 & 2 \\
\hline 86. Dictynomorpha Spassky, 1939 & 3 & 0 & 0 & 0 & 2 & 0 & 0 & 0 & 1 & 3 \\
\hline 87. Lathys Simon, 1884 & 1 & 0 & 0 & 0 & 0 & 0 & 0 & 0 & 0 & 0 \\
\hline 88. Nigma Lehtinen, 1967 & 1 & 0 & 0 & 0 & 1 & 0 & 0 & 0 & 0 & 1 \\
\hline 89. Rhion O. P.-Cambridge, 1870 \# & 1 & 0 & 0 & 0 & 0 & 0 & 0 & 0 & 1 & 1 \\
\hline 90. Sudesna Lehtinen, 1967 & 1 & 0 & 0 & 0 & 1 & 0 & 0 & 0 & 0 & 1 \\
\hline XIX. Family Dipluridae Simon, 1889 & & & & & & & & & & \\
\hline 91. Indothele Coyle, 1995** & 4 & 0 & 0 & 0 & 3 & 0 & 0 & 0 & 1 & 4 \\
\hline 92. Ischnothele Ausserer, 1875 & 1 & 0 & 0 & 0 & 1 & 0 & 0 & 0 & 0 & 1 \\
\hline XX. Family Dysderidae C. L. Koch, 1837 & & & & & & & & & & \\
\hline 93. Dysdera Latreille, 1804 & 3 & 1 & 0 & 0 & 0 & 0 & 0 & 0 & 0 & 1 \\
\hline XXI. Family Eresidae C.L. Koch, 1851 & & & & & & & & & & \\
\hline 94. Eresus Walckenaer, 1805 & 1 & 0 & 0 & 0 & 0 & 0 & 0 & 0 & 0 & 0 \\
\hline 95. Stegodyphus Simon, 1873 & 4 & 0 & 0 & 0 & 1 & 0 & 0 & 0 & 0 & 2 \\
\hline XXII. Family Filistatidae Ausserer, 1867 & & & & & & & & & & \\
\hline 96. Filistata Latreille, 1810 & 5 & 1 & 0 & 0 & 3 & 0 & 0 & 0 & 0 & 4 \\
\hline 97. Pritha Lehtinen, 1967 & 5 & 1 & 0 & 0 & 4 & 0 & 0 & 0 & 0 & 5 \\
\hline 98. Sahastata Benoit, 1968 & 2 & 0 & 0 & 0 & 1 & 0 & 0 & 0 & 0 & 1 \\
\hline XXIII. Family Gnaphosidae Pocock, 1898 & & & & & & & & & & \\
\hline 99. Anagraphis Simon, 1893 & 1 & 1 & 0 & 0 & 0 & 0 & 0 & 0 & 0 & 1 \\
\hline 100. Apodrassodes Vellard, 1924 & 1 & 0 & 0 & 0 & 1 & 0 & 0 & 0 & 0 & 1 \\
\hline 101. Asiabadus Roewer, 1961 & 1 & 0 & 0 & 0 & 0 & 0 & 0 & 0 & 0 & 0 \\
\hline 102. Berlandina Dalmas, 1922 & 3 & 2 & 0 & 0 & 0 & 0 & 0 & 0 & 0 & 2 \\
\hline 103. Callilepis Westring, 1874 & 7 & 0 & 0 & 0 & 7 & 0 & 0 & 0 & 0 & 7 \\
\hline 104. Camillina Berland, 1919 & 1 & 0 & 0 & 0 & 1 & 0 & 0 & 0 & 0 & 1 \\
\hline 105. Drassodes Westring, 1851 & 35 & 8 & 0 & 0 & 19 & 0 & 1 & 0 & 0 & 30 \\
\hline 106. Drassyllus Chamberlin, 1922 & 4 & 0 & 0 & 0 & 4 & 0 & 0 & 0 & 0 & 4 \\
\hline 107. Echemus Simon, 1878 & 2 & 0 & 0 & 0 & 2 & 0 & 0 & 0 & 0 & 2 \\
\hline 108. Eilica Keyserling, 1891 & 4 & 0 & 0 & 0 & 4 & 0 & 0 & 0 & 0 & 4 \\
\hline 109. Gnaphosa Latreille, 1804 & 12 & 2 & 0 & 0 & 5 & 0 & 0 & 2 & 0 & 9 \\
\hline 110. Haplodrassus Chamberlin, 1922 & 8 & 0 & 0 & 0 & 7 & 0 & 0 & 0 & 0 & 7 \\
\hline 111. Herpyllus Hentz, 1832 & 6 & 3 & 0 & 0 & 2 & 0 & 0 & 0 & 0 & 5 \\
\hline
\end{tabular}




\begin{tabular}{|c|c|c|c|c|c|c|c|c|c|c|}
\hline \multirow{2}{*}{ Genus } & \multirow{2}{*}{$\begin{array}{l}\text { Total } \\
\text { spp. }\end{array}$} & \multirow[b]{2}{*}{$\mathbf{A F}$} & \multirow[b]{2}{*}{ BA } & \multirow[b]{2}{*}{$\mathrm{BH}$} & \multicolumn{3}{|c|}{ No. of Endemic Species } & \multirow[b]{2}{*}{ PA } & & \\
\hline & & & & & IN & MA & NE & & SL & SA \\
\hline 112. Kirmaka Roewer, $1961^{\# \$}$ & 1 & 1 & 0 & 0 & 0 & 0 & 0 & 0 & 0 & 1 \\
\hline 113. Ladissa Simon, 1907 & 2 & 0 & 0 & 0 & 2 & 0 & 0 & 0 & 0 & 2 \\
\hline 114. Megamyrmaekion Wider, 1834 & 3 & 0 & 0 & 0 & 3 & 0 & 0 & 0 & 0 & 3 \\
\hline 115. Micaria Westring, 1851 & 6 & 3 & 0 & 0 & 1 & 0 & 0 & 0 & 0 & 4 \\
\hline 116. Minosiella Dalmas, 1921 & 1 & 0 & 0 & 0 & 0 & 0 & 0 & 0 & 0 & 0 \\
\hline 117. Nodocion Chamberlin, 1922 & 2 & 0 & 0 & 0 & 2 & 0 & 0 & 0 & 0 & 2 \\
\hline 118. Nomisia Dalmas, 1921 & 2 & 1 & 0 & 0 & 1 & 0 & 0 & 0 & 0 & 2 \\
\hline 119. Odontodrassus Jézéquel, 1965 & 1 & 0 & 0 & 0 & 0 & 0 & 0 & 0 & 0 & 0 \\
\hline 120. Phaeocedus Simon, 1893 & 4 & 0 & 0 & 0 & 3 & 0 & 1 & 0 & 0 & 4 \\
\hline 121. Poecilochroa Westring, 1874 & 5 & 0 & 0 & 0 & 5 & 0 & 0 & 0 & 0 & 5 \\
\hline 121. Pterotricha Kulczyn'ski, 1903 & 1 & 0 & 0 & 0 & 1 & 0 & 0 & 0 & 0 & 1 \\
\hline 122. Pterotrichina Dalmas, 1921 & 1 & 0 & 0 & 0 & 0 & 0 & 0 & 0 & 0 & 0 \\
\hline 123. Scopoides Platnick, 1989 & 4 & 0 & 0 & 0 & 4 & 0 & 0 & 0 & 0 & 4 \\
\hline 124. Scotophaeus Simon, 1893 & 13 & 2 & 0 & 0 & 7 & 0 & 0 & 1 & 0 & 10 \\
\hline 125. Sergiolus Simon, 1891 & 5 & 0 & 0 & 0 & 5 & 0 & 0 & 0 & 0 & 5 \\
\hline 126. Setaphis Simon, 1893 & 2 & 0 & 0 & 0 & 0 & 0 & 0 & 0 & 0 & 0 \\
\hline 127. Sillemia Reimoser, $1935^{\# \wedge \wedge}$ & 1 & 0 & 0 & 0 & 0 & 0 & 0 & 0 & 0 & 0 \\
\hline 128. Siruasus Roewer, 1961 \#\$ & 1 & 1 & 0 & 0 & 0 & 0 & 0 & 0 & 0 & 1 \\
\hline 129. Sosticus Chamberlin, 1922 & 7 & 0 & 0 & 0 & 7 & 0 & 0 & 0 & 0 & 7 \\
\hline 130. Synaphosus Platnick \& Shadab, 1980 & 1 & 0 & 0 & 0 & 0 & 0 & 0 & 0 & 0 & 0 \\
\hline 131. Talanites Simon, 1893 & 1 & 0 & 0 & 0 & 0 & 0 & 0 & 0 & 0 & 1 \\
\hline 132. Trachyzelotes Lohmander, 1944 & 1 & 0 & 0 & 0 & 0 & 0 & 0 & 0 & 0 & 0 \\
\hline 133. Urozelotes Mello-Leitão, 1938 & 1 & 0 & 0 & 0 & 0 & 0 & 0 & 0 & 0 & 0 \\
\hline 134. Zelotes Gistel, 1848 & 36 & 7 & 0 & 0 & 23 & 0 & 0 & 4 & 0 & 35 \\
\hline XXIV. Family Hahniidae Bertkau, 1878 & & & & & & & & & & \\
\hline 135. Alistra Thorell, 1894 & 3 & 0 & 0 & 0 & 0 & 0 & 0 & 0 & 3 & 3 \\
\hline 136. Hahnia C.L. Koch, 1841 & 10 & 1 & 0 & 5 & 1 & 0 & 1 & 0 & 2 & 10 \\
\hline 137. Neoantistea Gertsch, 1934 & 3 & 0 & 0 & 0 & 2 & 0 & 1 & 0 & 0 & 3 \\
\hline 138. Scotospilus Simon, 1886 & 1 & 0 & 0 & 0 & 1 & 0 & 0 & 0 & 0 & 1 \\
\hline XXV. Family Hersiliidae Thorell, 1870 & & & & & & & & & & \\
\hline 139. Hersilia Audouin, 1826 & 5 & 0 & 0 & 0 & 0 & 0 & 2 & 0 & 0 & 3 \\
\hline 140. Hersiliola Thorell, 1870 & 1 & 0 & 0 & 0 & 0 & 0 & 0 & 0 & 0 & 0 \\
\hline 141. Murricia Simon, 1882 & 2 & 0 & 0 & 0 & 1 & 0 & 0 & 0 & 1 & 2 \\
\hline 142. Neotama Baehr \& Baehr, 1993 & 3 & 0 & 0 & 0 & 2 & 0 & 0 & 0 & 1 & 3 \\
\hline 143. Promurricia Baehr \& Baehr, 1993 \#@ & 1 & 0 & 0 & 0 & 0 & 0 & 0 & 0 & 1 & 1 \\
\hline XXVI. Family Hexathelidae Simon, 1892 & & & & & & & & & & \\
\hline 144. Macrothele Ausserer, 1871 & 1 & 0 & 0 & 0 & 1 & 0 & 0 & 0 & 0 & 1 \\
\hline XXVII. Family Homalonychidae Simon, 1893 & & & & & & & & & & \\
\hline 145. Homalonychus Marx, 1891 & 1 & 0 & 0 & 0 & 1 & 0 & 0 & 0 & 0 & 1 \\
\hline XXVIII. Family Idiopidae Simon, 1892 & & & & & & & & & & \\
\hline 146. Heligmomerus Simon, 1892 & 2 & 0 & 0 & 0 & 1 & 0 & 0 & 0 & 1 & 2 \\
\hline 147. Idiops Perty, 1833 & 9 & 0 & 0 & 0 & 8 & 0 & 0 & 1 & 0 & 9 \\
\hline 148. Scalidognathus Karsch, 1891 & 3 & 0 & 0 & 0 & 1 & 0 & 0 & 0 & 2 & 3 \\
\hline XXIX. Family Linyphiidae Blackwall, 1859 & & & & & & & & & & \\
\hline 149. Agyneta Hull, 1911 & 4 & 0 & 0 & 0 & 0 & 0 & 4 & 0 & 0 & 4 \\
\hline 150. Alioranus Simon, 1926 & 2 & 0 & 0 & 0 & 0 & 0 & 0 & 0 & 0 & 0 \\
\hline 151. Arachosinella Denis, 1958 & 1 & 0 & 0 & 0 & 0 & 0 & 0 & 0 & 0 & 0 \\
\hline 152. Araeoncus Simon, 1884 & 1 & 0 & 0 & 0 & 0 & 0 & 0 & 0 & 0 & 0 \\
\hline 153. Asthenargus Simon \& Fage, 1922 & 1 & 0 & 0 & 0 & 0 & 0 & 1 & 0 & 0 & 1 \\
\hline 154. Atypena Simon, 1894 & 2 & 0 & 0 & 0 & 0 & 0 & 0 & 0 & 2 & 2 \\
\hline 155. Bathyphantes Menge, 1866 & 3 & 0 & 0 & 0 & 0 & 0 & 0 & 0 & 0 & 0 \\
\hline 156. Callitrichia Fage, 1936 & 1 & 0 & 0 & 0 & 0 & 0 & 0 & 0 & 0 & 0 \\
\hline 157. Caviphantes Oi, 1960 & 1 & 0 & 0 & 0 & 0 & 0 & 0 & 0 & 0 & 0 \\
\hline 158. Ceratinopsis Emerton, 1882 & 1 & 0 & 0 & 0 & 0 & 0 & 0 & 0 & 1 & 1 \\
\hline 159. Collinsia O. P.-Cambridge, 1913 & 1 & 0 & 0 & 0 & 1 & 0 & 0 & 0 & 0 & 1 \\
\hline 160. Cresmatoneta Simon, 1929 & 1 & 0 & 0 & 0 & 1 & 0 & 0 & 0 & 0 & 1 \\
\hline 161. Emenista Simon, 1894 & 1 & 0 & 0 & 0 & 1 & 0 & 0 & 0 & 0 & 1 \\
\hline 162. Erigone Audouin, 1826 & 4 & 0 & 0 & 0 & 1 & 0 & 1 & 0 & 0 & 2 \\
\hline 163. Gongylidiellum Simon, 1884 & 5 & 0 & 0 & 0 & 1 & 0 & 2 & 0 & 0 & 3 \\
\hline 164. Gongylidium Menge, 1868 & 1 & 0 & 0 & 0 & 0 & 0 & 0 & 0 & 0 & 0 \\
\hline 165. Gorbothorax Tanasevitch, $1998 \%$ & 5 & 0 & 0 & 0 & 0 & 0 & 5 & 0 & 0 & 5 \\
\hline 166. Helsdingenia Saaristo \& & 1 & 0 & 0 & 0 & 0 & 0 & 0 & 0 & 0 & 1 \\
\hline Tanasevitch, 2003 & & & & & & & & & & \\
\hline 167. Heterolinyphia Wunderlich, 1973 ** & 2 & 0 & 0 & 1 & 0 & 0 & 0 & 0 & 0 & 2 \\
\hline 168. Hilaira Simon, 1884 & 1 & 0 & 0 & 0 & 0 & 0 & 1 & 0 & 0 & 1 \\
\hline 169. Himalaphantes Tanasevitch, 1992 & 3 & 0 & 0 & 0 & 0 & 0 & 2 & 0 & 0 & 3 \\
\hline 170. Hubertella Platnick, $1989 \%$ & 2 & 0 & 0 & 0 & 0 & 0 & 2 & 0 & 0 & 2 \\
\hline 171. Indophantes Saaristo \& Tanasevitch, 2003 & 3 & 0 & 0 & 0 & 2 & 0 & 0 & 0 & 0 & 3 \\
\hline 172. Labulla Simon, 1884 & 1 & 0 & 0 & 0 & 1 & 0 & 0 & 0 & 0 & 1 \\
\hline 173. Labullinyphia van Helsdingen, 1985\#@ & 1 & 0 & 0 & 0 & 0 & 0 & 0 & 0 & 1 & 1 \\
\hline 174. Lepthyphantes Menge, 1866 & 27 & 1 & 0 & 0 & 3 & 0 & 15 & 0 & 0 & 19 \\
\hline
\end{tabular}




\begin{tabular}{|c|c|c|c|c|c|c|c|c|c|c|}
\hline \multirow[t]{2}{*}{ Genus } & \multirow{2}{*}{$\begin{array}{l}\text { Total } \\
\text { spp. }\end{array}$} & \multirow[b]{2}{*}{ AF } & \multirow[b]{2}{*}{ BA } & \multirow[b]{2}{*}{$\mathrm{BH}$} & \multicolumn{3}{|c|}{ No. of Endemic Species } & \multirow[b]{2}{*}{ PA } & \multirow[b]{2}{*}{ SL } & \multirow[b]{2}{*}{ SA } \\
\hline & & & & & IN & MA & NE & & & \\
\hline 175. Linyphia Latreille, 1804 & 6 & 0 & 0 & 0 & 5 & 0 & 1 & 0 & 0 & 6 \\
\hline 176. Martensinus Wunderlich, $1973 \%$ & 2 & 0 & 0 & 0 & 0 & 0 & 2 & 0 & 0 & 2 \\
\hline 177. Meioneta Hull, 1920 & 1 & 0 & 0 & 0 & 0 & 0 & 1 & 0 & 0 & 1 \\
\hline $\begin{array}{l}\text { 178. Microbathyphantes van Helsdingen, } \\
1985\end{array}$ & 1 & 0 & 0 & 0 & 0 & 0 & 0 & 0 & 0 & 0 \\
\hline 179. Microctenonyx Dahl, 1886 & 1 & 0 & 0 & 0 & 0 & 0 & 0 & 0 & 0 & 0 \\
\hline 180. Minicia Thorell, 1875 & 1 & 0 & 0 & 0 & 1 & 0 & 0 & 0 & 0 & 1 \\
\hline $\begin{array}{l}\text { 181. Mughiphantes Saaristo \& } \\
\text { Tanasevitch, } 1999\end{array}$ & 1 & 1 & 0 & 0 & 0 & 0 & 0 & 0 & 0 & 1 \\
\hline 182. Nematogmus Simon, 1884 & 1 & 0 & 0 & 0 & 0 & 0 & 0 & 0 & 0 & 0 \\
\hline 183. Neriene Blackwall, 1833 & 2 & 0 & 0 & 0 & 0 & 0 & 0 & 0 & 1 & 1 \\
\hline 184. Nesioneta Millidge, 1991 & 1 & 0 & 0 & 0 & 0 & 0 & 0 & 0 & 0 & 0 \\
\hline 185. Obrimona Strand, 1934 \#@ & 1 & 0 & 0 & 0 & 0 & 0 & 0 & 0 & 1 & 1 \\
\hline 186. Oedothorax Bertkau, 1883 & 22 & 0 & 0 & 0 & 2 & 0 & 20 & 0 & 0 & 22 \\
\hline 187. Oia Wunderlich, 1973 & 1 & 0 & 0 & 0 & 0 & 0 & 1 & 0 & 0 & 1 \\
\hline 188. Paragongylidiellum Wunderlich, $1973^{\# \%}$ & 1 & 0 & 0 & 0 & 0 & 0 & 1 & 0 & 0 & 1 \\
\hline $\begin{array}{l}\text { 189. Piniphantes Saaristo \& } \\
\text { Tanasevitch, } 1996\end{array}$ & 1 & 0 & 0 & 0 & 0 & 0 & 1 & 0 & 0 & 1 \\
\hline 190. Pocadicnemis Simon, 1884 & 1 & 0 & 0 & 0 & 0 & 0 & 0 & 0 & 0 & 0 \\
\hline 191. Porrhomma Simon, 1884 & 1 & 0 & 0 & 0 & 0 & 0 & 1 & 0 & 0 & 1 \\
\hline 192. Saloca Simon, 1926 & 2 & 0 & 0 & 0 & 0 & 0 & 2 & 0 & 0 & 2 \\
\hline $\begin{array}{l}\text { 193. Tenuiphantes Saaristo \& } \\
\text { Tanasevitch, } 1996\end{array}$ & 1 & 0 & 0 & 0 & 0 & 0 & 1 & 0 & 0 & 1 \\
\hline 194. Tiso Simon, 1884 & 1 & 0 & 0 & 0 & 0 & 0 & 0 & 0 & 0 & 0 \\
\hline 195. Trematocephalus Dahl, 1886 & 2 & 0 & 0 & 0 & 0 & 0 & 0 & 0 & 2 & 2 \\
\hline 196. Troxochrota Kulczyn'ski, 1894 & 1 & 0 & 0 & 0 & 1 & 0 & 0 & 0 & 0 & 1 \\
\hline 197. Typhistes Simon, 1894 & 2 & 0 & 0 & 0 & 0 & 0 & 0 & 0 & 2 & 2 \\
\hline 198. Walckenaeria Blackwall, 1833 & 2 & 0 & 0 & 0 & 0 & 0 & 2 & 0 & 0 & 2 \\
\hline $\begin{array}{l}\text { 199. Argistes Simon, } 1897 \\
\text { 200. Paratus Simon, } 1898 \# @\end{array}$ & $\begin{array}{l}2 \\
1\end{array}$ & $\begin{array}{l}0 \\
0\end{array}$ & $\begin{array}{l}0 \\
0\end{array}$ & $\begin{array}{l}0 \\
0\end{array}$ & $\begin{array}{l}0 \\
0\end{array}$ & $\begin{array}{l}0 \\
0\end{array}$ & $\begin{array}{l}0 \\
0\end{array}$ & $\begin{array}{l}0 \\
0\end{array}$ & $\begin{array}{l}2 \\
1\end{array}$ & $\begin{array}{l}2 \\
1\end{array}$ \\
\hline 201. Sphingius Thorell, 1890 & 8 & 0 & 0 & 0 & 6 & 0 & 0 & 0 & 1 & 8 \\
\hline XXXI. Family Lycosidae Sundevall, 1833 & & & & & & & & & & \\
\hline 202. Acantholycosa Dahl, 1908 & 1 & 0 & 0 & 0 & 0 & 0 & 0 & 0 & 0 & 0 \\
\hline 203. Agalenocosa Mello-Leitão, 1944 & 1 & 0 & 0 & 0 & 1 & 0 & 0 & 0 & 0 & 1 \\
\hline 204. Allocosa Banks, 1900 & 3 & 3 & 0 & 0 & 0 & 0 & 0 & 0 & 0 & 3 \\
\hline 205. Alopecosa Simon, 1885 & 2 & 2 & 0 & 0 & 0 & 0 & 0 & 0 & 0 & 2 \\
\hline 206. Anomalosa Roewer, 1960 & 1 & 0 & 0 & 0 & 0 & 0 & 0 & 1 & 0 & 1 \\
\hline 207. Arctosa C.L. Koch, 1847 & 12 & 3 & 0 & 0 & 4 & 0 & 1 & 0 & 0 & 9 \\
\hline 208. Crocodilosa Caporiacco, 1947 & 2 & 0 & 0 & 0 & 2 & 0 & 0 & 0 & 0 & 2 \\
\hline 209. Dorjulopirata Buchar, $1997^{\# \wedge}$ & 1 & 0 & 0 & 1 & 0 & 0 & 0 & 0 & 0 & 1 \\
\hline 210. Evippa Simon, 1882 & 10 & 1 & 0 & 0 & 8 & 0 & 0 & 0 & 0 & 9 \\
\hline 211. Evippomma Roewer, 1959 & 2 & 0 & 0 & 0 & 1 & 0 & 0 & 0 & 0 & 1 \\
\hline 212. Geolycosa Montgomery, 1904 & 3 & 1 & 0 & 0 & 1 & 0 & 0 & 0 & 0 & 2 \\
\hline 213. Hippasa Simon, 1885 & 20 & 1 & 0 & 1 & 10 & 0 & 0 & 0 & 0 & 12 \\
\hline 214. Hogna Simon, 1885 & 5 & 1 & 0 & 0 & 0 & 0 & 0 & 0 & 1 & 2 \\
\hline 215. Lycosa Latreille, 1804 & 33 & 1 & 0 & 0 & 23 & 0 & 0 & 0 & 1 & 29 \\
\hline 216. Margonia Hippa \& Lehtinen, $1983^{\# \star}$ & 1 & 0 & 0 & 0 & 1 & 0 & 0 & 0 & 0 & 1 \\
\hline 217. Megarctosa Caporiacco, 19481 & 1 & 1 & 0 & 0 & 0 & 0 & 0 & 0 & 0 & 1 \\
\hline 218. Ocyale Audouin, 1826 & 4 & 0 & 0 & 0 & 1 & 0 & 0 & 1 & 1 & 3 \\
\hline 219. Pardosa C.L. Koch, 1847 & 64 & 10 & 1 & 0 & 25 & 0 & 2 & 1 & 1 & 47 \\
\hline 220. Passiena Thorell, 1890 & 1 & 0 & 0 & 0 & 0 & 0 & 0 & 0 & 0 & 0 \\
\hline 221. Shapna Hippa \& Lehtinen, 1983 \#* & 1 & 0 & 0 & 0 & 1 & 0 & 0 & 0 & 0 & 1 \\
\hline 222. Schizocosa Chamberlin, 1904 & 3 & 2 & 0 & 0 & 0 & 0 & 0 & 0 & 0 & 2 \\
\hline 223. Trochosa C.L. Koch, 1847 & 5 & 0 & 0 & 1 & 3 & 0 & 1 & 0 & 0 & 5 \\
\hline 224. Trochosula Roewer, 1960 & 1 & 1 & 0 & 0 & 0 & 0 & 0 & 0 & 0 & 1 \\
\hline 225. Wadicosa Zyuzin, 1985 & 1 & 0 & 0 & 0 & 0 & 0 & 0 & 0 & 0 & 1 \\
\hline 226. Zoica Simon, 1898 & 3 & 0 & 0 & 1 & 0 & 0 & 0 & 0 & 0 & 2 \\
\hline XXXII. Family Mimetidae Simon, 1881 & & & & & & & & & & \\
\hline 227. Ero C. L. Koch, 1836 & 1 & 0 & 0 & 1 & 0 & 0 & 0 & 0 & 0 & 1 \\
\hline 228. Gelanor Thorell, 1869 & 1 & 0 & 0 & 0 & 0 & 0 & 0 & 1 & 0 & 1 \\
\hline 229. Melaenosia Simon, 1906 \#* & 1 & 0 & 0 & 0 & 1 & 0 & 0 & 0 & 0 & 1 \\
\hline 230. Mimetus Hentz, 1832 & 3 & 0 & 0 & 0 & 2 & 0 & 0 & 0 & 1 & 3 \\
\hline 231. Phobetinus Simon, 1895 & 1 & 0 & 0 & 0 & 0 & 0 & 0 & 0 & 1 & 1 \\
\hline XXXIII. Family Miturgidae Simon, 1885 & & & & & & & & & & \\
\hline 232. Cheiracanthium C.L. Koch, 1839 & 29 & 0 & 1 & 0 & 17 & 0 & 0 & 0 & 2 & 23 \\
\hline 233. Eutichurus Simon, 1897 & 2 & 0 & 0 & 0 & 2 & 0 & 0 & 0 & 0 & 2 \\
\hline 234. Strotarchus Simon, 1888 & 2 & 0 & 0 & 0 & 0 & 0 & 0 & 2 & 0 & 2 \\
\hline 235. Systaria Simon, 1897 & 1 & 0 & 0 & 0 & 1 & 0 & 0 & 0 & 0 & 1 \\
\hline XXXIV. Family Mysmenidae Petrunkevitch, & 1928 & & & & & & & & & \\
\hline 236. Iardinis Simon, $1899^{* *}$ & 2 & 0 & 0 & 0 & 1 & 0 & 1 & 0 & 0 & 2 \\
\hline 237. Mysmenella Brignoli, 1980 & 1 & 0 & 0 & 0 & 0 & 0 & 0 & 0 & 1 & 1 \\
\hline
\end{tabular}




\begin{tabular}{|c|c|c|c|c|c|c|c|c|c|c|}
\hline \multirow[t]{2}{*}{ Genus } & \multirow{2}{*}{$\begin{array}{l}\text { Total } \\
\text { spp. }\end{array}$} & \multirow[b]{2}{*}{ AF } & \multirow[b]{2}{*}{ BA } & \multirow[b]{2}{*}{$\mathrm{BH}$} & \multicolumn{3}{|c|}{ No. of Endemic Species } & \multirow[b]{2}{*}{ PA } & \multirow[b]{2}{*}{ SL } & \multirow[b]{2}{*}{ SA } \\
\hline & & & & & IN & MA & $\mathrm{NE}$ & & & \\
\hline 238. Phricotelus Simon, 1895 \#@ & 1 & 0 & 0 & 0 & 0 & 0 & 0 & 0 & 1 & 1 \\
\hline $\begin{array}{l}\text { XXXV. Family Nemesiidae Simon, } 1892 \\
\text { 239. Damarchus Thorell, } 1891 \\
\text { 240. Raveniola Zonstein, } 1987\end{array}$ & $\begin{array}{l}3 \\
1\end{array}$ & $\begin{array}{l}0 \\
0\end{array}$ & $\begin{array}{l}0 \\
0\end{array}$ & $\begin{array}{l}0 \\
0\end{array}$ & $\begin{array}{l}3 \\
0\end{array}$ & $\begin{array}{l}0 \\
0\end{array}$ & $\begin{array}{l}0 \\
0\end{array}$ & $\begin{array}{l}0 \\
0\end{array}$ & $\begin{array}{l}0 \\
0\end{array}$ & $\begin{array}{l}3 \\
0\end{array}$ \\
\hline $\begin{array}{l}\text { XXXVI. Family Nephilidae Simon, } 1894 \\
\text { 241. Clitaetra Simon, } 1889 \\
\text { 242. Herennia Thorell, } 1877 \\
\text { 243. Nephila Leach, } 1815 \\
\text { 244. Nephilengys L. Koch, } 1872\end{array}$ & $\begin{array}{l}1 \\
1 \\
5 \\
1\end{array}$ & $\begin{array}{l}0 \\
0 \\
0 \\
0\end{array}$ & $\begin{array}{l}0 \\
0 \\
0 \\
0\end{array}$ & $\begin{array}{l}0 \\
0 \\
0 \\
0\end{array}$ & $\begin{array}{l}0 \\
0 \\
1 \\
0\end{array}$ & $\begin{array}{l}0 \\
0 \\
0 \\
0\end{array}$ & $\begin{array}{l}0 \\
0 \\
0 \\
0\end{array}$ & $\begin{array}{l}0 \\
0 \\
1 \\
0\end{array}$ & $\begin{array}{l}1 \\
0 \\
0 \\
0\end{array}$ & $\begin{array}{l}1 \\
0 \\
2 \\
0\end{array}$ \\
\hline $\begin{array}{l}\text { XXXVII. Family Nesticidae Simon, } 1894 \\
\text { 245. Nesticella Lehtinen \& } \\
\text { Saaristo, } 1980 \\
\text { 246. Nesticus Thorell, } 1869\end{array}$ & 2 & 0 & 0 & 0 & 0 & 0 & 1 & 0 & 1 & 2 \\
\hline $\begin{array}{l}\text { XXXVIII. Family Ochyroceratidae Fage, } 19 \\
\text { 247. Althepus Thorell, } 1898 \\
\text { 248. Leclercera Deeleman-Reinhold, } 1995 \\
\text { 249. Merizocera Fage, } 1912 \\
\text { 250. Psiloderces Simon, } 1892 \\
\text { 251. Speocera Berland, } 1914\end{array}$ & $\begin{array}{r}12 \\
1 \\
1 \\
4 \\
2 \\
1\end{array}$ & $\begin{array}{l}0 \\
0 \\
0 \\
0 \\
0\end{array}$ & $\begin{array}{l}0 \\
0 \\
0 \\
0 \\
0\end{array}$ & $\begin{array}{l}0 \\
0 \\
0 \\
0 \\
0\end{array}$ & $\begin{array}{l}1 \\
0 \\
0 \\
0 \\
0\end{array}$ & $\begin{array}{l}0 \\
0 \\
0 \\
0 \\
0\end{array}$ & $\begin{array}{l}0 \\
1 \\
0 \\
1 \\
0\end{array}$ & $\begin{array}{l}0 \\
0 \\
0 \\
0 \\
0\end{array}$ & $\begin{array}{l}0 \\
0 \\
4 \\
1 \\
1\end{array}$ & $\begin{array}{l}1 \\
1 \\
4 \\
2 \\
1\end{array}$ \\
\hline $\begin{array}{l}\text { XXXIX. Family Oecobiidae Blackwall, } 1862 \\
\text { 252. Oecobius Lucas, } 1846 \\
\text { 253. Uroctea Dufour, } 1820\end{array}$ & $\begin{array}{l}3 \\
4\end{array}$ & $\begin{array}{l}0 \\
1\end{array}$ & $\begin{array}{l}0 \\
0\end{array}$ & $\begin{array}{l}0 \\
0\end{array}$ & $\begin{array}{l}1 \\
2\end{array}$ & $\begin{array}{l}0 \\
0\end{array}$ & $\begin{array}{l}0 \\
0\end{array}$ & $\begin{array}{l}0 \\
1\end{array}$ & $\begin{array}{l}0 \\
0\end{array}$ & $\begin{array}{l}1 \\
4\end{array}$ \\
\hline $\begin{array}{l}\text { XL. Family Oonopidae Simon, } 1890 \\
\text { 254. Aprusia Simon, } 1893^{\#} \\
\text { 255. Camptoscaphiella Caporiacco, } 1934 \\
\text { 256. Dysderoides Fage, } 1946 \\
\text { 257. Epectris Simon, } 1893 \\
\text { 258. Gamasomorpha Karsch, } 1881 \\
\text { 259. Ischnothyreus Simon, } 1893 \\
\text { 260. Opopaea Simon, } 1891 \\
\text { 261. Orchestina Simon, } 1882 \\
\text { 262. Triaeris Simon, } 1891 \\
\text { 263. Xestaspis Simon, } 1884\end{array}$ & $\begin{array}{l}1 \\
3 \\
1 \\
2 \\
6 \\
5 \\
2 \\
5 \\
7 \\
1\end{array}$ & $\begin{array}{l}0 \\
0 \\
0 \\
0 \\
1 \\
0 \\
0 \\
0 \\
0 \\
0\end{array}$ & $\begin{array}{l}0 \\
0 \\
0 \\
0 \\
0 \\
0 \\
0 \\
0 \\
0 \\
0\end{array}$ & $\begin{array}{l}0 \\
1 \\
0 \\
1 \\
0 \\
0 \\
1 \\
1 \\
0 \\
0\end{array}$ & $\begin{array}{l}0 \\
0 \\
1 \\
0 \\
1 \\
1 \\
0 \\
0 \\
7 \\
0\end{array}$ & $\begin{array}{l}0 \\
0 \\
0 \\
0 \\
0 \\
0 \\
0 \\
0 \\
0 \\
0\end{array}$ & $\begin{array}{l}0 \\
2 \\
0 \\
0 \\
0 \\
0 \\
0 \\
0 \\
0 \\
0\end{array}$ & $\begin{array}{l}0 \\
0 \\
0 \\
0 \\
0 \\
0 \\
0 \\
0 \\
0 \\
0\end{array}$ & $\begin{array}{l}1 \\
0 \\
0 \\
1 \\
2 \\
3 \\
1 \\
3 \\
0 \\
1\end{array}$ & $\begin{array}{l}1 \\
3 \\
1 \\
2 \\
6 \\
5 \\
2 \\
4 \\
7 \\
1\end{array}$ \\
\hline $\begin{array}{l}\text { XLI. Family Oxyopidae Thorell, } 1870 \\
\text { 264. Hamataliwa Keyserling, } 1887 \\
\text { 265. Oxyopes Latreille, } 1804 \\
\text { 266. Peucetia Thorell, } 1869 \\
\text { 267. Tapponia Simon, } 1885\end{array}$ & $\begin{array}{l}1 \\
55 \\
17 \\
1\end{array}$ & $\begin{array}{l}0 \\
0 \\
0 \\
0\end{array}$ & $\begin{array}{l}0 \\
0 \\
0 \\
0\end{array}$ & $\begin{array}{l}0 \\
0 \\
0 \\
0\end{array}$ & $\begin{array}{l}0 \\
34 \\
14 \\
1\end{array}$ & $\begin{array}{l}0 \\
0 \\
0 \\
0\end{array}$ & $\begin{array}{l}0 \\
0 \\
0 \\
0\end{array}$ & $\begin{array}{l}0 \\
4 \\
0 \\
0\end{array}$ & $\begin{array}{l}0 \\
4 \\
0 \\
0\end{array}$ & $\begin{array}{l}0 \\
45 \\
14 \\
1\end{array}$ \\
\hline $\begin{array}{l}\text { XLII. Family Palpimanidae Thorell, } 1870 \\
\text { 268. Palpimanus Dufour, } 1820 \\
\text { 269. Sarascelis Simon, } 1897 \\
\text { 270. Steriphopus Simon, } 1887\end{array}$ & $\begin{array}{l}2 \\
1 \\
1\end{array}$ & $\begin{array}{l}0 \\
0 \\
0\end{array}$ & $\begin{array}{l}0 \\
0 \\
0\end{array}$ & $\begin{array}{l}0 \\
0 \\
0\end{array}$ & $\begin{array}{l}1 \\
0 \\
0\end{array}$ & $\begin{array}{l}0 \\
0 \\
0\end{array}$ & $\begin{array}{l}0 \\
0 \\
0\end{array}$ & $\begin{array}{l}0 \\
0 \\
0\end{array}$ & $\begin{array}{l}0 \\
0 \\
1\end{array}$ & $\begin{array}{l}1 \\
0 \\
1\end{array}$ \\
\hline $\begin{array}{l}\text { XLIII. Family Philodromidae Thorell, } 1870 \\
\text { 271. Apollophanes O. P.-Cambridge, } 1898 \\
\text { 272. Ebo Keyserling, } 1884 \\
\text { 273. Gephyrota Strand, } 1932 \\
\text { 274. Philodromus Walckenaer, } 1826 \\
\text { 275. Psellonus Simon, } 1897^{\star \#} \\
\text { 276. Thanatus C.L. Koch, } 1837 \\
\text { 277. Tibellus Simon, } 1875 \\
\text { 278. Vacchellia Caporiacco, } 1935^{\# \wedge \wedge}\end{array}$ & $\begin{array}{l}1 \\
1 \\
2 \\
24 \\
1 \\
12 \\
9 \\
1\end{array}$ & $\begin{array}{l}0 \\
0 \\
0 \\
2 \\
0 \\
1 \\
0 \\
0\end{array}$ & $\begin{array}{l}0 \\
0 \\
0 \\
0 \\
0 \\
0 \\
1 \\
0\end{array}$ & $\begin{array}{l}0 \\
0 \\
0 \\
0 \\
0 \\
0 \\
0 \\
0\end{array}$ & $\begin{array}{l}1 \\
1 \\
1 \\
18 \\
1 \\
8 \\
7 \\
0\end{array}$ & $\begin{array}{l}0 \\
0 \\
0 \\
0 \\
0 \\
0 \\
0 \\
0\end{array}$ & $\begin{array}{l}0 \\
0 \\
0 \\
0 \\
0 \\
0 \\
0 \\
0\end{array}$ & $\begin{array}{l}0 \\
0 \\
0 \\
0 \\
0 \\
0 \\
0 \\
0\end{array}$ & $\begin{array}{l}0 \\
0 \\
1 \\
0 \\
0 \\
0 \\
0 \\
0\end{array}$ & $\begin{array}{l}1 \\
1 \\
2 \\
20 \\
1 \\
9 \\
9 \\
0\end{array}$ \\
\hline $\begin{array}{l}\text { XLIV. Family Pholcidae C. L. Koch, } 1851 \\
\text { 279. Artema Walckenaer, } 1837 \\
\text { 280. Belisana Thorell, } 1898 \\
\text { 281. Crossopriza Simon, } 1893 \\
\text { 282. Holocneminus Berland, } 1942 \\
\text { 283. Pholciella Roewer, } 1960 \# \$ \\
\text { 284. Pholcoides Roewer, } 1960 \# \$ \\
\text { 285. Pholcus Walckenaer, } 1805 \\
\text { 286. Smeringopus Simon, } 1890 \\
\text { 287. Wanniyala Huber \& Benjamin, 2005@ }\end{array}$ & $\begin{array}{l}2 \\
5 \\
1 \\
1 \\
1 \\
1 \\
6 \\
1 \\
2\end{array}$ & $\begin{array}{l}1 \\
0 \\
0 \\
0 \\
1 \\
1 \\
0 \\
0 \\
0\end{array}$ & $\begin{array}{l}0 \\
0 \\
0 \\
0 \\
0 \\
0 \\
0 \\
0 \\
0\end{array}$ & $\begin{array}{l}0 \\
0 \\
0 \\
0 \\
0 \\
0 \\
0 \\
0 \\
0\end{array}$ & $\begin{array}{l}0 \\
2 \\
0 \\
0 \\
0 \\
0 \\
1 \\
0 \\
0\end{array}$ & $\begin{array}{l}0 \\
0 \\
0 \\
0 \\
0 \\
0 \\
0 \\
0 \\
0\end{array}$ & $\begin{array}{l}0 \\
0 \\
0 \\
0 \\
0 \\
0 \\
0 \\
0 \\
0\end{array}$ & $\begin{array}{l}0 \\
0 \\
0 \\
0 \\
0 \\
0 \\
0 \\
0 \\
0\end{array}$ & $\begin{array}{l}0 \\
3 \\
0 \\
0 \\
0 \\
0 \\
2 \\
0 \\
2\end{array}$ & $\begin{array}{l}1 \\
5 \\
0 \\
0 \\
1 \\
1 \\
3 \\
0 \\
2\end{array}$ \\
\hline $\begin{array}{l}\text { XLV. Family Pimoidae Wunderlich, } 1986 \\
\text { 288. Pimoa Chamberlin \& Ivie, } 1943\end{array}$ & 5 & 0 & 0 & 0 & 3 & 0 & 2 & 0 & 0 & 5 \\
\hline $\begin{array}{l}\text { XLVI. Family Pisauridae Simon, } 1890 \\
\text { 289. Dendrolycosa Doleschall, } 1859 \\
\text { 290. Dolomedes Latreille, } 1804 \\
\text { 291. Eucamptopus Pocock, } 1900{ }^{* \star} \\
\text { 292. Euprosthenops Pocock, } 1897\end{array}$ & $\begin{array}{l}2 \\
2 \\
1 \\
1\end{array}$ & $\begin{array}{l}0 \\
0 \\
0 \\
0\end{array}$ & $\begin{array}{l}0 \\
0 \\
0 \\
0\end{array}$ & $\begin{array}{l}0 \\
0 \\
0 \\
0\end{array}$ & $\begin{array}{l}2 \\
0 \\
1 \\
1\end{array}$ & $\begin{array}{l}0 \\
0 \\
0 \\
0\end{array}$ & $\begin{array}{l}0 \\
0 \\
0 \\
0\end{array}$ & $\begin{array}{l}0 \\
0 \\
0 \\
0\end{array}$ & $\begin{array}{l}0 \\
1 \\
0 \\
0\end{array}$ & $\begin{array}{l}2 \\
1 \\
1 \\
1\end{array}$ \\
\hline
\end{tabular}




\begin{tabular}{|c|c|c|c|c|c|c|c|c|c|c|}
\hline \multirow[t]{2}{*}{ Genus } & \multirow{2}{*}{$\begin{array}{l}\text { Total } \\
\text { spp. }\end{array}$} & \multirow[b]{2}{*}{ AF } & \multirow[b]{2}{*}{ BA } & \multirow[b]{2}{*}{$\mathrm{BH}$} & \multicolumn{3}{|c|}{ No. of Endemic Species } & & & \\
\hline & & & & & IN & MA & NE & PA & SL & SA \\
\hline 293. Hygropoda Thorell, 1894 & 1 & 0 & 0 & 0 & 0 & 0 & 0 & 0 & 0 & 0 \\
\hline 294. Nilus O.P.-Cambridge, 1876 & 2 & 0 & 0 & 0 & 2 & 0 & 0 & 0 & 0 & 2 \\
\hline 295. Perenethis L. Koch, 1878 & 2 & 0 & 0 & 0 & 0 & 0 & 0 & 0 & 0 & 1 \\
\hline 296. Pisaura Simon, 1885 & 6 & 0 & 0 & 0 & 5 & 0 & 0 & 0 & 0 & 5 \\
\hline 297. Thalassius Simon, 1885 & 2 & 0 & 0 & 0 & 0 & 0 & 0 & 0 & 0 & 0 \\
\hline 298. Tinus F. O. P.-Cambridge, 1901 & 2 & 0 & 0 & 0 & 2 & 0 & 0 & 0 & 0 & 2 \\
\hline XLVII. Family Prodidomidae Simon, 1884 & & & & & & & & & & \\
\hline 299. Prodidomus Hentz, 1847 & 8 & 0 & 0 & 0 & 7 & 0 & 0 & 1 & 0 & 8 \\
\hline 300. Zimiris Simon, 1882 & 2 & 0 & 0 & 0 & 0 & 0 & 0 & 0 & 0 & 0 \\
\hline XLVIII. Family Psechridae Simon, 1890 & & & & & & & & & & \\
\hline 301. Fecenia Simon, 1887 & 2 & 0 & 0 & 0 & 1 & 0 & 0 & 0 & 0 & 1 \\
\hline 302. Psechrus Thorell, 1878 & 4 & 0 & 0 & 0 & 0 & 0 & 1 & 0 & 0 & 2 \\
\hline XLIX. Family Salticidae Blackwall, 1841 & & & & & & & & & & \\
\hline 303. Aelurillus Simon, 1884 & 5 & 0 & 0 & 0 & 2 & 0 & 0 & 0 & 1 & 5 \\
\hline 304. Akela Peckham \& Peckham, 1896 & 1 & 0 & 0 & 0 & 0 & 0 & 0 & 1 & 0 & 1 \\
\hline 305. Asemonea O.P.-Cambridge, 1869 & 2 & 0 & 0 & 0 & 1 & 0 & 0 & 0 & 0 & 1 \\
\hline 306. Ballognatha Caporiacco, $1935^{\# \wedge \wedge}$ & 1 & 0 & 0 & 0 & 0 & 0 & 0 & 0 & 0 & 0 \\
\hline 307. Ballus C. L. Koch, 1850 & 2 & 0 & 0 & 0 & 0 & 0 & 0 & 0 & 2 & 2 \\
\hline 308. Bellota Peckham \& Peckham, 1892 & 2 & 0 & 0 & 0 & 0 & 0 & 0 & 2 & 0 & 2 \\
\hline 309. Bianor Peckham \& Peckham, 1886 & 5 & 0 & 0 & 0 & 0 & 0 & 0 & 0 & 0 & 1 \\
\hline 310. Brettus Thorell, 1895 & 3 & 0 & 0 & 0 & 0 & 0 & 0 & 0 & 1 & 2 \\
\hline 311. Bristowia Reimoser, 1934 & 1 & 0 & 0 & 0 & 0 & 0 & 0 & 0 & 0 & 0 \\
\hline 312. Carrhotus Thorell, 1891 & 9 & 0 & 0 & 2 & 0 & 0 & 3 & 0 & 1 & 6 \\
\hline 313. Chalcoscirtus Bertkau, 1880 & 3 & 0 & 0 & 0 & 0 & 0 & 0 & 0 & 0 & 0 \\
\hline 314. Chalcotropis Simon, 1902 & 1 & 0 & 0 & 0 & 1 & 0 & 0 & 0 & 0 & 1 \\
\hline 315. Chinattus Logunov, 1999 & 1 & 0 & 0 & 0 & 0 & 0 & 1 & 0 & 0 & 1 \\
\hline 316. Chrysilla Thorell, 1887 & 1 & 0 & 0 & 0 & 0 & 0 & 0 & 1 & 0 & 1 \\
\hline 317. Colaxes Simon, $1900 * *$ & 3 & 0 & 0 & 0 & 1 & 0 & 0 & 0 & 2 & 3 \\
\hline 318. Cosmophasis Simon, 1901 & 3 & 0 & 0 & 0 & 1 & 0 & 0 & 1 & 1 & 3 \\
\hline 319. Cotinusa Simon, 1900 & 1 & 0 & 0 & 0 & 0 & 0 & 0 & 1 & 0 & 1 \\
\hline 320. Curubis Simon, 1902 ** & 4 & 0 & 0 & 0 & 1 & 0 & 0 & 0 & 3 & 4 \\
\hline 321. Cyrba Simon, 1876 & 1 & 0 & 0 & 0 & 0 & 0 & 0 & 0 & 0 & 0 \\
\hline 322. Cytaea Keyserling, 1882 & 1 & 0 & 0 & 0 & 1 & 0 & 0 & 0 & 0 & 1 \\
\hline 323. Dendryphantes C. L. Koch, 1837 & 2 & 1 & 0 & 0 & 0 & 0 & 0 & 0 & 0 & 1 \\
\hline 324. Dexippus Thorell, 1891 & 1 & 0 & 0 & 0 & 1 & 0 & 0 & 0 & 0 & 1 \\
\hline 325. Dolichoneon Caporiacco, $1935^{\# \wedge \wedge}$ & 1 & 0 & 0 & 0 & 0 & 0 & 0 & 0 & 0 & 0 \\
\hline 326. Epeus Peckham \& Peckham, 1886 & 3 & 0 & 0 & 0 & 3 & 0 & 0 & 0 & 0 & 3 \\
\hline 327. Epidelaxia Simon, $1902 @$ & 3 & 0 & 0 & 0 & 0 & 0 & 0 & 0 & 3 & 3 \\
\hline 328. Epocilla Thorell, 1887 & 2 & 0 & 0 & 0 & 1 & 0 & 0 & 0 & 0 & 1 \\
\hline 329. Euophrys C.L. Koch, 1834 & 11 & 0 & 0 & 0 & 2 & 0 & 3 & 3 & 0 & 8 \\
\hline 330. Euryattus Thorell, 1881 & 2 & 0 & 0 & 0 & 0 & 0 & 0 & 0 & 1 & 1 \\
\hline 331. Evarcha Simon, 1902 & 4 & 2 & 0 & 0 & 0 & 0 & 0 & 0 & 0 & 2 \\
\hline 332. Flacillula Strand, 1932 & 2 & 0 & 0 & 0 & 0 & 0 & 0 & 1 & 1 & 2 \\
\hline 333. Freya C. L. Koch, 1850 & 1 & 0 & 0 & 0 & 0 & 0 & 0 & 1 & 0 & 1 \\
\hline 334. Gelotia Thorell, 1890 & 1 & 0 & 0 & 0 & 0 & 0 & 0 & 0 & 1 & 1 \\
\hline 335. Ghumattus Prószyn'ski, 1992 \#* & 1 & 0 & 0 & 0 & 1 & 0 & 0 & 0 & 0 & 1 \\
\hline 336. Habrocestoides Prószyn'ski, 1992 ** & 6 & 0 & 0 & 0 & 5 & 0 & 1 & 0 & 0 & 6 \\
\hline 337. Habrocestum Simon, 1876 & 2 & 0 & 0 & 0 & 0 & 0 & 0 & 2 & 0 & 2 \\
\hline 338. Harmochirus Simon, 1885 & 3 & 0 & 0 & 0 & 1 & 0 & 0 & 0 & 0 & 1 \\
\hline 339. Hasarius Simon, 1871 & 1 & 0 & 0 & 0 & 0 & 0 & 0 & 0 & 0 & 0 \\
\hline 340. Helicius Zabka, 1981 & 1 & 0 & 0 & 1 & 0 & 0 & 0 & 0 & 0 & 1 \\
\hline 341. Heliophanoides Prószyn'ski, 1992 ** & 3 & 0 & 0 & 1 & 2 & 0 & 0 & 0 & 0 & 3 \\
\hline 342. Heliophanus C. L. Koch, 1833 & 1 & 0 & 0 & 0 & 0 & 0 & 0 & 0 & 0 & 0 \\
\hline 343. Hindumanes Logunov, $2004^{\# *}$ & 1 & 0 & 0 & 0 & 1 & 0 & 0 & 0 & 0 & 1 \\
\hline 344. Hispo Simon, 1885 & 1 & 0 & 0 & 0 & 0 & 0 & 0 & 0 & 0 & 1 \\
\hline 345. Holcolaetis Simon, 1885 & 1 & 0 & 0 & 0 & 0 & 0 & 0 & 1 & 0 & 1 \\
\hline 346. Hyllus C. L. Koch, 1846 & 3 & 0 & 0 & 0 & 1 & 0 & 0 & 0 & 0 & 2 \\
\hline 347. Icius Simon, 1876 & 1 & 1 & 0 & 0 & 0 & 0 & 0 & 0 & 0 & 1 \\
\hline 348. Imperceptus Prószyn'ski, 1992 \#* & 1 & 0 & 0 & 0 & 1 & 0 & 0 & 0 & 0 & 1 \\
\hline 349. Irura Peckham \& Peckham, 1901 & 1 & 0 & 0 & 0 & 0 & 0 & 0 & 0 & 1 & 1 \\
\hline 350. Jajpurattus Prószyn'ski, 1992 \#* & 1 & 0 & 0 & 0 & 1 & 0 & 0 & 0 & 0 & 1 \\
\hline 351. Jollas Simon, 1901 & 1 & 0 & 0 & 0 & 0 & 0 & 0 & 1 & 0 & 1 \\
\hline 352. Langona Simon, 1901 & 7 & 1 & 0 & 0 & 4 & 0 & 0 & 0 & 0 & 5 \\
\hline 353. Madhyattus Prószyn'ski, $1992^{\# \star}$ & 1 & 0 & 0 & 0 & 1 & 0 & 0 & 0 & 0 & 1 \\
\hline 354. Marengo Peckham \& Peckham, 1892 & 5 & 0 & 0 & 0 & 0 & 0 & 0 & 0 & 5 & 5 \\
\hline 355. Marpissa C. L. Koch, 1846 & 20 & 0 & 1 & 0 & 14 & 0 & 0 & 5 & 0 & 20 \\
\hline 356. Menemerus Simon, 1868 & 6 & 0 & 0 & 0 & 1 & 0 & 0 & 1 & 0 & 2 \\
\hline 357. Modunda Simon, 1901 & 2 & 0 & 0 & 0 & 0 & 0 & 0 & 0 & 0 & 0 \\
\hline 358. Mogrus Simon, 1882 & 1 & 1 & 0 & 0 & 0 & 0 & 0 & 0 & 0 & 1 \\
\hline 359. Myrmarachne MacLeay, 1839 & 29 & 1 & 0 & 0 & 18 & 0 & 0 & 0 & 3 & 25 \\
\hline 360. Neobrettus Wanless, 1984 & 1 & 0 & 0 & 0 & 0 & 0 & 0 & 0 & 0 & 0 \\
\hline 361. Onomastus Simon, 1900 & 3 & 0 & 0 & 0 & 1 & 0 & 0 & 0 & 2 & 3 \\
\hline 362. Orissania Prószyn’ski, 1992 \#* & 1 & 0 & 0 & 0 & 1 & 0 & 0 & 0 & 0 & 1 \\
\hline
\end{tabular}




\begin{tabular}{|c|c|c|c|c|c|c|c|c|c|c|}
\hline \multirow[t]{2}{*}{ Genus } & \multirow{2}{*}{$\begin{array}{l}\text { Total } \\
\text { spp. }\end{array}$} & \multirow[b]{2}{*}{ AF } & \multirow[b]{2}{*}{ BA } & \multirow[b]{2}{*}{$\mathrm{BH}$} & \multicolumn{3}{|c|}{ No. of Endemic Species } & \multirow[b]{2}{*}{ PA } & \multirow[b]{2}{*}{ SL } & \\
\hline & & & & & IN & MA & NE & & & SA \\
\hline 363. Panachraesta Simon, 1900 \#@ & 1 & 0 & 0 & 0 & 0 & 0 & 0 & 0 & 1 & 1 \\
\hline 364. Pancorius Simon, 1902 & 10 & 0 & 0 & 3 & 4 & 0 & 1 & 0 & 0 & 8 \\
\hline 365. Pandisus Simon, 1900 & 1 & 0 & 0 & 0 & 1 & 0 & 0 & 0 & 0 & 1 \\
\hline 366. Panysinus Simon, 1901 & 2 & 0 & 0 & 0 & 1 & 0 & 0 & 0 & 1 & 2 \\
\hline 367. Pellenes Simon, 1876 & 3 & 1 & 0 & 0 & 0 & 0 & 0 & 1 & 0 & 2 \\
\hline 368. Penionomus Simon, 1903 & 1 & 0 & 0 & 0 & 0 & 0 & 0 & 1 & 0 & 1 \\
\hline 369. Phaeacius Simon, 1900 & 4 & 0 & 0 & 0 & 0 & 0 & 1 & 0 & 0 & 2 \\
\hline 370. Phausina Simon, 1902 & 3 & 0 & 0 & 0 & 0 & 0 & 0 & 0 & 3 & 3 \\
\hline 371. Phidippus C.L. Koch, 1846 & 7 & 0 & 1 & 0 & 6 & 0 & 0 & 0 & 0 & 7 \\
\hline 372. Phintella Strand, 1906 & 15 & 0 & 0 & 0 & 9 & 0 & 0 & 0 & 1 & 11 \\
\hline 373. Phlegra Simon, 1876 & 5 & 0 & 0 & 1 & 0 & 0 & 0 & 1 & 0 & 3 \\
\hline 374. Phyaces Simon, 1902 \#@ & 1 & 0 & 0 & 0 & 0 & 0 & 0 & 0 & 1 & 1 \\
\hline 375. Pilia Simon, 1902 & 2 & 0 & 0 & 0 & 1 & 0 & 0 & 1 & 0 & 2 \\
\hline 376. Piranthus Thorell, 1895 & 1 & 0 & 0 & 0 & 1 & 0 & 0 & 0 & 0 & 1 \\
\hline 377. Plexippoides Prószyn'ski, 1984 & 3 & 0 & 0 & 1 & 0 & 0 & 1 & 0 & 0 & 2 \\
\hline 378. Plexippus C.L. Koch, 1846 & 9 & 0 & 2 & 0 & 1 & 0 & 1 & 0 & 0 & 5 \\
\hline 379. Portia Karsch, 1878 & 4 & 0 & 0 & 0 & 0 & 0 & 0 & 0 & 0 & 0 \\
\hline 380. Pseudamycus Simon, 1885 & 2 & 0 & 0 & 1 & 1 & 0 & 0 & 0 & 0 & 2 \\
\hline 381. Pseudicius Simon, 1885 & 11 & 2 & 0 & 0 & 4 & 0 & 0 & 0 & 0 & 7 \\
\hline 382. Ptocasius Simon, 1885 & 1 & 0 & 0 & 0 & 0 & 0 & 0 & 0 & 1 & 1 \\
\hline 383. Pystira Simon, 1901 & 1 & 0 & 0 & 0 & 0 & 0 & 0 & 1 & 0 & 1 \\
\hline 384. Rafalus Prószyn'ski, 1999 & 1 & 0 & 0 & 1 & 0 & 0 & 0 & 0 & 0 & 1 \\
\hline 385. Rhene Thorell, 1869 & 16 & 0 & 0 & 0 & 11 & 0 & 0 & 0 & 0 & 13 \\
\hline 386. Saitis Simon, 1876 & 1 & 0 & 0 & 0 & 0 & 0 & 0 & 0 & 0 & 1 \\
\hline 387. Salticus Latreille, 1804 & 1 & 1 & 0 & 0 & 0 & 0 & 0 & 0 & 0 & 1 \\
\hline 388. Sigytes Simon, 1902 & 1 & 0 & 0 & 0 & 0 & 0 & 0 & 0 & 1 & 1 \\
\hline 389. Siler Simon, 1889 & 1 & 0 & 0 & 0 & 0 & 0 & 0 & 0 & 0 & 0 \\
\hline 390. Simaetha Thorell, 1881 & 3 & 0 & 0 & 0 & 0 & 0 & 0 & 0 & 3 & 3 \\
\hline 391. Similaria Prószyn’ski, $19922^{\# *}$ & 1 & 0 & 0 & 0 & 1 & 0 & 0 & 0 & 0 & 1 \\
\hline 392. Sitticus Simon, 1901 & 3 & 0 & 0 & 0 & 0 & 0 & 0 & 1 & 0 & 1 \\
\hline 393. Spartaeus Thorell, 1891 & 1 & 0 & 0 & 0 & 0 & 0 & 0 & 0 & 0 & 0 \\
\hline 394. Stagetillus Simon, 1885 & 1 & 0 & 0 & 0 & 0 & 0 & 0 & 0 & 1 & 1 \\
\hline 395. Stenaelurillus Simon, 1885 & 1 & 0 & 0 & 0 & 1 & 0 & 0 & 0 & 0 & 1 \\
\hline 396. Stergusa Simon, 1889 & 3 & 0 & 0 & 0 & 0 & 0 & 0 & 0 & 3 & 3 \\
\hline 397. Synagelides Strand, 1906 & 16 & 0 & 0 & 2 & 0 & 0 & 14 & 0 & 0 & 16 \\
\hline 398. Tamigalesus Zabka, 1988 \#@ & 1 & 0 & 0 & 0 & 0 & 0 & 0 & 0 & 1 & 1 \\
\hline 399. Telamonia Thorell, 1887 & 7 & 0 & 0 & 3 & 2 & 0 & 0 & 0 & 1 & 6 \\
\hline 400. Thiania C.L. Koch, 1846 & 3 & 0 & 0 & 0 & 0 & 0 & 0 & 1 & 0 & 1 \\
\hline 401. Thyene Simon, 1885 & 1 & 0 & 0 & 0 & 0 & 0 & 0 & 0 & 0 & 0 \\
\hline 402. Uroballus Simon, 1902 & 2 & 0 & 0 & 0 & 0 & 0 & 0 & 0 & 2 & 2 \\
\hline 403. Viciria Thorell, 1877 & 4 & 0 & 0 & 0 & 3 & 0 & 0 & 0 & 1 & 4 \\
\hline 404. Yaginumaella Prószyn'ski, 1979 & 27 & 0 & 0 & 21 & 1 & 0 & 1 & 0 & 0 & 23 \\
\hline 405. Yllenus Simon, 1868 & 2 & 0 & 0 & 0 & 2 & 0 & 0 & 0 & 0 & 2 \\
\hline 406. Zeuxippus Thorell, 1891 & 2 & 0 & 0 & 0 & 1 & 0 & 0 & 0 & 0 & 1 \\
\hline 407. Zygoballus Peckham \& Peckham, 1885 & & 0 & 0 & 0 & 3 & 0 & 0 & 0 & 0 & 3 \\
\hline $\begin{array}{l}\text { L. Family Scytodidae Blackwall, } 1864 \\
\text { 408. Scytodes Latreille, } 1804\end{array}$ & 12 & 1 & 0 & 0 & 3 & 0 & 0 & 1 & 0 & 7 \\
\hline LI. Family Segestriidae Simon, 1893 & & & & & & & & & & \\
\hline 409. Ariadna Audouin, 1826 & 3 & 0 & 0 & 0 & 1 & 0 & 0 & 0 & 2 & 3 \\
\hline 410. Segestria Latreille, 1804 & 1 & 0 & 0 & 0 & 1 & 0 & 0 & 0 & 0 & 1 \\
\hline LII. Family Selenopidae Simon, 1897 & & & & & & & & & & \\
\hline 411. Selenops Latreille, 1819 & 6 & 0 & 0 & 0 & 5 & 0 & 0 & 0 & 0 & 5 \\
\hline LIII. Family Sicariidae Keyserling, 1880 & & & & & & & & & & \\
\hline 412. Loxosceles Heineken \& Lowe, 1832 & 1 & 0 & 0 & 0 & 0 & 0 & 0 & 0 & 0 & 0 \\
\hline LIV. Family Sparassidae Bertkau, 1872 & & & & & & & & & & \\
\hline 413. Bhutaniella Jäger, 2000 & 6 & 0 & 0 & 3 & 1 & 0 & 2 & 0 & 0 & 6 \\
\hline 414. Eusparassus Simon, 1903 & 4 & 1 & 0 & 0 & 0 & 0 & 0 & 0 & 0 & 1 \\
\hline 415. Gnathopalystes Rainbow, 1899 & 1 & 0 & 0 & 0 & 0 & 0 & 0 & 0 & 0 & 0 \\
\hline 416. Heteropoda Latreille, 1804 & 36 & 1 & 0 & 0 & 25 & 0 & 0 & 0 & 3 & 31 \\
\hline 417. Martensopoda Jäger, 2006 & 2 & 0 & 0 & 0 & 2 & 0 & 0 & 0 & 0 & 2 \\
\hline 418. Olios Walckenaer, 1837 & 31 & 0 & 0 & 0 & 18 & 0 & 0 & 1 & 3 & 25 \\
\hline 419. Palystes L. Koch, 1875 & 1 & 0 & 0 & 0 & 1 & 0 & 0 & 0 & 0 & 1 \\
\hline 420. Pandercetes L. Koch, 1875 & 3 & 0 & 0 & 0 & 1 & 0 & 0 & 0 & 0 & 2 \\
\hline 421. Pseudopoda Jäger, 2000 & 39 & 0 & 0 & 2 & 5 & 0 & 30 & 0 & 0 & 38 \\
\hline 422. Rhitymna Simon, 1897 & 1 & 0 & 0 & 0 & 0 & 0 & 0 & 0 & 1 & 1 \\
\hline 423. Sivalicus Dyal, $1957 \# \$ \$$ & 1 & 0 & 0 & 0 & 0 & 0 & 0 & 1 & 0 & 1 \\
\hline 424. Spariolenus Simon, 1880 & 3 & 0 & 0 & 0 & 1 & 0 & 0 & 0 & 1 & 3 \\
\hline 425. Stasina Simon, 1877 & 2 & 0 & 0 & 0 & 0 & 0 & 0 & 0 & 2 & 2 \\
\hline 426. Thelcticopis Karsch, 1884 & 11 & 0 & 0 & 0 & 7 & 0 & 0 & 2 & 1 & 10 \\
\hline $\begin{array}{l}\text { LV. Family Stenochilidae Thorell, } 1873 \\
\text { 427. Stenochilus O.P.-Cambridge, } 1870\end{array}$ & 3 & 0 & 0 & 0 & 2 & 0 & 0 & 0 & 0 & 2 \\
\hline
\end{tabular}




\begin{tabular}{|c|c|c|c|c|c|c|c|c|c|c|}
\hline \multirow[t]{2}{*}{ Genus } & \multicolumn{4}{|l|}{ Total } & \multicolumn{6}{|c|}{ No. of Endemic Species } \\
\hline & spp. & AF & BA & BH & IN & MA & $\mathrm{NE}$ & PA & SL & SA \\
\hline \multicolumn{11}{|c|}{ LVI. Family Tetrablemmidae O. P.-Cambridge, 1873} \\
\hline 428. Brignoliella Shear, 1978 & 4 & 0 & 0 & 0 & 1 & 0 & 1 & 0 & 2 & 4 \\
\hline 429. Choiroblemma Bourne, $1980^{*}$ & 2 & 0 & 0 & 0 & 2 & 0 & 0 & 0 & 0 & 2 \\
\hline 430. Gunasekara Lehtinen, 1981\#@ & 1 & 0 & 0 & 0 & 0 & 0 & 0 & 0 & 1 & 1 \\
\hline 431. Indicoblemma Bourne, 1980 & 1 & 0 & 0 & 0 & 1 & 0 & 0 & 0 & 0 & 1 \\
\hline 432. Pahanga Shear, 1979 & 1 & 0 & 0 & 0 & 0 & 0 & 0 & 0 & 1 & 1 \\
\hline 433. Shearella Lehtinen, 1981 & 2 & 0 & 0 & 0 & 0 & 0 & 0 & 0 & 2 & 2 \\
\hline 434. Tetrablemma O.P.-Cambridge, 1873 & 5 & 0 & 0 & 0 & 3 & 0 & 1 & 0 & 0 & 5 \\
\hline \multicolumn{11}{|l|}{ LVII. Family Tetragnathidae Menge, 1866} \\
\hline 435. Atelidea Simon, 1895 & 1 & 0 & 0 & 0 & 0 & 0 & 0 & 0 & 1 & 1 \\
\hline 436. Atimiosa Simon, 1895 & 1 & 0 & 0 & 0 & 0 & 0 & 0 & 0 & 1 & 1 \\
\hline 437. Dolichognatha O. P.-Cambridge, 1869 & 1 & 0 & 0 & 0 & 0 & 0 & 0 & 0 & 1 & 1 \\
\hline 438. Dyschiriognatha Simon, 1893 & 1 & 0 & 0 & 0 & 0 & 0 & 0 & 0 & 0 & 0 \\
\hline 439. Guizygiella Zhu, Kim \& Song, 1997 & 1 & 0 & 0 & 0 & 0 & 0 & 0 & 0 & 0 & 0 \\
\hline 440. Leucauge White, 1841 & 14 & 0 & 0 & 0 & 7 & 0 & 0 & 0 & 1 & 8 \\
\hline 441. Meta C.L. Koch, 1836 & 2 & 0 & 0 & 0 & 2 & 0 & 0 & 0 & 0 & 2 \\
\hline 442. Opadometa Archer, 1951 & 1 & 0 & 0 & 0 & 0 & 0 & 0 & 0 & 0 & 0 \\
\hline 443. Orsinome Thorell, 1890 & 3 & 0 & 0 & 0 & 3 & 0 & 0 & 0 & 0 & 3 \\
\hline 444. Pachygnatha Sundevall, 1823 & 1 & 0 & 0 & 0 & 1 & 0 & 0 & 0 & 0 & 1 \\
\hline 445. Phonognatha Simon, 1894 & 1 & 0 & 0 & 0 & 1 & 0 & 0 & 0 & 0 & 1 \\
\hline 446. Schenkeliella Strand, 1934 \#@ & 1 & 0 & 0 & 0 & 0 & 0 & 0 & 0 & 1 & 1 \\
\hline 447. Tetragnatha Latreille, 1804 & 28 & 0 & 1 & 0 & 13 & 0 & 0 & 0 & 3 & 18 \\
\hline 448. Tylorida Simon, 1894 & 2 & 0 & 0 & 0 & 0 & 0 & 0 & 0 & 0 & 1 \\
\hline \multicolumn{11}{|l|}{ LVIII. Family Theraphosidae Thorell, 1870} \\
\hline 449. Annandaliella Hirst, $1909^{*}$ & 2 & 0 & 0 & 0 & 2 & 0 & 0 & 0 & 0 & 2 \\
\hline 450. Chilobrachys Karsch, 1891 & 10 & 0 & 0 & 0 & 6 & 0 & 0 & 0 & 1 & 8 \\
\hline 451. Haploclastus Simon, $1892^{*}$ & 8 & 0 & 0 & 0 & 8 & 0 & 0 & 0 & 0 & 8 \\
\hline 452. Haplocosmia Sch. \& v. Wir., $1996^{* *}$ & 2 & 0 & 0 & 0 & 1 & 0 & 1 & 0 & 0 & 2 \\
\hline 453. Ischnocolus Ausserer, 1871 & 2 & 0 & 0 & 0 & 2 & 0 & 0 & 0 & 0 & 2 \\
\hline 454. Lyrognathus Pocock, 1895 & 3 & 0 & 0 & 0 & 3 & 0 & 0 & 0 & 0 & 3 \\
\hline 455. Phlogiellus Pocock, 1897 & 1 & 0 & 0 & 0 & 1 & 0 & 0 & 0 & 0 & 1 \\
\hline 456. Plesiophrictus Pocock, 1899 & 14 & 0 & 0 & 0 & 13 & 0 & 0 & 0 & 1 & 14 \\
\hline 457. Poecilotheria Simon, 1885 ** & 15 & 0 & 0 & 0 & 8 & 0 & 0 & 0 & 7 & 15 \\
\hline 458. Selenocosmia Ausserer, 1871 & 4 & 0 & 0 & 0 & 2 & 0 & 0 & 1 & 0 & 3 \\
\hline 459. Thrigmopoeus Pocock, 1899 * & 2 & 0 & 0 & 0 & 2 & 0 & 0 & 0 & 0 & 2 \\
\hline \multicolumn{11}{|l|}{ LIX. Family Theridiidae Sundevall, 1833} \\
\hline 460. Achaearanea Strand, 1929 & 6 & 0 & 0 & 0 & 4 & 0 & 0 & 0 & 0 & 4 \\
\hline 461. Argyrodes Simon, 1864 & 15 & 0 & 0 & 0 & 10 & 0 & 0 & 0 & 1 & 12 \\
\hline 462. Ariamnes Thorell, 1869 & 2 & 0 & 0 & 0 & 1 & 0 & 0 & 0 & 0 & 2 \\
\hline 463. Cephalobares O. P.-Camb., 1870 \#@ & 1 & 0 & 0 & 0 & 0 & 0 & 0 & 0 & 1 & 1 \\
\hline 464. Chrysso O.P.-Cambridge, 1882 & 3 & 0 & 0 & 0 & 1 & 0 & 0 & 0 & 0 & 1 \\
\hline 465. Coscinida Simon, 1895 & 3 & 0 & 0 & 0 & 0 & 0 & 0 & 0 & 2 & 2 \\
\hline 466. Cyllognatha L. Koch, 1872 & 1 & 0 & 0 & 0 & 1 & 0 & 0 & 0 & 0 & 1 \\
\hline 467. Dipoena Thorell, 1869 & 3 & 0 & 0 & 0 & 0 & 0 & 0 & 2 & 1 & 3 \\
\hline 468. Emertonella Bryant, 1945 & 1 & 0 & 0 & 0 & 0 & 0 & 0 & 0 & 0 & 0 \\
\hline 469. Enoplognatha Pavesi, 1880 & 1 & 0 & 0 & 0 & 0 & 0 & 0 & 0 & 1 & 1 \\
\hline 470. Episinus Walckenaer, in Latreille, 1809 & 1 & 0 & 0 & 0 & 0 & 0 & 0 & 0 & 1 & 1 \\
\hline 471. Euryopis Menge, 1868 & 3 & 0 & 0 & 0 & 1 & 0 & 0 & 0 & 0 & 1 \\
\hline 472. Faiditus Keyserling, 1884 & 1 & 0 & 0 & 0 & 0 & 0 & 0 & 0 & 0 & 0 \\
\hline 473. Latrodectus Walckenaer, 1805 & 2 & 0 & 0 & 0 & 0 & 0 & 0 & 0 & 1 & 1 \\
\hline 474. Molione Thorell, 1892 & 1 & 0 & 0 & 0 & 0 & 0 & 0 & 0 & 1 & 1 \\
\hline 475. Moneta O.P.-Cambridge, 1870 & 1 & 0 & 0 & 0 & 1 & 0 & 0 & 0 & 0 & 1 \\
\hline 476. Phoroncidia Westwood, 1835 & 6 & 0 & 0 & 0 & 2 & 0 & 0 & 0 & 3 & 6 \\
\hline 477. Phycosoma O.P.-Cambridge, 1879 & 1 & 0 & 0 & 0 & 0 & 0 & 0 & 0 & 0 & 0 \\
\hline 478. Propostira Simon, $1894^{\star *}$ & 2 & 0 & 0 & 0 & 1 & 0 & 0 & 0 & 0 & 2 \\
\hline 479. Rhomphaea L. Koch, 1872 & 2 & 0 & 0 & 0 & 0 & 0 & 0 & 1 & 0 & 1 \\
\hline 480. Spintharus Hentz, 1850 & 1 & 0 & 0 & 0 & 0 & 0 & 0 & 1 & 0 & 1 \\
\hline 481. Steatoda Sundevall, 1833 & 3 & 0 & 0 & 0 & 1 & 0 & 0 & 0 & 0 & 1 \\
\hline 482. Theridion Walckenaer, 1805 & 23 & 0 & 0 & 0 & 12 & 0 & 0 & 0 & 6 & 18 \\
\hline 483. Theridula Emerton, 1882 & 2 & 0 & 0 & 0 & 2 & 0 & 0 & 0 & 0 & 2 \\
\hline 484. Thwaitesia O.P.-Cambridge, 1881 & 2 & 0 & 0 & 0 & 1 & 0 & 0 & 0 & 0 & 1 \\
\hline 485. Tomoxena Simon, 1895 & 1 & 0 & 0 & 0 & 1 & 0 & 0 & 0 & 0 & 1 \\
\hline LX. Family Theridiosomatidae Simon, 1881 & & & & & & & & & & \\
\hline 486. Andasta Simon, 1895 & 1 & 0 & 0 & 0 & 0 & 0 & 0 & 0 & 1 & 1 \\
\hline 487. Theridiosoma O. P.-Cambridge, 1879 & 1 & 0 & 0 & 0 & 0 & 0 & 0 & 0 & 1 & 1 \\
\hline 488. Wendilgarda Keyserling, 1886 & 1 & 0 & 0 & 0 & 0 & 0 & 0 & 0 & 0 & 0 \\
\hline LXI. Family Thomisidae Sundevall, 1833 & & & & & & & & & & \\
\hline 489. Amyciaea Simon, 1885 & 1 & 0 & 0 & 0 & 0 & 0 & 0 & 0 & 0 & 0 \\
\hline 490. Angaeus Thorell, 1881 & 1 & 0 & 0 & 0 & 1 & 0 & 0 & 0 & 0 & 1 \\
\hline 491. Ascurisoma Strand, $1928 \#$ & 1 & 0 & 0 & 0 & 0 & 0 & 0 & 0 & 0 & 0 \\
\hline 492. Boliscus Thorell, 1891 & 1 & 0 & 0 & 0 & 0 & 0 & 0 & 0 & 1 & 1 \\
\hline 493. Bomis L. Koch, 1874 & 3 & 0 & 0 & 0 & 3 & 0 & 0 & 0 & 0 & 3 \\
\hline
\end{tabular}




\begin{tabular}{|c|c|c|c|c|c|c|c|c|c|c|}
\hline \multirow[t]{2}{*}{ Genus } & \multicolumn{4}{|l|}{ Total } & \multicolumn{6}{|c|}{ No. of Endemic Species } \\
\hline & spp. & AF & BA & $\mathrm{BH}$ & IN & MA & NE & PA & SL & SA \\
\hline 494. Borboropactus Simon, 1884 & 2 & 0 & 0 & 0 & 1 & 0 & 0 & 0 & 1 & 2 \\
\hline 495. Camaricus Thorell, 1887 & 4 & 0 & 0 & 0 & 2 & 0 & 0 & 0 & 0 & 2 \\
\hline 496. Cymbacha L. Koch, 1874 & 1 & 0 & 0 & 0 & 0 & 0 & 0 & 0 & 1 & 1 \\
\hline 497. Demogenes Simon, 1895 & 1 & 0 & 0 & 0 & 1 & 0 & 0 & 0 & 0 & 1 \\
\hline 498. Diaea Thorell, 1869 & 6 & 0 & 0 & 0 & 2 & 0 & 0 & 2 & 1 & 5 \\
\hline 499. Dietopsa Strand, 1932* & 2 & 0 & 0 & 0 & 2 & 0 & 0 & 0 & 0 & 2 \\
\hline 500. Ebrechtella Dahl, 1907 & 4 & 0 & 0 & 0 & 0 & 0 & 0 & 1 & 0 & 1 \\
\hline 501. Epidius Thorell, 1877 & 2 & 0 & 0 & 0 & 0 & 0 & 0 & 0 & 1 & 1 \\
\hline 502. Henriksenia Lehtinen, 2005 & 1 & 0 & 0 & 0 & 0 & 0 & 0 & 0 & 0 & 0 \\
\hline 503. Heriaeus Simon, 1875 & 1 & 0 & 0 & 0 & 0 & 0 & 0 & 0 & 0 & 0 \\
\hline 504. Holopelus Simon, 1886 & 2 & 0 & 0 & 0 & 1 & 0 & 0 & 0 & 1 & 2 \\
\hline 505. Loxobates Thorell, 1877 & 3 & 0 & 0 & 1 & 2 & 0 & 0 & 0 & 0 & 3 \\
\hline 506. Lycopus Thorell, 1895 & 1 & 0 & 0 & 0 & 1 & 0 & 0 & 0 & 0 & 1 \\
\hline 507. Lysiteles Simon, 1895 & 19 & 0 & 0 & 4 & 2 & 0 & 4 & 0 & 0 & 13 \\
\hline 508. Massuria Thorell, 1887 & 2 & 0 & 0 & 0 & 2 & 0 & 0 & 0 & 0 & 2 \\
\hline 509. Mastira Thorell, 1891 & 2 & 0 & 0 & 0 & 2 & 0 & 0 & 0 & 0 & 2 \\
\hline 510. Misumena Latreille, 1804 & 5 & 0 & 0 & 0 & 5 & 0 & 0 & 0 & 0 & 5 \\
\hline 511. Misumenoides F.O.P.-Cambridge, 1900 & 1 & 0 & 0 & 0 & 1 & 0 & 0 & 0 & 0 & 1 \\
\hline 512. Misumenops F.O.P.-Cambridge, 1900 & 1 & 0 & 0 & 0 & 1 & 0 & 0 & 0 & 0 & 1 \\
\hline 513. Monaeses Thorell, 1869 & 8 & 0 & 0 & 0 & 4 & 0 & 0 & 0 & 2 & 6 \\
\hline 514. Oxytate L. Koch, 1878 & 7 & 0 & 0 & 1 & 3 & 0 & 0 & 0 & 2 & 6 \\
\hline 515. Ozyptila Simon, 1864 & 10 & 0 & 0 & 0 & 8 & 0 & 0 & 0 & 0 & 8 \\
\hline 516. Pagida Simon, 1895 & 1 & 0 & 0 & 0 & 0 & 0 & 0 & 0 & 1 & 1 \\
\hline 517. Parastrophius Simon, 1903 & 1 & 0 & 0 & 0 & 0 & 0 & 0 & 1 & 0 & 1 \\
\hline 518. Pasias Simon, 1895 & 2 & 0 & 0 & 0 & 2 & 0 & 0 & 0 & 0 & 2 \\
\hline 519. Peritraeus Simon, 1895\#@ & 1 & 0 & 0 & 0 & 0 & 0 & 0 & 0 & 1 & 1 \\
\hline 520. Philodamia Thorell, 1894 & 1 & 0 & 0 & 0 & 0 & 0 & 0 & 0 & 0 & 0 \\
\hline 521. Phrynarachne Thorell, 1869 & 5 & 0 & 0 & 0 & 2 & 0 & 0 & 0 & 2 & 4 \\
\hline 522. Pistius Simon, 1875 & 7 & 0 & 0 & 0 & 6 & 0 & 0 & 0 & 0 & 6 \\
\hline 523. Platythomisus Doleschall, 1859 & 2 & 0 & 0 & 0 & 2 & 0 & 0 & 0 & 0 & 2 \\
\hline 524. Runcinia Simon, 1875 & 11 & 0 & 0 & 0 & 6 & 0 & 0 & 0 & 0 & 7 \\
\hline 525. Stiphropus Gerstäcker, 1873 & 3 & 0 & 0 & 0 & 1 & 0 & 0 & 0 & 1 & 3 \\
\hline 526. Strigoplus Simon, 1885 & 4 & 0 & 0 & 0 & 3 & 0 & 0 & 0 & 0 & 3 \\
\hline 527. Synema Simon, 1864 & 3 & 0 & 0 & 1 & 1 & 0 & 0 & 0 & 0 & 2 \\
\hline 528. Tagulis Simon, 1895 & 1 & 0 & 0 & 0 & 0 & 0 & 0 & 0 & 1 & 1 \\
\hline 529. Talaus Simon, 1886 & 3 & 0 & 0 & 1 & 1 & 0 & 0 & 0 & 1 & 3 \\
\hline 530. Tarrocanus Simon, 1895 ** & 2 & 0 & 0 & 0 & 0 & 0 & 0 & 1 & 1 & 2 \\
\hline 531. Tharpyna L. Koch, 1874 & 2 & 0 & 0 & 0 & 2 & 0 & 0 & 0 & 0 & 2 \\
\hline 532. Thomisus Walckenaer, 1805 & 40 & 0 & 0 & 0 & 33 & 0 & 0 & 1 & 0 & 35 \\
\hline 533. Tmarus Simon, 1875 & 5 & 0 & 0 & 0 & 5 & 0 & 0 & 0 & 0 & 5 \\
\hline 534. Xysticus C.L. Koch, 1835 & 30 & 2 & 0 & 0 & 17 & 0 & 3 & 0 & 0 & 25 \\
\hline LXII. Family Titanoecidae Lehtinen, 1967 & & & & & & & & & & \\
\hline 535. Anuvinda Lehtinen, $1967^{\# \star}$ & 1 & 0 & 0 & 0 & 1 & 0 & 0 & 0 & 0 & 1 \\
\hline 536. Pandava Lehtinen, 1967 & 1 & 0 & 0 & 0 & 0 & 0 & 0 & 0 & 0 & 0 \\
\hline $\begin{array}{l}\text { LXIII. Family Trochanteriidae Karsch, } 1879 \\
\text { 537. Plator Simon, } 1880\end{array}$ & & & & & & & & & & \\
\hline 537. Plator Simon, 1880 & 5 & 0 & 0 & 0 & 4 & 0 & 0 & 0 & 0 & 4 \\
\hline $\begin{array}{l}\text { LXIV. Family Uloboridae Thorell, } 1869 \\
\text { 538. Hyptiotes Walckenaer, } 1837\end{array}$ & 3 & 0 & 0 & 0 & 2 & 0 & 0 & 0 & 1 & 3 \\
\hline 539. Miagrammopes O.P.-Cambridge, 1870 & 10 & 0 & 0 & 0 & 8 & 0 & 0 & 0 & 1 & 10 \\
\hline 540. Philoponella Mello-Leitão, 1917 & 1 & 0 & 0 & 0 & 1 & 0 & 0 & 0 & 0 & 1 \\
\hline 541. Uloborus Latreille, 1806 & 10 & 0 & 0 & 0 & 9 & 0 & 0 & 0 & 1 & 10 \\
\hline 542. Zosis Walckenaer, 1842 & 1 & 0 & 0 & 0 & 0 & 0 & 0 & 0 & 0 & 0 \\
\hline LXV. Family Zodariidae Thorell, 1881 & & & & & & & & & & \\
\hline 543. Asceua Thorell, 1887 & 1 & 0 & 0 & 0 & 1 & 0 & 0 & 0 & 0 & 1 \\
\hline 544. Capheris Simon, 1893 & 3 & 0 & 0 & 0 & 3 & 0 & 0 & 0 & 0 & 3 \\
\hline 545. Hermippus Simon, 1893 & 2 & 0 & 0 & 0 & 1 & 0 & 0 & 0 & 0 & 2 \\
\hline 546. Lutica Marx, 1891 & 4 & 0 & 0 & 0 & 4 & 0 & 0 & 0 & 0 & 4 \\
\hline 547. Storena Walckenaer, 1805 & 12 & 0 & 0 & 0 & 8 & 0 & 4 & 0 & 0 & 12 \\
\hline 548. Storenomorpha Simon, 1884 & 1 & 0 & 0 & 0 & 1 & 0 & 0 & 0 & 0 & 1 \\
\hline 549. Suffasia Jocqué, 1991** & 6 & 0 & 0 & 0 & 1 & 0 & 3 & 0 & 2 & 6 \\
\hline 550. Zodarion Walckenaer, 1826 & 1 & 1 & 0 & 0 & 0 & 0 & 0 & 0 & 0 & 1 \\
\hline $\begin{array}{l}\text { LXVI. Family Zorocratidae Dahl, } 1913 \\
\text { 551. CampostichommaKarsch, 1891"@ }\end{array}$ & 1 & 0 & 0 & 0 & 0 & 0 & 0 & 0 & 1 & 1 \\
\hline LXVII. Family Zoropsidae Bertkau, 1882 & & & & & & & & & & \\
\hline 552. Devendra Lehtinen, $1967 @$ & 3 & 0 & 0 & 0 & 0 & 0 & 0 & 0 & 3 & 3 \\
\hline Total & 2299 & 95 & 18 & 71 & 1053 & 0 & 176 & 72 & 246 & 1830 \\
\hline
\end{tabular}

AF: Afghanistan, BA: Bangladesh, BH: Bhutan; IN: India, MA: Maldives, NE: Nepal, PA: Pakistan, SL: Sri Lanka; SA: South Asia $\$$ - Genus endemic to Afghanistan, ${ }^{\wedge}$ - Genus endemic to Bhutan, ${ }^{*}$ - Genus endemic to India, \% - Genus endemic to Nepal, $\$ \$$ - Genus endemic to Pakistan, @ _ Genus endemic to Sri Lanka, $\wedge^{\wedge}$ - Genus known only from Karakorum, @@ - Genus known only from Himalaya, ${ }^{* *}$ - Genus endemic to South Asia, \# - Monotypic genus, \#\#- Monotypic family 


\section{Checklist 1. Spiders of Afghanistan}

\section{FAMILY CORINNIDAE KARSCH, 1880}

Comments: Considered valid by Wunderlich (1986) so genera listed by Brignoli under Castianeirinae and Corinninae (Clubionidae) are treated here and also genera listed by Roewer under the Corinninae have been apportioned to the Corinnidae (Platnick, 2006).

I.a. Genus Castianeira Keyserling, 1879

1. Castianeira rugosa Denis, 1958

Comments: Endemic to Afghanistan

I.b. Genus Corinnomma Karsch, 1880

1. Corinnomma afghanicum Roewer, 1962

Comments: Endemic to Afghanistan

II. FAMILY CYRTAUCHENIIDAE SIMON, 1892

II.a. Genus Anemesia Pocock, 1895

1. Anemesia tubifex (Pocock, 1889)

Synonym: Nemesia tubifex Pocock, 1889

Comments: Endemic to Afghanistan. Nemesia tubifex

was transferred to genus Anemesia by Pocock, (1895).

\section{FAMILY DYSDERIDAE C.L. KOCH, 1837}

III.a. Genus Dysdera Latreille, 1804

1. Dysdera afghana Denis, 1958

Comments: Endemic to Afghanistan. Deeleman-

Reinhold \& Deeleman (1988), removed Dysdera afghana from synonym of Dysdera zarudnyi.

2. Dysdera zarudnyi Charitonov, 1956

Distribution: Central Asia, Afghanistan

IV. FAMILY ERESIDAE C.L. KOCH, 1851

IV.a. Genus Eresus Walckenaer, 1805

1. Eresus walckenaeri Brullé, 1832

Synonym: Eresus walckenaer Brullé, 1832

Eresus audouin Brullé, 1832

Eresus theis Brullé, 1832

Eresus ctenizoides C. L. Koch, 1836

Eresus luridus C. L. Koch, 1836

Eresus puniceus C. L. Koch, 1837

Eresus pruinosus C. L. Koch, 1846

Eresus siculus Lucas, 1864

Erythrophora punicea Simon, 1864

Distribution: Mediterranean

1.a Eresus walckenaeri moerens C.L. Koch, 1846 Comments: Endemic to Afghanistan. Roewer (1962) removed from species and placed it as subspecies of Eresus walckenaeri.

\section{FAMILY FILISTATIDAE AUSSERER, 1867}

V.a. Genus Filistata Latreille, 1810

1. Filistata afghana Roewer, 1962

Comments: Endemic to Afghanistan

V.b. Genus Pritha Lehtinen, 1967

1. Pritha lindbergi (Roewer, 1962)

Synonym: Filistata lindbergi Roewer, 1962 Comments: Endemic to Afghanistan

\section{FAMILY GNAPHOSIDAE POCOCK, 1898}

VI.a. Genus Anagraphis Simon, 1893

Comments: This genus was transferred from family Prodidomidae to family Gnaphosidae by Platnick \& Baehr (2006)

1. Anagraphis maculosa Denis, 1958

Comments: Endemic to Afghanistan

VI.b. Genus Asiabadus Roewer, 1961 Comments: This is monotypic genus.
1. Asiabadus asiaticus (Charitonov, 1946) Synonym: Scotophaeus asiaticus Charitonov, 1946

2. Asiabadus hamiger Roewer, 1961

Distribution: Central Asia, Afghanistan

Comments: Scotophaeus asiaticus was transferred to genus Asiabadus by Ovtsharenko \& Fet (1980).

VI.c. Genus Berlandina Dalmas, 1922

1. Berlandina denisi Roewer, 1961

Comments: Endemic to Afghanistan

2. Berlandina propinqua Roewer, 1961

Comments: Endemic to Afghanistan

VI.d. Genus Drassodes Westring, 1851

1. Drassodes afghanus Roewer, 1961

Comments: Endemic to Afghanistan

2. Drassodes bendamiranus Roewer, 1961

Comments: Endemic to Afghanistan

3. Drassodes bicurvatus Roewer, 1961

Comments: Endemic to Afghanistan

4. Drassodes lindbergi Roewer, 1961

Comments: Endemic to Afghanistan

5. Drassodes lividus Denis, 1958

Comments: Endemic to Afghanistan

6. Drassodes robatus Roewer, 1961

Comments: Endemic to Afghanistan

7. Drassodes termezius Roewer, 1961

Comments: Endemic to Afghanistan

8. Drassodes tiritschensis Miller \& Buchar, 1972

Comments: Endemic to Afghanistan

VI.e. Genus Gnaphosa Latreille, 1804

1. Gnaphosa danieli Miller \& Buchar, 1972

Comments: Endemic to Afghanistan

2. Gnaphosa perplexa Denis, 1958

Comments: Endemic to Afghanistan

VI.f. Genus Herpyllus Hentz, 1832

1. Herpyllus lativulvus Denis, 1958

Comments: Endemic to Afghanistan

2. Herpyllus paropanisadensis Denis, 1958

Comments: Endemic to Afghanistan

3. Herpyllus proximus Denis, 1958

Distribution: Turkmenistan, Afghanistan

4. Herpyllus vicinus Denis, 1958

Comments: Endemic to Afghanistan

VI.g. Genus Kirmaka Roewer, 1961

Comment: This genus is monotypic and endemic to Afghanistan.

1. Kirmaka krausi Roewer, 1961

Comments: Endemic to Afghanistan

VI.h. Genus Micaria Westring, 1851

1. Micaria braendegaardi Denis, 1958

Comments: Endemic to Afghanistan

2. Micaria lindbergi Roewer, 1962

Comments: Endemic to Afghanistan. According to Wunderlich (1979), this species could be Micaria rossica.

3. Micaria pallens Denis, 1958

Comments: Endemic to Afghanistan. According to
Wunderlich (1979), this species could be Micaria rossica.

VI.i. Genus Minosiella Dalmas, 192 1. Minosiella intermedia Denis, 1958

Distribution: Central Asia, Afghanistan

VI.j. Genus Nomisia Dalmas, 1921

1. Nomisia kabuliana Roewer, 1961

Comments: Endemic to Afghanistan

VI.k. Genus Scotophaeus Simon, 1893

1. Scotophaeus afghanicus Roewer, 1961

Comments: Endemic to Afghanistan

2. Scotophaeus lindbergi Roewer, 1961

Comments: Endemic to Afghanistan

VI.I. Genus Siruasus Roewer, 1961

Comment: This genus is monotypic and endemic to Afghanistan.

1. Siruasus crassipalpus Roewer, 196

Comments: Endemic to Afghanistan

VI.m. Genus Zelotes Gistel, 1848

1. Zelotes anchoralis Denis, 1958

Comments: Endemic to Afghanistan

2. Zelotes bozbalus Roewer, 1961

Comments: Endemic to Afghanistan

3. Zelotes chotorus Roewer, 1961

Comments: Endemic to Afghanistan

4. Zelotes konarus Roewer, 196

Comments: Endemic to Afghanistan

5. Zelotes laghmanus Roewer, 1961

Comments: Endemic to Afghanistan

6. Zelotes planiger Roewer, 1961

Comments: Endemic to Afghanistan

7. Zelotes spinulosus Denis, 1958

Comments: Endemic to Afghanistan

VII. FAMILY HAHNIIDAE BERTKAU, 1878

VII.a. Genus Hahnia C.L. Koch, 1841

1. Hahnia spasskyi Denis, 1958

Synonym: Iberina spasskyi (Denis, 1958)

Comments: Endemic to Afghanistan. Original name reinstated by Brignoli (1983).

VIII. FAMILY HERSILIIDAE THORELL, 1870

VIII.a. Genus Hersiliola Thorell, 1870

1. Hersiliola afghanica Roewer, 1960

Distribution: Afghanistan, Turkmenistan

\section{FAMILY LINYPHIIDAE BLACKWALL, 1859}

IX.a. Genus Arachosinella Denis, 1958

1. Arachosinella strepens Denis, 1958

Distribution: Russia, Mongolia, Central Asia, Afghanistan

IX.b. Genus Lepthyphantes Menge, 1866

1. Lepthyphantes afghanus Denis, 1958

Comments: Endemic to Afghanistan

IX.c. Genus Mughiphantes Saaristo \& Tanasevitch, 1999

1. Mughiphantes hindukuschensis (Miller \& Buchar 1972)

Synonym: Lepthyphantes hindukuschensis Miller \& Buchar, 1972

Comments: Endemic to Afghanistan. Lepthyphantes hindukuschensis was transferred to genus Mughiphantes by Saaristo \& Tanasevitch (1999). 


\section{FAMILY LYCOSIDAE SUNDEVALL, 1833}

X.a. Genus Allocosa Banks, 1900

1. Allocosa manmaka Roewer, 1960

Comments: Endemic to Afghanistan

2. Allocosa pellita Roewer, 1960

Comments: Endemic to Afghanistan

3. Allocosa sangtoda Roewer, 1960

Comments: Endemic to Afghanistan

X.b. Genus Alopecosa Simon, 1885

1. Alopecosa lindbergi Roewer, 1960

Comments: Endemic to Afghanistan

2. Alopecosa nybelini Roewer, 1960 Comments: Endemic to Afghanistan

X.c. Genus Arctosa C.L. Koch, 1847 1. Arctosa bakva (Roewer, 1960)

Synonym: Arkalosula bakva Roewer, 1960

Comments: Endemic to Afghanistan. According to Platnick (2006), Arkalosula bakva is transferred to genus Arctosa.

2. Arctosa darountaha Roewer, 1960

Comments: Endemic to Afghanistan

3. Arctosa kadjahkaia Roewer, 1960

Synonym: Arctosa kadjakhaia Roewer, 1960

Comments: Endemic to Afghanistan

X.d. Genus Evippa Simon, 1882

Synonym: Evippella Strand, 1906

Comments: Evippella was synonymised with genus

Evippa by Alderweireldt (1991)

1. Evippa nigerrima (Miller \& Buchar, 1972)

Synonym: Evippella nigerrima Miller \& Buchar, 1972

Comments: Endemic to Afghanistan

X.e. Genus Geolycosa Montgomery, 1904

1. Geolycosa sexmaculata Roewer, 1960

Comments: Endemic to Afghanistan

X.f. Genus Hippasa Simon, 1885

1. Hippasa afghana Roewer, 1960

Comments: Endemic to Afghanistan

X.g. Genus Hogna Simon, 1885

Synonym: Genus Lycorma Simon, 1885

Comments: The genus Lycorma was synonymised with the genus Hogna by Wunderlich (1992).

1. Hogna bhougavia Roewer, 1960

Comments: Endemic to Afghanistan

X.h. Genus Lycosa Latreille, 1804

Synonym: Genus Allohogna Roewer, 1955

Comments: Allohogna was synonymised with genus

Lycosa by Fuhn \& Niculescu-Burlacu (1971).

1. Lycosa trichopus (Roewer, 1960)

Synonym: Allohogna trichopus Roewer, 1960

Comments: Endemic to Afghanistan

X.i. Genus Megarctosa Caporiacco, 1948

1. Megarctosa bamiana Roewer, 1960

Comments: Endemic to Afghanistan

X.j. Genus Pardosa C.L. Koch, 1847

1. Pardosa bendamira Roewer, 1960

Comments: Endemic to Afghanistan

2. Pardosa chahraka Roewer, 1960

Comments: Endemic to Afghanistan
3. Pardosa chindanda Roewer, 1960 Comments: Endemic to Afghanistan

4. Pardosa dalkhaba Roewer, 1960 Comments: Endemic to Afghanistan

5. Pardosa ghourbanda Roewer, 1960 Comments: Endemic to Afghanistan

6. Pardosa golbagha Roewer, 1960

Comments: Endemic to Afghanistan

7. Pardosa guerechka Roewer, 1960 Comments: Endemic to Afghanistan

8. Pardosa maimaneha Roewer, 1960 Comments: Endemic to Afghanistan

9. Pardosa pseudotorrentum Miller \& Buchar, 1972 Comments: Endemic to Afghanistan

10. Pardosa rhombisepta Roewer, 1960

Synonym: Pardosa rhombiseptum Roewer, 1960

Comments: Endemic to Afghanistan

X.k. Genus Schizocosa Chamberlin, 1904

Synonym: Genus Avicosa Chamberlin \& Ivie, 1942 Comments: The genus Avicosa was synonymised with the genus Schizocosa by Roewer (1955).

1. Schizocosa afghana (Roewer, 1960) Synonym: Avicosa afghana Roewer, 1960 Comments: Endemic to Afghanistan

2. Schizocosa salara (Roewer, 1960) Synonym: Avicosa salara Roewer, 1960 Comments: Endemic to Afghanistan

X.I. Genus Trochosula Roewer, 1960 1. Trochosula afghana Roewer, 1960 Comments: Endemic to Afghanistan

\section{FAMILY NESTICIDAE Simon, 1894}

XI.a. Genus Nesticus Thorell, 1869

1. Nesticus afghanus Roewer, 1962

Comments: Endemic to Afghanistan

2. Nesticus concolor Roewer, 1962

Comments: Endemic to Afghanistan

3. Nesticus lindbergi Roewer, 1962

Comments: Endemic to Afghanistan

XII. FAMILY OECOBIIDAE BLACKWALL, 1862

XII.a. Genus Uroctea Dufour, 1820

Comments: Tikader (1987) considered this genus in the family Urocteidae, which was a wrong placement.

Uroctea grossa Roewer, 1960

Comments: Endemic to Afghanistan

XIII. FAMILY OONOPIDAE SIMON, 1890

XIII.a. Genus Gamasomorpha Karsch, 1881

1. Gamasomorpha kabulensis Roewer, 1960

Comments: Endemic to Afghanistan

XIV. FAMILY PHILODROMIDAE THORELL, 1870

XIV.a. Genus Philodromus Walckenaer, 1826

Comments: Genus Philodromus was transferred from $\quad$ 2. Langona pallida Prószyn'ski, 1993

family Thomisidae to family Philodromidae by Homann (1975).

1. Philodromus populicola Denis, 1958

Comments: Endemic to Afghanistan

2. Philodromus punctatissimus Roewer, 1962

Comments: Endemic to Afghanistan

2. Evarcha darinurica Logunov, 2001
XIV.b. Genus Thanatus C.L. Koch, 1837

Comments: Removed from the Thomisidae and placed under Philodromidae by Homann (1975).

1. Thanatus denisi Brignoli, 1983

Synonym: Thanatus punctulatus Denis, 1958 Comments: Endemic to Afghanistan. Thanatus punctulatus was preoccupied by Taczanowski (1872) and thus, Brignoli (1983) provided replacement name.

XV. FAMILY PHOLCIDAE C.L. KOCH, 1851

XV.a. Genus Artema Walckenaer, 1837

1. Artema magna Roewer, 1960

Comments: Endemic to Afghanistan

XV.b. Genus Pholciella Roewer, 1960

Comments: It is a monotypic genus and endemic to Afghanistan.

1. Pholciella ziaretana Roewer, 1960

Comments: Endemic to Afghanistan

XV.c. Genus Pholcoides Roewer, 1960

Comments: It is a monotypic genus and endemic to Afghanistan.

1. Pholcoides afghana Roewer, 1960

Comments: Endemic to Afghanistan

XVI. FAMILY SALTICIDAE BLACKWALL, 1841

XVI.a. Genus Aelurillus Simon, 1884

1. Aelurillus logunovi Azarkina, 2004

Distribution: Afghanistan, Pakistan

Comments: Endemic to South Asia

XVI.b. Genus Bianor Peckham \& Peckham, 1886

1. Bianor punjabicus Logunov, 2001

Distribution: India, Afghanistan

Comments: Endemic to South Asia

XVI.c. Genus Dendryphantes C. L. Koch, 1837

1. Dendryphantes praeposterus Denis, 1958

Comments: Endemic to Afghanistan

XVI.d. Genus Evarcha Simon, 1902

1. Evarcha crinita Logunov \& Zamanpoore, 2005

Comments: Endemic to Afghanistan

Comments: Endemic to Afghanistan

XVI.e. Genus Heliophanus C. L. Koch, 1833

1. Heliophanus potanini Schenkel, 1963

Synonym: Menemerus fagei Schenkel, 1963

Heliophanus tribulosus Prószyn'ski, 1979

Distribution: Afghanistan, Central Asia, Mongolia, China

XVI.f. Genus Icius Simon, 1876

1. Icius abnormis Denis, 1958

Comments: Endemic to Afghanistan

XVI.g. Genus Langona Simon, 1901

1. Langona aperta (Denis, 1958)

Synonym: Aelurillus apertus Denis, 1958

Comments: Endemic to Afghanistan. Aelurillus apertus was transferred to genus Langona by Heciak \& Prószyn'ski (1983).

Distribution: Saudi Arabia, Afghanistan

XVI.h. Genus Mogrus Simon, 1882

1. Mogrus faizabadicus Andreeva, Kononenko \&

Prószyn'ski, 1981

Comments: Endemic to Afghanistan 
XVI.i. Genus Myrmarachne MacLeay, 1839 1. Myrmarachne palladia Denis, 1958 Comments: Endemic to Afghanistan

XVI.j. Genus Pellenes Simon, 1876

1. Pellenes lucidus Logunov \& Zamanpoore, 2005

Comments: Endemic to Afghanistan

XVI.k. Genus Plexippoides Prószyn'ski, 1984

1. Plexippoides flavescens (O.P.-Cambridge, 1872)

Synonym: Salticus flavescens O.P.-Cambridge, 1872

Menemerus flavescens (O.P.-Cambridge, 1872)

Yllenus starmühlneri Roewer, 1955

Evarcha afghana Roewer, 1962

Plexippoides starmuehlneri (Roewer, 1955)

Plexippoides arabicus Prószyn'ski, 1989

Menemerops flavescens (O. P.-Cambridge, 1872)

Menemerops afghanus (Roewer, 1962)

Menemerops sollistimus Wesolowska \& van Harten, 1994

Plexippoides afghanus (Roewer, 1962)

Distribution: Greece to Central Asia

Comments: Plexippoides afghanus was synonymised

with Plexippoides flavescens by Logunov \& Zamanpoore (2005).

XVI.I. Genus Pseudicius Simon, 1885

1. Pseudicius afghanicus (Andreeva, Heciak \&

Prószyn'ski, 1984)

Synonym: Icius afghanicus Andreeva, Heciak \& Prószyn'ski, 1984

Comments: Endemic to Afghanistan. Icius afghanicus was transferred to genus Pseudicius by Prószyn'ski (1990).

2. Pseudicius arabicus (Wesolowska \& van Harten, 1994) Synonym: Afraflacilla arabica Wesolowska \& van Harten, 1994

Pseudicius braunsi Wesolowska, 1996

Distribution: Yemen, Afghanistan

3. Pseudicius datuntatus Logunov \& Zamanpoore, 2005

Comments: Endemic to Afghanistan

4. Pseudicius frigidus (O.P.-Cambridge, 1885)

Synonyms: Menemerus frigidus O.P.-Cambridge, 1885

Phlegra icioides Simon, 1889

Icius icioides (Simon, 1889)

Icius frigidus (O.P.-Cambridge, 1885)

Distribution: Afghanistan, Pakistan, India, China

Comments: Icius icioides was synonymised with

Pseudicius frigidus by Andreeva et al. (1984).

XVI.m. Genus Salticus Latreille, 1804

1. Salticus afghanicus Logunov \& Zamanpoore, 2005

Comments: Endemic to Afghanistan

XVI.n. Genus Thyene Simon, 1885

1. Thyene imperialis (Rossi, 1846)

Synonym: Attus imperialis Rossi, 1846

Salticus moreletii Lucas, 1846

Attus regillus L. Koch, 1867

Attus argentoe-lineatus Simon, 1868

Attus moreletii (Lucas, 1846)

Marpessa imperialis (Rossi, 1846)

Thya imperialis (Rossi, 1846)

Thiene imperialis (Rossi, 1846)

Thyene moreleti (Lucas, 1846)

Thyene lindbergi Roewer, 1962

Thyene sinensis Schenkel, 1963

Distribution: Old World

Comments: Thyene lindbergi was synonymised with

Thyene imperialis by Logunov \& Zamanpoore (2005).

XVII. FAMILY SCYTODIDAE BLACKWALL, 1864

XVII.a. Genus Scytodes Latreille, 1804

1. Scytodes sexstriata Roewer, 1960
Comments: Endemic to Afghanistan

XVIII. FAMILY SPARASSIDAE BERTKAU, 1872 Comments: Considered senior synonym Heteropodidae by Jäger (1999).

XVIII.a. Genus Eusparassus Simon, 1903

1. Eusparassus fuscimanus Denis, 1958

Synonyms: Sparassus fuscimanus Levy, 1989

Comments: Endemic to Afghanistan

2. Eusparassus walckenaeri (Audouin, 1826)

Synonyms: Philodromus walckenaerii Audouin, 1826

Philodromus linnaei Audouin, 1826

Drassus civilis Wider, 1834

Ocypete tersa C. L. Koch, 1837

Sparassus doriae Simon, 1874

Sparassus cambridgii Simon, 1874

Sparassus validus Thorell, 1875

Sparassus cognatus O. P.-Cambridge, 1876

Sparassus extensipes Karsch, 1880

Sparassus walckenaerii (Audouin, 1826)

Sparassus tersus (C. L. Koch, 1837)

Sparassus fontanieri Simon, 1880

Sparassus linnaei (Audouin, 1826)

Eusparassus tersus Järvi, 1914

Heteropoda civilis (Wider, 1834)

Eusparassus kronebergi Denis, 1958

Distribution: Eastern Mediterranean to Afghanistan

XVIII.b. Genus Heteropoda Latreille, 1804

Synonyms: Genus Torania Simon, 1886

Genus Panaretus Simon, 1880

Comments: Genus Torania was synonymised with

genus Heteropoda by Jäger (2001) and genus

Panaretus was synonymised with genus Heteropoda

by Jäger (2002).

1. Heteropoda afghana Roewer, 1962

Distribution: Afghanistan, Pakistan, India

2. Heteropoda lindbergi Roewer, 1962

Comments: Endemic to Afghanistan

XIX. FAMILY THOMISIDAE SUNDEVALL, 1833

XIX.a. Genus Ozyptila Simon, 1864

Comments: According to Platnick (2006), genus name

Oxyptila used by Tikader and various other authors

is an unjustified emendation.

1. Ozyptila grisea Roewer, 1955

Distribution: Iran, Afghanistan

XIX.b. Genus Xysticus C.L. Koch, 1835

1. Xysticus lindbergi Roewer, 1962

Comments: Endemic to Afghanistan

2. Xysticus maculatipes Roewer, 1962

Comments: Endemic to Afghanistan

XX. FAMILY ZODARIIDAE THORELL, 1881

XX.a. Genus Zodarion Walckenaer, 1826

1. Zodarion lindbergi Roewer, 1960

Comments: Endemic to Afghanistan

\section{Afghanistan spider summary}

Number of families: 20

Number of Genera: 66

Number of Species: 113

Number of subspecies:

Number of Endemic Families: 0

Number of Endemic Genera: 4

Number of Endemic Species: 95

\section{Checklist 2. Spiders of Bangladesh}

I. FAMILY ARANEIDAE SIMON, 1895

of I.a. Genus Araneus Clerck, 1757

1. Araneus ellipticus (Tikader \& Bal, 1981)

Synonyms: Neoscona elliptica Tikader \& Bal, 1981

Neoscona griseomaculata Yin \& Wang, 1982

Distribution: India, Bangladesh, China

Comments: Grasshoff (1986) transferred Neoscona elliptica to genus Araneus.

I.b. Genus Argiope Audouin, 1826

1. Argiope minuta Karsch, 1879

Synonym: Argiope shillongensis Sinha, 1951

Distribution: India, Bangladesh, East Asia

Comments: Argiope shillongensis was synonymised with Argiope minuta by Levi (1983).

I.c. Genus Cyclosa Menge, 1866

1. Cyclosa bituberculata Biswas \& Raychaudhuri, 1998

Comments: Endemic to Bangladesh

2. Cyclosa confraga (Thorell, 1892)

Synonym: Epeira confraga Thorell, 1892

Distribution: India, Bangladesh to Malaysia

3. Cyclosa elongata Biswas \& Raychaudhuri, 1998 Comments: Endemic to Bangladesh

4. Cyclosa tropica Biswas \& Raychaudhuri, 1998

Comments: Endemic to Bangladesh

5. Cyclosa yaginumai Biswas \& Raychaudhuri, 1998 Comments: Endemic to Bangladesh

I.d. Genus Cyrtophora Simon, 1864

1. Cyrtophora lahirii Biswas \& Raychaudhuri, 2004

Comments: Endemic to Bangladesh

2. Cyrtophora nareshi Biswas \& Raychaudhuri, 2004 Comments: Endemic to Bangladesh

I.e. Genus Gea C.L. Koch, 1843

1. Gea subarmata Thorell, 1890

Synonyms: Gea catenulata Thorell, 1898

Gea brongersmai Chrysanthus, 1971

Gea corbetti Tikader, 1982

Distribution: India, Bangladesh to Philippines, New

Guinea

Comments: Gea corbetti was synonymised with Gea subarmata by Levi (1983).

I.f. Genus Neoscona Simon, 1864

1. Neoscona molemensis Tikader \& Bal, 1981

Distribution: Bangladesh, India to Philippines, Indonesia

\section{FAMILY CLUBIONIDAE WAGNER, 1887}

II.a. Genus Clubiona Latreille, 1804

1. Clubiona analis Thorell, 1895

Distribution: India, Bangladesh, Myanmar

2. Clubiona anwarae Biswas \& Raychaudhuri, 1996 Comments: Endemic to Bangladesh

3. Clubiona bagerhatensis Biswas \& Raychaudhuri, 1996

Comments: Endemic to Bangladesh

4. Clubiona drassodes O. P.-Cambridge, 1874

Synonym: Clubiona atwali Singh, 1970

Distribution: India, Bangladesh, China

Comments: Clubiona atwali was synonymised with

Clubiona drassodes by Barrion \& Litsinger (1995). 
5. Clubiona filicata O. P.-Cambridge, 1874

Synonym: Clubiona swatowensis Strand, 1907

Distribution: India, Bangladesh, Pakistan, China

Comments: According to Platnick (2006), this specie occurs in Pakistan.

6. Clubiona ludhianaensis Tikader, 1976

Distribution: India, Bangladesh

Comments: Endemic to South Asia

7. Clubiona mujibari Biswas \& Raychaudhuri, 1996 Comments: Endemic to Bangladesh

\section{FAMILY CORINNIDAE KARSCH, 1880}

Comments: Considered valid by Wunderlich (1986) so genera listed by Brignoli under Castianeirinae and Corinninae (Clubionidae) are treated here and also genera listed by Roewer under the Corinninae have been apportioned to the Corinnidae (Platnick, 2006).

III.a. Genus Castianeira Keyserling, 1879 1. Castianeira rugosa Denis, 1958

Comments: Endemic to Afghanistan

III.b. Genus Trachelas L. Koch, 1872

1. Trachelas devi Biswas \& Raychaudhuri, 2000

Comments: Endemic to Bangladesh

\section{FAMILY LINYPHIIDAE BLACKWALL, 1859}

IV.a. Genus Callitrichia Fage, 1936

1. Callitrichia formosana Oi, 1977

Synonym: Oedothorax formosanus Brignoli, 1983

Atypena formosana Tazoe, 1992

Distribution: Bangladesh to Japan

\section{FAMILY LIOCRANIDAE SIMON, 1897}

V.a. Genus Sphingius Thorell, 1890

Comments: This genus was originally described in the family Clubionidae. After a series of transfers, it was finally transferred to family Liocranidae by Deeleman-Reinhold (2001).

1. Sphingius barkudensis Gravely, 1931

Synonym: Sphingius barkudaensis Biswas \& Raychaudhuri, 2000

Distribution: Bangladesh, India

Comments: Endemic to South Asia

\section{FAMILY LYCOSIDAE SUNDEVALL, 1833}

VI.a. Genus Lycosa Latreille, 1804

Synonym: Genus Allohogna Roewer, 1955

Comments: Allohogna was synonymised with genus

Lycosa by Fuhn \& Niculescu-Burlacu (1971).

1. Lycosa mackenziei Gravely, 1924

Synonym: Pardosa mackenziei (Gravely, 1924)

Distribution: Pakistan, India, Bangladesh

Comments: Endemic to South Asia. Transfer of

Lycosa mackenziei to genus Pardosa was considered

incorrect and thus, original name was reinstated by

Tikader and Malhotra (1980).

VI.b. Genus Pardosa C. L. Koch, 1847

1. Pardosa algoides Schenkel, 1963

Synonyms: Pardosa uncata Schenkel, 1963

Pardosa ladakhensis Tikader, 1977

Pardosa ehrenfriedi Brignoli, 1983

Distribution: India, Bangladesh, China

Comments: Pardosa ladakhensis was synonymised with Pardosa algoides by Yu and Song (1988). Bangladesh is recent addition in distribution of this species.
2. Pardosa minuta Tikader \& Malhotra, 1976

Synonym: Pardosa minutus Tikader \& Malhotra, 1976 Distribution: India, Bangladesh

Comments: Endemic to South Asia. Bangladesh is recent addition in distribution of this species.

3. Pardosa oakleyi Gravely, 1924

Distribution: Pakistan, India, Bangladesh

Comments: Endemic to South Asia

4. Pardosa royi Biswas \& Raychaudhuri, 2003

Comments: Endemic to Bangladesh

5. Pardosa shyamae (Tikader, 1970)

Synonym: Lycosa shyamae Tikader, 1970

Distribution: India, Bangladesh, China

Comments: Lycosa shyamae was transferred to genus Pardosa by Tikader and Malhotra (1980). Bangladesh is recent addition in distribution of this species.

6. Pardosa songosa Tikader \& Malhotra, 1976

Distribution: India, Bangladesh, China

Comments: Bangladesh is recent addition in distribution of this species.

\section{FAMILY MITURGIDAE SIMON, 1885}

VII.a. Genus Cheiracanthium C. L. Koch, 1839

Comments: Genus Cheiracanthium (Clubionidae) was transferred to family Miturgidae by Bonaldo \& Brescovit (1997).

1. Cheiracanthium melanostomum (Thorell, 1895)

Synonyms: Eutittha melanostoma Thorell, 1895

Cheiracathium sadanai Tikader, 1976

Cheiracanthium melanostoma (Thorell, 1895)

Distribution: India, Bangladesh, Myanmar

Comments: Cheiracathium sadanai was synonymised with Cheiracanthium melanostomum by DeelemanReinhold (2001)

2. Cheiracanthium mysorense Majumder \& Tikader, 1991 Synonym: Cheiracanthium mysorensis Majumder \& Tikader, 1991

Distribution: Bangladesh, India

Comments: Endemic to South Asia. This species is recently reported from Bangladesh.

3. Cheiracanthium sikkimense Majumder \& Tikader, 1991 Synonym: Cheiracanthium sikkimensis Majumder \& Tikader, 1991

Distribution: Bangladesh, India

Comments: Endemic to South Asia. This species is recently reported from Bangladesh.

4. Cheiracanthium tagorei Biswas \& Raychaudhuri, 2003 Comments: Endemic to Bangladesh

VIII. FAMILY PHILODROMIDAE THORELL, 1870

VIII.a. Genus Tibellus Simon, 1875

Comments: Genus Tibellus was removed from family

Thomisidae and placed in the family Philodromidae by Homann (1975).

1. Tibellus shikerpurensis Biswas \& Raychaudhuri, 2003 Comments: Endemic to Bangladesh

IX. FAMILY PISAURIDAE SIMON, 1890

IX.a. Genus Hygropoda Thorell, 1894

1. Hygropoda longimana (Stoliczka, 1869)

Synonym:Dolomedes longimanus Stoliczka, 1869

Distribution: Bangladesh, Malaysia

\section{FAMILY SALTICIDAE BLACKWALL, 1841}

X. Genus Marpissa C.L. Koch, 1846

1. Marpissa carinata Butt \& Beg, 2000

Comments: Endemic to Pakistan

X.b. Genus Phidippus C. L. Koch, 1846

1. Phidippus majumderi Biswas, 1999

Comments: Endemic to Bangladesh

X.c. Genus Plexippus C.L. Koch, 1846

1. Plexippus wesolowskae Biswas \& Raychaudhuri, 1998

Synonyms: Plexippus wesolowskai Biswas \& Raychaudhuri, 1998

Comments: Endemic to Bangladesh

2. Plexippus zabkai Biswas, 1999

Comments: Endemic to Bangladesh

X.d. Genus Zeuxippus Thorell, 1891

1. Zeuxippus pallidus Thorell, 1895

Synonyms: Rhene argentata Wesolowska, 1981

Distribution: Bangladesh, Myanmar, China, Vietnam

XI. FAMILY TETRAGNATHIDAE MENGE, 1866

Comments: Genera Leucauge and Meta were originally placed in the family Tetragnathidae which is followed by Platnick (2006) and this paper, Tikader (1987) placed it in the family Araneidae without any explanation.

XI.a. Genus Dyschiriognatha Simon, 1893

1. Dyschiriognatha dentata Zhu \& Wen, 1978

Synonyms: Dyschiriognatha hawigtenera Barrion \& Litsinger, 1995

Distribution: Bangladesh to China, Japan, Philippines Comments: Dyschiriognatha hawigtenera was synonymised with Dyschiriognatha dentata by Zhu, Song \& Zhang (2003).

XI.b. Genus Tetragnatha Latreille, 1804

Synonym: Genus Eucta Simon, 1881

Comments: Genus Eucta was synonymised with Tetragnatha by Levi (1981).

1. Tetragnatha hasselti Thorell, 1890 Synonym: Tetragnatha hasseltii Thorell, 1890 Tetragnatha aduncata Wang, 1991

Distribution: Bangladesh to China, Sulawesi Comments: According to Platnick (2006), Tetragnatha aduncata was synonymised with Tetragnatha hasselti.

2. Tetragnatha khanjahaniBiswas \& Raychaudhuri, 1996 Comments: Endemic to Bangladesh

3. Tetragnatha mandibulata Walckenaer, 1842 Synonyms: Tetragnatha minax Blackwall, 1877 Tetragnatha minatoria Simon, 1877 Tetragnatha leptognatha Thorell, 1877

Distribution: West Africa, India to Philippines, Australia

4. Tetragnatha maxillosa Thorell, 1895 Synonyms: Tetragnatha japonica Bösenberg \& Strand, 1906

Tetragnatha maxillosa insignita Strand, 1911

Tetragnatha listeri Gravely, 1921

Tetragnatha conformans Chamberlin, 1924

Tetragnatha japonica (Bösenberg \& Strand, 1906)

Tetragnatha propioides Schenkel, 1936

Tetragnatha cliens Yin, 1976

Tetragnatha diensens Zhao, 1993

Distribution: South Africa, Bangladesh to Philippines,

New Hebrides

5. Tetragnatha virescens Okuma, 1979

Distribution: Bangladesh, Sri Lanka to Indonesia, 
Philippines

XII. FAMILY THERAPHOSIDAE THORELL, 1870

XII.a. Genus Chilobrachys Karsch, 1891

1. Chilobrachys andersoni (Pocock, 1895)

Synonyms: Musagetes andersonii Pocock, 1895

Phlogius cervinus Thorell, 1895

Musagetes rufofuscus Thorell, 1897

Distribution: Bangladesh, Myanmar, Malaysia

2. Chilobrachys stridulans (Wood Mason, 1877)

Synonyms: Mygale stridulans Wood-Mason, 1877

Musagetes masoni Pocock, 1895

Chilobrachys masoni (Pocock, 1895)

Distribution: India, Bangladesh

Comments: Endemic to South Asia. According to

Platnick (2006) Chilobrachys masoni was

synonymised with Chilobrachys stridulans.

\section{FAMILY THOMISIDAE SUNDEVALL, 1833}

XIII.a. Genus Runcinia Simon, 1875

1. Runcinia acuminata (Thorell, 1881)

Synonyms: Misumena elongata L. Koch, 1874

Pistius acuminatus Thorell, 1881

Runcinia elongata (L. Koch, 1874)

Runcinia albostriata Hu, 1984

Distribution: Bangladesh to Japan, New Guinea, Australia

2. Runcinia affinis Simon, 1897

Synonyms: Runcinia annamita Simon, 1903

Runcinia albostriata Bösenberg \& Strand, 1906

Plancinus advecticius Simon, 1909

Runcinia cataracta Lawrence, 1927

Thomisus cherapunjeus Tikader, 1966

Runcinia chauhani Sen \& Basu, 1972

Distribution: Africa, Bangladesh, India to Japan,

Philippines, Java

Comments: Thomisus cherapunjeus and Runcinia chauhani were synonymised with Runcinia affinis by Lehtinen (2005).

Bangladesh spider summary

Number of families: 13

Number of Genera: 24

Number of Species: 50

Number of subspecies: 0

Number of Endemic Families: 0

Number of Endemic Genera: 0

Number of Endemic Species: 18

\section{Checklist 3. Spiders of Bhutan}

I. FAMILY AGELENIDAE C.L. KOCH, 1837

I.a. Genus Tegenaria Latreille, 1804

1. Tegenaria wittmeri Brignoli, 1978

Comments: Endemic to Bhutan

\section{FAMILY AMAUROBIIDAE THORELL, 1870}

II.a. Genus Draconarius Ovtchinnikov, 1999 1. Draconarius baronii (Brignoli, 1978)

Synonyms: Coelotes baronii Brignoli, 1978

Comments: Endemic to Bhutan. Wang (2002) transferred male of Coelotes baronii to genus Draconarius and female of Coelotes baronii was transferred to genus Himalcoelotes.

2. Draconarius schenkeli (Brignoli, 1978)

Synonyms: Coelotes schenkeli Brignoli, 1978

Comments: Endemic to Bhutan. Coelotes schenke was transferred to genus Draconarius by Wang (2002).

3. Draconarius stemmleri (Brignoli, 1978) Synonyms: Coelotes stemmleri Brignoli, 1978 Comments: Endemic to Bhutan. Coelotes stemmleri was transferred to genus Draconarius by Wang (2002) .

4. Draconarius wuermlii (Brignoli, 1978)

Synonyms: Coelotes wuermlii Brignoli, 1978

Paracoelotes wuermlii Brignoli, 1982

Comments: Endemic to Bhutan. Wang (2002)

transferred Paracoelotes wuermlii to genus Draconarius.

II.b. Genus Himalcoelotes Wang, 2002

1. Himalcoelotes brignolii Wang, 2002

Synonyms: Coelotes baronii Brignoli, 1978

Comments: Endemic to Bhutan. Female of Coelotes

baronii was transferred to genus Himalcoelotes brignoli by Wang (2002).

\section{FAMILY ARANEIDAE SIMON, 1895}

III.a. Genus Cyclosa Menge, 1866

1. Cyclosa quinqueguttata (Thorell, 1881)

Synonyms: Epeira quinque-guttata Thorell, 1881

Epeira hybophora Thorell, 1887

Cyclosa hybophora (Thorell, 1887)

Cyclosa fissicauda Simon, 1889

Distribution: India, Bhutan, Myanmar, China, Taiwan

Comments: Cyclosa hybophora was synonymised

with Cyclosa quinqueguttata by Roberts (1983)

Cyclosa fissicauda was synonymised with Cyclosa

quinqueguttata by Tanikawa \& Ono (1993).

III.b. Genus Neoscona Simon, 1864

1. Neoscona chrysanthusi Tikader \& Bal, 1981

Distribution: Bhutan, India

Comments: Endemic to South Asia

IV. FAMILY HAHNIIDAE BERTKAU, 1878

IV.a. Genus Hahnia C.L. Koch, 1841

1. Hahnia caelebs Brignoli, 1978

Comments: Endemic to Bhutan

2. Hahnia innupta Brignoli, 1978

Comments: Endemic to Bhutan

3. Hahnia lehtineni Brignoli, 1978

Comments: Endemic to Bhutan

4. Hahnia musica Brignoli, 1978

Comments: Endemic to Bhutan

5. Hahnia tikaderi Brignoli, 1978

Comments: Endemic to Bhutan
V. FAMILY LINYPHIIDAE BLACKWALL, 1859

V.a. Genus Heterolinyphia Wunderlich, 1973

Comments: This genus is endemic to South Asia.

1. Heterolinyphia secunda Thaler, 1999

Comments: Endemic to Bhutan

\section{FAMILY LYCOSIDAE SUNDEVALL, 1833}

VI.a. Genus Dorjulopirata Buchar, 1997

Comments: This is a monotypic genus and endemic to Bhutan

1. Dorjulopirata dorjulanus Buchar, 1997

Synonym: Dorjulopirata dorjulana Buchar, 1997

Comments: Endemic to Bhutan

VI.b. Genus Hippasa Simon, 1885

1. Hippasa bifasciata Buchar, 1997

Comments: Endemic to Bhutan

VI.c. Genus Hogna Simon, 1885

Synonym: Genus Lycorma Simon, 1885

Comments: The genus Lycorma was synonymised

with the genus Hogna by Wunderlich (1992).

1. Hogna himalayensis (Gravely, 1924)

Synonyms: Lycosa himalayensis Gravely, 1924

Lycorma himalayensis Buchar, 1997

Distribution: India, Bhutan, China

Comments: Lycosa himalayensis was transferred to genus Hogna by Buchar (1997).

VI.d. Genus Lycosa Latreille, 1804

Synonym: Genus Allohogna Roewer, 1955

Comments: Allohogna was synonymised with genus

Lycosa by Fuhn \& Niculescu-Burlacu (1971).

1. Lycosa bistriata Gravely, 1924

Synonym: Hogna bistriata (Gravely, 1924)

Distribution: India, Bhutan

Comments: Endemic to South Asia. Transfer of

Lycosa bistriata to genus Hogna was considered incorrect and thus, original name was reinstated by Tikader and Malhotra (1980).

2. Lycosa kempi Gravely, 1924

Synonym: Piratula kempi (Gravely, 1924)

Distribution: India, Pakistan, Bhutan, China

Comments: Transfer of Lycosa kempi to genus

Piratula was considered incorrect and thus the original name was reinstated by Tikader (1970).

3. Lycosa nigrotibialis Simon, 1884

Synonyms: Hogna nigrotibialis (Simon, 1884)

Allocosa nigrotibialis (Simon, 1884)

Distribution: India, Bhutan, Myanma

Comments: Transfer of Lycosa nigrotibialis to genus

Hogna and later to genus Allocosa was considered incorrect and thus the original name was reinstated by Tikader \& Malhotra (1980).

VI.e. Genus Trochosa C.L. Koch, 1847

1. Trochosa dentichelis Buchar, 1997

Comments: Endemic to Bhutan

VI.f. Genus Zoica Simon, 1898

Comments: Considered a senior synonym of genus Flanona Simon, 1898 by Lehtinen \& Hippa (1979).

1. Zoica oculata Buchar, 1997

Comments: Endemic to Bhutan

VII. FAMILY MIMETIDAE SIMON, 1881

VII.a. Genus Ero C. L. Koch, 1836

1. Ero cachinnans Brignoli, 1978

Comments: Endemic to Bhutan 


\section{FAMILY NEPHILIDAE SIMON, 1894}

Comments: Subfamily Nephilinae under family

Tetragnathidae was elevated to family level by Kuntner (2006). Genera Clitaetra, Herennia, Nephila and Nephilengys were transferred from family Tetragnathidae to family Nephilidae.

VIII.a. Genus Nephila Leach, 1815

1. Nephila clavata L. Koch, 1878

Synonyms: Nephila limbata Thorell, 1898

Nephila obnubila Simon, 1906

Distribution: Bhutan, Pakistan, India to Japan

\section{FAMILY OONOPIDAE SIMON, 1890}

IX.a. Genus Camptoscaphiella Caporiacco, 1934

1. Camptoscaphiella hilaris Brignoli, 1978

Comments: Endemic to Bhutan

IX.b. Genus Epectris Simon, 1893

1. Epectris aenobarbus Brignoli, 1978

Comments: Endemic to Bhutan

IX.c. Genus Ischnothyreus Simon, 1893

1. Ischnothyreus shillongensis Tikader, 1968

Synonym: Camptoscaphiella shillongensis Brignoli, 1976

Distribution: India, Bhutan

Comments: Endemic to South Asia. Transfer of

Ischnothyreus shillongensis to genus

Camptoscaphiella Caporiacco, 1934 was considered

incorrect and thus, original name was reinstated by Brignoli (1978).

IX.d. Genus Opopaea Simon, 1891

1. Opopaea sponsa Brignoli, 1978

Comments: Endemic to Bhutan

IX.e. Genus Orchestina Simon, 1882

1. Orchestina aerumnae Brignoli, 1978

Comments: Endemic to Bhutan

\section{FAMILY SALTICIDAE BLACKWALL, 1841}

X.a. Genus Bianor Peckham \& Peckham, 1886

1. Bianor pseudomaculatus Logunov, 2001

Synonym: Bianor maculatus Zabka, 1985

Distribution: India, Bhutan, Vietnam

Comments: According to Logunov (2001), Bianor

maculatus Zabka, 1985 is a misidentification.

X.b. Genus Carrhotus Thorell, 1891

1. Carrhotus kamjeensis Jastrzebski, 1999

Comments: Endemic to Bhutan

X.c. Carrhotus samchiensis Jastrzebski, 1999

Comments: Endemic to Bhutan

X.d. Genus Evarcha Simon, 1902

1. Evarcha pococki Zabka, 1985

Distribution: Bhutan to Vietnam, China

X.e. Genus Harmochirus Simon, 1885

1. Harmochirus brachiatus (Thorell, 1977)

Synonyms: Ballus brachiatus Thorell, 1877

Harmochirus malaccensis Simon, 1885

Harmochirus nervosus Thorell, 1890

Harmochirus insulanus Namkung, 2002

Distribution: India, Bhutan to Taiwan, Indonesia

X.f. Genus Helicius Zabka, 1981

1. Helicius hillaryi Zabka, 1981

Comments: Endemic to Bhutan

X.g. Genus Heliophanoides Prószyn'ski, 1992

Comments: Endemic to South Asia.

1. Heliophanoides bhutanicus Prószyn'ski, 1992

Comments: Endemic to Bhutan
X.h. Genus Langona Simon, 1901

1. Langona bhutanica Prószyn'ski, 1978

Distribution: Bhutan, China

X.i. Genus Neobrettus Wanless, 1984

1. Neobrettus tibialis (Prószyn'ski, 1978)

Synonym: Cyrba tibialis Prószyn'ski, 1978

Distribution: Bhutan to Malaysia, Borneo

Comments: Cyrba tibialis was transferred to genus

Neobrettus by Wanless (1984).

X.j. Genus Pancorius Simon, 1902

1. Pancorius changricus Zabka, 1990

Comments: Endemic to Bhutan

2. Pancorius magniformis Zabka, 1990

Comments: Endemic to Bhutan

3. Pancorius wangdicus Zabka, 1990

Comments: Endemic to Bhutan

X.k. Genus Phintella Strand, 1906

1. Phintella volupe (Karsch, 1879)

Synonym: Attus volupe Karsch, 1879

Distribution: Sri Lanka, Bhutan

Comments: Endemic to South Asia. Attus volupe was transferred to genus Phintella by Zabka (1988).

X.I. Genus Phlegra Simon, 1876

1. Phlegra particeps (O. P.-Cambridge, 1872)

Synonym: Salticus particeps O. P.-Cambridge, 1872

Distribution: Israel to Bhutan

Comments: Salticus particeps was transferred to genus Phlegra by Simon (1876).

2. Phlegra samchiensis Prószyn'ski, 1978

Comments: Endemic to Bhutan

3. Phlegra thibetana Simon, 1901

Distribution: Bhutan, China

X.m. Genus Plexippoides Prószyn'ski, 1984 1. Plexippoides dilucidus Próchniewicz, 1990

Comments: Endemic to Bhutan

X.n. Genus Plexippus C.L. Koch, 1846

1. Plexippus bhutani Zabka, 1990

Distribution: Bhutan, China

X.o. Genus Pseudamycus Simon, 1885

1. Pseudamycus bhutani Zabka, 1990

Comments: Endemic to Bhutan

X.p. Genus Rafalus Prószyn'ski, 1999

1. Rafalus wittmeri (Prószyn'ski, 1978)

Synonym: Aelurillus wittmeri Prószyn'ski, 1978

Comments: Endemic to Bhutan. Aelurillus wittmer

was transferred to genus Rafalus by Prószyn'ski (1999).

X.q. Genus Rhene Thorell, 1869

1. Rhene flavicomans Simon, 1902

Distribution: India, Bhutan, Sri Lanka

Comments: Endemic to South Asia

2. Rhene phuntsholingensis Jastrzebski, 1997

Distribution: Bhutan, Nepal

Comments: Endemic to South Asia

X.r. Genus Synagelides Strand, 1906

1. Synagelides wangdicus Bohdanowicz, 1978

Comments: Endemic to Bhutan

2. Synagelides wuermlii Bohdanowicz, 1978

Comments: Endemic to Bhutan

X.s. Genus Telamonia Thorell, 1887

1. Telamonia dimidiata (Simon, 1899)
Synonyms: Viciria dimidiata Simon, 1899

Phidippus pateli Tikader, 1974

Distribution: India, Bhutan, Sumatra

Comments: Phidippus pateli was synonymised with

Telamonia dimidiata by Prószyn'ski (1992); Viciria

dimidiata was transferred to genus Telamonia by

Prószyn'ski (1984b).

2. Telamonia dissimilis Próchniewicz, 1990

Comments: Endemic to Bhutan

3. Telamonia laecta Próchniewicz, 1990

Comments: Endemic to Bhutan

4. Telamonia prima Próchniewicz, 1990

Comments: Endemic to Bhutan

X.t. Genus Yaginumaella Prószyn'ski, 1979

1. Yaginumaella bhutanica Zabka, 1981

Comments: Endemic to Bhutan

2. Yaginumaella cambridgei Zabka, 1981

Comments: Endemic to Bhutan

3. Yaginumaella gogonaica Zabka, 1981

Comments: Endemic to Bhutan

4. Yaginumaella helvetorum Zabka, 1981

Comments: Endemic to Bhutan

5. Yaginumaella hybrida Zabka, 1981

Comments: Endemic to Bhutan

6. Yaginumaella incognita Zabka, 1981

Comments: Endemic to Bhutan

7. Yaginumaella intermedia Zabka, 1981

Comments: Endemic to Bhutan

8. Yaginumaella montana Zabka, 1981

Distribution: China, Bhutan

9. Yaginumaella nobilis Zabka, 1981

Comments: Endemic to Bhutan

10. Yaginumaella nova Zabka, 1981

Comments: Endemic to Bhutan

11. Yaginumaella orientalis Zabka, 1981

Comments: Endemic to Bhutan

12. Yaginumaella pilosa Zabka, 1981

Comments: Endemic to Bhutan

13. Yaginumaella silvatica Zabka, 1981

Comments: Endemic to Bhutan

14. Yaginumaella simoni Zabka, 1981

Comments: Endemic to Bhutan

15. Yaginumaella stemmleri Zabka, 1981

Comments: Endemic to Bhutan

16. Yaginumaella strandi Zabka, 1981

Comments: Endemic to Bhutan

17. Yaginumaella supina Zabka, 1981

Comments: Endemic to Bhutan

18. Yaginumaella tenella Zabka, 1981

Comments: Endemic to Bhutan

19. Yaginumaella thimphuica Zabka, 1981

Comments: Endemic to Bhutan

20. Yaginumaella urbanii Zabka, 1981

Comments: Endemic to Bhutan 
21. Yaginumaella versicolor Zabka, 1981 Comments: Endemic to Bhutan

22. Yaginumaella wangdica Zabka, 1981 Comments: Endemic to Bhutan

23. Yaginumaella wuermli Zabka, 1981 Synonym: Ptocasius wuermli Hu, 2001 Distribution: Bhutan, China

XI. FAMILY SPARASSIDAE BERTKAU, 1872 Comments: Considered senior synonym of Comments: Endemic to Bhutan Heteropodidae by Jäger (1999).

XI.a. Genus Bhutaniella Jäger, 2000

1. Bhutaniella dunlopi Jäger, 2001

Comments: Endemic to Bhutan

2. Bhutaniella gruberi Jäger, 2001 Comments: Endemic to Bhutan

3. Bhutaniella haenggii Jäger, 2001

Comments: Endemic to Bhutan

XI.b. Genus Pseudopoda Jäger, 2000

1. Pseudopoda albonotata Jäger, 2001

Comments: Endemic to Bhutan

2. Pseudopoda gogona Jäger, 2001

Comments: Endemic to Bhutan

XII. FAMILY THOMISIDAE SUNDEVALL, 1833

XII.a. Genus Ebrechtella Dahl, 1907

1. Ebrechtella pseudovatia (Schenkel, 1936)

Synonym: Misumena pseudovatia Schenkel, 1936

Misumenops pseudovatius (Schenkel, 1936)

Misumenops wenensis Zhu, Song \& Tang, in Tang,

Song \& Zhu, 1995

Distribution: Bhutan, China

Comments: Misumenops pseudovatius was transferred to the genus Ebrechtella by Lehtinen (2005).

XII.b. Genus Loxobates Thorell, 1877

1. Loxobates minor Ono, 2001

Comments: Endemic to Bhutan

XII.c. Genus Lysiteles Simon, 1895

1. Lysiteles ambrosii Ono, 2001

Comments: Endemic to Bhutan

2. Lysiteles amoenus Ono, 1980

Distribution: Bhutan, Taiwan

3. Lysiteles bhutanus Ono, 2001

Comments: Endemic to Bhutan

4. Lysiteles himalayensis Ono, 1979

Distribution: Nepal, Bhutan

Comments: Endemic to South Asia

5. Lysiteles kunmingensis Song \& Zhao, 1994

Distribution: Bhutan, China

6. Lysiteles minusculus Song \& Chai, 1990

Distribution: Bhutan, China

7. Lysiteles niger Ono, 1979

Distribution: Bhutan, Nepal

Comments: Endemic to South Asia

8. Lysiteles punctiger Ono, 2001

Comments: Endemic to Bhutan

9. Lysiteles saltus Ono, 1979

Synonym: Xysticus himalayaensis $\mathrm{Hu} \& \mathrm{Li}, 1987$
Xysticus mandali $\mathrm{Hu} \& \mathrm{Li}, 1987$

Distribution: Nepal, Bhutan, China

10. Lysiteles wittmeri Ono, 2001

Comments: Endemic to Bhutan

XII.d. Genus Oxytate L. Koch, 1878 Synonym: Genus Dieta Simon, 1880

Comments: Genus Dieta was synonymised with genus Oxytate by Song et al. (1982).

1. Oxytate bhutanica Ono, 2001

XII.e. Genus Philodamia Thorell, 1894

1. Philodamia armillata Thorell, 1895

Distribution: Bhutan, Myanmar

XII.f. Genus Stiphropus Gerstäcker, 1873

1. Stiphropus soureni Sen, 1964

Distribution: India, Nepal, Bhutan

Comments: Endemic to South Asia

XII.g. Genus Strigoplus Simon, 1885

1. Strigoplus albostriatus Simon, 1885

Synonym: Peltorrhynchus rostratus Thorell, 1892

Distribution: Bhutan, Malaysia, Java

XII.h. Genus Synema Simon, 1864

1. Synema albomaculatum Ono, 2001

Comments: Endemic to Bhutan

XII.i. Genus Talaus Simon, 1886

1. Talaus samchiOno, 2001

Comments: Endemic to Bhutan

XII.j. Genus Xysticus C.L. Koch, 1835

1. Xysticus croceus Fox, 1937

Synonyms: Xysticus ephippiatus Bösenberg \& Strand 1906

Xysticus sujatai Tikader, 1962

Distribution: India, Nepal, Bhutan, China, Korea,

Japan

Comments: Xysticus sujatai was synonymised with

Xysticus croceus by Ono (1988).

2. Xysticus simplicipalpatus Ono, 1978

Distribution: Nepal, Bhutan

Comments: Endemic to South Asia

\section{Bhutan spider summary}

Number of Families: 12

Number of Genera: 51

Number of Species: 105

Number of Subspecies: 0

Number of Endemic Families: 0

Number of Endemic Genera: 1

Number of Endemic Species: 71

\section{Checklist 4. Spiders of India (Web supplement)}

\section{India spider summary}

Number of Families: 60

Number of Genera: 365

Number of Species: 1447

Number of Subspecies: 9

Number of Endemic Families: 0

Number of Endemic Genera: 19

Number of Endemic Species: 1053 included by Platnick (2006) as occurring in Pakistan superseding Siliwal et al. (2005) and thus these species are South Asian endemic rather than Indian endemics.

Due to page constraints and printing costs, the 2007 updated checklist of Indian spiders is available in the web supplement of this paper at www.zoosprint.org.

\section{Maldives}

In Maldives, only two non-endemic species of spiders belonging to two genera and two families have been reported (Checklist 5). Argiope anasuja Thorell, 1887 and Tetragnatha foveata Karsch, 1891 are South Asian endemics and are also reported from India, Pakistan and Sri Lanka.

\section{Nepal}

In Nepal, 222 species of spider in 79 genera and 23 families have been reported (Checklist 6). Four of 79 genera are endemic to the country and of which only single genus is monotypic. Of these 222 species, 176 are endemic to the country (Table 2). Studies on spiders were initiated in mid 20th century and major contribution was done by Jäger (2000, 2001), who described 32 species, Ono (1978, 1979, 1983) described 19 species, Bohdanowicz (1979, 1987) described 14 species and Brignoli (1972, 1973, 1976, $1978,1983)$ described 12 species.

\section{Pakistan}

In Pakistan, 138 species of spider in 79 genera and 22 families have been reported (Checklist 7 ). The genus Sivalicus is monotypic and endemic to the country. Out of 138 species, 72 are endemic to the country (Table 2). Major contribution to spider study was done by Dyal (1935, 1957) by describing 42 species. The publications before independence and partition of India and Pakistan (1947) indicate India and Platnick (2006) has retained India in distribution of many spider species whose type locality and known distribution is in present Pakistan.

\section{Sri Lanka}

In Sri Lanka, 354 species of spider in 213 genera and 45 families have been reported (Checklist 8). Twenty-two 


\section{Checklist 5. Spiders of Maldives}

\section{FAMILY ARANEIDAE SIMON, 1895}

I.a. Genus Argiope Audouin, 1826

1. Argiope anasuja Thorell, 1887

Synonyms: Argiope anasuja fletcheri Hirst, 1911

Argiope plagiata Karsch, 1891

Distribution: India, Maldives, Pakistan

Comments: Endemic to South Asia

II. FAMILY TETRAGNATHIDAE MENGE, 1866 II.a. Genus Tetragnatha Latreille, 1804

Synonym: Genus Eucta Simon, 1881

Comments: Genus Eucta was synonymised with

Tetragnatha by Levi (1981).

1. Tetragnatha foveata Karsch, 1891

Distribution: India, Sri Lanka, Maldives

Comments: Endemic to South Asia

Maldives spider summary

Number of Families: 2

Number of Genera: 2

Number of Species: 2

Number of Subspecies:

Number of Endemic Families: 0

Number of Endemic Genera: 0

Number of Endemic Species:

\section{Checklist 6. Spiders of Nepal}

I. FAMILY AGELENIDAE C.L. KOCH, 1837

I.a. Genus Agelena Walckenaer, 1805

1. Agelena lukla Nishikawa, 1980

Comments: Endemic to Nepal

2. Agelena sherpa Nishikawa, 1980

Comments: Endemic to Nepal

I.b. Genus Tegenaria Latreille, 1804

1. Tegenaria lunakensis Tikader, 1964

Comments: Endemic to Nepal

\section{FAMILY AMAUROBIIDAE THORELL, 1870}

II.a. Genus Amaurobius C.L. Koch, 1837

1. Amaurobius milloti Hubert, 1973

Comments: Endemic to Nepa

II.b. Genus Draconarius Ovtchinnikov, 1999

1. Draconarius gurkha (Brignoli, 1976)

Synonyms: Coelotes gurkha Brignoli, 1976

Coelotes lama Brignoli, 1976

Comments: Endemic to Nepal. Wang (2002) V.c. Genus Phaeocedus Simon, 1893

transferred Coelotes gurkha to genus Draconarius 1. Phaeocedus mosambaensis Tikader, 1964

and synonymised Coelotes lama with Coelotes Comments: Endemic to Nepal

gurkha.

II.c. Genus Himalcoelotes Wang, 2002

1. Himalcoelotes aequoreus Wang, 2002

Comments: Endemic to Nepal

2. Himalcoelotes bursarius Wang, 2002

Comments: Endemic to Nepal

3. Himalcoelotes diatropos Wang, 2002

Comments: Endemic to Nepal

4. Himalcoelotes gyirongensis ( $\mathrm{Hu} \& \mathrm{Li}, 1987)$

Synonyms: Coelotes gyirongensis $\mathrm{Hu} \& \mathrm{Li}, 1987$

Distribution: Nepal, China

Comments: Coelotes gyirongensis was transferred to genus Himalcoelotes by Wang (2002).

5. Himalcoelotes martensi Wang, 2002

Comments: Endemic to Nepal

6. Himalcoelotes pirum Wang, 2002

Comments: Endemic to Nepal

7. Himalcoelotes sherpa (Brignoli, 1976)

Synonyms: Coelotes sherpa Brignoli, 1976

Comments: Endemic to Nepal. Coelotes sherpa was

transferred to genus Himalcoelotes by Wang (2002).

8. Himalcoelotes subsherpa Wang, 2002

Comments: Endemic to Nepal

9. Himalcoelotes syntomos Wang, 2002

Comments: Endemic to Nepal

\section{FAMILY ANAPIDAE SIMON, 1895}

III.a. Genus Metanapis Brignoli, 198

1. Metanapis montisemodi (Brignoli, 1978)

Synonyms: Pseudanapis montisemodi Brignoli, 1978

Comments: Endemic to Nepal. Brignoli (1891)

transferred Pseudanapis montisemodi to genus Metanapis.

2. Metanapis tectimundi (Brignoli, 1978)

Synonyms: Pseudanapis tectimundi Brignoli, 1978।

Comments: Endemic to Nepal. Brignoli (1891)

transferred Pseudanapis tectimundi to genus

Metanapis.
IV. FAMILY ERESIDAE C.L. KOCH, 1851

IV.a. Genus Stegodyphus Simon, 1873

1. Stegodyphus sarasinorum Karsch, 1891

Distribution: India, Sri Lanka, Nepal

Comments: Endemic to South Asia

\section{FAMILY GNAPHOSIDAE POCOCK, 1898}

V.a. Genus Drassodes Westring, 1851

1. Drassodes phagduaensis Tikader, 1964

Comments: Endemic to Nepal

V.b. Genus Gnaphosa Latreille, 1804

1. Gnaphosa mandschurica Schenkel, 1963

Synonym: Gnaphosa glandifera Schenkel, 1963

Gnaphosa holmi Schenkel, 1963

Gnaphosa charitonowi Schenkel, 1963

Gnaphosa braendegaardi Schenkel, 1963

Gnaphosa berlandi Schenkel, 1963

Distribution: Russia, Mongolia, China, Nepal

2. Gnaphosa moerens O. P.-Cambridge, 1885

Distribution: China, Nepa

\section{FAMILY HAHNIIDAE BERTKAU, 1878}

VI.a. Genus Hahnia C.L. Koch, 1841

1. Hahnia alini Tikader, 1964

Comments: Endemic to Nepal

VI.b. Genus Neoantistea Gertsch, 1934

1. Neoantistea janetscheki Brignoli, 1976

Comments: Endemic to Nepal

VII. FAMILY HERSILIIDAE THORELL, 1870

VII.a. Genus Hersilia Audouin, 1826

1. Hersilia martensi Baehr \& Baehr, 1993

Comments: Endemic to Nepal

2. Hersilia nepalensis Baehr \& Baehr, 1993

Comments: Endemic to Nepal

VIII. FAMILY LINYPHIIDAE BLACKWALL, 1859

VIII.a. Genus Agyneta Hull, 1911

1. Agyneta bueko Wunderlich, 1983

Comments: Endemic to Nepal

2. Agyneta jiriensis Wunderlich, 1983

Comments: Endemic to Nepal

3. Agyneta muriensis Wunderlich, 1983

Comments: Endemic to Nepal

4. Agyneta yulungiensis Wunderlich, 1983

Comments: Endemic to Nepal

VIII.b. Genus Asthenargus Simon \& Fage, 1922

1. Arachosinella thaleri Wunderlich, 1983

Comments: Endemic to Nepal

VIII.c. Genus Caviphantes Oi, 1960

1. Caviphantes pseudosaxetorum Wunderlich, 1979

Distribution: Nepal, China, Japan

VIII.d. Genus Erigone Audouin, 1826

1. Erigone nepalensis Wunderlich, 1983

Comments: Endemic to Nepal

2. Erigone ourania Crosby \& Bishop, 1928

Distribution: Nepal to China 
VIII.e. Genus Gongylidiellum Simon, 1884 1. Gongylidiellum kathmanduense Wunderlich, 1983 Comments: Endemic to Nepal

2. Gongylidiellum nepalense Wunderlich, 1983 Comments: Endemic to Nepal

VIII.f. Genus Gorbothorax Tanasevitch, 1998 Comments: This genus is endemic to Nepal.

1. Gorbothorax comatus Tanasevitch, 1998 Comments: Endemic to Nepal

2. Gorbothorax conicus Tanasevitch, 1998 Comments: Endemic to Nepal

3. Gorbothorax setifer Tanasevitch, 1998 Comments: Endemic to Nepal

4. Gorbothorax ungibbus Tanasevitch, 1998 Comments: Endemic to Nepal

5. Gorbothorax wunderlichi (Brignoli, 1983) Synonym: Oedothorax maculatus Wunderlich, 1974 Oedothorax wunderlichi Brignoli, 1983

Comments: Endemic to Nepal. Oedothorax 5. Lepthyphantes bifurcatoides Tanasevitch, 1987 wunderlichi was a replacement name provided by by Comments: Endemic to Nepal

Brignoli (1983) for Oedothorax maculatus as the

species name was preoccupied by Crosby (1905). 6. Lepthyphantes bifurcatus Tanasevitch, 1987

Later this species was trasferred to genus Comments: Endemic to Nepal

Gorbothorax by Tanasevitch (1998).

VIII.g. Genus Helsdingenia Saaristo \& Tanasevitch, 2003 1. Helsdingenia ceylonica (van Helsdingen, 1985)

Distribution: Nepal, Sri Lanka

Comments: Endemic to South Asia

VIII.h. Genus Heterolinyphia Wunderlich, 1973 Comments: This genus is endemic to South Asia.

1. Heterolinyphia tarakotensis Wunderlich, 1973 Distribution: India, Nepal

Comments: Endemic to South Asia

VIII.i. Genus Hilaira Simon, 1884

1. Hilaira dapaensis Wunderlich, 1983

Comments: Endemic to Nepal

VIII.j. Genus Himalaphantes Tanasevitch, 1992

1. Himalaphantes grandiculus (Tanasevitch, 1987)

Synonym: Lepthyphantes grandiculus Tanasevitch, 1987

Comments: Endemic to Nepal. Lepthyphantes

grandiculus was transferred to genus

Himalaphantes by Tanasevitch (1992).

2. Himalaphantes magnus (Tanasevitch, 1987)

Synonym: Lepthyphantes magnus Tanasevitch, 1987

Comments: Endemic to Nepal. Lepthyphantes

magnus was transferred to genus Himalaphantes by

Tanasevitch (1992).

3. Himalaphantes martensi (Thaler, 1987)

Synonym: Lepthyphantes martensi Thaler, 1987

Distribution: India, Nepal

Comments: Endemic to South Asia. Lepthyphantes martensi was transferred to genus Himalaphantes by Tanasevitch (1992).

VIII.k. Genus Hubertella Platnick, 1989 Comment: This genus is endemic to Nepal. Hubertella is a replacement name for genus Hubertia by Platnick (1989) as generic name was preoccupied.

1. Hubertella orientalis (Georgescu, 1977)

Synonym: Hubertia orientalis Georgescu, 1977 1. Meioneta pseudofuscipalpis (Wunderlich, 1983) Comments: Endemic to Nepal
2. Hubertella thankurensis (Wunderlich, 1983)

Synonym: Hubertia thankurensis Wunderlich, 1983 Comments: Endemic to Nepal.

VIII.I. Genus Indophantes Saaristo \& Tanasevitch, 2003 1. Indophantes digitulus (Thaler, 1987) Synonym: Lepthyphantes digitulus Thaler, 1987 tribution: India, Nepal

Comments: Endemic to South Asia. Lepthyphantes Saaristo \& Tanasevitch (2003)

VIII.m. Genus Lepthyphantes Menge, 1866 1. Lepthyphantes alticola Tanasevitch, 1987 Comments: Endemic to Nepal

2. Lepthyphantes anachoretus Tanasevitch, 1987 Comments: Endemic to Nepal

3. Lepthyphantes ancoriformis Tanasevitch, 1987 Comments: Endemic to Nepal

7. Lepthyphantes faustus Tanasevitch, 1987

8. Lepthyphantes nepalensis Tanasevitch, 1987

Comments: Endemic to Nepal

9. Lepthyphantes numilionis Tanasevitch, 1987

Comments: Endemic to Nepal

10. Lepthyphantes occultus Tanasevitch, 1987 Comments: Endemic to Nepal

11. Lepthyphantes rotundatus Tanasevitch, 1987 Comments: Endemic to Nepa

12. Lepthyphantes setifer Tanasevitch, 1987 Comments: Endemic to Nepal

13. Lepthyphantes sherpa Tanasevitch, 1987

Comments: Endemic to Nepal

14. Lepthyphantes theosophicus Tanasevitch, 1987 Comments: Endemic to Nepal

15. Lepthyphantes yeti Tanasevitch, 1987

Comments: Endemic to Nepal

VIII.n. Genus Linyphia Latreille, 1804

1. Linyphia nepalensis Wunderlich, 1983

Comments: Endemic to Nepal

VIII.o. Genus Martensinus Wunderlich, 1973

Comment: This genus is endemic to Nepal.

1. Martensinus annulatus Wunderlich, 1973 Comments: Endemic to Nepal

2. Martensinus micronetiformis Wunderlich, 1973 Comments: Endemic to Nepal

VIII.p. Genus Meioneta Hull, 1920

Comments: Removed from junior synonym of genus Agyneta by Millidge (1977).

Synonym: Agyneta pseudofuscipalpis Wunderlich, 1983
Comments: Endemic to Nepal

VIIII.q. Genus Oedothorax Bertkau, in Förster \& Bertkau, 1883

1. Oedothorax angelus Tanasevitch, 1998

Comments: Endemic to Nepal

2. Oedothorax annulatus Wunderlich, 1974

Comments: Endemic to Nepal

3. Oedothorax asocialis Wunderlich, 1974

Comments: Endemic to Nepal

4. Oedothorax assuetus Tanasevitch, 1998

Comments: Endemic to Nepal

5. Oedothorax clypeellum Tanasevitch, 1998

Comments: Endemic to Nepal

6. Oedothorax coronatus Tanasevitch, 1998

Comments: Endemic to Nepal

7. Oedothorax dismodicoides Wunderlich, 1974

Comments: Endemic to Nepal

8. Oedothorax elongatus Wunderlich, 1974

Comments: Endemic to Nepal

9. Oedothorax falcifer Tanasevitch, 1998

Comments: Endemic to Nepal

10. Oedothorax hirsutus Wunderlich, 1974

Comments: Endemic to Nepal

11. Oedothorax lineatus Wunderlich, 1974

Comments: Endemic to Nepal

12. Oedothorax lucidus Wunderlich, 1974

Comments: Endemic to Nepal

13. Oedothorax malearmatus Tanasevitch, 1998 Comments: Endemic to Nepal

14. Oedothorax modestus Tanasevitch, 1998 Comments: Endemic to Nepal

15. Oedothorax savigniformis Tanasevitch, 1998 Comments: Endemic to Nepal

16. Oedothorax sexoculatus Wunderlich, 1974 Comments: Endemic to Nepa

17. Oedothorax sexoculorum Tanasevitch, 1998 Comments: Endemic to Nepal

18. Oedothorax simplicithorax Tanasevitch, 1998 Comments: Endemic to Nepal

19. Oedothorax tholusus Tanasevitch, 1998

Comments: Endemic to Nepal

20. Oedothorax unicolor Wunderlich, 1974

Comments: Endemic to Nepal

VIII.r. Genus Oia Wunderlich, 1973

1. Oia sororia Wunderlich, 1973

Comments: Endemic to Nepal

VIII.s. Genus Paragongylidiellum Wunderlich, 1973

Comments: It is a monotypic genus and endemic to Nepal.

1. Paragongylidiellum caliginosum Wunderlich, 1973 Comments: Endemic to Nepal

VIII.t. Genus Piniphantes Saaristo \& Tanasevitch, 1996

1. Piniphantes himalayensis (Tanasevitch, 1987) 
Comments: Endemic to Nepal

VIII.u. Genus Porrhomma Simon, 1884 1. Porrhomma marphaense Wunderlich, 1983 Comments: Endemic to Nepal

VIII.v. Genus Saloca Simon, 1926

1. Saloca gorapaniensis Wunderlich, 1983

Synonym: Saloca gorapaniense Wunderlich, 1983

Comments: Endemic to Nepal.

2. Saloca khumbuensis Wunderlich, 1983

Synonym: Saloca khumbuensis Wunderlich, 1983

Comments: Endemic to Nepal.

VIII.w. Genus Tenuiphantes Saaristo \& Tanasevitch, 1996 1. Tenuiphantes plumipes (Tanasevitch, 1987) Synonym: Lepthyphantes plumipes Tanasevitch, 1987 Comments: Endemic to Nepal. Lepthyphantes plumipes was transferred to genus Tenuiphantes by Saaristo \& Tanasevitch (1996).

VIII.x. Genus Walckenaeria Blackwall, 1833 1. Walckenaeria martensi Wunderlich, 1972 Comments: Endemic to Nepal.

2. Walckenaeria nepalensis Wunderlich, 1972 Comments: Endemic to Nepal

\section{FAMILY LYCOSIDAE SUNDEVALL, 1833}

IX.a. Genus Acantholycosa Dahl, 1908 Comments: Buchar \& Thaler (1993) removed this genus from synonym of genus Pardosa.

1. Acantholycosa baltoroi (Caporiacco, 1935) Synonym: Pardosa baltoroi Caporiacco, 1935 Distribution: India, Nepal, China.

IX.b. Genus Arctosa C.L. Koch, 1847 1. Arctosa janetscheki Buchar, 1976 Comments: Endemic to Nepal

2. Arctosa raptor (Kulczyn'ski, 1885)

Synonym: Pirata raptor Kulczyn'ski, 1885

Lycosa quinaria Emerton, 1894

Pirata raptor (Kulczyn'ski, 1885)

Arctosa quinaria (Emerton, 1894)

Trochosa raptor (Kulczyn'ski, 1885)

Distribution: Russia, Nepal, USA, Canada

Comments: Dondale \& Redner (1983) transferred

Trochosa raptor to genus Arctosa and synonymised

Arctosa quinaria with Arctosa raptor.

IX.c. Genus Pardosa C. L. Koch, 1847

1. Pardosa fletcheri (Gravely, 1924)

Synonyms: Lycosa fletcheri Gravely, 1924

Lycosa rothaka Tikader, 1970

Distribution: India, Nepal, Pakistan

Comments: Endemic to South Asia. Lycosa rothaka was synonymised with Pardosa fletcheri by Tikader \& Malhotra (1980)

2. Pardosa martensi Buchar, 1978

Comments: Endemic to Nepal

3. Pardosa mongolica Kulczyn'ski, 1901

Synonym: Lycosa ricta Odenwall, 1901

Lycosa incilis Odenwall, 1901

Pardosa hummeli Schenkel, 1936

Pardosa licenti Schenkel, 1953

Pardosa chaffanjoni Schenkel, 1963

Pardosa tikaderi Buchar, 1984

Pardosa ricta (Odenwall, 1901)

Distribution: Russia, Tajikistan, Nepal, Mongolia, China

4. Pardosa orealis Buchar, 1984

Comments: Endemic to Nepal
5. Pardosa sutherlandi (Gravely, 1924)

Synonyms: Lycosa sutherlandi Gravely, 1924

Distribution: India, Nepal

Comments: Endemic to South Asia

6. Pardosa tridentis Caporiacco, 1935

Synonyms: Pardosa tridentia Caporiacco, 1935

Lycosa tatensis Tikader, 1964

Pardosa tatensis Tikader \& Malhotra, 1980

Distribution: India, Nepal

Comments: Endemic to South Asia. Pardosa

tatensis was synonymised with Pardosa tridentis by Buchar (1976).

IX.d. Genus Trochosa C.L. Koch, 1847

1. Trochosa gravelyi Buchar, 1976

Comments: Endemic to Nepal

X. FAMILY MYSMENIDAE PETRUNKEVITCH, 1928

X.a. Genus lardinis Simon, 1899

Comments: Endemic to South Asia.

1. Iardinis martensi Brignoli, 1978

Comments: Endemic to Nepal

\section{FAMILY NESTICIDAE Simon, 1894}

XI.a. Genus Nesticella Lehtinen \& Saaristo, 1980

1. Nesticella nepalensis (Hubert, 1973)

Synonym: Nesticus nepalensis Hubert, 1973

Comments: Endemic to Nepal. Nesticus nepalensis

was transferred to genus Nesticella by Lehtinen \& Saaristo (1980).

\section{FAMILY OCHYROCERATIDAE FAGE, 1912}

XII.a. Genus Leclercera Deeleman-Reinhold, 1995

1. Leclercera machadoi (Brignoli, 1973)

Synonym: Althepus machadoi Brignoli, 1973

Comments: Endemic to Nepal. Althepus machado was transferred to genus Leclercera by DeelemanReinhold (1995).

XII.b. Genus Psiloderces Simon, 1892

1. Psiloderces mulcatus (Brignoli, 1973)

Synonym: Althepus mulcatus Brignoli, 1973

Comments: Endemic to Nepal. Althepus mulcatus

was transferred to genus Psiloderces by DeelemanReinhold (1995).

\section{FAMILY OONOPIDAE SIMON, 1890}

XIII.a. Genus Camptoscaphiella Caporiacco, 1934

1. Camptoscaphiella silens Brignoli, 1976

Comments: Endemic to Nepal

2. Camptoscaphiella strepens Brignoli, 1976

Comments: Endemic to Nepal

XIV. FAMILY PIMOIDAE WUNDERLICH, 1986

XIV.a. Genus Pimoa Chamberlin \& Ivie, 1943

1. Pimoa nematoides Hormiga, 1994

Comments: Endemic to Nepal

\section{FAMILY PISAURIDAE SIMON, 1890}

XV.a. Genus Perenethis L. Koch, 1878

1. Perenethis sindica (Simon, 1897)

Synonym: Tetragonophthalma sindica Simon, 1897

Perenethis indica Simon, 1897

Distribution: India, Sri Lanka, Nepal, China, Philippines

XVI. FAMILY PSECHRIDAE SIMON, 1890

XVI.a. Genus Psechrus Thorell, 1878

1. Psechrus himalayanus Simon, 1906
Distribution: India, Nepal

Comments: Endemic to South Asia

2. Psechrus marsyandi Levi, 1982

Comments: Endemic to Nepa

XVII. FAMILY SALTICIDAE BLACKWALL, 1841

XVII.a. Genus Brettus Thorell, 1895

1. Brettus anchorum Wanless, 1979

Distribution: India, Nepal

Comments: Endemic to South Asia

XVII.b. Genus Carrhotus Thorell, 1891

1. Carrhotus catagraphus Jastrzebski, 1999

Comments: Endemic to Nepal

2. Carrhotus erus Jastrzebski, 1999

Comments: Endemic to Nepal

3. Carrhotus operosus Jastrzebski, 1999

Comments: Endemic to Nepal

XVII.c. Genus Chalcoscirtus Bertkau, 1880

1. Chalcoscirtus martensi Zabka, 1980

Distribution: Central Asia, Nepal, India, China

XVII.d. Genus Chinattus Logunov, 1999

1. Chinattus chichila Logunov, 2003

Comments: Endemic to Nepal

XVII.e. Genus Euophrys C. L. Koch, 1834

1. Euophrys dhaulagirica Zabka, 1980

Comments: Endemic to Nepal

2. Euophrys jirica Zabka, 1980

Comments: Endemic to Nepal

3. Euophrys nepalica Zabka, 1980

Distribution: Nepal, China

4. Euophrys omnisuperstes Wanless, 1975

Comments: Endemic to Nepal

5. Euophrys yulungensis Zabka, 1980

Distribution: China, Nepal

XVII.f. Genus Habrocestoides Prószyn'ski, 1992

Comments: This genus is Endemic to South Asia

1. Habrocestoides phulchokiensis Logunov, 1999 Comments: Endemic to Nepal

XVII.g. Genus Harmochirus Simon, 1885

1. Harmochirus zabkai Logunov, 2001

Synonyms: Harmochirus brachiatus Tikader, 1976

Harmochirus brachiatus (Tikader, 1976)

Distribution: India, Nepal, Vietnam

Comments: Logunov (2001) in describing this new species includes Harmochirus brachiatus by Tikader (1976) and Zabka (1985) as misidentifications.

XVII.h. Genus Pancorius Simon, 1902

1. Pancorius kaskiae Zabka, 1990

Comments: Endemic to Nepal

2. Pancorius minutus Zabka, 1985

Distribution: China, Nepal, Vietnam

XVII.i. Genus Phaeacius Simon, 1900

1. Phaeacius fimbriatus Simon, 1900

Distribution: Nepal, Java

2. Phaeacius saxicola Wanless, 1981

Comments: Endemic to Nepal

3. Phaeacius wanlessi Wijesinghe, 1991

Distribution: Nepal, Sri Lanka 
Comments: Endemic to South Asia

XVII.j. Genus Phintella Strand, 1906

1. Phintella suavis (Simon, 1885)

Synonym: Thiania suavis Simon, 1885

Telamonia suavis Simon, 1901

Distribution: Nepal to Malaysia

Comments: Telamonia suavis was transferred to

genus Phintella by Prószyn'ski (1984).

XVII.k. Genus Plexippoides Prószyn'ski, 1984

1. Plexippoides tristis Próchniewicz, 1990

Comments: Endemic to Nepal

XVII.I. Genus Plexippus C.L. Koch, 1846

1. Plexippus pokharae Zabka, 1990

Comments: Endemic to Nepal

XVII.m. Genus Portia Karsch, 1878

Synonym: Genus Linus Peckham \& Peckham, 1885

Comment: Genus Linus was synonymised with genus

Portia by Wanless (1978).

1. Portia fimbriata (Doleschall, 1859)

Synonym: Salticus fimbriatus Doleschall, 1859

Sinis fimbriatus (Doleschall, 1859)

Linus fimbriatus (Doleschall, 1859)

Linus alticeps Pocock, 1899

Boethoportia ocellata Hogg, 1915

Distribution: Nepal, Sri Lanka, Taiwan to Australia

XVII.n. Genus Pseudicius Simon, 1885

1. Pseudicius nepalicus (Andreeva, Heciak \&

Prószyn'ski, 1984)

Synonym: Icius nepalicus Andreeva, Heciak \&

Prószyn'ski, 1984

Distribution: India, Nepal

Comments: Endemic to South Asia

XVII.o. Genus Rhene Thorell, 1869

1. Rhene phuntsholingensis Jastrzebski, 1997

Distribution: Bhutan, Nepal

Comments: Endemic to South Asia

XVII.p. Genus Sitticus Simon, 1901

1. Sitticus niveosignatus (Simon, 1880)

Synonym: Attus niveo-signatus Simon, 1880

Attulus niveosignatus (Simon, 1880)

Distribution: Nepal to China

Comments: Attulus niveosignatus was transferred

to genus Sitticus by Prószyn'ski (1975).

XVII.r. Genus Synagelides Strand, 1906

1. Synagelides dhaulagiricus Bohdanowicz, 1987

Comments: Endemic to Nepal

2. Synagelides gorapanicus Bohdanowicz, 1987

Comments: Endemic to Nepal

3. Synagelides gosainkundicus Bohdanowicz, 1987 Comments: Endemic to Nepal

4. Synagelides himalaicus Bohdanowicz, 1987

Comments: Endemic to Nepal

5. Synagelides jiricus Bohdanowicz, 1987

Comments: Endemic to Nepal

6. Synagelides martensi Bohdanowicz, 1987

Comments: Endemic to Nepal

7. Synagelides nepalensis Bohdanowicz, 1987

Comments: Endemic to Nepal

8. Synagelides nishikawai Bohdanowicz, 1979

Comments: Endemic to Nepal
9. Synagelides oleksiaki Bohdanowicz, 1987

Comments: Endemic to Nepal

10. Synagelides thodungus Bohdanowicz, 1987

Comments: Endemic to Nepal

11. Synagelides tukchensis Bohdanowicz, 1987

Comments: Endemic to Nepal

12. Synagelides ullerensis Bohdanowicz, 1987

Comments: Endemic to Nepal

13. Synagelides walesai Bohdanowicz, 1987

Comments: Endemic to Nepal

14. Synagelides wangdicus Bohdanowicz, 1978 Comments: Endemic to Bhutan

15. Synagelides wuermlii Bohdanowicz, 1978

Comments: Endemic to Bhutan

16. Synagelides wyszynskii Bohdanowicz, 1987 Comments: Endemic to Nepal

XVII.s. Genus Yaginumaella Prószyn'ski, 1979

1. Yaginumaella nepalica Zabka, 1980

Synonym: Ptocasius nepalica Hu, 2001

Distribution: China, Nepal

2. Yaginumaella tenzingi Zabka, 1980

Comments: Endemic to Nepal

3. Yaginumaella thakkholaica Zabka, 1980 Synonym: Ptocasius thakkholaica Hu, 2001

Distribution: China, Nepal

XVIII. FAMILY SCYTODIDAE BLACKWALL, 186 XVIII.a. Genus Scytodes Latreille, 1804

1. Scytodes mawphlongensis Tikader, 1966

Synonym: Scytodes cf. strandi Brignoli, 1976

Distribution: India, Nepal

Comments: Endemic to South Asia

XIX. FAMILY SPARASSIDAE BERTKAU, 1872

Comments: Considered senior synonym of Heteropodidae by Jäger (1999).

XIX.a. Genus Bhutaniella Jäger, 2000

1. Bhutaniella hillyardi Jäger, 2000

Comments: Endemic to Nepal

2. Bhutaniella rollardae Jäger, 2001

Comments: Endemic to Nepal

XIX.b. Genus Pseudopoda Jäger, 2000

1. Pseudopoda albolineata Jäger, 2001

Comments: Endemic to Nepal

2. Pseudopoda alta Jäger, 2001

Synonym:Pseudopoda maculata Jäger, 2001

Comments: Endemic to Nepa

3. Pseudopoda ausobskyi Jäger, 2001

Comments: Endemic to Nepal

4. Pseudopoda brauni Jäger, 2001

Comments: Endemic to Nepal

5. Pseudopoda chauki Jäger, 2001

Comments: Endemic to Nepal

6. Pseudopoda chulingensis Jäger, 2001

Comments: Endemic to Nepal

7. Pseudopoda cuneata Jäger, 2001

Comments: Endemic to Nepal
8. Pseudopoda dama Jäger, 2001

Comments: Endemic to Nepal

9. Pseudopoda damana Jäger, 2001

Comments: Endemic to Nepal

10. Pseudopoda dhulensis Jäger, 2001

Synonym: Pseudopoda dhorpatan Jäger, 2001

Comments: Endemic to Nepal

11. Pseudopoda diversipunctata Jäger, 2001

Comments: Endemic to Nepal

12. Pseudopoda everesta Jäger, 2001

Comments: Endemic to Nepal

13. Pseudopoda grasshoffi Jäger, 2001

Comments: Endemic to Nepal

14. Pseudopoda heteropodoides Jäger, 2001

Comments: Endemic to Nepal

15. Pseudopoda huberti Jäger, 2001

Comments: Endemic to Nepal

16. Pseudopoda hyatti Jäger, 2001

Synonym: Pseudopoda jalja Jäger, 2001

Comments: Endemic to Nepal

17. Pseudopoda jirensis Jäger, 2001

Comments: Endemic to Nepal

18. Pseudopoda kalinchoka Jäger, 2001

Synonym: Pseudopoda nepalensis Jäger, 2001

Pseudopoda bhotensis Jäger, 2001

Comments: Endemic to Nepal

19. Pseudopoda khimtensis Jäger, 2001

Comments: Endemic to Nepa

20. Pseudopoda latembola Jäger, 2001

Comments: Endemic to Nepa

21. Pseudopoda marmorea Jäger, 2001

Comments: Endemic to Nepal

22. Pseudopoda martensi Jäger, 2001

Comments: Endemic to Nepal

23. Pseudopoda martinae Jäger, 2001

Comments: Endemic to Nepal

24. Pseudopoda monticola Jäger, 2001

Comments: Endemic to Nepal

25. Pseudopoda schawalleri Jäger, 2001

Comments: Endemic to Nepal

26. Pseudopoda sinopodoides Jäger, 2001

Comments: Endemic to Nepal

27. Pseudopoda tinjura Jäger, 2001

Comments: Endemic to Nepal

28. Pseudopoda triapicata Jäger, 2001

Comments: Endemic to Nepal

29. Pseudopoda trisuliensis Jäger, 2001

Comments: Endemic to Nepal

30. Pseudopoda varia Jäger, 2001

Synonym: Pseudopoda montana Jäger, 2001

Pseudopoda dhorpara Jäger, 2001

Comments: Endemic to Nepal. According to Platnick

(2006), Pseudopoda montana and Pseudopoda

dhorpara were invalid provisional names for

Pseudopoda varia. 
XX. FAMILY TETRABLEMMIDAE O.P.- CAMBRIDGE, 3. Xysticus dolpoensis Ono, 1978 1873

XX.a. Genus Brignoliella Shear, 1978

1. Brignoliella martensi (Brignoli, 1972)

Synonym: Paculla martensi Brignoli, 1972

Comments: Endemic to Nepal. Paculla martensi was

transferred to the genus Brignoliella by Shear (1978).

XX.b. Genus Tetrablemma O.P.-Cambridge, 1873

1. Tetrablemma phulchoki Lehtinen, 1981

Comments: Endemic to Nepal

XXI. FAMILY THERAPHOSIDAE THORELL, 1870

XXI.a. Genus Haplocosmia Schmidt \& von Wirth, 1996

Comment: Endemic to South Asia

1. Haplocosmia nepalensis Schmidt \& von Wirth, 1996 Comments: Endemic to Nepal

XXII. FAMILY THOMISIDAE SUNDEVALL, 1833

XXII.a. Genus Lysiteles Simon, 1895

1. Lysiteles annapurnus Ono, 1979

Comments: Endemic to Nepal

2. Lysiteles himalayensis Ono, 1979

Distribution: Nepal, Bhutan

Comments: Endemic to South Asia

3. Lysiteles lepusculus Ono, 1979

Comments: Endemic to Nepal

4. Lysiteles maior Ono, 1979

Synonym: Lysiteles maius Ono, 1979

Distribution: Russia, Nepal to Japan

5. Lysiteles montivagus Ono, 1979

Comments: Endemic to Nepal

6. Lysiteles niger Ono, 1979

Distribution: Bhutan, Nepal

Comments: Endemic to South Asia

7. Lysiteles parvulus Ono, 1979

Comments: Endemic to Nepal

8. Lysiteles saltus Ono, 1979

Synonym: Xysticus himalayaensis $\mathrm{Hu} \& \mathrm{Li}, 1987$

Xysticus mandali Hu \& Li, 1987

Distribution: Nepal, Bhutan, China

XXII.b. Genus Monaeses Thorell, 1869

Synonym: Genus Mecostrabus Simon, 1903

Comments: The genus Mecostrabus was synonymised with the genus Monaeses by Ono (1985).

1. Monaeses aciculus (Simon, 1903)

Synonym: Mecostrabus aciculus Simon, 1903

Monaeses acciculus Tang \& Song, 1988

Distribution: Nepal to Japan, Philippines

XXII.c. Genus Stiphropus Gerstäcker, 1873

1. Stiphropus soureni Sen, 1964

Distribution: India, Nepal, Bhutan

Comments: Endemic to South Asia

XXII.d. Genus Xysticus C.L. Koch, 1835

1. Xysticus alpinistus Ono, 1978

Distribution: Nepal, China

2. Xysticus croceus Fox, 1937

Synonyms: Xysticus ephippiatus Bösenberg \&

Strand, 1906

Xysticus sujatai Tikader, 1962

Distribution: India, Nepal, Bhutan, China, Korea, Japan

Comments: Xysticus sujatai was synonymised

with Xysticus croceus by Ono (1988).
Distribution: Nepal, China

4. Xysticus elephantus Ono, 1978

Distribution: Nepal, China

5. Xysticus martensi Ono, 1978

Comments: Endemic to Nepal

6. Xysticus nepalhimalaicus Ono, 1978

Comments: Endemic to Nepal

7. Xysticus potamon Ono, 1978

Comments: Endemic to Nepal

8. Xysticus roonwali Tikader, 1964

Distribution: India, Nepal

Comments: Endemic to South Asia

9. Xysticus simplicipalpatus Ono, 1978

Distribution: Nepal, Bhutan

Comments: Endemic to South Asia

XXIII. FAMILY ZODARIIDAE THORELL, 1881

XXIII.a. Genus Storena Walckenaer, 1805

Comments: According to Platnick (2006), Indian species under this genus are probably misplaced. Further taxonomic verification required.

1. Storena erratica Ono, 1983

Comments: Endemic to Nepal

2. Storena martensi Ono, 1983

Comments: Endemic to Nepal

3. Storena nepalensis Ono, 1983

Comments: Endemic to Nepal

4. Storena uncinata Ono, 1983

Comments: Endemic to Nepal

XXIII.b. Genus Suffasia Jocqué, 1991

Comments: Endemic to South Asia.

1. Suffasia martensi Ono, 2006

Comments: Endemic to Nepal

2. Storena kanchenjunga Ono, 2006

Comments: Endemic to Nepal

3. Suffasia tumegaster Jocqué, 1992

Comments: Endemic to Nepal

$$
\text { Nepal spider summary }
$$

Number of Families: 23

Number of Genera: 79

Number of Species: 222

Number of Subspecies: 0

Number of Endemic Families: 0

Number of Endemic Genera: 4

Number of Endemic Species: 176

\section{Checklist 7. Spiders of Pakistan}

I. FAMILY ARANEIDAE SIMON, 1895

I.a. Genus Araneus Clerck, 1757

1. Araneus alboquadratus Dyal, 1935

Comments: Endemic to Pakistan

2. Araneus camilla (Simon, 1889)

Synonym: Epeira camilla Simon, 1889

Distribution: India, Pakistan

Comments: Endemic to South Asia

3. Araneus formosellus (Roewer, 1942)

Synonym: Araneus formosus Dyal, 1935

Aranea formosella Roewer, 1942

Comments: Endemic to Pakistan. Araneus formosus

Dyal, 1935 was preoccupied by Olivier, 1789 and thus, Roewer (1942) provided a replacement name Aranea formosella for this species. Platnick (2006) corrected the species name as per ICZN rules.

4. Araneus fulvellus (Roewer, 1942)

Synonyms: Araneus fulvus Dyal, 1935

Aranea fulvella Roewer, 1942

Distribution: India, Pakistan

Comments: Endemic to South Asia. As species name was preoccupied by Walckenaer (1842) under genus Epeira, Roewer (1942) provided a replacement name Araneus fulvell for Araneus fulvus.

5. Araneus nympha (Simon, 1889)

Synonym: Epeira nympha Simon, 1889

Distribution: India, Pakistan, China

Comments: Epeira nympha was transferred to

genus Araneus by Tikader (1977) without any explanation for the transfer.

I.b. Genus Argiope Audouin, 1826

1. Argiope anasuja Thorell, 1887

Synonyms: Argiope anasuja fletcheri Hirst, 1911

Argiope plagiata Karsch, 1891

Distribution: India, Maldives, Pakistan

Comments: Endemic to South Asia

I.c. Genus Cyclosa Menge, 1866

1. Cyclosa chichawatniensis Mukhtar \& Mushtaq, 2005

Comments: Endemic to Pakistan

2. Cyclosa hexatuberculata Tikader, 1982

Distribution: India, Pakistan

Comments: Endemic to South Asia. According to

Platnick (2006), this species occurs in Pakistan.

3. Cyclosa krusa Barrion \& Litsinger, 1995

Distribution: Pakistan, Philippines

Comments: According to Platnick (2006), this species occurs in Pakistan.

4. Cyclosa mohini Dyal, 1935

Comments: Endemic to Pakistan

5. Cyclosa punjabiensis Ghafoor \& Beg, 2002

Comments: Endemic to Pakistan

6. Cyclosa saismarka Barrion \& Litsinger, 1995

Distribution: Pakistan, Philippines

Comments: According to Platnick (2006), this species occurs in Pakistan.

7. Cyclosa spirifera Simon, 1889

Distribution: India, Pakistan

Comments: Endemic to South Asia. According to

Platnick (2006), this species occurs in Pakistan.

I.d. Genus Cyrtophora Simon, 1864

1. Cyrtophora cicatrosa (Stoliczka, 1869)

Synonyms: Epeira cicatrosa Stoliczka, 1869

Epeira salebrosa Thorell, 1878 
Euetria salebrosa (Thorell, 1878)

Meta adspersata Karsch, 1891

Araneus cicatrosa (Stoliczka, 1869)

Cyrtophora salebrosa (Thorell, 1878)

Cyrtophora cicatorosa (Stoliczka, 1869)

Distribution: Pakistan to New Guinea

I.e. Genus Eriovixia Archer, 1951

1. Eriovixia excelsa (Simon, 1889)

Synonyms: Glyptogona excelsa Simon 1889

Araneus excelsus (Simon 1889)

Neoscona excelsus (Simon 1889)

Distribution: India, Pakistan, Philippines, Indonesia,

Taiwan

Comments: Neoscona excelsus was transferred to genus Eriovixia by Grasshoff (1986).

I.f. Genus Gasteracantha Sundevall, 1833

1. Gasteracantha dalyi Pocock, 1900

Distribution: India, Pakistan

Comments: Endemic to South Asia

I.g. Genus Neoscona Simon, 1864 1. Neoscona pavida (Simon, 1906)

Synonyms: Araneus pavidus Simon, 1906

Distribution: India, Pakistan, China

Comment: Araneus pavidus was transferred to genus Neoscona by Tikader \& Bal, 1981.

\section{FAMILY CLUBIONIDAE WAGNER, 1887}

II.a. Genus Clubiona Latreille, 1804

1. Clubiona filicata O. P.-Cambridge, 1874

Synonym: Clubiona swatowensis Strand, 1907

Distribution: India, Bangladesh, Pakistan, China

Comments: According to Platnick (2006), this species

occurs in Pakistan.

2. Clubiona kasurensis Mukhtar \& Mushtaq, 2005

Comments: Endemic to Pakistan

\section{FAMILY CORINNIDAE KARSCH, 1880}

Comments: Considered valid by Wunderlich (1986), Synonym: Zelotus faisalabadensis Butt \& Beg, 2004 so genera listed by Brignoli under Castianeirinae and Comments: Endemic to Pakistan. Generic name Corinninae (Clubionidae) are treated here and also genera listed by Roewer under the Corinninae have been apportioned to the Corinnidae (Platnick, 2006).

III.a. Genus Corinna C.L. Koch, 1841

1. Corinna propera (Dyal, 1935)

Synonym: Lausus properus Dyal, 1935

Comments: Endemic to Pakistan. According to Bonaldo (2000), this species is misplaced in this genus and probably belong to Oedignatha group (Platnick, 2006).

IV. FAMILY DICTYNIDAE O.P.-CAMBRIDGE, 1871

IV.a. Genus Dictyna Sundevall, 1833

1. Dictyna albida O. P.-Cambridge, 1885

Distribution: India, China, Pakistan

\section{FAMILY ERESIDAE C.L. KOCH, 1851}

V.a. Genus Stegodyphus Simon, 1873

1. Stegodyphus pacificus Pocock, 1900

Distribution: India, Iran, Pakistan

\section{FAMILY GNAPHOSIDAE POCOCK, 1898}

VI.a. Genus Drassodes Westring, 1851

1. Drassodes lutescens (C. L. Koch, 1839)

Synonyms: Drassus lutescens C.L. Koch, 1839

Drassodes maindroni Simon, 1897

Drassodes speculator Kulczyn'ski, 1899

Drassodes persimilis Denis, 1937

Drassodes mazurae Esyunin \& Tuneva, 2002
Distribution: Mediterranean to Pakistan with Drassus lutescens Levy (2004).

2. Drassodes parvidens Caporiacco, 1934

Distribution: India, Pakistan

Comments: Endemic to South Asia

3. Drassodes rubicundulus Caporiacco, 1934

Distribution: India, Pakistan

Comments: Endemic to South Asia

VI.b. Genus Gnaphosa Latreille, 1804

1. Gnaphosa eucalyptus Ghafoor \& Beg, 2002

Comments: Endemic to Pakistan

2. Gnaphosa pumila Dyal, 1935

Comments: Endemic to Pakistan

VI.c. Genus Scotophaeus Simon, 1893

1. Scotophaeus faisalabadiensis Ghafoor \& Beg, 2002

Comments: Endemic to Pakistan

VI.d. Genus Setaphis Simon, 1893

1. Setaphis browni (Tucker, 1923)

Synonyms: Camillina browni Tucker, 1923

Liodrassus mandae Tikader \& Gajbe, 1977

Nodocion mandae (Tikader \& Gajbe, 1977)

Distribution: India, Central, South Africa to Pakistan

Comments: Liodrassus mandae was synonymised with Setaphis browni by Platnick \& Murphy (1996).

VI.e. Genus Synaphosus Platnick \& Shadab, 1980 1. Synaphosus neali Ovtsharenko, Levy \& Platnick, 1994 Distribution: Iran, Pakistan

VI.f Genus Talanites Simon, 1893

1. Talanites tibialis Caporiacco, 1934

Distribution: India, Pakistan

Comments: Endemic to South Asia

VI.g. Genus Zelotes Gistel, 1848

Comments: Endemic to Pakistan. Generic name

2. Zelotes illustris Butt \& Beg, 2004

Synonym: Zelotus illustris Butt \& Beg, 2004

Comments: Endemic to Pakistan. Generic name mispelled in original text.

3. Zelotes pakistaniensis Butt \& Beg, 2004

Synonym: Zelotus pakistaniensis Butt \& Beg, 2004

Comments: Endemic to Pakistan. Generic name mispelled in original text.

4. Zelotes pulchellus Butt \& Beg, 2004

Synonym: Zelotus pulchellus Butt \& Beg, 2004

Comments: Endemic to Pakistan. Generic name mispelled in original text.

5. Zelotes sarawakensis (Thorell, 1890)

Synonym: Prosthesima sarawakensis Thorell, 1890 Prosthesima iusta Kulczyn'ski, 1911

Distribution: Pakistan to Borneo and Australia

Comments: Prosthesima iusta was synonymised with Zelotes sarawakensis by Platnick \& Ovtsharenko (1995).

6. Zelotes sindi Caporiacco, 1934

Distribution: India, Pakistan

Comments: Endemic to South Asia. Pakistan in

distribution was missed out in Siliwal et al. (2005).

VII. FAMILY IDIOPIDAE SIMON, 1892

VII.a. Genus Idiops Perty, 1833
Comments: Raven (1985) transferred genus Idiops framily Ctenizidae to family Idiopidae. According to Platnick (2006), many species of the genus Acanthodon (Ctenizidae) were transferred to genus Idiops.

1. Idiops designatus O.P.-Cambridge, 1885 Synonym: Acanthodon designatus Pocock, 1900 Comments: Endemic to Pakistan

\section{FAMILY LYCOSIDAE SUNDEVALL, 1833}

VIII.a. Genus Anomalosa Roewer, 1960

1. Anomalosa harishi (Dyal, 1935)

Synonym: Anomalomma harishi Dyal, 1935

Comments: Endemic to Pakistan. Roewer (1960)

transferred Anomalomma harishi to genus Anomalosa.

VIII.b. Genus Arctosa C.L. Koch, 1847

1. Arctosa mulani (Dyal, 1935)

Synonym: Pardosa mulani Dyal, 1935

Distribution: India, Pakistan

Comments: Endemic to South Asia. Pardosa mulan was transferred to genus Arctosa by Tikader and Malhotra (1980).

VIII.c. Genus Evippa Simon, 1882 Synonym: Evippella Strand, 1906

Comments: Evippella was synonymised with genus Evippa by Alderweireldt (1991)

1. Evippa praelongipes (O.P.-Cambridge, 1870) Synonyms: Lycosa praelongipes O.P.-Cambridge, 1870 Pardosa praelongipes Schmidt, 1895 Distribution: Egypt to India, Pakistan, Kazakhstan

VIII.d. Genus Hippasa Simon, 1885 1. Hippasa pisaurina Pocock, 1900 Distribution: Iraq, India, Pakistan

VIII.e. Genus Lycosa Latreille, 1804

Synonym: Genus Allohogna Roewer, 1955

Comments: Allohogna was synonymised with genus Lycosa by Fuhn \& Niculescu-Burlacu (1971).

1. Lycosa chaperi Simon, 1885

Synonym: Hogna chaperi (Simon, 1885)

Distribution: India, Pakistan

Comments: Endemic to South Asia. Pakistan was not included in distribution by Siliwal et al. (2005).

2. Lycosa kempi Gravely, 1924

Synonym: Piratula kempi (Gravely, 1924)

Distribution: India, Pakistan, Bhutan, China

Comments: Transfer of Lycosa kempi to genus Piratula was considered incorrect and thus the original name was reinstated by Tikader (1970).

3. Lycosa mackenziei Gravely, 1924

Synonym: Pardosa mackenziei (Gravely, 1924)

Distribution: Pakistan, India, Bangladesh

Comments: Endemic to South Asia. Transfer of Lycosa mackenziei to genus Pardosa was considered incorrect and thus, original name was reinstated by Tikader and Malhotra (1980).

VIII.f. Genus Ocyale Audouin, 1826

1. Ocyale kumari Dyal, 1935

Comments: Endemic to Pakistan

VIII.g. Genus Pardosa C. L. Koch, 1847

1. Pardosa basiri (Dyal, 1935)

Synonym: Lycosa basiri Dyal, 1935

Comments: Endemic to Pakistan. Lycosa basiri was transferred to genus Pardosa by Roewer (1955).

2. Pardosa birmanica Simon, 1884

Synonyms: Lycosa ipnochoera Thorell, 1890 
Lycosa birmanica Simon, 1884 Lycosa subbirmanica Strand, 1909

Pardosa bhatnagari Sadana, 1971

Distrbution: Pakistan to China, Philippines, Sumatra

Comments: Transfer of Pardosa birmanica to genus Lycosa was considered incorrect and thus, original name was reinstated by Buchar (1976)

3. Pardosa fletcheri (Gravely, 1924)

Synonyms: Lycosa fletcheri Gravely, 1924

Lycosa rothaka Tikader, 1970

Distribution: India, Nepal, Pakistan

Comments: Endemic to South Asia. Lycosa rothaka

was synonymised with Pardosa fletcheri by Tikader \& Malhotra (1980).

4. Pardosa lahorensis Dyal, 1935

Distribution: India, Pakistan

Comments: Endemic to South Asia

5. Pardosa leucopalpis Gravely, 1924

Distribution: India, Pakistan, Sri Lanka

Comments: Endemic to South Asia

6. Pardosa oakleyi Gravely, 1924

Distribution: Pakistan, India, Bangladesh

Comments: Endemic to South Asia

7. Pardosa pseudoannulata (Bösenberg \& Strand, 1906) Synonyms: Tarentula pseudoannulata Bösenberg \& Strand, 1906

Lycosa doenitzi Bösenberg \& Strand, 1906

Lycosa innominabilis Dönitz \& Strand, in Bösenberg \&

Strand, 1906

Lycosa subtarentula Dönitz \& Strand, in Bösenberg \& Strand, 1906

Lycosa pseudoterricola Schenkel, 1936

Pardosa doenitzi (Bösenberg \& Strand, 1906)

Pardosa innominabilis (Dönitz \& Strand, in Bösenberg

\& Strand, 1906)

Pardosa subtarentula (Dönitz \& Strand, in Bösenberg \& Strand, 1906)

Avicosa pseudoannulata (Bösenberg \& Strand, 1906)

Avicosa pseudoterricola (Schenkel, 1936)

Lycosa cinnameovittata Schenkel, 1963

Pardosa annandalei Tikader \& Malhotra, 1980

Distribution: Pakistan to Japan, Philippines, Java

Comments: Pardosa annandalei was synonymised

with Pardosa pseudoannulata by Yu \& Song (1988).

8. Pardosa timidula (Roewer, 1951)

Synonyms: Lycosa timida Simon, 1882

Pardosa timida (Simon, 1882)

Lycosa timidula Roewer, 1951

Hogna timidula (Roewer, 1951)

Allocosa timidula (Roewer, 1951)

Pardosa timida (Simon, 1882)

Distribution: Yemen, Sri Lanka, Pakistan

Comments: The species name Lycosa timida was preoccupied by Lucas (1846) and thus Roewer (1951) provided replacement name Lycosa timidula for Lycosa timida. Allocosa timida was transferred to genus Pardosa by Tikader and Malhotra (1980).

\section{FAMILY MIMETIDAE SIMON, 1881}

IX.a. Genus Gelanor Thorell, 1869

1. Gelanor muliebris Dyal, 1935

Comments: Endemic to Pakistan

\section{FAMILY MITURGIDAE SIMON, 1885}

X.a. Genus Strotarchus Simon, 1888

1. Strotarchus alboater Dyal, 1935

Comments: Endemic to Pakistan

2. Strotarchus vittatus Dyal, 1935

Comments: Endemic to Pakistan

\section{FAMILY NEPHILIDAE SIMON, 1894}

Comments: Subfamily Nephilinae under family Tetragnathidae was elevated to family level by Kuntner (2006). Genera Clitaetra, Herennia, Nephila and Nephilengys were transferred from family Tetragnathidae to this family.

XI.a. Genus Nephila Leach, 1815

1. Nephila clavata L. Koch, 1878

Synonyms: Nephila limbata Thorell, 1898

Nephila obnubila Simon, 1906

Distribution: Bhutan, Pakistan, India to Japan

2. Nephila pakistaniensis Ghafoor \& Beg, 2002

Comments: Endemic to Pakistan

XII. FAMILY OECOBIIDAE BLACKWALL, 1862

XII.a. Genus Uroctea Dufour, 1820

Comments: Tikader (1987) considered this genus in

the family Urocteidae, which was a wrong placement.

1. Uroctea matthaii Dyal, 1935

Comments: Endemic to Pakistan

XIII. FAMILY OXYOPIDAE THORELL, 1870

XIII.a. Genus Oxyopes Latreille, 1804

1. Oxyopes campii Mushtaq \& Qadar, 1999

Comments: Endemic to Pakistan

2. Oxyopes gossypae Mushtaq \& Qadar, 1999

Comments: Endemic to Pakistan

3. Oxyopes hindostanicus Pocock, 1901 Distribution: India, Sri Lanka, Pakistan

Comments: Endemic to South Asia. Pakistan was not included in distribution by Siliwal et al. (2005).

4. Oxyopes jubilans O.P.-Cambridge, 1885

Distribution: India, Pakistan, China

5. Oxyopes oryzae Mushtaq \& Qadar, 1999

Comments: Endemic to Pakistan

6. Oxyopes raviensis Dyal, 1935

Comments: Endemic to Pakistan

7. Oxyopes ryvesi Pocock, 1901

Synonyms Oxyopes ryvesii Pocock, 1901

Distribution: India, Pakistan

Comments: Endemic to South Asia. Pakistan was not included in distribution by Siliwal et al. (2005).

8. Oxyopes wroughtoni Pocock, 1901

Distribution: India, Pakistan

Comments: Endemic to South Asia. Pakistan was not included in distribution by Siliwal et al. (2005).

XIV. FAMILY PHILODROMIDAE THORELL, 1870 XIV.a. Genus Thanatus C.L. Koch, 1837

Comments: Removed from the Thomisidae and placed under Philodromidae by Homann (1975).

1. Thanatus fornicatus Simon, 1897

Distribution: Israel, Pakistan

XV. FAMILY PISAURIDAE SIMON, 1890

XV.a. Genus Perenethis L. Koch, 1878

1. Perenethis dentifasciata (O. P.-Cambridge, 1885)

Synonym: Ocyale dentifasciata O. P.-Cambridge, 1885

Pisaura dentifasciata (O. P.-Cambridge, 1885)

Distribution: India or Pakistan

Comments: Endemic to South Asia. Pisaura

dentifasciata was transferred to genus Perenethis

by Sierwald (1989).
XVI. FAMILY PRODIDOMIDAE SIMON, 1884

XVI.a. Genus Prodidomus Hentz, 1847

1. Prodidomus margala Platnick, 1976

Comments: Endemic to Pakistan

XVII. FAMILY SALTICIDAE BLACKWALL, 1841

XVII.a. Genus Aelurillus Simon, 1884

1. Aelurillus logunovi Azarkina, 2004

Distribution: Afghanistan, Pakistan

Comments: Endemic to South Asia

XVII.b. Genus Akela Peckham \& Peckham, 1896

1. Akela fulva Dyal, 1935

Comments: Endemic to Pakistan

XVII.c. Genus Bellota Peckham \& Peckham, 1892

1. Bellota fascialis Dyal, 1935

Comments: Endemic to Pakistan

2. Bellota livida Dyal, 1935

Comments: Endemic to Pakistan

XVII.d. Genus Chrysilla Thorell, 1887

1. Chrysilla albens Dyal, 1935

Comments: Endemic to Pakistan

XVII.e. Genus Cosmophasis Simon, 1901

1. Cosmophasis umbratica Simon, 1903

Distribution: Pakistan to Sumatra

XVII.f. Genus Cotinusa Simon, 1900

1. Cotinusa splendida (Dyal, 1935)

Synonym: Gophoa splendida Dyal, 1935

Comments: Endemic to Pakistan. According to

Platnick (2006), Gophoa splendida was transferred

to genus Cotinusa.

XVII.g. Genus Euophrys C. L. Koch, 1834

1. Euophrys auricolor Dyal, 1935

Comments: Endemic to Pakistan

2. Euophrys rubroclypea Dyal, 1935

Comments: Endemic to Pakistan

3. Euophrys rufa Dyal, 1935

Comments: Endemic to Pakistan

XVII.h. Genus Flacillula Strand, 1932

1. Flacillula purpurea (Dyal, 1935)

Synonym: Flacilla purpurea Simon, 1901

Comments: Endemic to Pakistan

XVII.i. Genus Freya C. L. Koch, 1850

1. Freya dyali Roewer, 1951

Synonym: Freya trifasciata Dyal, 1935

Comments: Endemic to Pakistan. Freya trifasciata was preoccupied by C. L. Koch, 1846 and thus Roewer (1951) provided replacement name Freya dyali for Freya trifasciata.

XVII.j. Genus Habrocestum Simon, 1876

1. Habrocestum dyali Roewer, 1955

Synonym: Habrocestum rubroclypeatum Dyal, 1935 Comments: Endemic to Pakistan. Habrocestum rubroclypeatum was preoccupied by Lessert (1927) and thus Roewer (1955) provided replacement name Habrocestum dyali for Habrocestum rubroclypeatum.

2. Habrocestum panjabium Roewer, 1951

Synonym: Habrocestum algericum Dyal, 1935

Habrocestum panjabius Roewer, 1951

Comments: Endemic to Pakistan. Habrocestum algericum was preoccupied by Dalmas (1920) and thus Roewer (1951) provided replacement name Habrocestum panjabius for Habrocestum algericum. 
XVII.k. Genus Holcolaetis Simon, 1885 1. Holcolaetis dyali Roewer, 1951

Synonym: Holcolaetis vidua Dyal, 1935

Comments: Endemic to Pakistan. Holcolaetis vidua was preoccupied by Lessert (1927) and thus Roewer (1951) provided replacement name Holcolaetis dyali for Holcolaetis vidua.

XVII.I. Genus Jollas Simon, 1901

Synonym: Genus Oningis Simon, 1901

Comment: The genus Oningis was synonymised with the genus Jollas by Galiano (1991).

1. Jollas lahorensis (Dyal, 1935)

Synonym: Oningis lahorensis Dyal, 1935

Comments: Endemic to Pakistan

XVII.m. Genus Marpissa C.L. Koch, 1846

1. Marpissa carinata Butt \& Beg, 2000

Comments: Endemic to Pakistan

2. Marpissa fornicis (Dyal, 1935)

Synonym: Hyctia fornicis Dyal, 1935

Comments: Endemic to Pakistan. Hyctia fornicis was transferred to genus Marpissa by Harm (1981).

3. Marpissa insignis Butt \& Beg, 2000

Comments: Endemic to Pakistan

4. Marpissa mirabilis Butt \& Beg, 2000

Comments: Endemic to Pakistan

5. Marpissa tenebrosa Butt \& Beg, 2000

Comments: Endemic to Pakistan

XVII.n. Genus Menemerus Simon, 1868

1. Menemerus raji Dyal, 1935

Comments: Endemic to Pakistan

XVII.o. Genus Myrmarachne MacLeay, 1839

1. Myrmarachne laeta (Thorell, 1887)

Synonyms: Ascalus laeta Thorell, 1887

Synemosyna laeta (Thorell, 1887)

Distribution: India, Pakistan, Nias Island, China

2. Myrmarachne orientales Tikader, 1973

Synonym: Myrmarachne orientalis Brignoli, 1983

Distribution: Pakistan, India

Comments: Endemic to South Asia

3. Myrmarachne ramunni Narayan, 1915

Distribution: India, Pakistan

Comments: Endemic to South Asia. Pakistan was not included in distribution bySiliwal et al. (2005).

XVII.p. Genus Pellenes Simon, 1876

1. Pellenes dyali Roewer, 1951

Synonym: Habrocestum coronatum Dyal, 1935

Comments: Endemic to Pakistan. Habrocestum coronatum was preoccupied by Peckham \& Peckham (1888) and thus Roewer (1951) provided replacement name Pellenes dyali for Habrocestum coronatum.

XVII.q. Genus Penionomus Simon, 1903

1. Penionomus dyali Roewer, 1951

Synonym: Penionomus longipalpis Dyal, 1935

Comments: Endemic to Pakistan. Penionomus longipalpis was preoccupied by Simon (1889) and thus Roewer (1951) provided replacement name Penionomus dyali for Penionomus longipalpis.

XVII.r. Genus Perenethis L. Koch, 1878

1. Perenethis dentifasciata (O. P.-Cambridge, 1885)

Synonym: Ocyale dentifasciata O. P.-Cambridge, 1885

Pisaura dentifasciata (O. P.-Cambridge, 1885)

Distribution: India or Pakistan

Comments: Endemic to South Asia. Pisaura

dentifasciata was transferred to genus Perenethis by Sierwald (1989).

XVII.s. Genus Phlegra Simon, 1876

1. Phlegra dhakuriensis (Tikader, 1974)

Synonym: Marpissa dhakuriensis Tikader, 1974

Distribution: India, Pakistan

Comments: Endemic to South Asia. Marpissa dhakuriensis was transferred to genus Phlegra by Nenilin (1984a).

2. Phlegra swanii Mushtaq, Beg \& Waris, 1995 Comments: Endemic to Pakistan

XVII.t. Genus Pilia Simon, 1902

1. Pilia escheri Reimoser, 1934

Comments: Endemic to Pakistan

XVII.u. Genus Pseudicius Simon, 1885

1. Pseudicius flavipes (Caporiacco, 1935)

Synonyms: Icius flavipes Caporiacco, 1935

Distribution: Turkmenistan, Pakistan

Comments: Icius flavipes was transferred to genus

Pseudicius by Prószyn'ski (1990).

2. Pseudicius frigidus (O.P.-Cambridge, 1885)

Synonyms: Menemerus frigidus O.P.-Cambridge, 1885

Phlegra icioides Simon, 1889

Icius icioides (Simon, 1889)

Icius frigidus (O.P.-Cambridge, 1885)

Distribution: Afghanistan, Pakistan, India, China

Comments: Icius icioides was synonymised with

Pseudicius frigidus by Andreeva et al. (1984).

XVII.v. Genus Pystira Simon, 1901

1. Pystira versicolor Dyal, 1935

Comments: Endemic to Pakistan

XVII.w. Genus Sitticus Simon, 1901

1. Sitticus dyali Roewer, 1951

Synonym: Sitticus niger Dyal, 1935

Comments: Endemic to Pakistan. preoccupied by Reimoser (1919) and thus, Roewe (1951) provided a replacement name Sitticus dyali for Sitticus niger.

XVII.x. Genus Thiania C.L. Koch, 1846

1. Thiania aura Dyal, 1935

Comments: Endemic to Pakistan

XVIII. FAMILY SCYTODIDAE BLACKWALL, 1864

XVIII.a. Genus Scytodes Latreille, 1804

1. Scytodes propinqua Stoliczka, 1869

Distribution: India, Pakistan

Comments: Endemic to South Asia. Pakistan was not included in distribution by Siliwal et al. (2005).

2. Scytodes sordida Dyal, 1935

Comments: Endemic to Pakistan

XIX. FAMILY SPARASSIDAE BERTKAU, 1872

Comments: Considered senior synonym Heteropodidae by Jäger (1999)

XIX.a. Genus Heteropoda Latreille, 1804

Synonyms: Genus Torania Simon, 1886

Genus Panaretus Simon, 1880

Comments: Genus Torania was synonymised with genus Heteropoda by Jäger (2001) and genus Panaretus was synonymised with genus Heteropoda by Jäger (2002).

1. Heteropoda afghana Roewer, 1962 Distribution: Afghanistan, Pakistan, India Comments: Endemic to South Asia
XIX.b. Genus Olios Walckenaer, 1837

1. Olios fugax (O.P.-Cambridge, 1885)

Synonym: Sparassus fugax O.P.-Cambridge, 1885

Distribution: Pakistan, Yarkand

2. Olios iranii (Pocock, 1901)

Synonym: Sparassus iranii Pocock, 1901

Distribution: India, Pakistan

Comments: Endemic to South Asia. Sparassus iranii was transferred to genus Olios by Gravely (1931).

3. Olios lutescens (Thorell, 1894)

Synonym: Midamus lutescens Thorell, 1894

Sparassus lutescens (Thorell, 1894)

Distribution: Pakistan, Myanmar, Sumatra, Java

Comments: Sparassus lutescens was transferred to

genus Olios by Dyal (1935).

4. Olios punjabensis Dyal, 1935

Comments: Endemic to Pakistan

\section{Olios tener (Thorell, 1891)}

Synonym: Sparassus tener Thorell, 1891

Distribution: Pakistan, India, Myanmar

Comments: Sparassus tener was transferred to genus Olios by Gravely (1931).

XIX.c. Genus Pseudopoda Jäger, 2000

1. Pseudopoda prompta (O.P.-Cambridge, 1885)

Synonyms: Sarotes promptus O.P.-Cambridge, 1885

Heteropoda smythiesi Simon, 1897

Heteropoda prompta (O.P.-Cambridge, 1885)

Distribution: India, Pakistan

Comments: Endemic to South Asia. Heteropoda smythiesi (Heterpodidae) was synonymised with Pseudopoda prompta and Heteropoda prompta (Heteropodidae) was transferred to genus Pseudopoda by Jäger (2000).

XIX.d. Genus Sivalicus Dyal, 1957

Comments: This genus is monotypic and endemic to Pakistan.

1. Sivalicus viridis Dyal, 1957

Comments: Endemic to Pakistan

XIX.e. Genus Spariolenus Simon, 1880

1. Spariolenus tigris Simon, 1880

Synonym: Spariolenus petricola Gravely, 1931

Distribution: India, Pakistan, Malaysia

Comments: Spariolenus petricola was synonymised with Spariolenus tigris by Jäger (2002).

XIX.f. Genus Thelcticopis Karsch, 1884

1. Thelcticopis ancorum Dyal, 1935

Comments: Endemic to Pakistan

2. Thelcticopis telonotata Dyal, 1935

Comments: Endemic to Pakistan

XX. FAMILY THERAPHOSIDAE THORELL, 1870

XX.a. Genus Selenocosmia Ausserer, 1871

1. Selenocosmia pritami Dyal, 1935

Comments: Endemic to Pakistan

XXI. FAMILY THERIDIIDAE SUNDEVALL, 1833

XXI.a. Genus Achaearanea Strand, 1929

1. Achaearanea tesselata (Keyserling, 1884)

Synonym: Theridion tesselatum Keyserling, 1884

Theridion picadoi Banks, 1909

Achaearanea terex Levi, 1959

Achaearanea picadoi (Banks, 1909)

Achaearanea mundula Chrysanthus, 1963

Distribution: Mexico to Paraguay, New Guinea, Pakistan 
XXI.b. Genus Argyrodes Simon, 1864 Synonyms: Genus Argyrodina Strand, 1926 Comments: Genera Argyrodina was synonymised with genus Argyrodes by Levi \& Levi (1962).

1. Argyrodes flavescens O.P.-Cambridge, 1880 Synonyms: Argyrodes sumatranus Thorell, 18 Argyrodes miniaceus Bösenberg \& Strand, 1906 Distribution: India, Pakistan, Sri Lanka to Korea, Japan, New Guinea

XXI.c. Genus Dipoena Thorell, 1869

Synonym: Genus Trigonobothrys Simon, 1889

Comments: The genus Trigonobothrys was synonymised with genus Dipoena by Yoshida (2002).

1. Dipoena ahenea (Dyal, 1935)

Synonym: Trigonobothrys aheneus Dyal, 1935

Comments: Endemic to Pakistan

2. Dipoena notata Dyal, 1935

Comments: Endemic to Pakistan

XXI.d. Genus Rhomphaea L. Koch, 1872

Comments: Removed from the synonym of genus Argyrodes by Agnarsson (2004).

1. Rhomphaea ornatissima Dyal, 1935 Comments: Endemic to Pakistan. Original name reinstated by Agnarsson (2004).

XXI.e. Genus Spintharus Hentz, 1850

1. Spintharus argenteus Dyal, 1935

Comments: Endemic to Pakistan.

\section{FAMILY THOMISIDAE SUNDEVALL, 1833}

XXII.a. Genus Diaea Thorell, 1869

1. Diaea expallidata (O.P.-Cambridge, 1885)

Comments: Endemic to Pakistan

2. Diaea subdola O.P.-Cambridge, 1885

Synonyms: Misumena yunohamensis Bösenberg \& Strand, 1906

Misumena japonica Bösenberg \& Strand, 1906

Misumenops japonicus (Bösenberg \& Strand, 1906)

Diaea japonicus (Bösenberg \& Strand, 1906)

Misumena horai Tikader, 1962

Distribution: Russia, India, Pakistan to Japan

Comments: Misumena horai was synonymised with

Diaea subdola by Marusik (1993).

3. Diaea terrena Dyal, 1935

Comments: Endemic to Pakistan

XXII.b. Genus Ebrechtella Dahl, 1907

1. Ebrechtella concinna (Thorell, 1877)

Synonym: Diaea concinna Thorell, 1877

Diaea subargentata O. P.-Cambridge, 1885

Misumena dierythra Thorell, 1892

Ebrechtella fruhstorferi Dahl, 1907

Misumenops direythra (Thorell, 1892)

Misumena gamma Chrysanthus, 1964

Misumena silveryi Tikader, 1965

Misumenops subargentatus (O. P.-Cambridge, 1885)

Misumenops maygitgitus Barrion \& Litsinger, 1995

Distribution: Pakistan to Philippines, Sulawesi, New Guinea

Comments: Diaea concinna was transferred to the genus Ebrechtella by Lehtinen (2005).

2. Ebrechtella sufflava (O.P.-Cambridge, 1885) Synonym: Diaea sufflava O.P.-Cambridge, 1885 Misumena expallidata O.P.-Cambridge, 1885

Comments: Endemic to Pakistan. Misumena expallidata was synonymised with Ebrechtella sufflava by Lehtinen (2005).
XXII.c. Genus Lysiteles Simon, 1895

1. Lysiteles excultus (O.P.-Cambridge, 1885)

Synonym: Synema exculta O.P.-Cambridge, 1885

Distribution: India, Pakistan

Comments: Endemic to South Asia. Synema exculta was transferred to the genus Lysiteles by Marusik (1993).

2. Lysiteles spinulosa (O.P.-Cambridge, 1885 Synonym: Diaea spinulosa O.P.-Cambridge, 1885 Distribution: Pakistan, India

Comments: Endemic to South Asia. Diaea spinulosa was transferred to the genus Lysiteles by Marusik (1993).

XXII.d. Genus Parastrophius Simon, 1903

1. Parastrophius vishwai Dyal, 1935

Comments: Endemic to Pakistan

XXII.e. Genus Runcinia Simon, 1875

1. Runcinia spinulosa (O.P.-Cambridge, 1885)

Synonym: Diaea spinulosa O.P.-Cambridge, 1885

Distribution: Pakistan, India

Comments: Endemic to South Asia. Diaea spinulosa

was transferred to the genus Runcinia by Marusik (1993).

XXII.f. Genus Tarrocanus Simon, 1895

Comments: This genus is endemic to South Asia.

1. Tarrocanus viridis Dyal, 1935

Comments: Endemic to Pakistan

XXII.g. Genus Thomisus Walckenaer, 1805

1. Thomisus albens O.P.-Cambridge, 1885

Distribution: Pakistan, Yarkand

2. Thomisus tuberculatus Dyal, 1935

Comments: Endemic to Pakistan

XXII.h. Genus Xysticus C.L. Koch, 1835

1. Xysticus setiger O.P.-Cambridge, 1885

Distribution: India, Pakistan

Comments: Endemic to South Asia

Pakistan spider summary

Number of families: 22

Number of Genera: 79

Number of Species: 138

Number of subspecies: 0

Number of Endemic Families: 0

Number of Endemic Genera: 0

Number of Endemic Species: 72

\section{Checklist 8. Spiders of Sri Lanka}

I. FAMILY AGELENIDAE C.L. KOCH, 1837

I.a. Genus Agelena Walckenaer, 1805

1. Tegenaria taprobanica Strand, 1907

Comments: Endemic to Sri Lanka

\section{FAMILY ARANEIDAE SIMON, 1895}

II.a. Genus Anepsion Strand, 1929

1. Anepsion maritatum (O.P.-Cambridge, 1877)

Synonyms: Paraplectana maritata O. P.-

Cambridge, 1877

Paraplectana picta Thorell, 1877

Paraplectana nigroanalis Hasselt, 1882

Anepsia maritata (O. P.-Cambridge, 1877)

Distribution: Sri Lanka, China to Sulawesi

II.b. Genus Araneus Clerck, 1757

1. Araneus enucleatus (Karsch, 1879)

Synonyms: Epeira enucleata Karsch, 1879

Epeira albertisii Thorell, 1887

Epeira soronis Thorell, 1890

Araneus soronis Simon, 1899

Distribution: India, Sri Lanka, Myanmar, Sumatra

2. Araneus obtusatus (Karsch, 1891)

Synonym: Epeira obtusata Karsch, 1891

Comments: Endemic to Sri Lanka.

II.c. Genus Argiope Audouin, 1826

1. Argiope taprobanica Thorell, 1887

Comments: Endemic to Sri Lanka

II.d. Genus Chorizopes O.P.-Cambridge, 1870 1. Chorizopes frontalis O.P.-Cambridge, 1870

Synonym: Chorizoopes frontalis O.P.-Cambridge, 1870

Distribution: Sri Lanka to Sumatra

Comments: Generic name misspelt in the orignal description.

2. Chorizopes mucronatus Simon, 1895

Comments: Endemic to Sri Lanka

II.e. Genus Cyrtarachne Thorell, 1868

1. Cyrtarachne perspicillata (Doleschall, 1859)

Synonyms: Epeira perspicillata Doleschall, 1859

Distribution: Sri Lanka, Sumatra, Java, New Guinea

2. Cyrtarachne raniceps Pocock, 1900

Distribution: India, Sri Lanka

Comments: Endemic to South Asia

II.f. Genus Cyrtophora Simon, 1864

1. Cyrtophora unicolor (Doleschall, 1857)

Synonyms: Epeira unicolor Doleschall, 1857

Epeira stigmatisata Karsch, 1878

Epeira stigmatisata serrata Thorell, 1890

Araneus unicolor Pocock, 1897

Distribution: Sri Lanka to Philippines, Australia

II.g. Genus Gasteracantha Sundevall, 1833

1. Gasteracantha geminata (Fabricius, 1798)

Synonyms: Aranea geminata Fabricius, 1798

Plectana geminata (Fabricius, 1798)

Gasteracantha connata Butler, 1873

Gasteracantha rimata O.P.-Cambridge, 1879

Distribution: India, Sri Lanka

Comments: Endemic to South Asia

2. Gasteracantha remifera Butler, 1873

Synonym: Plectana clavatrix Karsch, 1891

Distribution: India, Sri Lanka

Comments: Endemic to South Asia

II.h. Genus Glyptogona Simon, 1884

Comments: Endemic to Sri Lanka 
1. Glyptogona duriuscula Simon, 1895 Comments: Endemic to Sri Lanka

II.i. Genus Homalopoltys Simon, 1895 1. Homalopoltys albidus Simon, 1895 Comments: Endemic to Sri Lanka

2. Homalopoltys incanescens Simon, 1895 Comments: Endemic to Sri Lanka

II.j. Genus Hypsosinga Ausserer, 1871 1. Hypsosinga taprobanica (Simon, 1895) Synonym: Pronous taprobanicus Simon, 1895 Comments: Endemic to Sri Lanka. Pronous taprobanicus was transferred to genus Hypsosinga by Levi (1995).

II.k. Genus Mangora O. P.-Cambridge, 1889 1. Mangora semiargentea Simon, 1895 Comments: Endemic to Sri Lanka

II.I. Genus Ordgarius Keyserling, 1886 1. Ordgarius hobsoni (OP.-Cambridge, 1877) Synonym: Cyrtarachne hobsoni O.P.-Cambridge, 1877 Distribution: India, Sri Lanka, China, Japan

II.m. Gebus Poltys C.L. Koch, 1843

1. Poltys columnaris Thorell, 1890

Distribution: Sri Lanka, Sumatra

II.n. Gebus Ursa Simon, 1895

1. Ursa vittigera Simon, 1895

Comments: Endemic to Sri Lanka

\section{FAMILY BARYCHELIDAE SIMON, 1889}

III.a. Genus Diplothele O. P.-Cambridge, 1890

Comments: Endemic to South Asia

1. Diplothele halyi Simon, 1892

Comments: Endemic to Sri Lanka

III.b. Genus Plagiobothrus Karsch, 1891

Comments: This genus is monotypic and endemic to Sri Lanka.

1. Plagiobothrus semilunaris Karsch, 1891

Comments: Endemic to Sri Lanka

III.c. Genus Sason Simon, 1887

1. Sason robustum (O. P.-Cambridge, 1883)

Synonyms: Sarpedon robustum O. P.-Cambridge, 1883

Oecophlaeus cinctipes Pocock, 1892

Sason cinctipies (Pocock, 1892)

Sason armatoris Pocock, 1900

Distribution: India, Sri Lanka, Seychelles

Comments: Sason cinctipies and Sason armatoris were synonymised with Sason robustum by Raven (1986).

III.d. Genus Sipalolasma Simon, 1892

1. Sipalolasma ellioti Simon, 1892

Comments: Endemic to Sri Lanka

2. Sipalolasma greeni Pocock, 1900

Comments: Endemic to Sri Lanka

IV. FAMILY CLUBIONIDAE WAGNER, 1887

IV.a. Gebus Matidia Thorell, 1878

1. Matidia flagellifera Simon, 1897

Comments: Endemic to Sri Lanka

2. Matidia simplex Simon, 1897

Comments: Endemic to Sri Lanka

IV.b. Genus Nusatidia Deeleman-Reinhold, 2001

1. Nusatidia bimaculata (Simon, 1897)

Synonym: Matidia bimaculata Simon, 1897
Comments: Endemic to Sri Lanka. Matidia bimaculata was transferred to genus Nusatidia by DeelemanReinhold (2001).

V.c. Genus Simalio Simon, 1897

1. Simalio lucorum Simon, 1906

Comments: Endemic to Sri Lanka

2. Simalio phaeocephalus Simon, 1906

Comments: Endemic to Sri Lanka

\section{FAMILY CORINNIDAE KARSCH, 1880}

Comments: Considered valid by Wunderlich (1986), so genera listed by Brignoli under Castianeirinae and Corinninae (Clubionidae) are treated here and also genera listed by Roewer under the Corinninae have been apportioned to the Corinnidae (Platnick, 2006).

V.a. Genus Aetius O.P.-Cambridge, 1896 1. Aetius decollatus O.P.-Cambridge, 1896 Distribution: India, Sri Lanka

Comments: Endemic to South Asia

V.b. Genus Coenoptychus Simon, 1885

Comments: It is a monotypic genus and endemic to South Asia.

1. Coenoptychus pulcher Simon, 1885

Synonyms: Onychocryptus mutillaris Karsch, 1891 Coenoptychus pulchellus Simon, 1897

Distribution: India, Sri Lanka

Comments: Endemic to South Asia

V.c. Genus Copa Simon, 1885

1. Copa annulata Simon, 1896

Comments: Endemic to Sri Lanka

2. Copa spinosa Simon, 1896

Comments: Endemic to Sri Lanka

V.d. Genus Koppe Deeleman-Reinhold, 2001 1. Koppe armata (Simon, 1896)

Synonyms: Medmassa armata Simon, 1896 Comments: Endemic to Sri Lanka. DeelemanReinhold (2001) transferred Medmassa armata to genus Koppe.

V.e. Genus Oedignatha Thorell, 1881 1. Oedignatha affinis Simon, 1897 Comments: Endemic to Sri Lanka

2. Oedignatha bicolor Simon, 1896 Comments: Endemic to Sri Lanka

3. Oedignatha coriacea Simon, 1897 Comments: Endemic to Sri Lanka

4. Oedignatha flavipes Simon, 1897 Comments: Endemic to Sri Lanka

5. Oedignatha gulosa Simon, 1897 Comments: Endemic to Sri Lanka

6. Oedignatha major Simon, 1896 Comments: Endemic to Sri Lanka

7. Oedignatha montigena Simon, 1897

Comments: Endemic to Sri Lanka

8. Oedignatha proboscidea (Strand, 1913)

Comments: Endemic to Sri Lanka

9. Oedignatha retusa Simon, 1897 Comments: Endemic to Sri Lanka

10. Oedignatha striata Simon, 1897 Comments: Endemic to Sri Lanka
V.f. Genus Orthobula Simon, 1897 1. Orthobula impressa Simon, 1897 Distribution: Sri Lanka, Seychelles

V.g. Genus Sphecotypus O. P.-Cambridge, 1895 1. Sphecotypus taprobanicus Simon, 1897 Comments: Endemic to Sri Lanka

V.h. Genus Trachelas L. Koch, 1872 1. Trachelas oreophilus Simon, 1906 Synonyms: Trachelas oriophilus Simon, 1906 Trachelas oreophila Simon, 1906

Distribution: India, Sri Lanka

Comments: Endemic to South Asia

2. Trachelas quisquiliarum Simon, 1906

Comments: Endemic to Sri Lanka

V.i. Genus Utivarachna Kishida, 1940

1. Utivarachna accentuata (Simon, 1896)

Synonyms: Trachelas accentuatus Simon, 1896 Comments: Endemic to Sri Lanka. Trachelas accentuatus was transferred to genus Utivarachna by Deeleman-Reinhold (2001).

VI. FAMILY CRYPTOTHELIDAE L. KOCH, 1872 Comments: It is monotypic family.

VI.a. Genus Cryptothele L. Koch, 1872

Comments: This genus was previously considered in the family Zodariidae by Tikader (1987). Davies (1985) and Jocqué (1986a, b) placed it in the family Cryptothelidae.

1. Cryptothele ceylonica O. P.-Cambridge, 1877 Comments: Endemic to Sri Lanka

\section{FAMILY CTENIDAE KEYSERLING, 1877}

VII.a. Genus Ctenus Walckenaer, 1805

1. Ctenus ceylonensis F. O. P.-Cambridge, 1897

Synonym: Ctenus cuspidatus F. O. P.-Cambridge, 1902

Comments: Endemic to Sri Lanka. Ctenus

cuspidatus was synonimysed with Ctenus ceylonensis by Tikader \& Malhotra (1981).

2. Ctenus karschi Roewer, 1951

Synonym: Ctenus trabifer Karsch, 1891

Comments: Endemic to Sri Lanka. Ctenus trabifer was misidentified by Karsch (1891) and thus, Roewer (1951) provided a replacement name to Ctenus trabifer.

3. Ctenus thorelli F. O. P.-Cambridge, 1897

Synonym: Ctenus thorellii F. O. P.-Cambridge, 1897 Comments: Endemic to Sri Lanka

VII.b. Genus Diallomus Simon, 1897

Comments: Endemic to Sri Lanka

1. Diallomus fuliginosus Simon, 1897 Comments: Endemic to Sri Lanka

2. Diallomus speciosus Simon, 1897 Comments: Endemic to Sri Lanka

VIII. FAMILY DICTYNIDAE O.P.-CAMBRIDGE, 1871

VIII.a. Genus Anaxibia Thorell, 1898

1. Anaxibia nigricauda (Simon, 1905)

Synonym: Dictyna nigricauda Simon, 1905

Distribution: India, Sri Lanka

Comments: Endemic to South Asia. Dictyna nigricauda was transferred to genus Anaxibia by Lehtinen (1967). Sri Lanka was not included in distribution by Siliwal et al. (2005) 
VIII.b. Genus Atelolathys Simon, 1892 Comments: This genus is monotypic and endemic to Sri Lanka.

\section{Atelolathys varia Simon, 1892}

Comments: Endemic to Sri Lanka.

VIII.c. Genus Dictyna Sundevall, 1833

1. Dictyna turbida Simon, 1905

Synonyms: Dictyna kandiana Simon, 1906

Brigittea turbida Lehtinen, 1967

Distribution: India, Sri Lanka

Comments: Endemic to South Asia. Transfer of

Dictyna turbida to genus Brigittea was considered

incorrect and thus original name was reinstated by Platnick (2006)

VIII.d. Genus Dictynomorpha Spassky, 1939

1. Dictynomorpha smaragdula (Simon, 1905)

Synonym: Dictyna smaragdula Simon, 1905

Comments: Endemic to Sri Lanka. Dictyna smaragdula was transferred to genus Dictynomorpha by Lehtinen (1967).

VIII.e. Genus Rhion O. P.-Cambridge, 1870 Comments: Comments: This genus is monotypic genus and endemic to Sri Lanka.

1. Rhion pallidum O. P.-Cambridge, 1870

Comments: Endemic to Sri Lanka.

\section{FAMILY DIPLURIDAE SIMON, 1889}

IX.a. Genus Indothele Coyle, 1995

Comments: Endemic to South Asia.

1. Indothele lanka Coyle, 1995

Comments: Endemic to Sri Lanka

\section{FAMILY ERESIDAE C.L. KOCH, 1851}

X.a. Genus Stegodyphus Simon, 1873

1. Stegodyphus sarasinorum Karsch, 1891

Distribution: India, Sri Lanka, Nepal

Comments: Endemic to South Asia

\section{FAMILY HAHNIIDAE BERTKAU, 1878}

XI.a. Genus Alistra Thorell, 1894

1. Alistra radleyi (Simon, 1898)

Synonym: Aviola radleyi Simon, 1898

Comments: Endemic to Sri Lanka. According to 1. Scalidognathus oreophilus Simon, 1892

Platnick (2006), Aviola radleyi was transferred to genus Comments: Endemic to Sri Lanka

Alistra.

2. Alistra stenura (Simon, 1898)

Synonym: Aviola stenura Simon, 1898

Comments: Endemic to Sri Lanka. According to Platnick (2006), Aviola stenura was transferred to genus Alistra.

3. Alistra taprobanica (Simon, 1898)

Synonym: Hahnia taprobanica Simon, 1898

Comments: Endemic to Sri Lanka. Hahnia taprobanica was transferred to genus Alistra by Lehtinen (1967).

XI.b. Genus Hahnia C.L. Koch, 1841

1. Hahnia oreophila Simon, 1898

Synonym: Muizenbergia oreophila (Simon, 1898)

Comments: Endemic to Sri Lanka. Original name reinstated by (Platnick, 2006).

2. Hahnia pusio Simon, 1898

Comments: Endemic to Sri Lanka

XII. FAMILY HERSILIIDAE THORELL, 1870

XII.a. Genus Hersilia Audouin, 1826
1. Hersilia savignyi Lucas, 1836

Synonyms: Hersilia indica Walckenaer, 1837

Hersilia calcuttensis Stoliczka, 1869

Hersilia clathrata Thorell, 1895

Distribution: Sri Lanka, India to Philippines

Comments: Hersilia clathrata was synonymised with

Hersilia savignyi by Baehr \& Baehr (1993).

2. Hersilia tibialis Baehr \& Baehr, 1993

Synonym: Hersilia pectinata Thorell, 1895 (in

Sinha, 1951)

Distribution: India, Sri Lanka

Comments: Endemic to South Asia. According to

Platnick (2006) Hersilia pectinata described by

Sinha (1951) from India was a misidentification.

XII.b. Genus Murricia Simon, 1882

1. Murricia crinifera Baehr \& Baehr, 1993

Comment: Endemic to Sri Lanka

XII.c. Genus Neotama Baehr \& Baehr, 1993

1. Neotama variata (Pocock, 1899)

Synonym: Tama variata Pocock, 1899

Comments: Endemic to Sri Lanka. Tama variata was

ransferred to genus Neotama by Baehr \& Baehr

(1993).

XII.d. Genus Promurricia Baehr \& Baehr, 1993

Comments: This is monotypic genus and endemic to Sri Lanka.

1. Promurricia depressa Baehr \& Baehr, 1993

Comments: Endemic to Sri Lanka

\section{FAMILY IDIOPIDAE SIMON, 1892}

XIII.a. Genus Heligmomerus Simon, 1892

Comments: Raven (1985) transferred genus Heligmomerus from family Ctenizidae to family Idiopidae.

1. Heligmomerus taprobanicus Simon, 1892

Comments: Endemic to Sri Lanka

XIII.b. Genus Scalidognathus Karsch, 1891 Synonym: Genus Nemesiellus Pocock, 1900 Comments: Scalidognathus was transferred from Ctenizidae to Idiopidae and was considered a senior synonym of Nemesiellus Pocock, 1900 by Raven (1985)

2. Scalidognathus radialis (O.P.-Cambridge, 1869)

Synonym: Mygale radialis O.P.-Cambridge, 1869

Scalidognathus seticeps Karsch, 1891

Comments: Endemic to Sri Lanka

XIV. FAMILY LINYPHIIDAE BLACKWALL, 1859

XIV.a. Genus Atypena Simon, 1894

1. Atypena ellioti Jocqué, 1983

Comments: Endemic to Sri Lanka

2. Atypena simoni Jocqué, 1983

Comments: Endemic to Sri Lanka

XIV.b. Genus Ceratinopsis Emerton, 1882

1. Ceratinopsis monticola (Simon, 1894)

Synonym: Lygarina monticola Simon, 1894

Comments: Endemic to Sri Lanka. Lygarina monticola was transferred to genus Ceratinopsis by Millidge (1995).

XIV.c. Genus Helsdingenia Saaristo \& Tanasevitch, 2003 1. Helsdingenia ceylonica (van Helsdingen, 1985) Distribution: Nepal, Sri Lanka
Comments: Endemic to South Asia

XIV.d. Genus Labullinyphia van Helsdingen, 1985 Comments: It is a monotypic genus and endemic to Sri Lanka

1. Labullinyphia tersa (Simon, 1894)

Synonym: Linyphia tersa Simon, 1894a

Comments: Endemic to Sri Lanka. Linyphia tersa was transferred to genus Labullinyphia by van Helsdingen (1985).

XIV.e. Genus Microbathyphantes van Helsdingen, 1985 1. Microbathyphantes palmarius (Marples, 1955)

Synonyms: Linyphia palmaria Marples, 1955 Microbathyphantes asiaticus van Helsdingen, 1985

Priscipalpus palmarius (Marples, 1955)

Distribution: Sri Lanka, Seychelles, Myanmar, Polynesia

Comments: Priscipalpus palmarius was transferred to genus Microbathyphantes and Microbathyphantes asiaticus was synonymised with Microbathyphantes palmarius by Saaristo (1995)

XIV.f. Genus Nematogmus Simon, 1884

1. Nematogmus dentimanus Simon, 1886

Synonym: Nematogmus dentimanus Simon, 1886

Linyphia javana Workman, 1896

Sphecozone dentimanus (Simon, 1886)

Distribution: Sri Lanka to Malaysia, Java, Krakatau

Comments: Original name reinstated by van

Helsdingen (1979)

XIV.g. Genus Neriene Blackwall, 1833

1. Neriene katyae van Helsdingen, 1969

Comments: Endemic to Sri Lanka

XIV.h. Genus Nesioneta Millidge, 1991

1. Nesioneta benoiti (van Helsdingen, 1978)

Synonym: Meioneta benoiti van Helsdingen, 1978

Lepthyphantes brincki van Helsdingen, 1985

Distribution: Sri Lanka, Seychelles

Comments: Meioneta benoiti was transferred to genus Nesioneta and Lepthyphantes brincki was synonymised with Nesioneta benoiti by Saaristo (1995).

XIV.i. Genus Obrimona Strand, 1934

Synonym: Genus Obrima Simon, 1894

Comments: This is monotypic genus and endemic to Sri Lanka. Generic name Obrima was preoccupied and thus, Strand (1934) provided replacement name.

1. Obrimona tennenti (Simon, 1894)

Comments: Endemic to Sri Lanka

XIV.j. Genus Trematocephalus Dahl, 1886

1. Trematocephalus simplex Simon, 1894

Comments: Endemic to Sri Lanka.

2. Trematocephalus tripunctatus Simon, 1894

Comments: Endemic to Sri Lanka.

XIV.k. Genus Typhistes Simon, 1894

1. Typhistes antilope Simon, 1894

Comments: Endemic to Sri Lanka.

2. Typhistes comatus Simon, 1894

Comments: Endemic to Sri Lanka.

\section{FAMILY LIOCRANIDAE SIMON, 1897}

XV.a. Genus Argistes Simon, 1897

1. Argistes seriatus (Karsch, 1891)

Synonym: Leptodrassus seriatus Karsch, 1891

Comments: Endemic to Sri Lanka. According to Platnick (2006), Leptodrassus seriatus was transferred to genus Argistes. 
2. Argistes velox Simon, 1897

Comments: Endemic to Sri Lanka

XV.b. Genus Paratus Simon, 1898

Comments: This is a monotypic genus and is endemic to Sri Lanka.

\section{Paratus reticulatus Simon, 1898} Comments: Endemic to Sri Lanka

XV.c. Genus Sphingius Thorell, 1890

Comments: This genus was originally described in 2. Pardosa palliclava (Strand, 1907) the family Clubionidae. After a series of transfers, it Synonym: Lycosa palliclava Strand, 1907

was finally transferred to family Liocranidae by Comments: Endemic to Sri Lanka Deeleman-Reinhold (2001).

1. Sphingius scutatus Simon, 1897 Comments: Endemic to Sri Lanka

\section{FAMILY LYCOSIDAE SUNDEVALL, 1833}

XVI.a. Genus Hippasa Simon, 1885

1. Hippasa greenalliae (Blackwall, 1867)

Synonyms: Lycosa greenalliae Blackwall, 1867

Hippasa lingxianensis Yin \& Wang, 1980

Hippasa pantherina Pocock, 1899

Distribution: India, Sri Lanka, China

Comments: Hippasa pantherina was synonymised with Hippasa greenalliae by Tikader \& Malhotra (1980).

XVI.b. Genus Hogna Simon, 1885

Synonym: Genus Lycorma Simon, 1885

Comments: The genus Lycorma was synonymised with the genus Hogna by Wunderlich (1992).

1. Hogna lupina (Karsch, 1879)

Synonyms: Lycosa lupina Karsch, 1879

Sschizocosa lupina (Karsch, 1879)

Comments: Endemic to Sri Lanka. Sschizocosa

lupina was transferred to genus Hogna by Roewer (1959).

XVI.c. Genus Lycosa Latreille, 1804 Synonym: Genus Allohogna Roewer, 1955 Comments: Allohogna was synonymised with genus Lycosa by Fuhn \& Niculescu-Burlacu (1971).

1. Lycosa indagatrix Walckenaer, 1837

Synonyms: Leimonia indagatrix (Walckenaer, 1837)

Hogna catula Reimoser, 1934

Hogna indagatrix (Walckenaer, 1837)

Lycosa catula (Reimoser, 1934)

Distribution: India, Sri Lanka

Comments: Endemic to South Asia. According to Platnick (2006) Lycosa catula was synonymised with Lycosa indagatrix. Transfer of Lycosa indagatrix to genus Hogna was considered incorrect and thus original name was reinstated by Tikader and Malhotra (1980).

2. Lycosa yerburyi Pocock, 1901

Synonym: Hogna yerburyi (Pocock, 1901)

Lycosa yerburgi (Pocock, 1901)

Comments: Endemic to Sri Lanka. Tikader \& Malhotra

(1980) considered transfer of Lycosa yerburyi to genus Hogna invalid. Original name reinstated.

XVI.d. Genus Ocyale Audouin, 1826

1. Ocyale lanca (Karsch, 1879)

Synonym: Lycosa lanca Karsch, 1879

Comments: Endemic to Sri Lanka. Lycosa lanca was

transferred to genus Ocyale by Roewer (1955).

2. Ocyale pilosa (Roewer, 1960)

Synonym: Dolomedes ocyale Walckenaer, 1837

Ocyale ocyale (Walckenaer, 1837)

Trochosa lactea L. Koch, 1875

Ocyale atalanta Simon, 1885

Hippasosa pilosa Roewer, 1960

Ocyale neatalanta Alderweireldt, 1996

Distribution: West Africa to Myanmar

XVI.e. Genus Pardosa C. L. Koch, 1847

1. Pardosa leucopalpis Gravely, 1924

Distribution: India, Pakistan, Sri Lanka

Comments: Endemic to South Asia

3. Pardosa semicana Simon, 1885

Pardosa subsemicana Schenkel, 1963

4. Pardosa timidula (Roewer, 1951)

Synonyms: Lycosa timida Simon, 1882

Pardosa timida (Simon, 1882)

Lycosa timidula Roewer, 1951

Hogna timidula (Roewer, 1951)

Allocosa timidula (Roewer, 1951)

Pardosa timida (Simon, 1882)

Distribution: Yemen, Sri Lanka, Pakistan

XVI.f. Genus Wadicosa Zyuzin, 1985

1. Wadicosa quadrifera (Gravely, 1924)

Pardosa quadrifera (Gravely, 1924)

Lycosa quadrifer (Gravely, 1924)

Wadicosa quadrifer (Gravely, 1924)

Distribution: India, Sri Lanka Kronestedt (1993).

XVI.g. Genus Zoica Simon, 1898

1. Zoica parvula (Thorell, 1895)

Synonym: Zobia parvula Thorell, 1895

Malaysia

2. Zoica puellula (Simon, 1898)

Synonym: Flanona puellula Simon, 1898

Distribution: India, Sri Lanka

Comments: Endemic to South Asia

XVII. FAMILY MIMETIDAE SIMON, 1881

XVII.a. Genus Mimetus Hentz, 1832

1. Mimetus strinatii Brignoli, 1972

Comments: Endemic to Sri Lanka

XVII.b. Genus Phobetinus Simon, 1895

1. Phobetinus sagittifer Simon, 1895

Comments: Endemic to Sri Lanka Brescovit (1997).
Comments: Ocyale neatalanta was synonymised 2. Cheiracanthium indicum O.P.-Cambridge, 1874

with Ocyale pilosa by Alderweireldt \& Jocqué (2005). Distribution: India, Sri Lanka

Synonym: Lycosa subsemicana Strand, 1909

Distribution: Sri Lanka, Malaysia, China

Comments: The species name Lycosa timida was

preoccupied by Lucas (1846) and thus Roewer

(1951) provided replacement name Lycosa timidula for Lycosa timida. Allocosa timida was transferred to genus Pardosa by Tikader and Malhotra (1980).

Synonyms: Lycosa quadrifera Gravely, 1924

Comments: Endemic to South Asia. Lycosa

quadrifera was transferred to genus Wadicosa by

Comments: Considered a senior synonym of genus

Flanona Simon, 1898 by Lehtinen \& Hippa (1979).

Distribution: Sri Lanka, Myanmar, Thailand,

Comments: Zoica is a replacement name for

genus Zobia, as genus name was preoccupied.

XVIII. FAMILY MITURGIDAE SIMON, 1885

XVIII.a. Genus Cheiracanthium C. L. Koch, 1839

Comments: Genus Cheiracanthium (Clubionidae) was transferred to family Miturgidae by Bonaldo \&
1. Cheiracanthium incertum O.P.-Cambridge, 1869 Comments: Endemic to Sri Lanka

Comments: Endemic to South Asia

3. Cheiracanthium insigne O.P.-Cambridge, 1874 Distribution: India, Sri Lanka, China

4. Cheiracanthium taprobanense Strand, 1907

XIX. FAMILY MYSMENIDAE PETRUNKEVITCH, 1928

XIX.a. Genus Mysmenella Brignoli, 1980

1. Mysmenella saltuensis (Simon, 1895)

Comments: Endemic to Sri Lanka

XIX.b. Genus Phricotelus Simon, 1895

Comments: This genus is monotypic and endemic to Sri Lanka.

1. Phricotelus stelliger Simon, 1895

Comments: Endemic to Sri Lanka

\section{FAMILY NEPHILIDAE SIMON, 1894}

Comments: Subfamily Nephilinae under family Kuntner (2006). Genera Clitaetra, Herennia, Nephila and Nephilengys were transferred from family Tetragnathidae to this family.

XX.a. Genus Clitaetra Simon, 1889

1. Clitaetra thisbe Simon, 1903

Comments: Endemic to Sri Lanka

XX.b. Genus Herennia Thorell, 1877

1. Herennia multipuncta (Doleschall, 1859)

Synonyms: Epeira ornatissima Doleschall, 1859

Epeira mammillaris Stoliczka, 1869

Herennia sampitana Karsch, 1880

Herennia mollis Thorell, 1887

Herennia ornatissima (Doleschall, 1859)

Distribution: India to China, Sri Lanka, Malaysia,

New Guinea

Comments: Herennia ornatissima was synonymised with Herennia multipuncta by Kuntner (2005).

XX.c. Genus Nephila Leach, 1815

1. Nephila pilipes (Fabricius, 1793)

Synonyms: Aranea longipes Fabricius 1781

Aranea maculata Fabricius, 1793

Aranea pilipes Fabricius, 1793

Aranea sebae Walckenaer, 1802

Epeira chrysogaster Walckenaer, 1805

Nephila maculata (Fabricius, 1793)

Nephila fuscipes C.L. Koch, 1839

Epeira fuscipes (C.L. Koch, 1839)

Epeira doreyana Walckenaer, 1842

Epeira caliginosa Walckenaer, 1842

Nephila ornata Adams, 1847

Epeira chrysogaster (Walckenaer, 1805)

Epeira harpyia Doleschall, 1859

Nephila chrysogaster (Walckenaer, 1805)

Meta ornata (Adams, 1847)

Nephila pecuniosa L. Koch, 1872

Nephila aurosa L. Koch, 1872

Nephila procera L. Koch, 1872

Nephila sulphurosa L. Koch, 1872

Nephila tenuipes L. Koch, 1872

Nephila submaculata Strand, 1906

Distribution: India, Sri Lanka, China, Philippines to

Australia

Comments: According to Platnick (2006), Nephila

maculata was synonymised with Nephila pilipes.
Comments: Endemic to Sri Lanka Tetragnathidae was elevated to family level by

Epeira penicillum Doleschall, 1857 
XX.d. Genus Nephilengys L. Koch, 1872

1. Nephilengys malabarensis (Walckenaer, 1842)

Synonym: Metepeira andamanensis Tikader, 1977

Distribution: India, Sri Lanka to Philippines, Australia

Comments: Metepeira andamanensis was synonymised with Nephilengys malabarensis by Tikader (1982a).

\section{FAMILY NESTICIDAE Simon, 1894}

XXI.a. Genus Nesticella Lehtinen \& Saaristo, 1980 1. Nesticella aelleni (Brignoli, 1972)

Synonym: Nesticus aelleni Brignoli, 1972

Comments: Endemic to Sri Lanka. Nesticus aelleniwas

was transferred to genus Nesticella by Lehtinen \& Saaristo (1980)

\section{FAMILY OCHYROCERATIDAE FAGE, 1912}

XXII.a. Genus Merizocera Fage, 1912

1. Merizocera brincki Brignoli, 1975

Comments: Endemic to Sri Lanka

2. Merizocera cruciata (Simon, 1893)

Synonym: Ochyrocera cruciata Simon, 1893

Comments: Endemic to Sri Lanka.

3. Merizocera oryzae Brignoli, 1975

Comments: Endemic to Sri Lanka

4. Merizocera picturata (Simon, 1893)

Synonym: Ochyrocera picturata Simon, 1893

Comments: Endemic to Sri Lanka

XXII.b. Genus Psiloderces Simon, 1892

1. Psiloderces elasticus (Brignoli, 1975)

Synonym: Merizocera elastica Brignoli, 1975

Comments: Endemic to Sri Lanka. Merizocera elastica

was transferred to genus Psiloderces by Deeleman-

Reinhold (1995).

XXII.c. Genus Speocera Berland, 1914

1. Speocera taprobanica Brignoli, 1981

Synonym: Simonicera taprobanica Brignoli (1981) Comments: Endemic to Sri Lanka

Comments: Endemic to Sri Lanka. Original name

reinstated by Brignoli (1986).

\section{FAMILY OONOPIDAE SIMON, 1890}

XXIII.a. Genus Aprusia Simon, 1893

Comments: This genus is monotypic and endemic to

Sri Lanka.

\section{Aprusia strenuus Simon, 1893}

Comments: Endemic to Sri Lanka

XXIII.b. Genus Epectris Simon, 1893

1. Epectris mollis Simon, 1907

Comments: Endemic to Sri Lanka

XXIII.c. Genus Gamasomorpha Karsch, 1881

1. Gamasomorpha microps Simon, 1907

Comments: Endemic to Sri Lanka

2. Gamasomorpha nigripalpis Simon, 1893

Distribution: India, Sri Lanka

Comments: Endemic to South Asia

3. Gamasomorpha subclathrata Simon, 1907

Comments: Endemic to Sri Lanka

4. Gamasomorpha taprobanica Simon, 1893

Distribution: India, Sri Lanka

Comments: Endemic to South Asia

XXIII.d. Genus Ischnothyreus Simon, 1893

1. Ischnothyreus bipartitus Simon, 1893

Comments: Endemic to Sri Lanka
2. Ischnothyreus lymphaseus Simon, 1893

Comments: Endemic to Sri Lanka

3. Ischnothyreus vestigator Simon, 1893

Comments: Endemic to Sri Lanka

XXIII.e. Genus Opopaea Simon, 1891

1. Opopaea ambigua Simon, 1893

Comments: Endemic to Sri Lanka

XXIII.f. Genus Orchestina Simon, 1882

1. Orchestina dentifera Simon, 1893

Comments: Endemic to Sri Lanka

2. Orchestina manicata Simon, 1893

Distribution: Yemen, Sri Lanka, Vietnam

3. Orchestina pilifera Dalmas, 1916

Comments: Endemic to Sri Lanka

4. Orchestina tubifera Simon, 1893

Comments: Endemic to Sri Lanka

XXIII.i. Genus Xestaspis Simon, 1884

1. Xestaspis sublaevis Simon, 1893

Comments: Endemic to Sri Lanka

XXIV. FAMILY OXYOPIDAE THORELL, 1870

XXIV.a. Genus Oxyopes Latreille, 1804

1. Oxyopes ceylonicus Karsch, 1891

Comments: Endemic to Sri Lanka

2. Oxyopes daksina Sherriffs, 1955

Synonym: Oxyopes daksima Sherriffs, 1955

Distribution: Sri Lanka, China

3. Oxyopes hindostanicus Pocock, 1901

Distribution: India, Sri Lanka, Pakistan

Comments: Endemic to South Asia. Pakistan was not included in distribution by Siliwal et al. (2005).

4. Oxyopes juvencus Strand, 1907

5. Oxyopes nilgiricus Sherriffs, 1955

Comments: Endemic to Sri Lanka

6. Oxyopes rufisternis Pocock, 1901

Comments: Endemic to Sri Lanka

XXV. FAMILY PALPIMANIDAE THORELL, 1870

XXV.a. Genus Steriphopus Simon, 1887

1. Steriphopus macleayi (O.P.-Cambridge, 1873 )

Synonym: Pachypus macleayi O. P.-Cambridge, 1873

Comments: Endemic to Sri Lanka. The genus name

Pachypus was preocuppied and thus, Simon (1887)

provided replacement name Steriphopus for genus

Pachypus.

XXVI. FAMILY PHILODROMIDAE THORELL, 1870

XXVI.a. Genus Gephyrota Strand, 1932

1. Gephyrota virescens (Simon, 1906)

Synonym: Gephyra virescens Simon, 1906

Comments: Endemic to Sri Lanka

XXVI.b. Genus Tibellus Simon, 1875

Comments: Genus Tibellus was removed from

family Thomisidae and placed in the family

Philodromidae by Homann (1975).

1. Tibellus vitilis Simon, 1906

Distribution: India, Sri Lanka

Comments: Endemic to South Asia
XXVII. FAMILY PHOLCIDAE C.L. KOCH, 1851

XXVII.a. Genus Belisana Thorell, 1898

1. Belisana benjamini Huber, 2005

Comments: Endemic to Sri Lanka.

2. Belisana keyti Huber, 2005

Comments: Endemic to Sri Lanka.

3. Belisana ratnapura Huber, 2005

Comments: Endemic to Sri Lanka.

XXVII.b. Genus Holocneminus Berland, 1942

1. Holocneminus multiguttatus (Simon, 1905)

Synonym: Psilochorus multiguttatus Simon, 1905

Distribution: Sri Lanka to Malaysia, Sulawesi

Comments: Psilochorus multiguttatus was transferred

to genus Holocneminus by Deeleman-Reinhold (1995).

XXVII.c. Genus Pholcus Walckenaer, 1805

1. Pholcus ceylonicus O. P.-Cambridge, 1869

Comments: Endemic to Sri Lanka

2. Pholcus fragillimus Strand, 1907

Comments: Endemic to Sri Lanka

3. Pholcus quinquenotatus Thorell, 1878

Synonym: Pholcus v-notatus Thorell, 1878

Distribution: Sri Lanka, Amboina

XXVII.d. Genus Wanniyala Huber \& Benjamin, 2005 Comment: This genus is endemic to Sri Lanka.

1. Wanniyala agrabopath Huber \& Benjamin, 2005

Comments: Endemic to Sri Lanka

2. Wanniyala hakgala Huber \& Benjamin, 2005

Comments: Endemic to Sri Lanka

XXVIII. FAMILY PISAURIDAE SIMON, 1890

XXVIII.a. Genus Dolomedes Latreille, 1804

1. Dolomedes boiei (Doleschall, 1859)

Synonym: Lycosa boiei Doleschall, 1859

Tarantuloides boiei (Doleschall, 1859)

Distribution: Sri Lanka, Java

2. Dolomedes karschi Strand, 1913

Distribution: Endemic to Sri Lanka

XXVIII.b. Genus Perenethis L. Koch, 1878

1. Perenethis sindica (Simon, 1897)

Synonym: Tetragonophthalma sindica Simon, 1897

Perenethis indica Simon, 1897

Distribution: India, Sri Lanka, Nepal, China, Philippines

XXIX. FAMILY PSECHRIDAE SIMON, 1890

XXIX.a. Genus Psechrus Thorell, 1878

1. Psechrus torvus (O.P.-Cambridge, 1869)

Synonyms: Tegenaria torva O.P.-Cambridge, 1869

Lancaria torva (O.P.-Cambridge, 1869)

Psechrus alticeps Pocock, 1899

Distribution: Sri Lanka, India, China, Taiwan

Comments: Psechrus alticeps was synonymised

with Psechrus torvus by Lehtinen (1967).

XXX. FAMILY SALTICIDAE BLACKWALL, 1841

XXX.a. Genus Aelurillus Simon, 1884

1. Aelurillus kronestedti Azarkina, 2004

Comments: Endemic to Sri Lanka

2. Aelurillus quadrimaculatus Simon, 1889

Distribution: India, Sri Lanka

Comments: Endemic to South Asia

XXX.b. Genus Asemonea O.P.-Cambridge, 1869

1. Asemonea tenuipes (O.P.-Cambridge, 1869) 
Synonyms: Lyssomanes tenuipes O.P.-Cambridge, 1869

Asemonea cingulata Thorell, 1895

Lyssomanes andamanensis Tikader, 1977

Lyssomanes bengalensis Tikader \& Biswas, 1978

Distribution: India, Sri Lanka to Thailand

Comments: Lyssomanes bengalensis and

Lyssomanes andamanensis were synonymised with

Asemonea tenuipes by Wanless (1980).

XXX.c. Genus Ballus C.L. Koch, 1850

1. Ballus segmentatus Simon, 1900

Comments: Endemic to Sri Lanka

2. Ballus sellatus Simon, 1900

Comments: Endemic to Sri Lanka

XXX.d. Genus Bianor Peckham \& Peckham, 1886

1. Bianor angulosus (Karsch, 1879)

Synonyms: Ballus angulosus Karsch, 1879

Ballus trepidans Thorell, 1895

Simaetha angulosa (Karsch, 1879)

Ballus hotingchiehi Schenkel, 1963

Distribution: India, Sri Lanka, Thailand, Vietnam

XXX.e. Genus Brettus Thorell, 1895

1. Brettus adonis Simon, 1900

Synonym: Portia adonis Simon, 1901

Comments: Endemic to Sri Lanka. Oringinal name reinstated by Wanless (1979).

XXX.f. Genus Carrhotus Thorell, 1891

1. Carrhotus taprobanicus Simon, 1902

Comments: Endemic to Sri Lanka

XXX.g. Genus Colaxes Simon, 1900

Comments: Endemic to South Asia

1. Colaxes horton Benjamin, 2004

Comments: Endemic to Sri Lanka

2. Colaxes wanlessi Benjamin, 2004

Comments: Endemic to Sri Lanka

XXX.h. Genus Cosmophasis Simon, 1901

1. Cosmophasis olorina (Simon, 1901)

Synonym: Telamonia olorina Simon, 1901

Comments: Endemic to Sri Lanka. Telamonia olorina

was transferred to genus Cosmophasis by Prószyn'ski (1984).

XXX.i. Genus Curubis Simon, 1902

Comments: Endemic to South Asia.

1. Curubis annulata Simon, 1902

Comments: Endemic to Sri Lanka

2. Curubis erratica Simon, 1902

Comments: Endemic to Sri Lanka

3. Curubis tetrica Simon, 1902

Comments: Endemic to Sri Lanka

XXX.j. Genus Epidelaxia Simon, 1902

Comments: This genus is endemic to Sri Lanka.

1. Epidelaxia albocruciata Simon, 1902

Comments: Endemic to Sri Lanka

2. Epidelaxia albostellata Simon, 1902

Comments: Endemic to Sri Lanka

3. Epidelaxia obscura Simon, 1902

Comments: Endemic to Sri Lanka

XXX.k. Genus Euryattus Thorell, 1881

1. Euryattus bleekeri (Doleschall, 1859)

Synonym: Salticus bleekeri Doleschall, 1859
Plexippus bleekeri Thorell, 1878

Hasarius albescens Keyserling, 1881

Hasarius pauperatus Keyserling, 1881

Hasarius chrysostomus Keyserling, 1881

Hasarius pumilio Keyserling, 1881

Plotius curtus Simon, 1902

Euryattus albescens Simon, 1903

Plotius chrysostomus Simon, 1903

Plotius curtus Prószyn'ski, 1987

Distribution: Sri Lanka to Queensland

2. Euryattus breviusculus (Simon, 1902)

Synonym: Plotius breviusculus Simon, 1902

Comments: Endemic to Sri Lanka

XXX.I. Genus Evarcha Simon, 1902

1. Evarcha cancellata (Simon, 1902)

Synonym: Colopsus cancellatus Simon, 1902

Distribution: Sri Lanka, Java

Comments: Colopsus cancellatus was transferred to

genus Evarcha by Prószyn'ski (1984)

XXX.m. Genus Flacillula Strand, 1932

1. Flacillula lubrica (Simon, 1901)

Synonym: Flacilla lubrica Simon, 1901

Comments: Endemic to Sri Lanka

XXX.n. Genus Gelotia Thorell, 1890

1. Gelotia lanka Wijesinghe, 1991

Comments: Endemic to Sri Lanka

XXX.o. Genus Hispo Simon, 1885

1. Hispo bipartita Simon, 1903

Distribution: India, Sri Lanka

Comments: Endemic to South Asia

XXX.p. Genus Hyllus C.L. Koch, 1846

1. Hyllus semicupreus (Simon, 1885)

Synonyms: Thyene semicuprea Simon, 1885

Sandalodes semicupreus (Simon, 1885)

Phidippus indicus Tikader, 1974

Hyllus indicus (Tikader, 1974)

Distribution: India, Sri Lanka

Comments: Endemic to South Asia. Phidippus indicus

was transferred to genus Hyllus by Zabka (1988) and

synonymised with Hyllus semicupreus by Prószyn'sk

(1990); According to Platnick (2006) Sandalodes

semicupreus is transferred to genus Hyllus.

XXX.q. Genus Irura Peckham \& Peckham, 1901

1. Irura pulchra Peckham \& Peckham, 1901

Comments: Endemic to Sri Lanka

XXX.r. Genus Marengo Peckham \& Peckham, 1892

1. Marengo crassipes Peckham \& Peckham, 1892

Comments: Endemic to Sri Lanka

2. Marengo inornata (Simon, 1900)

Synonym: Philates inornatus Simon, 1900

Comments: Endemic to Sri Lanka. Philates inornatus was transferred to genus Marengo by Wanless (1978).

3. Marengo nitida Simon, 1900

Comments: Endemic to Sri Lanka

4. Marengo rattotensis Benjamin, 2006

Comments: Endemic to Sri Lanka

5. Marengo striatipes Simon, 1900

Comments: Endemic to Sri Lanka

XXX.s. Genus Modunda Simon, 1901

1. Modunda aeneiceps Simon, 1901

Synonym: Bianor aeneiceps (Simon, 1901)

Distribution: Sri Lanka, China

Comments: Original name reinstated by Logunov (2001).
XXX.t. Genus Myrmarachne MacLeay, 1839

1. Myrmarachne bicurvata (O.P.-Cambridge, 1869)

Synonyms: Salticus bicurvatus O.P.-Cambridge, 1869

Distribution: Endemic to Sri Lanka

2. Myrmarachne imbellis (Peckham \& Peckham, 1892)

Synonyms: Salticus imbellis Peckham \& Peckham,

1892

Distribution: Endemic to Sri Lanka

3. Myrmarachne plataleoides (O.P.-Cambridge, 1869) Synonyms: Salticus plataleoides O.P.-Cambridge, 1869 Myrmarachne daitarensis Prószyn'ski, 1992

Distribution: India, Sri Lanka, China, Southeast Asia

Comments: Myrmarachne daitarensis was

synonymised with Myrmarachne plataleoides by

Edmunds \& Prószyn'ski (2003).

4. Myrmarachne providens (Peckham \& Peckham,

1892

Synonym: Salticus providens Peckham \&

Peckham, 1892

Distribution: India, Sri Lanka

Comments: Endemic to South Asia

5. Myrmarachne spissa (Peckham \& Peckham, 1892) Synonym: Salticus spissus Peckham \& Peckham, 1892 Comments: Endemic to Sri Lanka

XXX.u. Genus Onomastus Simon, 1900

1. Onomastus nigricaudus Simon, 1900

Comments: Endemic to Sri Lanka

2. Onomastus quinquenotatus Simon, 1900

Comments: Endemic to Sri Lanka

XXX.v. Genus Panachraesta Simon, 1900

Comments: This is monotypic genus and endemic to Sri Lanka.

1. Panachraesta paludosa Simon, 1900

Comments: Endemic to Sri Lanka

XXX.w. Genus Panysinus Simon, 1901

1. Panysinus semiermis Simon, 1902

Comments: Endemic to Sri Lanka

XXX.x. Genus Phaeacius Simon, 1900

1. Phaeacius wanlessi Wijesinghe, 1991

Distribution: Nepal, Sri Lanka

Comments: Endemic to South Asia

XXX.y. Genus Phausina Simon, 1902

1. Phausina bivittata Simon, 1902

Comments: Endemic to Sri Lanka

2. Phausina flavofrenata Simon, 1902

Comments: Endemic to Sri Lanka

3. Phausina guttipes Simon, 1902

Comments: Endemic to Sri Lanka

XXX.z. Genus Phintella Strand, 1906

1. Phintella multimaculata (Simon, 1901)

Synonym: Chrysilla multimaculata Simon, 1901

Comments: Endemic to Sri Lanka. Chrysilla multimaculata was transferred to the genus Phintella by Prószyn'ski (1984)

2. Phintella volupe (Karsch, 1879)

Synonym: Attus volupe Karsch, 1879

Distribution: Sri Lanka, Bhutan

Comments: Endemic to South Asia. Attus volupe was transferred to genus Phintella by Zabka (1988).

XXX.aa. Genus Phyaces Simon, 1902

Comments: This genus is monotypic and endemic to Sri Lanka. 
1. Phyaces comosus Simon, 1902 Comments: Endemic to Sri Lanka

XXX.ab. Genus Plexippus C.L. Koch, 1846

1. Plexippus redimitus Simon, 1902

Distribution: India, Sri Lanka

Comments: Endemic to South Asia

XXX.ac. Genus Portia Karsch, 1878

Synonym: Genus Linus Peckham \& Peckham, 1885 Comment: Genus Linus was synonymised with genus Portia by Wanless (1978).

1. Portia fimbriata (Doleschall, 1859)

Synonym: Salticus fimbriatus Doleschall, 1859

Sinis fimbriatus (Doleschall, 1859)

Linus fimbriatus (Doleschall, 1859)

Linus alticeps Pocock, 1899

Boethoportia ocellata Hogg, 1915

Distribution: Nepal, Sri Lanka, Taiwan to Australia

2. Portia labiata (Thorell, 1887)

Synonym: Sinis fimbriatus Hasselt, 1882

Linus labiatus Thorell, 1887

Linus dentipalpis Thorell, 1890

Erasinus dentipalpis (Thorell, 1890)

Erasinus labiatus (Thorell, 1887)

Distribution: Sri Lanka to Philippines

Comments: Erasinus labiatus was transferred to the genus Portia by Wanless (1978).

XXX.ad. Genus Ptocasius Simon, 1885

1. Ptocasius fulvonitens Simon, 1902

Comments: Endemic to Sri Lanka

XXX.ae. Genus Rhene Thorell, 1869 1. Rhene flavicomans Simon, 1902

Distribution: India, Bhutan, Sri Lanka

Comments: Endemic to South Asia

XXX.af. Genus Saitis Simon, 1876

1. Saitis chaperi Simon, 1885

Distribution: India, Sri Lanka

Comments: Endemic to South Asia

XXX.ag. Genus Sigytes Simon, 1902 1. Sigytes paradisiacus Simon, 1902 Comments: Endemic to Sri Lanka

XXX.ah. Genus Siler Simon, 1889

1. Siler semiglaucus (Simon, 1901)

Synonym: Cyllobelus semiglaucus Simon, 1901

Comments: Endemic to Sri Lanka. Cyllobelus

semiglaucus was transferred to genus Siler by Prószyn'ski (1984)

XXX.ai. Genus Simaetha Thorell, 1881

1. Simaetha cingulata (Karsch, 1891)

Synonym: Homalattus cingulatus Karsch, 1891

Comments: Endemic to Sri Lanka. Homalattus cingulatus was transferred to the genus Simaetha by Simon (1903).

2. Simaetha laminata (Karsch, 1891)

Synonym: Homalattus laminatus Karsch, 1891

Comments: Endemic to Sri Lanka. Homalattus laminatus was transferred to the genus Simaetha by Simon (1903)

3. Simaetha reducta (Karsch, 1891)

Synonym: Homalattus reductus Karsch, 1891

Comments: Endemic to Sri Lanka. Homalattus 2. Ariadna taprobanica Simon, 1906

reductus was transferred to the genus Simaetha by Comments: Endemic to Sri Lanka

Simon (1903).

XXX.aj. Genus Spartaeus Thorell, 1891

1. Spartaeus spinimanus (Thorell, 1878)

Synonym: Boethus spinimanus Thorell, 1878
Spartaeus gracilis Thorell, 1891 Nealces caligatus Simon, 1900 Nealces striatipes Simon, 1900 Boethus striatipes (Simon, 1900) Boethus caligatus (Simon, 1900) Boethus gracilis (Thorell, 1891) Boethuola spinimana (Thorell, 1878) Distribution: Sri Lanka to Borneo Comments: According to Platnick (2006), Boethuola spinimana was unnecessary generic replacement.

XXX.ak. Genus Stagetillus Simon, 1885

1. Stagetillus taprobanicus (Simon, 1902)

Synonym: Padillothorax taprobanicus Simon, 1902

Comments: Endemic to Sri Lanka. Padillothorax taprobanicus was transferred to the genus Stagetillus by Prószyn'ski (1987).

XXX.al. Genus Stergusa Simon, 1889

1. Stergusa aurata Simon, 1902

Comments: Endemic to Sri Lanka

2. Stergusa aurichalcea Simon, 1902

Comments: Endemic to Sri Lanka

3. Stergusa stelligera Simon, 1902

Comments: Endemic to Sri Lanka

XXX.am. Genus Tamigalesus Zabka, 1988

Comments: It is a monotypic genus and endemic to Sri Lanka.

1. Tamigalesus munnaricus Zabka, 1988

Comments: Endemic to Sri Lanka

XXX.an. Genus Telamonia Thorell, 1887

1. Telamonia sponsa (Simon, 1902)

Synonym: Viciria sponsa Simon, 1902

Comments: Endemic to Sri Lanka. Viciria sponsa

was transferred to the genus Telamonia by Prószyn'sk (1984c).

XXX.ao. Genus Thiania C. L. Koch, 1846

1. Thiania pulcherrima C. L. Koch, 1846

Distribution: Sri Lanka, Vietnam, Malaysia, Sulawes

XXX.ap. Genus Uroballus Simon, 1902

1. Uroballus henicurus Simon, 1902

Comments: Endemic to Sri Lanka

2. Uroballus octovittatus Simon, 1902

Comments: Endemic to Sri Lanka

XXX.aq. Genus Viciria Thorell, 1877

1. Viciria polysticta Simon, 1902

Comments: Endemic to Sri Lanka

XXXI. FAMILY SCYTODIDAE BLACKWALL, 1864

XXXI.a. Genus Scytodes Latreille, 1804

1. Scytodes venusta (Thorell, 1890)

Synonym: Dictis venusta Thorell, 1890

Distribution: Sri Lanka to Java, introduced in Netherlands

XXXII. FAMILY SEGESTRIIDAE SIMON, 1893

XXXII.a. Genus Ariadna Audouin, 1826

1. Ariadna oreades Simon, 1906

Comments: Endemic to Sri Lanka

XXXIII. FAMILY SPARASSIDAE BERTKAU, 1872

Comments: Considered senior synonym of Heteropodidae by Jäger (1999).
XXXIII.a. Genus Heteropoda Latreille, 1804

Synonyms: Genus Torania Simon, 1886

Genus Panaretus Simon, 1880

Comments: Genus Torania was synonymised with genus Heteropoda by Jäger (2001) and genus

Panaretus was synonymised with genus

Heteropoda by Jäger (2002).

1. Heteropoda eluta Karsch, 1891

Comments: Endemic to Sri Lanka

2. Heteropoda kandiana Pocock, 1899

Distribution: India, Sri Lanka

Comments: Endemic to South Asia

3. Heteropoda subtilis Karsch, 1891

Comments: Endemic to Sri Lanka

4. Heteropoda umbrata Karsch, 1891

Comments: Endemic to Sri Lanka

XXXIII.b. Genus Olios Walckenaer, 1837

1. Olios ceylonicus (Leardi, 1902)

Synonym: Midamus ceylonicus Leardi, 1902

Comments: Endemic to Sri Lanka

2. Olios greeni (Pocock, 1901)

Synonym: Sparassus greeni Pocock, 1901

Comments: Endemic to Sri Lanka

3. Olios hirtus (Karsch, 1879)

Synonym: Pelmopoda hirta Karsch, 1879

Sparassus hirtus (Karsch, 1879)

Comments: Endemic to Sri Lanka

4. Olios lamarcki (Latreille, 1806)

Synonyms: Thomisus lamarcki Latreille, 1806

Olios captiosus Walckenaer, 1837

Sparassus lamarcki (Latreille, 1806)

Eusparassus lamarcki (Latreille, 1806)

Distribution: Madagascar to Sri Lanka, India

Comments: Sparassus lamarcki was transferred to

genus Olios by Gravely (1931).

4a. Olios lamarcki taprobanicus Strand, 1913 Comments: Endemic to Sri Lanka

5. Olios milleti (Pocock, 1901)

Synonym: Sparassus milleti Pocock, 1901

Distribution: India, Sri Lanka

Comments: Endemic to South Asia. Sparassus milleti

was transferred to genus Olios by Gravely (1931).

6. Olios senilis Simon, 1880

Synonyms: Olios sensilis Simon, 1880

Sparassus senilis (Simon, 1880)

Pelmopoda senilis (Simon, 1880)

Distribution: India, Sri Lanka

Comments: Endemic to South Asia. Original name reinstated by Sethi \& Tikader (1988).

XXXIII.c. Genus Pandercetes L. Koch, 1875

1. Pandercetes decipiens Pocock, 1899

Distribution: India, Sri Lanka

Comments: Endemic to South Asia

2. Pandercetes plumipes (Doleschall, 1859)

Synonym: Delena plumipes Doleschall, 1859

Tychicus plumipes (Doleschall, 1859)

Zatapina plumipes (Doleschall, 1859)

Distribution: Sri Lanka, Amboina, New Guinea

XXXIII.d. Genus Rhitymna Simon, 1897

1. Rhitymna occidentalis Jäger, 2003

Comments: Endemic to Sri Lanka

XXXIII.e. Genus Spariolenus Simon, 1880

1. Spariolenus taprobanicus (Walckenaer, 1837) 
Synonym: Olios taprobanicus Walckenaer, 1837 Comments: Endemic to Sri Lanka. Olios taprobanicus was transferred to the genus Spariolenus by Simon (1880).

XXXIII.f. Genus Stasina Simon, 1877

1. Stasina nalandica Karsch, 1891

Synonym: Stasina nigropiata Pocock, 1899

Thelcticopis nigropicta Pocock, 1900

Comments: Endemic to Sri Lanka. According to

Platnick (2006), Thelcticopis nigropicta was synonymised with Stasina nalandica.

2. Stasina paripes (Karsch, 1879)

Synonym: Themeropis paripes Karsch, 1879

Thelcticopis paripes (Karsch, 1879)

Comments: Endemic to Sri Lanka. According to

Platnick (2006), Thelcticopis paripes was transferred to the genus Stasina.

XXXIII.g. Genus Thelcticopis Karsch, 1884

1. Thelcticopis hercules Pocock, 1901

Comments: Endemic to Sri Lanka

XXXIV. FAMILY STENOCHILIDAE THORELL, 1873

XXXIV.a. Genus Stenochilus O. P.-Cambridge, 1870

1. Stenochilus crocatus Simon, 1884

Synonyms: Metronax crocatus Simon, 1893

Distribution: Myanmar, Cambodia, Sri Lanka

Comments: Original name reinstated by Platnick \& Shadab (1974).

XXXV. FAMILY TETRABLEMMIDAE O.P.CAMBRIDGE, 1873

XXXV.a. Genus Brignoliella Shear, 1978

1. Brignoliella ratnapura Shear, 1988

Comments: Endemic to Sri Lanka

2. Brignoliella scrobiculata (Simon, 1893)

Synonym: Paculla scrobiculata Simon, 1893

Comments: Endemic to Sri Lanka. Paculla

scrobiculata was transferred to the genus Brignoliella by Shear (1978).

XXXV.b. Genus Gunasekara Lehtinen, 1981

Comments: It is monotypic a genus and endemic to

Sri Lanka.

1. Gunasekara ramboda Lehtinen, 1981

Comments: Endemic to Sri Lanka

XXXV.c. Genus Pahanga Shear, 1979

1. Pahanga diyaluma Lehtinen, 1981

Comments: Endemic to Sri Lanka

XXXV.d. Genus Shearella Lehtinen, 1981

1. Shearella lilawati Lehtinen, 1981

Comments: Endemic to Sri Lanka

2. Shearella selvarani Lehtinen, 1981

Comments: Endemic to Sri Lanka

XXXV.e. Genus Tetrablemma O.P.-Cambridge, 1873

1. Tetrablemma medioculatum O.P.-Cambridge, 1873

Comments: Endemic to Sri Lanka

XXXVI. FAMILY TETRAGNATHIDAE MENGE, 1866 Comments: Genera Leucauge and Meta were originally placed in the family Tetragnathidae which is followed by Platnick (2006) and this paper, Tikader (1987) placed it in the family Araneidae without any explanation.

XXXVI.a. Genus Atelidea Simon, 1895

1. Atelidea spinosa Simon, 1895

Comments: Endemic to Sri Lanka
XXXVI.b. Genus Atimiosa Simon, 1895

1. Atimiosa quinquemucronata Simon, 1895

Comments: Endemic to Sri Lanka

XXXVI.c. Genus Dolichognatha O.P.-Cambridge, 1869 1. Dolichognatha nietneri O.P.-Cambridge, 1869 Comments: Endemic to Sri Lanka

XXXVI.d. Genus Leucauge White, 1841

1. Leucauge argentata (O.P.-Cambridge, 1869)

Synonym: Tetragnatha argentata O.P.-Cambridge, 1869

Distribution: India, Sri Lanka, New Guinea

2. Leucauge ditissima (Thorell, 1887)

Synonym: Argyroepeira ditissima Thorell, 1887

Distribution: Sri Lanka, Myanmar

3. Leucauge lamperti Strand, 1907

Comments: Endemic to Sri Lanka

XXXVI.e. Genus Schenkeliella Strand, 1934

Comments: This genus is monotypic and endemic to Sri Lanka.

1. Schenkeliella spinosa (O.P.-Cambridge, 1870)

Synonym: Oeta spinosa O.P.-Cambridge, 1870 Comments: Endemic to Sri Lanka. Strand (1934) provided generic name replacement for the genus Oeta.

XXXVI.f. Genus Tetragnatha Latreille, 1804

Synonym: Genus Eucta Simon, 1881

Comments: Genus Eucta was synonymised with Tetragnatha by Levi (1981).

1. Tetragnatha armata Karsch, 1891

Comments: Endemic to Sri Lanka

2. Tetragnatha determinata Karsch, 1891

Comments: Endemic to Sri Lanka

3. Tetragnatha foveata Karsch, 1891

Distribution: India, Sri Lanka, Maldives

Comments: Endemic to South Asia

4. Tetragnatha geniculata Karsch, 1891

Distribution: Sri Lanka to China

5. Tetragnatha planata Karsch, 1891

Comments: Endemic to Sri Lanka

6. Tetragnatha tenera Thorell, 1881

Distribution: India, Sri Lanka, Queensland

7. Tetragnatha virescens Okuma, 1979

Distribution: Bangladesh, Sri Lanka to Indonesia, Philippines

XXXVI.g. Genus Tylorida Simon, 1894

1. Tylorida culta (O.P.-Cambridge, 1869)

Synonyms: Tetragnatha culta O.P.-Cambridge, 1869

Leucauge sexpustulata Simon, 1906

Leucauge culta (O.P.-Cambridge, 1869)

Anopas cultus (O.P.-Cambridge, 1869)

Distribution: India, Sri Lanka

Comments: Endemic to South Asia. Leucauge culta

was transferred to genus Tylorida by Archer (1951).

XXXVII. FAMILY THERAPHOSIDAE THORELL, 1870

XXXVII.a. Genus Chilobrachys Karsch, 1891

1. Chilobrachys nitelinus Karsch, 1891

Comments: Endemic to Sri Lanka

XXXVII.b. Genus Plesiophrictus Pocock, 1899

Synonym: Genus Heterophrictus Pocock, 1900

Comments: Genus Heterophrictus was synonymised

with genus Plesiophrictus by Raven (1985).
1. Plesiophrictus tenuipes Pocock, 1899

Comments: Endemic to Sri Lanka

XXXVII.c. Genus Poecilotheria Simon, 1885

Comments: Endemic to South Asia.

1. Poecilotheria fasciata (Latreille, 1804)

Synonym: Mygale fasciata Latreille, 1804

Scurria fasciata (Latreille, 1804)

Comments: Endemic to Sri Lanka

2. Poecilotheria ornata Pocock, 1899

Comments: Endemic to Sri Lanka

3. Poecilotheria pederseni Kirk, 2001

Comments: Endemic to Sri Lanka

4. Poecilotheria pococki Charpentier, 1996

Comments: Endemic to Sri Lanka

5. Poecilotheria smithi Kirk, 1996

Comments: Endemic to Sri Lanka

6. Poecilotheria subfusca Pocock, 1895

Synonym: Scurria fasciata Ausserer, 1871

Poecilotheria bara Chamberlin, 1917

Comments: Endemic to Sri Lanka. Poecilotheria bara was synonymised with Poecilotheria subfusca by Kirk (1996).

7. Poecilotheria uniformis Strand, 1913

Comments: Endemic to Sri Lanka

XXXVIII. FAMILY THERIDIIDAE SUNDEVALL, 1833

XXXVIII.a. Genus Argyrodes Simon, 1864

Synonyms: Genus Argyrodina Strand, 1926

Comments: Genera Argyrodina was synonymised with genus Argyrodes by Levi \& Levi (1962)

1. Argyrodes fissifrons O.P.-Cambridge, 1869 Synonyms: Argyrodes inguinalis Thorell, 1878 Argyrodes procastinans O.P.-Cambridge, 1880 Argyrodes scutatus Chrysanthus, 1975

Argyrodes menlunensis Zhu \& Song, 1991

Distribution: India, Sri Lanka to China, Australia

2. Argyrodes flavescens O.P.-Cambridge, 1880 Synonyms: Argyrodes sumatranus Thorell, 1890 Argyrodes miniaceus Bösenberg \& Strand, 1906 Distribution: India, Pakistan, Sri Lanka to Korea, Japan, New Guinea

3. Argyrodes nasutus O.P.-Cambridge, 1880

Synonyms: Argyrodes nasuta O.P.-Cambridge, 1880

Comments: Endemic to Sri Lanka

4. Argyrodes scintillulanus O.P.-Cambridge, 1880 Synonym: Argyrodes scintillulana O.P.-Cambridge, 1880

Distribution: India, Sri Lanka

Comments: Endemic to South Asia

XXXVIII.b. Genus Ariamnes Thorell, 1869

Comments: Genus Ariamnes was removed from the synonym of genus Argyrodes by Agnarsson (2004).

1. Ariamnes pavesii Leardi, 1902

Synonym: Argyrodes pavesii (Leardi, 1902)

Distribution: India, Sri Lanka

Comments: Endemic to South Asia. Original name reinstated by Agnarsson (2004).

XXXVIII.c. Genus Cephalobares O.P.-Cambridge, 1870 Comments: This genus is monotypic and endemic to Sri Lanka. 
1. Cephalobares globiceps O.P.-Cambridge, 1870 Comments: Endemic to Sri Lanka

XXXVIII.d. Genus Chrysso O.P.-Cambridge, 1882 Synonym: Genus Meotipa Simon, 1894 Comments: Genus Meotipa was synonymised with genus Chrysso by Levi \& Levi (1962).

1. Chrysso nigra (O.P.-Cambridge, 1880) Synonym: Argyrodes nigra O.P.-Cambridge, 1880 Theridion oxyurum Thorell, 1890

Theridion nigrum (O. P.-Cambridge, 1880)

Theridula caudata Saito, 1933

Distribution: Sri Lanka to Taiwan, Indonesia

Comments: Theridula caudata was synonymised with Chrysso nigra by Yoshida (1978).

2. Chrysso spiniventris (O.P.-Cambridge, 1869) Synonym: Theridion spiniventre O.P.-Cambridge, 1869 Theridion buitenzorgi Strand, 1907

Chrysso spiniventre (O.P.-Cambridge, 1869)

Distribution: Sri Lanka to Japan (Europe, introduced)

XXXVIII.e. Genus Coscinida Simon, 1895

1. Coscinida gentilis Simon, 1895

Comments: Endemic to Sri Lanka

2. Coscinida novemnotata Simon, 1895

Comments: Endemic to Sri Lanka

3. Coscinida triangulifera Simon, 1904

Distribution: Sri Lanka, Java

Comments: Endemic to Sri Lanka

XXXVIII.f. Genus Dipoena Thorell, 1869

Synonym: Genus Trigonobothrys Simon, 1889

Comments: The genus Trigonobothrys was synonymised with genus Dipoena by Yoshida (2002).

1. Dipoena sertata (Simon, 1895)

Synonym: Stictoxena sertata Simon, 1894

Comments: Endemic to Sri Lanka. According to

Platnick (2006), Stictoxena sertata was nomen nudum.

XXXVIII.g. Genus Emertonella Bryant, 1945 1. Emertonella taczanowskii (Keyserling, 1886)

Synonym: Euryopis taczanowskii Keyserling, 1886 Euryopis floricola Keyserling, 1886

Euryopis nigripes Banks, 1929

Euryopis dentata Gertsch \& Mulaik, 1936

Euryopis rosascostai Mello-Leitão, 1944

Distribution: USA to Argentina, Sri Lanka to

Ryukyu Is.

Comments: Euryopis taczanowskii was transferred to genus Emertonella by Yoshida (2002).

XXXVIII.h. Genus Enoplognatha Pavesi, 1880 Synonym: Genus Symopagia Simon, 1894 Comments: The genus Symopagia was synonymised with the genus Enoplognatha by Levi \& Levi (1962).

1. Enoplognatha oreophila (Simon, 1894)

Synonym: Symopagia oreophila Simon, 1894

Comments: Endemic to Sri Lanka

XXXVIII.i. Genus Episinus Walckenaer, in Latreille, 1809 1. Episinus taprobanicus (Simon, 1895) Synonym: Janulus taprobanicus Simon, 1895 Comments: Endemic to Sri Lanka

XXXVIII.j. Genus Latrodectus Walckenaer, 1805 1. Latrodectus erythromelas Schmidt \& Klaas, 1991 Comments: Endemic to Sri Lanka

XXXVIII.k. Genus Molione Thorell, 1892 1. Molione trispinosa (O.P.-Cambridge, 1873)

Synonym: Phoroncidia trispinosa O. P.-Cambridge, 1873

Comments: Endemic to Sri Lanka. Phoroncidia trispinosa was transferred to the genus Molione by Simon (1894).

XXXVIII.I. Genus Phoroncidia Westwood, 1835 Synonym: Genus Ulesanis L. Koch, 1872

Comments: Genus Ulesanis was synonymised with genus Phoroncidia by Levi \& Levi (1962).

1. Phoroncidia nasuta (O. P.-Cambridge, 1873 Synonym: Stegosoma nasuta O. P.-Cambridge, 1873

Ulesanis nasuta Simon, 1894

Comments: Endemic to Sri Lanka

2. Phoroncidia septemaculeata O. P.-Cambridge, 1873 Comments: Endemic to Sri Lanka

3. Phoroncidia testudo (O.P.-Cambridge, 1873) Synonyms: Ulesanis testudo O.P.-Cambridge, 1873 Stegosoma testudo (O.P.-Cambridge, 1873)

Distribution: India, Sri Lanka

Comments: Endemic to South Asia

4. Phoroncidia thwaitesi O.P.-Cambridge, 1869 Synonym: Phoroncidia thwaitesii O. P.-Cambridge, 1869 Phoroncidia brevispinosa O.P.-Cambridge, 1873 Comments: Endemic to Sri Lanka. Phoroncidia Synonym: Ascuris striatipes Simon, 1897

brevispinosa was synonymised with Phoroncidia Distribution: West Africa, Sri Lanka thwaitesi by Simon (1894).

XXXVIII.m. Genus Propostira Simon, 1894

Comment: Endemic to South Asia.

1. Propostira quadrangulata Simon, 1894 Synonym: Propostira quadrangulosa Simon, 1895 Distribution: India, Sri Lanka

XXXVIII.n. Genus Steatoda Sundevall, 1833 Synonyms: Genus Asagena Sundevall, 1833 Genus Lithyphantes Thorell, 1869

Genus Teutana Simon, 1881

Comments: Genera Asagena, Lithyphantes, Teutana were synonymised with genus Steatoda by Lev (1957).

1. Steatoda rufoannulata (Simon, 1899)

Synonym: Teutana rufoannulata Simon, 1899 Distribution: India, Sri Lanka, Sumatra, Java

XXXVIII.o. Genus Theridion Walckenaer, 1805 1. Theridion albomaculosum O.P.-Cambridge, 1869 Comments: Endemic to Sri Lanka

2. Theridion annulipes O.P.-Cambridge, 1869 Comments: Endemic to Sri Lanka

3. Theridion gabardi Simon, 1895 Comments: Endemic to Sri Lanka

4. Theridion modestum (Simon, 1894) Synonym: Phaetoticus modestus Simon, 1894 Comments: Endemic to Sri Lanka

5. Theridion nodiferum Simon, 1895 Comments: Endemic to Sri Lanka

6. Theridion quadratum (O.P.-Cambridge, 1882) Synonym: Chrysso quadrata O.P.-Cambridge, 1882 Distribution: Sri Lanka, Sumatra

7. Theridion teliferum Simon, 1895 Comments: Endemic to Sri Lanka

XXXVIII.p. Genus Thwaitesia O.P.-Cambridge, 1881 1. Thwaitesia margaritifera O. P.-Cambridge, 1881 Distribution: Sri Lanka, China, Vietnam
XXXIX. FAMILY THERIDIOSOMATIDAE SIMON, 1881

XXXIX.a. Genus Andasta Simon, 1895

1. Andasta semiargentea Simon, 1895

Synonym: Theridiosoma semiargenteum Coddington, 1986

Comments: Endemic to Sri Lanka. Theridiosoma semiargenteum was transferred to the genus Andasta by Saaristo (1996)

XXXIX.b. Genus TheridiosomaO. P.-Cambridge, 1879

1. Theridiosoma genevensium (Brignoli, 1972)

Synonym: Andasta genevensium Brignoli, 1972

Comments: Endemic to Sri Lanka. Andasta genevensium was transferred to the genus Theridiosoma by Saaristo (1996).

\section{FAMILY THOMISIDAE SUNDEVALL, 1833}

XL.a. Genus Ascurisoma Strand, 1928

Synonym: Genus Ascuris

Comments: It is a monotypic genus. The genus Ascuris name was preocuppied and thus, Strand (1928) provided replacement name Ascurisoma for genus Ascuris.

XL.b. Genus Boliscus Thorell, 1891

1. Boliscus decipiens O. P.-Cambridge, 1899

Comments: Endemic to Sri Lanka

XL.c. Genus Borboropactus Simon, 1884

Synonym: Genus Regillus

Comments: The genus Regillus was preoccuppied by Macgillivray (1839) and thus Simon (1884) provided replacement name Borboropactus for the genus Regillus.

1. Borboropactus asper (O.P.-Cambridge, 1884)

Synonym: Regillus asper O.P.-Cambridge, 1884

Regillulus asper Strand, 1942

Comments: Endemic to Sri Lanka

XL.d. Genus Cymbacha L. Koch, 1874

1. Cymbacha simplex Simon, 1895

Comments: Endemic to Sri Lanka

XL.e. Genus Diaea Thorell, 1869

1. Diaea placata O. P.-Cambridge, 1899

Comments: Endemic to Sri Lanka

XL.f. Genus Epidius Thorell, 1877

1. Epidius longipalpis Thorell, 1877

Distribution: India, Sri Lanka, Java, Sumatra,

Ceram, Sulawesi

2. Epidius parvati Benjamin, 2000

Comments: Endemic to Sri Lanka

XL.g. Genus Holopelus Simon, 1886

1. Holopelus piger O. P.-Cambridge, 1899

Comments: Endemic to Sri Lanka

XL.h. Genus Monaeses Thorell, 1869

Synonym: Genus Mecostrabus Simon, 1903

Comments: The genus Mecostrabus was

synonymised with the genus Monaeses by Ono (1985).

1. Monaeses attenuatus O.P.-Cambridge, 1899

Synonym: Rhynchognatha attenuata Crome, 1962

Comments: Endemic to Sri Lanka. Original name reinstated by Platnick (2006).

2. Monaeses cinerascens (Thorell, 1887)

Synonym: Rhynchognatha cinerascens Thorell, 1887 
Distribution: Sri Lanka, Myanmar Comments: Rhynchognatha cinerascens was transferred to the genus Monaeses by Simon (1895).

3. Monaeses greeni O. P.-Cambridge, 1899 Synonym: Rhynchognatha greeni Crome, 1962 Comments: Endemic to Sri Lanka. Original name reinstated by Platnick (2006).

XL.i. Genus Oxytate L. Koch, 1878 Synonym: Genus Dieta Simon, 1880 Comments: Genus Dieta was synonymised with genus Oxytate by Song et al. (1982).

1. Oxytate subvirens (Strand, 1907) Synonym: Dieta subvirens Strand, 1907 Comments: Endemic to Sri Lanka

2. Oxytate taprobane Benjamin, 2001 Comments: Endemic to Sri Lanka

XL.j. Genus Pagida Simon, 1895

1. Pagida salticiformis (O. P.-Cambridge, 1883)

Synonym: Palaephatus salticiformis O. P.-Cambridge, 1883 Comments: Endemic to Sri Lanka. Palaephatus salticiformis was transferred to the genus Pagida by Simon (1895).

XL.k. Genus Peritraeus Simon, 1895 Comments: This genus is monotypic and endemic to Sri Lanka.

1. Peritraeus hystrix Simon, 1895

Comments: Endemic to Sri Lanka.

XL.I. Genus Phrynarachne Thorell, 1869

1. Phrynarachne ceylonica (O.P.-Cambridge, 1884) Synonyms: Ornithoscatoides ceylonica O.P.Cambridge, 1884

Ornithoscatoides nigra O. P.-Cambridge, 1884

Phrynarachne nigra (O. P.-Cambridge, 1884)

Distribution: Sri Lanka to Japan

Comments: Phrynarachne nigra was synonymised with Phrynarachne ceylonica by Ono (1988).

2. Phrynarachne fatalis O. P.-Cambridge, 1899 Comments: Endemic to Sri Lanka

3. Phrynarachne rothschildi Pocock \& Rothschild, 1903 Comments: Endemic to Sri Lanka

XL.m. Genus Runcinia Simon, 1875

1. Runcinia bifrons (Simon, 1895)

Synonym: Runciniopsis bifrons Simon, 1895

Distribution: India, Sri Lanka, Vietnam

XL.n. Genus Stiphropus Gerstäcker, 1873

1. Stiphropus sigillatus (O.P.-Cambridge, 1883)

Synoynm: Casturopoda sigillata O.P.-Cambridge, 1883

Comments: Endemic to Sri Lanka

XL.o. Genus Tagulis Simon, 1895

1. Tagulis mystacinus Simon, 1895

Comments: Endemic to Sri Lanka

XL.p. Genus Talaus Simon, 1886

1. Talaus oblitus O.P.-Cambridge, 1899

Comments: Endemic to Sri Lanka

XL.q. Genus Tarrocanus Simon, 1895

Comments: This genus is endemic to South Asia.

1. Tarrocanus capra Simon, 1895

Comments: Endemic to Sri Lanka

XL.r. Genus Thomisus Walckenaer, 1805

1. Thomisus callidus (Thorell, 1890)

Synonym: Daradius callidus Thorell, 1890
Distribution: Sri Lanka, Singapore, Sumatra, Nias Comments: Endemic to Sri Lanka

S., Java

Comments: According to Platnick (2006), Daradius callidus was transferred to the genus Thomisus.

2. Thomisus granulifrons Simon, 1906

Distribution: India, Sri Lanka

Comments: Endemic to South Asia

\section{FAMILY TITANOECIDAE LEHTINEN, 1967}

XLI.a. Genus Pandava Lehtinen, 1967

1. Pandava laminata (Thorell, 1878)

Synonym: Amaurobius laminatus Thorell, 1878

Amaurobius castaneiceps Simon, 1893

Titanoeca birmanica Thorell, 1895

Amaurobius taprobanicola Strand, 1907

Amaurobius chinesicus Strand, 1907

Titanoeca fulmeki Reimoser, 1927

Syrorisa mumfordi Berland, 1933

Distribution: Sri Lanka to China, New Guinea, Marquesas Is.

Comments: Amaurobius laminatus (Amaurobiidae) was

transferred to genus Pandava by Lehtinen (1967).

\section{FAMILY ULOBORIDAE THORELL, 1869}

XLII.a. Genus Hyptiotes Walckenaer, 1837

1. Hyptiotes analis Simon, 1892

Comments: Endemic to Sri Lanka

XLII.b. Genus Miagrammopes O. P.-Cambridge, 1870 1. Miagrammopes ferdinandi O.P.-Cambridge, 1870 Comments: Endemic to Sri Lanka

2. Miagrammopes thwaitesi O.P.-Cambridge, 1870

Synonym: Miagrammopes thwaitesii O.P.

Cambridge, 1870

Distribution: India, Sri Lanka

Comments: Endemic to South Asia

XLII.c. Genus Uloborus Latreille, 1806

1. Uloborus umboniger Kulczyn'ski, 1908

Comments: Endemic to Sri Lanka

\section{FAMILY ZODARIIDAE THORELL, 1881}

XLIII.a. Genus Hermippus Simon, 1893

Synonym: Genus Hermippoides Gravely, 1921

Comments: The genus Hermippoides was synonymised with genus Hermippus by Jocqué (1986)

1. Hermippus cruciatus Simon, 1905

Distribution: India, Sri Lanka

Comments: Endemic to South Asia

XLIII.b. Genus Suffasia Jocqué, 199

Comments: Endemic to South Asia.

1. Suffasia attidiya Benjamin \& Jocqué, 2000

Comments: Endemic to Sri Lanka

2. Suffasia mahasumana Benjamin \& Jocqué, 2000

Comments: Endemic to Sri Lanka

XLIV. FAMILY ZOROCRATIDAE DAHL, 1913

XLIV.a. Genus Campostichomma Karsch, 1891

Comments: This genus is monotypic and endemic to Sri Lanka

1. Campostichomma manicatum Karsch, 1891

Comments: Endemic to Sri Lanka

XLV. FAMILY ZOROPSIDAE BERTKAU, 1882

XLV.a. Genus Devendra Lehtinen, 1967
1. Devendra pardalis (Simon, 1898)

Synonym: Campostichomma pardale Simon, 1898 Devendra pardale (Simon, 1898)

Comments: Endemic to Sri Lanka. Campostichomma pardale was transferred to the genus Devendra by Lehtinen (1967).

2. Devendra pumilus (Simon, 1898)

Synonym: Campostichomma pumilum Simon, 1898 Devendra pumila Lehtinen, 1967

Comments: Endemic to Sri Lanka. Campostichomma pumila was transferred to the genus Devendra by Lehtinen (1967).

3. Devendra seriatus (Simon, 1898)

Synonym: Campostichomma seriatum Simon, 1898 Devendra seriata Lehtinen, 1967

Comments: Endemic to Sri Lanka. Campostichomma seriatum was transferred to the genus Devendra by Lehtinen (1967).

\section{Sri Lanka spider summary}

Number of Families: 45

Number of Genera: 213

Number of Species: 354

Number of Subspecies: 1

Number of Endemic Families: 0

Number of Endemic Genera: 22

Number of Endemic Species: 246 
genera are endemic to the country and of which 17 are monotypic genera (Table 2). There is only one subspecies reported from this country. There are 246 species endemic to the country (Table 2). Major species have been described by Simon (1880, 1884, 1885, 1886, 1889, 1892, $1893 \mathrm{a}, \mathrm{b}, 1894,1895,1896,1897,1898$, $1900,1901,1902,1903,1904,1905$, $1906,1907)$ by describing 118 species from Sri Lanka.

Species with extralimital distribution

In this paper, we include spider species whose distribution is either Karakorum or Himalaya as per Platnick (2006). The Himalaya and Karakorum are large areas shared by South Asian countries (Pakistan, India, Nepal \& Bhutan) and China. Therefore, endemicity of the spider species reported from these areas cannot be determined until exact location of the species is known. There are four monotypic genera reported from Karakorum and one monotypic genus from the Himalaya. Apart from this, there are 62 species and four subspecies (Table 3 ) reported from Karakorum and Himalaya. Until the exact location of the species is known, we consider these taxa from Karakorum and Himalaya as occurring in South Asia without assigning endemicity.

\section{Changes in the Indian checklist}

Two Indian spider genera Indicoblemma and Bristowia are no more monotypic genera as more species have been described in the last one year. The genus Indicoblemma is no longer endemic to India as new species have been described from non-South Asian countries. Of the 51 species which need taxonomic verification (Siliwal et al., 2005), only one species has been transferred to the correct genus. Amaurobius indicus Bastawade, 2002 was transfered and provided a replacement name Oedignatha raigadensis by the same author (Bastawade, 2006). The number of Indian nomina dubia and incertae sedis species remain the same as given in Siliwal et al. (2005). There are six Indian species that have been recently reported from Bangladesh and two species from China. Platnick (2006) has reported five Indian species (Cyclosa krusa, C. hexatuberculata, C. saismarka, C. spirifera and Clubiona filicata) from Pakistan, but without reference.
Table 3. List of spider species from Himalaya and Karakorum.

Agelenidae

1. Tikaderia psechrina (Simon, 1906)*

Amaurobiidae

2. Coelotes stylifer Caporiacco, 1935

\section{Araneidae}

3. Araneus altitudinum Caporiacco, 1934

4. Araneus obscurissimus Caporiacco, 1934

5. Araneus pontii Caporiacco, 1934

6. Cyclosa kashmirica Caporiacco, 1934

Clubionidae

7. Clubiona crouxi Caporiacco, 1935

Cybaeidae

8. Cedicus bucculentus Simon, 1889*

9. Cybaeus broni Caporiacco, 1934

Gnaphosidae

10. Berlandina drassodea (Caporiacco, 1934)

11. Micaria dives (Lucas, 1846)

11a. Micaria dives concolor (Caporiacco, 1935)

12. Micaria pulcherrima Caporiacco, 1935

12a. Micaria pulcherrima flava Caporiacco, 1935

13. Pterotrichina nova Caporiacco, 1934

14. Sillemia clavifemur Reimoser, 1935

Linyphiidae

15. Alioranus distinctus Caporiacco, 1935

16. Alioranus minutissimus Caporiacco, 1935

17. Araeoncus duriusculus Caporiacco, 1935

18. Bathyphantes glacialis Caporiacco, 1935

19. Bathyphantes larvarum Caporiacco, 1935

20. Bathyphantes reticularis Caporiacco, 1935

1. Erigone pseudovagans Caporiacco, 1935

22. Gongylidiellum chiardolae Caporiacco, 1935

23. Gongylidiellum nigrolimbatum Caporiacco, 1935

24. Gongylidium baltoroi Caporiacco, 1935

25. Lepthyphantes allegrii Caporiacco, 1935

26. Lepthyphantes annulipes Caporiacco, 1935

27. Lepthyphantes deosaicola Caporiacco, 1935

28. Lepthyphantes incertissimus Caporiacco, 1935

29. Lepthyphantes nigridorsus Caporiacco, 1935

30. Lepthyphantes pratorum Caporiacco, 1935

31. Lepthyphantes striatiformis Caporiacco, 1935

32. Lepthyphantes trivittatus Caporiacco, 1935

33. Microctenonyx cavifrons (Caporiacco, 1935)

34. Pocadicnemis desioi Caporiacco, 1935

35. Tiso megalops Caporiacco, 1935

\section{Lycosidae}

36. Acantholycosa baltoroi (Caporiacco, 1935)

37. Evippomma evippiforme (Caporiacco, 1935)

38. Hippasa flavicoma Caporiacco, 1935

39. Hogna rubromandibulata (O. P.-Cambridge, 1885)

40. Pardosa flavisterna Caporiacco, 1935

41. Pardosa hydaspis Caporiacco, 1935

42. Pardosa vindicata (O. P.-Cambridge, 1885)

43. Schizocosa concolor (Caporiacco, 1935)

Nemesiidae

44. Raveniola concolor Zonstein, 2000*

Philodromidae

45. Thanatus balestrerii Caporiacco, 1935

46. Vacchellia baltoroi Caporiacco, 1935

Salticidae

47. Ballognatha typica Caporiacco, 1935

48. Chalcoscirtus flavipes Caporiacco, 1935

49. Dendryphantes caporiaccoi Roewer, 1951
50. Dolichoneon typicus Caporiacco, 1935

51. Euophrys concolorata Roewer, 1951

52. Pseudicius pseudoicioides (Caporiacco, 1935)*

Sparassidae

53. Eusparassus flavovittatus Caporiacco, 1935

54. Eusparassus pontii Caporiacco, 1935

Theridiidae

55. Euryopis megalops (Caporiacco, 1934)

56. Euryopis venutissima (Caporiacco, 1934)

57. Theridion angustifrons Caporiacco, 1934

58. Theridion glaciale Caporiacco, 1934

59. Theridion latisternum Caporiacco, 1934

60. Theridion sisyphium (Clerck, 1757)

60a. Theridion sisyphium torandae Strand, 1917

Thomisidae

61. Ebrechtella tricuspidata (Fabricius, 1775)

61a. Ebrechtella tricuspidata concolor (Caporiacco, 1935)

62. Ozyptila spinosissima Caporiacco, 1934

Note: Himalayas species are marked with '*' and rest of the species are from Karakorum.

Species with original name reinstated

Original names have been reinstated for 36 species of South Asian spiders, mainly lycosids (16 species) that were transferred to other genera without detailed explanations for their transfer and these changes were not accepted by many authors including Platnick (2006). Of these, 25 species are from India (Siliwal et al., 2005), eight from Sri Lanka, one each from Afghanistan, Karakorum and Pakistan and nine species which occur in more than one South Asian country. Authors that reinstated original names (apart from Indian species as given in Siliwal et al., 2005) are Agnarsson (2004), Brignoli (1976, 1983, 1986), Logunov (2001), Platnick (2006), Platnick \& Shadab (1974), Tikader \& Malhotra (1980), and Helsdingen (1979).

\section{Species with new combination}

There are only two species (from India and Bhutan) with new combination, Dictyna rebai Tikader, 1966 (Dictynidae) and Coelotes baronii Brignoli, 1978 (Amaurobiidae). The male and female of Coelotes baronii identified by Brignoli (1978) was transferred by Wang (2002) to the genus Draconarius (male) and to the genus Himalcoelotes (female). The transfer of Dictyna rebai was discussed in Siliwal et al. (2005).

Taxa not included in the World Catalog of Spiders

There are 39 species of spiders 
described from India that have not yet identified, these spider species published found a place in the World Catalog of in refereed Indian journals are to be Spiders (Platnick, 2006). Unless recognized as valid species. They are otherwise proved to be wrongly listed in Table 4.

\section{Table 4. List of Indian species that have not been included by Platnick (2006).}

\section{Agelenidae}

1. Tegenaria comstocki Gajbe, 2004

Araneidae

2. Larinia bharatae Bhandari \& Gajbe, 2001

3. Neoscona biswasi Bhandari \& Gajbe, 2001

4. Neoscona dyali Gajbe, 2004

5. Neoscona parambikulamensis Patel, 2003

6. Neoscona sanghi Gajbe, 2004

Corinnidae

7. Oedignatha shillongensis Biswas \& Majumder, 1995

Gnaphosidae

8. Sosticus jabalpurensis Bhandari \& Gajbe, 2001

Lycosidae

9. Lycosa shaktae Bhandari \& Gajbe, 2001

10. Pardosa ranjani Gajbe, 2004

Oonopidae

11. Triaeris barela Gajbe, 2004

Oxyopidae

12. Oxyopes tikaderi Biswas \& Majumder, 1995

Philodromidae

13. Philodromus sanjeevi Gajbe, 2004

14. Thanatus ketani Bhandari \& Gajbe, 2001

Salticidae

15. Marpissa manipuriensis Biswas \& Biswas, 2004

16. Myrmarachne megachelae Ganesh Kumar \&

Mahanasundaram, 1998

17. Phidippus bhimrakshiti Gajbe, 2004

18. Rhene haldanei Gajbe, 2004

19. Rhene sanghrakshiti Gajbe, 2004

Scytodidae

20. Scytodes alfredi Gajbe, 2004

Tetragnathidae

21. Pachygantha silentvalliensis Biswas and Roy, 2004

22. Tetragnatha chamberlini (Gajbe, 2004)

Thomisidae

23. Misumenoides gwarighatensis Gajbe, 2004

24. Ozyptila jabalpurensis Bhandari \& Gajbe, 2001

25. Runcinia khandari Gajbe, 2004

26. Runcinia sitadongri Gajbe, 2004

27. Strigoplus moluri Patel, 2003

28. Thomisus baghdeoi Gajbe, 2004

29. Thomisus bargi Gajbe, 2004

30. Thomisus danieli Gajbe, 2004

31. Thomisus kokiwadai Gajbe, 2004

32. Thomisus pateli Gajbe, 2004

33. Thomisus pathaki Gajbe, 2004

34. Thomisus rajani Bhandari \& Gajbe, 2001

35. Thomisus simoni Gajbe, 2004

36. Thomisus viveki Gajbe, 2004

37. Thomisus whitakeri Gajbe, 2004

38. Xysticus tikaderi Bhandari \& Gajbe, 2001

Uloboridae

39. Uloborus jabalpurensis Bhandari \& Gajbe, 2001

\section{Species with misspelt generic name}

There are six genera of South Asian spiders, which were misspelt in the original text or by various arachnologists. These genera are Chorizopes Cambridge, 1870, Megamyrmaekion Wider, 1834, Castianeira Keyserling, 1879, Scopoides Platnick, 1989, Troxochrota Kulczyn'ski, 1894 and Zelotes Gistel, 1848. Notes for such changes are given in the main checklist under comments for the respective species.

\section{Species identified as nomen dubium}

There are 29 species from South Asia, whose type specimen is not in proper condition or is incomplete and are categorised as nomen dubium. The original name of such species is retained until further specimens are available. Of these 29 species, 21 are from India, six from Sri Lanka, one each from Afghanistan and Karakorum. South Asian species categorised as nomen dubium other than India are listed in Table 5.

\section{Species identified as incertae sedis \\ Species with uncertain taxonomic}

Table 5. List of nomen dubium species in South Asia (except India).

\section{Family Oonopidae}

1. Camptoscaphiella fulva Caporiacco, 1934

(Karakorum)

Reference: Brignoli (1976)

Family Salticidae

1. Heliophanus vittatus Denis, 1958 (Afghanistan)

Reference: Roewer (1955)

2. Euophrys declivis Karsch, 1879 (Sri Lanka)

Reference: Roewer (1955)

3. Hasarius arcigerus Karsch, 1891 (Sri Lanka)

Reference: Roewer (1955)

4. Icius discatus Karsch, 1891 (Sri Lanka)

Reference: Roewer (1955)

5. Maevia roseolimbata Hasselt, 1893 (Sri Lanka) Reference: Roewer (1955)

6. Rhene tamula Karsch, 1879 (Sri Lanka)

Reference: Roewer (1955)

7. Salticus pravus Karsch, 1880 (Sri Lanka)

Reference: Roewer (1955) position are categorised as incertae sedis. Five species from South Asia are identified as incertae sedis, three from India (Siliwal et al., 2005), one each from Pakistan and Sri Lanka.

The Pakistan species Altella conglobata Dyal, 1935 (Uloboridae) was identified as incertae sedis by Lehtinen (1967) and the Sri Lankan species Taphiassa punctigera Simon, 1895 (Mysmenidae/ Theridiidae) was identified as incertae sedis by Brignoli (1980) as both the species did not possess characters of the respective genera.

\section{Species identified as nomen nudum}

Sivaperuman et al. (2005) listed Rhomphaea vansdaensis in the checklist of spiders from Parambikulam Wildlife Sanctuary. This species lacks formal description and thus we assign it as nomen nudum.

Species/generic names with emendation In addition to 37 species from India (Siliwal et al., 2005), there is a single species Araneus formosellus (Roewer, 1942) from Pakistan whose name was emended as per ICZN rules by Platnick (2006).

Taxa that have undergone changes in their taxonomic position

There are 260 taxa of South Asia that have been transferred to other families or genera by various authors (see Siliwal et al., 2005, \& Platnick, 2006). Apart from this there have been 142 taxa that were synonymised with other spider families/ genera/species in South Asia. List of South Asian taxa excluding India that have undergone changes in taxonomic position are listed in Tables 6 and 7 . Amongst the recent changes in taxonomic positions, the subfamily Nephilinae (Tetragnathidae) was elevated to the family level by Kuntner (2006) and hence the four genera Clitaetra Simon, 1889, Herennia Thorell, 1877, Nephila Leach, 1815 and Nephilengys L. Koch, 1872 were transferred from Tetragnathidae to the family Nephilidae. Since the publication of Siliwal et al. (2005) there have been three Indian species transfered to another family or genus amongst. Spariolenus minusculus (Reimoser 1934) of the family Sparassidae was transferred to the genus Martensopoda by Jäger (2006); Araneus himalayaensis of the family Araneidae was 
Table 6. List of South Asian spider genera/species that have undergone transfer in their taxonomic position

\begin{tabular}{lll}
\hline Valid species name & Transferred from & Transferred to \\
\hline Transfer of genus to different family & & Catalogue \# \\
Genus Heligmomerus & Ctenizidae & Idiopidae \\
Genus Scalidognathus & Ctenizidae & Idiopidae \\
Genus Sphingius & Clubionidae & Liocranidae \\
Genus Cheiracanthium & Clubionidae & Miturgidae \\
Genus Anagraphis & Prodidomidae & Gnaphosidae \\
Genus Clitaetra & Tetragnathidae & Nephilidae \\
Genus Herennia & Tetragnathidae & Nephilidae \\
Genus Nephila & Tetragnathidae & Nephilidae \\
Genus Nephilengys & Tetragnathidae & Nephilidae \\
Genus Philodromus & Thomisidae & Philodromidae \\
Genus Thanatus & Thomisidae & Philodromidae \\
Genus Tibellus & Thomisidae & PXIIIl.a. \\
& XXXVI.a. & XXXVI.b. \\
XXXVI.c. & XXXVI.d. \\
XLIII.d. \\
XLIII.f.
\end{tabular}

Transfer of species to different genus/family

Tikaderia psechrina (Simon, 1906)

Genus Malthonica (Agelenidae)

Genus Tikaderia (Agelenidae)

I.c. 1

Draconarius baronii (Brignoli, 1978)

Genus Coelotes (Amaurobiidae)

Draconarius gurkha (Brignoli, 1976)

Draconarius schenkeli (Brignoli, 1978)

Draconarius stemmleri (Brignoli, 1978)

Draconarius wuermlii (Brignoli, 1978)

Himalcoelotes brignolii Wang, 2002

Himalcoelotes gyirongensis ( $\mathrm{Hu} \& \mathrm{Li}, 1987$ )

Himalcoelotes sherpa (Brignoli, 1976)

Metanapis montisemodi (Brignoli, 1978)

Metanapis tectimundi (Brignoli, 1978)

Araneus ellipticus (Tikader \& Bal, 1981)

Araneus nympha (Simon, 1889)

Eriovixia excelsa (Simon, 1889)

Hypsosinga taprobanica (Simon, 1895)

Neoscona pavida (Simon, 1906)

Nusatidia bimaculata (Simon, 1897)

Koppe armata (Simon, 1896)

Utivarachna accentuata (Simon, 1896)

Anemesia tubifex (Pocock, 1889)

Anaxibia nigricauda (Simon, 1905)

Dictynomorpha smaragdula (Simon, 1905)

Asiabadus asiaticus (Charitonov, 1946)

Alistra radleyi (Simon, 1898)

Alistra stenura (Simon, 1898)

Alistra taprobanica (Simon, 1898)

Neotama variata (Pocock, 1899)

Ceratinopsis monticola (Simon, 1894)

Gorbothorax wunderlichi (Brignoli, 1983)

Himalaphantes grandiculus (Tanasevitch, 1987)

Himalaphantes magnus (Tanasevitch, 1987)

Himalaphantes martensi (Thaler, 1987)

Indophantes digitulus (Thaler, 1987)

Labullinyphia tersa (Simon, 1894)

Microbathyphantes palmarius (Marples, 1955)

Nesioneta benoiti (van Helsdingen, 1978)

Tenuiphantes plumipes (Tanasevitch, 1987)

Argistes seriatus (Karsch, 1891)

Anomalosa harishi (Dyal, 1935)

Arctosa bakva (Roewer, 1960)

Arctosa mulani (Dyal, 1935)

Arctosa raptor (Kulczyn'ski, 1885)

Hogna himalayensis (Gravely, 1924)

Genus Coelotes (Amaurobiidae)

Genus Coelotes (Amaurobiidae)

Genus Coelotes (Amaurobiidae)

Genus Paracoelotes (Amaurobiidae)

Genus Coelotes (Amaurobiidae)

Genus Coelotes (Amaurobiidae)

Genus Coelotes (Amaurobiidae)

Genus Pseudanapis (Anapidae)

Genus Pseudanapis (Anapidae)

Genus Neoscona (Araneidae)

Genus Epeira (Araneidae)

Genus Neoscona (Araneidae)

Genus Pronous (Araneidae)

Genus Araneus (Araneidae)

Genus Matidia (Clubionidae)

Genus Medmassa (Corinnidae)

Genus Trachelas (Corinnidae)

Genus Nemesia (Nemesiidae)

Genus Dictyna (Dictynidae)

Genus Dictyna (Dictynidae)

Genus Scotophaeus (Gnaphosidae)

Genus Aviola (Hahniidae)

Genus Aviola (Hahniidae)

Genus Hahnia (Hahniidae)

Genus Tama (Hersilidae)

Genus Lygarina (Linyphiidae)

Genus Oedothorax (Linyphiidae)

Genus Lepthyphantes (Linyphiidae)

Genus Lepthyphantes (Linyphiidae)

Genus Lepthyphantes (Linyphiidae)

Genus Lepthyphantes (Linyphiidae)

Genus Linyphia (Linyphiidae)

Genus Priscipalpus (Linyphiidae)

Genus Lepthyphantes (Linyphiidae)

Genus Meioneta (Linyphiidae)

Genus Lepthyphantes (Linyphiidae)

Genus Leptodrassus (Liocranidae)

Genus Anomalomma (Lycosidae)

Genus Arkalosula (Lycosidae)

Genus Pardosa (Lycosidae)

Genus Trochosa (Lycosidae)

Genus Lycosa (Lycosidae)

Genus Sschizocosa (Lycosidae)

Hogna lupina (Karsch, 1879)

Ocyale lanca (Karsch, 1879)

Pardosa basiri (Dyal, 1935)

Pardosa shyamae (Tikader, 1970)

Pardosa timidula (Roewer, 1951)

Wadicosa quadrifera (Gravely, 1924)

Nesticella aelleni (Brignoli, 1972)

Genus Lycosa (Lycosidae)

Genus Lycosa (Lycosidae)

Genus Lycosa (Lycosidae)

Genus Allocosa (Lycosidae)

Genus Lycosa (Lycosidae)

Genus Nesticus (Nesticidae)

Genus Nesticus (Nesticidae)

Genus Althepus (Ochyroceratidae)

Nesticella nepalensis (Hubert, 1973)

Genus Althepus (Ochyroceratidae)

Psiloderces mulcatus (Brignoli, 1973)

Genus Merizocera (Ochyroceratidae)

Psiloderces elasticus (Brignoli, 1975)

Genus Psilochorus (Pholcidae)

Perenethis dentifasciata (O. P.-Cambridge, 1885)

Genus Pisaura (Pisauridae)

Genus Draconarius (Amaurobiidae)

Genus Draconarius (Amaurobiidae)

Genus Draconarius (Amaurobiidae)

Genus Draconarius (Amaurobiidae)

Genus Draconarius (Amaurobiidae)

Genus Himalcoelotes (Amaurobiidae)

Genus Himalcoelotes (Amaurobiidae)

Genus Himalcoelotes (Amaurobiidae)

Genus Metanapis (Anapidae)

Genus Metanapis (Anapidae)

Genus Araneus (Araneidae)

Genus Araneus (Araneidae)

Genus Eriovixia (Araneidae)

Genus Hypsosinga (Araneidae)

Genus Neoscona (Araneidae)

Genus Nusatidia (Clubionidae)

Genus Koppe (Corinnidae)

Genus Utivarachna (Corinnidae)

Genus Anemesia (Cyrtaucheniidae)

Genus Anaxibia (Dictynidae)

Genus Dictynomorpha (Dictynidae)

Genus Asiabadus (Gnaphosidae)

Genus Alistra (Hahniidae)

Genus Alistra (Hahniidae)

Genus Alistra (Hahniidae)

Genus Neotama (Hersilidae)

Genus Ceratinopsis (Linyphiidae)

Genus Gorbothorax (Linyphiidae)

Genus Himalaphantes (Linyphiidae)

Genus Himalaphantes (Linyphiidae)

Genus Himalaphantes (Linyphiidae)

Genus Indophantes (Linyphiidae)

Genus Labullinyphia (Linyphiidae)

Genus Microbathyphantes (Linyphiidae)

Genus Mughiphantes (Linyphiidae)

Genus Nesioneta (Linyphiidae)

Genus Tenuiphantes (Linyphiidae)

Genus Argistes (Liocranidae)

Genus Anomalosa (Lycosidae)

Genus Arctosa (Lycosidae)

Genus Arctosa (Lycosidae)

Genus Arctosa (Lycosidae)

Genus Hogna (Lycosidae)

Genus Hogna (Lycosidae)

Genus Ocyale (Lycosidae)

Genus Pardosa (Lycosidae)

Genus Pardosa (Lycosidae)

Genus Pardosa (Lycosidae)

Genus Wadicosa (Lycosidae)

Genus Nesticella (Nesticidae)

Genus Nesticella (Nesticidae)

Genus Leclercera (Ochyroceratidae)

Genus Psiloderces (Ochyroceratidae)

Genus Psiloderces (Ochyroceratidae)

Genus Holocneminus (Pholcidae)

Genus Perenethis (Pisauridae)
II.c. 1

II.c. 2

II.c. 3

II.c. 4

II.c. 5

II.d.2

Il.d.5

II.d.8

III.a.1

III.a.2

V.c. 8

V.c. 19

V.I.1

V.r.1

V.x.15

IX.c.1

X.i.1

X.n.1

XV.a.1

XVIII.b.1

XVIII.f.3

XXIII.c. 1

XXIV.a.1

XXIV.a.2

XXIV.a.3

XXV.d.3

XXIX.j.1

XXIX.q.5

XXIX.u.1

XXIX.u.2

XXIX.u.3

XXIX.w.2

XXIX.y.1

XXIX.ae.1

XXIX.ag.1

XXIX.aj.1

XXIX.as.1

XXX.a.1

XXXI.e.1

XXXI.f.1

XXXI.f.9

XXXI.f.10

XXXI.m.2

XXXI.m.3

XXXI.q.3

XXXI.r.8

XXXI.r.53

XXXI.r.62

XXXI.X.1

XXXVII.b.1

XXXVII.b.2

XXXVIII.b.1

XXXVIII.d.1

XXXVIII.d.2

XLIV.d.1

XLVI.g.1 


\begin{tabular}{|c|c|c|c|}
\hline Valid species name & Transferred from & Transferred to & Catalogue \# \\
\hline Cosmophasis olorina (Simon, 1901) & Genus Telamonia (Salticidae) & Genus Cosmophasis (Salticidae) & XLIX.p.1 \\
\hline Cotinusa splendida (Dyal, 1935) & Genus Gophoa (Salticidae) & Genus Cotinusa (Salticidae) & XLIX.q.1 \\
\hline Evarcha cancellata (Simon, 1902) & Genus Colopsus (Salticidae) & Genus Evarcha (Salticidae) & XLIX.ac.1 \\
\hline Hyllus indicus (Tikader, 1974) & Genus Phidippus (Salticidae) & Genus Hyllus (Salticidae) & XLIX.ar.3 \\
\hline Pseudicius pseudoicioides (Caporiacco, 1935) & Genus Icius (Salticidae) & Genus Pseudicius (Salticidae) & XLIX.ca.11 \\
\hline Langona aperta (Denis, 1958) & Genus Aelurillus (Salticidae) & Genus Langona (Salticidae) & XLIX.ax.1 \\
\hline Marengo inornata (Simon, 1900) & Genus Philates (Salticidae) & Genus Marengo (Salticidae) & XLIX.az.2 \\
\hline Marpissa fornicis (Dyal, 1935) & Genus Hyctia (Salticidae) & Genus Marpissa (Salticidae) & XLIX.ba.7 \\
\hline Neobrettus tibialis (Prószyn'ski, 1978) & Genus Cyrba (Salticidae) & Genus Neobrettus (Salticidae) & XLIX.bf.1 \\
\hline Phintella multimaculata (Simon, 1901) & Genus Chrysilla (Salticidae) & Genus Phintella (Salticidae) & XLIX.br.8 \\
\hline Phintella suavis (Simon, 1885) & Genus Telamonia (Salticidae) & Genus Phintella (Salticidae) & XLIX.br.12 \\
\hline Phintella volupe (Karsch, 1879) & Genus Attus (Salticidae) & Genus Phintella (Salticidae) & XLIX.br.15 \\
\hline Phlegra dhakuriensis (Tikader, 1974) & Genus Marpissa (Salticidae) & Genus Phlegra (Salticidae) & XLIX.bs.1 \\
\hline Phlegra particeps (O. P.-Cambridge, 1872) & Genus Salticus (Salticidae) & Genus Phlegra (Salticidae) & XLIX.bs.2 \\
\hline Portia labiata (Thorell, 1887) & Genus Erasinus (Salticidae) & Genus Portia (Salticidae) & XLIX.by.4 \\
\hline \multicolumn{4}{|l|}{ Pseudicius afghanicus (Andreeva, Heciak \& } \\
\hline Prószyn'ski, 1984) & Genus Icius (Salticidae) & Genus Pseudicius (Salticidae) & XLIX.ca.1 \\
\hline Pseudicius flavipes (Caporiacco, 1935) & Genus Icius (Salticidae) & Genus Pseudicius (Salticidae) & XLIX.ca.6 \\
\hline Rafalus wittmeri (Prószyn'ski, 1978) & Genus Aelurillus (Salticidae) & Genus Rafalus (Salticidae) & XLIX.cd.1 \\
\hline Siler semiglaucus (Simon, 1901) & Genus Cyllobelus (Salticidae) & Genus Siler (Salticidae) & XLIX.ci.1 \\
\hline Simaetha cingulata (Karsch, 1891) & Genus Homalattus (Salticidae) & Genus Simaetha (Salticidae) & XLIX.cj.1 \\
\hline Simaetha laminata (Karsch, 1891) & Genus Homalattus (Salticidae) & Genus Simaetha (Salticidae) & XLIX.cj.2 \\
\hline Simaetha reducta (Karsch, 1891) & Genus Homalattus (Salticidae) & Genus Simaetha (Salticidae) & XLIX.cj.3 \\
\hline Sitticus niveosignatus (Simon, 1880) & Genus Attus (Salticidae) & Genus Sitticus (Salticidae) & XLIX.cl.3 \\
\hline Stagetillus taprobanicus (Simon, 1902) & Genus Padillothorax (Salticidae) & Genus Stagetillus (Salticidae) & XLIX.cn.1 \\
\hline Telamonia dimidiata (Simon, 1899) & Genus Viciria (Salticidae) & Genus Telamonia (Salticidae) & XLIX.cs.1 \\
\hline Telamonia sponsa (Simon, 1902) & Genus Viciria (Salticidae) & Genus Telamonia (Salticidae) & XLIX.cs.7 \\
\hline Olios iranii (Pocock, 1901) & Genus Sparassus (Sparassidae) & Genus Olios (Sparassidae) & LIV.f.10 \\
\hline Olios lamarcki (Latreille, 1806) & Genus Sparassus (Sparassidae) & Genus Olios (Sparassidae) & LIV.f.12 \\
\hline Olios lutescens (Thorell, 1894) & Genus Sparassus (Sparassidae) & Genus Olios (Sparassidae) & LIV.f.13 \\
\hline Olios milleti (Pocock, 1901) & Genus Sparassus (Sparassidae) & Genus Olios (Sparassidae) & LIV.f.14 \\
\hline Olios tener (Thorell, 1891) & Genus Sparassus (Sparassidae) & Genus Olios (Sparassidae) & LIV.f.28 \\
\hline Pseudopoda prompta (O.P.-Cambridge, 1885) & Genus Heteropoda (Sparassidae) & Genus Pseudopoda (Sparassidae) & LIV.i.33 \\
\hline Spariolenus taprobanicus (Walckenaer, 1837) & Genus Olios (Sparassidae) & Genus Spariolenus (Sparassidae) & LIV.I.3 \\
\hline Stasina paripes (Karsch, 1879) & Genus Thelcticopis (Sparassidae) & Genus Stasina (Sparassidae) & LIV.m.2 \\
\hline Brignoliella martensi (Brignoli, 1972) & Genus Paculla (Tetrablemmidae) & Genus Brignoliella (Tetrablemmidae) & LVI.a.2 \\
\hline Brignoliella scrobiculata (Simon, 1893) & Genus Paculla (Tetrablemmidae) & Genus Brignoliella (Tetrablemmidae) & LVI.a.4 \\
\hline Tylorida culta (O. P.-Cambridge, 1869) & Genus Leucauge (Tetragnathidae) & Genus Tylorida (Tetragnathidae) & LVII.n.1 \\
\hline Molione trispinosa (O. P.-Cambridge, 1873) & Genus Phoroncidia (Theridiidae) & Genus Molione (Theridiidae) & LIX.o.1 \\
\hline Andasta semiargentea Simon, 1895 & Genus Theridiosoma (Theridiosomatidae) & Genus Andasta (Theridiosomatidae) & LX.a.1 \\
\hline Theridiosoma genevensium (Brignoli, 1972) & Genus Andasta (Theridiosomatidae) & Genus Theridiosoma (Theridiosomatidae) & LX.b.1 \\
\hline Ebrechtella concinna (Thorell, 1877) & Genus Diaea (Thomisidae) & Genus Ebrechtella (Thomisidae) & LXI.k.1 \\
\hline Ebrechtella pseudovatia (Schenkel, 1936) & Genus Misumenops (Thomisidae) & Genus Ebrechtella (Thomisidae) & LXI.k.2 \\
\hline Ebrechtella sufflava (O. P.-Cambridge, 1885) & Genus Misumenops (Thomisidae) & Genus Ebrechtella (Thomisidae) & LXI.k.3 \\
\hline Lysiteles excultus (O. P.-Cambridge, 1885) & Genus Synema (Thomisidae) & Genus Lysiteles (Thomisidae) & LXI.r.6 \\
\hline Monaeses cinerascens (Thorell, 1887) & Genus Rhynchognatha (Thomisidae) & Genus Monaeses (Thomisidae) & LXI.x.3 \\
\hline Pagida salticiformis (O. P.-Cambridge, 1883) & Genus Palaephatus (Thomisidae) & Genus Pagida (Thomisidae) & LXI.aa.1 \\
\hline Runcinia spinulosa (O. P.-Cambridge, 1885) & Genus Diaea (Thomisidae) & Genus Runcinia (Thomisidae) & LXI.ai.10 \\
\hline Thomisus callidus (Thorell, 1890) & Genus Daradius (Thomisidae) & Genus Thomisus (Thomisidae) & LXI.aq.8 \\
\hline Pandava laminata (Thorell, 1878) & Genus Amaurobius (Amaurobiidae) & Genus Pandava (Titanoecidae) & LXII.b.1 \\
\hline Devendra pardalis (Simon, 1898) & Genus Campostichomma (Zoropsidae) & Genus Devendra (Zoropsidae) & LXVII.a.1 \\
\hline Devendra pumilus (Simon, 1898) & Genus Campostichomma (Zoropsidae) & Genus Devendra (Zoropsidae) & LXVII.a.2 \\
\hline Devendra seriatus (Simon, 1898) & Genus Campostichomma (Zoropsidae) & Genus Devendra (Zoropsidae) & LXVII.a.3 \\
\hline
\end{tabular}

Note: Catalogue \# in this table refers to the number given in the South Asian checklist (Checklist 9 in web supplement).

transferred to the genus Eriophora by Zhang, Zhu \& Song (2006); and Amaurobius indicus of the family Amaurobiidae was transferred to the genus Oedignatha under the family Corrinidae by Bastawade (2006). However, since Oedignatha indica was preoccupied by Reddy \& Patel, 1993, Bastawade (2006) provided a replacement name Oedignatha raigadensis for this species. Selenocosmia himalayana of the family Theraphosidae was transferred to the genus Haplocosmia by Schmidt (2004). The South Asian species Herennia ornatissima was synonymised with Herennia multipuncta by Kuntner (2005).

\section{REFERENCES}

Agnarsson, I. (2004). Morphological phylogeny of cobweb spiders and their relatives (Araneae: Araneoidea: Theridiidae). Zoological Journal of the Linnean Society 141: 447-626.
Alderweireldt, M. \& R. Jocqué (2005). A taxonomic review of the Afrotropical representatives of the genus Hippasa (Araneae: Lycosidae). Journal of Afrotropical Zoology 2: 45-68.

Andreeva, E.M., S. Heciak \& J. Prószyn'ski (1984). Remarks on Icius and Pseudicius (Araneae: Salticidae) mainly from central Asia. Annales zoologici. Warszawa 37: 349-375.

Archer, A.F. (1951). Studies in the orbweaving spiders (Argiopidae). 1. American Museum Novitates 1487: 1-52.

Baehr, M. \& B. Baehr (1993). The Hersiliidae of the Oriental Region including New Guinea. Taxonomy, phylogeny, zoogeography (Arachnida: Araneae). Spixiana (Suppl.) 19: 1-96.

Banks, N. (1894). Notes on Larinia and Cercidia. Entomology Neres 5: 8-9. Banks, N. (1898). Arachnida from Baja California and other parts of Mexico. Proceedings of California Academy of Science 3(1): 205-308.

Barrion, A.T. \& J.A. Litsinger (1995). Riceland Spiders of South and Southeast Asia. CAB International, Wallingford, UK, xix+700pp.

Bastawade, D.B. (2006). Replacement name for Amaurobius indicus 
Table 7. South Asian spider taxa synonymised with other families/genera/species

\begin{tabular}{|c|c|c|}
\hline Old name & Valid Name & Catalogue \# \\
\hline Family Heteropodidae & Family Sparassidae & LIV \\
\hline \multicolumn{3}{|l|}{ Family Amaurobiidae } \\
\hline Coelotes lama Brignoli, 1976 & Draconarius gurkha (Brignoli, 1976) & II.c.2 \\
\hline \multicolumn{3}{|l|}{ Family Araneidae } \\
\hline Argiope shillongensis Sinha, 1951 & Argiope minuta Karsch, 1879 & V.e.7 \\
\hline Cyclosa fissicauda Simon, 1889 & Cyclosa quinqueguttata (Thorell, 1881) & V.h.19 \\
\hline Cyclosa hybophora (Thorell, 1887) & Cyclosa quinqueguttata (Thorell, 1881) & V.h.19 \\
\hline Gea corbetti Tikader, 1982 & Gea subarmata Thorell, 1890 & V.n.2 \\
\hline \multicolumn{3}{|l|}{ Family Barychelidae } \\
\hline Sason armatoris Pocock, 1900 & Sason robustum (O.P.-Cambridge, 1883) & VII.c.2 \\
\hline Sason cinctipes (Pocock, 1892) & Sason robustum (O.P.-Cambridge, 1883) & VII.c.2 \\
\hline \multicolumn{3}{|l|}{ Family Clubionidae/Corrinidae/Mitturgidae } \\
\hline Clubiona atwali Singh, 1970 & Clubiona drassodes O.P.-Cambridge, 1874 & IX.a.10 \\
\hline \multicolumn{3}{|l|}{ Family Ctenidae } \\
\hline Ctenus cuspidatus F. O. P.-Cambridge, 1902 & Ctenus ceylonensis F. O. P.-Cambridge, 1897 & XII.b.3 \\
\hline \multicolumn{3}{|l|}{ Family Ctenizidae/Idiopidae } \\
\hline Genus Nemesiellus & Genus Scalidognathus & XXVIII.c. \\
\hline \multicolumn{3}{|l|}{ Family Gnaphosidae } \\
\hline Drassodes mazurae Esyunin \& Tuneva, 2002 & Drassodes lutescens (C. L. Koch, 1839) & XXIII.g.18 \\
\hline Liodrassus mandae Tikader \& Gajbe, 1977 & Setaphis browni (Tucker, 1923) & XXIII.ac.1 \\
\hline Prosthesima iusta Kulczyn'ski, 1911 & Zelotes sarawakensis (Thorell, 1890) & XXIII.ak.30 \\
\hline \multicolumn{3}{|l|}{ Family Hersiliidae } \\
\hline Hersilia clathrata Thorell, 1895 & Hersilia savignyi Lucas, 1836 & XXV.a.3 \\
\hline \multicolumn{3}{|l|}{ Family Linyphiidae } \\
\hline Microbathyphantes asiaticus van Helsdingen, 1985 & Microbathyphantes palmarius (Marples, 1955) & XXIX.ae.1 \\
\hline Lepthyphantes brincki van Helsdingen, 1985 & Nesioneta benoiti (van Helsdingen, 1978) & XXIX.aj.1 \\
\hline \multicolumn{3}{|l|}{ Family Lycosidae } \\
\hline Genus Evippella & Genus Evippa & XXXI.i. \\
\hline Genus Lycorma & Genus Hogna & XXXI.m. \\
\hline Genus Allohogna & Genus Lycosa & XXXI.n. \\
\hline Genus Avicosa & Genus Schizocosa & XXXI.t. \\
\hline Arctosa quinaria (Emerton, 1894) & Arctosa raptor (Kulczyn’ski, 1885) & XXXI.f.10 \\
\hline Hippasa pantherina Pocock, 1899 & Hippasa greenalliae (Blackwall, 1867) & XXXI.I.7 \\
\hline Ocyale neatalanta Alderweireldt, 1996 & Ocyale pilosa (Roewer, 1960) & XXXI.q.5 \\
\hline Pardosa ladakhensis Tikader, 1977 & Pardosa algoides Schenkel, 1963 & XXXI.r.1 \\
\hline Lycosa rothaka Tikader, 1970 & Pardosa fletcheri (Gravely, 1924) & XXXI.r.20 \\
\hline Pardosa annandalei Tikader \& Malhotra, 1980 & Pardosa pseudoannulata (Bösenberg \& Strand, 1906) & XXXI.r.45 \\
\hline Pardosa tatensis Tikader \& Malhotra, 1980 & Pardosa tridentis Caporiacco, 1935 & XXXI.r.63 \\
\hline \multicolumn{3}{|l|}{ Family Miturgidae } \\
\hline Cheiracathium sadanai Tikader, 1976 & Cheiracanthium melanostomum (Thorell, 1895) & XXXIII.a.13 \\
\hline \multicolumn{3}{|l|}{ Family Nephilidae } \\
\hline Herennia ornatissima (Doleschall, 1859) & Herennia multipuncta (Doleschall, 1859) & XXXVI.b.1 \\
\hline Nephila maculata (Fabricius, 1793) & Nephila pilipes (Fabricius, 1793) & XXXVI.c. 4 \\
\hline Metepeira andamanensis Tikader, 1977 & Nephilengys malabarensis (Walckenaer, 1842) & XXXVI.d.1 \\
\hline \multicolumn{3}{|l|}{ Family Psechridae } \\
\hline Psechrus alticeps Pocock, 1899 & Psechrus torvus (O.P.-Cambridge, 1869) & XLVIII.b.4 \\
\hline \multicolumn{3}{|l|}{ Family Salticidae } \\
\hline Genus Linus & Genus Portia & XLIX.by. \\
\hline Genus Oningis & Genus Jollas & XLIX.aw. \\
\hline Lyssomanes andamanensis Tikader, 1977 & Asemonea tenuipes (O.P.-Cambridge, 1869) & XLIX.c.2 \\
\hline Lyssomanes bengalensis Tikader \& Biswas, 1978 & Asemonea tenuipes (O.P.-Cambridge, 1869) & XLIX.c.2 \\
\hline Hyllus indicus (Tikader, 1974) & Hyllus semicupreus (Simon, 1885) & XLIX.ar.3 \\
\hline Myrmarachne daitarensis Prószyn'ski, 1992 & Myrmarachne plataleoides (O.P.-Cambridge, 1869) & XLIX.be.19 \\
\hline Plexippoides afghanus (Roewer, 1962) & Plexippoides flavescens (O. P.-Cambridge, 1872) & XLIX.bw.2 \\
\hline Icius icioides (Simon, 1889) & Pseudicius frigidus (O.P.-Cambridge, 1885) & XLIX.ca.7 \\
\hline Phidippus pateli Tikader, 1974 & Telamonia dimidiata (Simon, 1899) & XLIX.cs.1 \\
\hline Thyene lindbergi Roewer, 1962 & Thyene imperialis (Rossi, 1846) & XLIX.cu.1 \\
\hline
\end{tabular}




\begin{tabular}{|c|c|c|}
\hline Old name & Valid Name & Catalogue \# \\
\hline \multicolumn{3}{|l|}{ Family Sparassidae } \\
\hline Genus Torania & Genus Heteropoda & LIV.d. \\
\hline Genus Panaretus & Genus Heteropoda & LIV.d. \\
\hline Heteropoda smythiesi Simon, 1897 & Pseudopoda prompta (O.P.-Cambridge, 1885) & LIV.i.33 \\
\hline Spariolenus petricola Gravely, 1931 & Spariolenus tigris Simon, 1880 & LIV.I.2 \\
\hline Thelcticopis nigropicta Pocock, 1900 & Stasina nalandica Karsch, 1891 & LIV.m.1 \\
\hline \multicolumn{3}{|l|}{ Family Tetragnathidae } \\
\hline Genus Eucta & Genus Tetragnatha & LVII.m. \\
\hline Dyschiriognatha hawigtenera Barrion \& Litsinger, 1995 & Dyschiriognatha dentata Zhu \& Wen, 1978 & LVII.d.1 \\
\hline Tetragnatha aduncata Wang, 1991 & Tetragnatha hasselti Thorell, 1890 & LVII.m.14 \\
\hline \multicolumn{3}{|l|}{ Family Theraphosidae } \\
\hline Genus Heterophrictus & Genus Plesiophrictus & LVIII.h. \\
\hline Chilobrachys masoni (Pocock, 1895) & Chilobrachys stridulans (Wood Mason, 1877) & LVIII.b.9 \\
\hline Poecilotheria bara Chamberlin, 1917 & Poecilotheria subfusca Pocock, 1895 & LVIII.i.13 \\
\hline \multicolumn{3}{|l|}{ Family Theridiidae } \\
\hline Genus Argyrodina & Genus Argyrodes & LIX.b. \\
\hline Genus Meotipa & Genus Chrysso & LIX.e. \\
\hline Genus Trigonobothrys & Genus Dipoena & LIX.h. \\
\hline Genus Symopagia & Genus Enoplognatha & LIX.j. \\
\hline Genus Ulesanis & Genus Phoroncidia & LIX.q. \\
\hline Genus Asagena & Genus Steatoda & LIX.v. \\
\hline Genus Lithyphantes & Genus Steatoda & LIX.v. \\
\hline Genus Teutana & Genus Steatoda & LIX.v. \\
\hline Theridula caudata Saito, 1933 & Chrysso nigra (O. P.-Cambridge, 1880) & LIX.e.1 \\
\hline Euryopis taczanowskii Keyserling, 1886 & Emertonella taczanowskii (Keyserling, 1886) & LIX.i.1 \\
\hline Phoroncidia brevispinosa O. P.-Cambridge, 1873 & Phoroncidia thwaitesi O. P.-Cambridge, 1869 & LIX.q.6 \\
\hline \multicolumn{3}{|l|}{ Family Thomisidae } \\
\hline Genus Dieta & Genus Oxytate & LXI.y. \\
\hline Genus Mecostrabus & Genus Monaeses & LXI.X. \\
\hline Misumena horai Tikader, 1962 & Diaea subdola O.P.-Cambridge, 1885 & LXI.i.5 \\
\hline Misumena expallidata O. P.-Cambridge, 1885 & Ebrechtella sufflava (O. P.-Cambridge, 1885) & LXI.k.3 \\
\hline Phrynarachne nigra (O. P.-Cambridge, 1884) & Phrynarachne ceylonica (O. P.-Cambridge, 1884) & LXI.af.1 \\
\hline Thomisus cherapunjeus Tikader, 1966 & Runcinia affinis Simon, 1897 & LXI.ai.2 \\
\hline Runcinia chauhani Sen \& Basu, 1972 & Runcinia affinis Simon, 1897 & LXI.ai.2 \\
\hline Xysticus sujatai Tikader, 1962 & Xysticus croceus Fox, 1937 & LXI.as.5 \\
\hline
\end{tabular}

Note: Catalogue \# in this table refers to the number given in the South Asian Spider Checklist 9 (web supplement).

Bastawade and its transfer to family Corinnidae (Arachnida: Araneae). Zoos' Print Journal 21(7): 2307.

Benoit, P.L.G. (1964). Nouvelle contribution à la connaissance des Araneidae-Gasteracanthinae d'Afrique et de Madagascar (Araneae). Publicações culturais da Companhia de Diamanyes de Angola 69: 41-52.

Benoit, P.L.G. (1968). Synopsis des Filistatidae africains (Araneae). Annali Museo. civico Storia. naturale Giacomo Doria (Genova) 77: 92-102.

Biswas, V. \& A. Begum (1999). Jumping spiders of Bangladesh: genus Marpissa Koch (Araneae: Salticidae). Records of Zoological Survey of India 97(2): 133-138.

Biswas, V. \& D. Raychaudhuri (1996a). Clubionid spiders of Bangladesh - I: Genus Clubiona Latreille. Proceedings of recent Advances in Life Science (1994), Dibrugarh University 1: 191-210.

Biswas, V. \& D. Raychaudhuri (1996b). Tetragnathid spiders of Bangladesh (Araneae: Tetragnathidae). Annals of Entomology (Dehra Dun) 14: 45-59.

Biswas, V. \& D. Raychaudhuri (1998a). Spiders of the genus Cyclosa Menge (Araneae: Araneidae) from Bangladesh. Entomon 23: 45-53.

Biswas, V. \& D. Raychaudhuri (1998b). Jumping spiders of Bangladesh: Genus Plexippus Koch (Araneae: Salticidae). Records of zoological Survey of India 96: 167-171.

Biswas, V. \& D. Raychaudhuri (2000). Sac spiders of Bangladesh-II: Genera Castianeira Keyserling, Sphingius Thorell and Trachelas Koch (Araneae: Clubionidae). Records of Zoological Survey of India 98: 131139.

Biswas, V. \& D. Raychaudhuri (2003a). Wolf spiders of Bangladesh: genus Pardosa C. L. Koch (Araneae: Lycosidae). Records of Zoological
Survey of India 101(1-2): 107-125

Biswas, V. \& D. Raychaudhuri (2003b). Sac-spiders of Bangladesh: genus Cheiracanthium Koch (Araneae: Clubionidae). Records of Zoological Survey of India 101(3-4): 115-124.

Biswas, V. \& D. Raychaudhuri (2003c). A new species of spider of the genus Tibellus Simon (Araneae: Thomisidae) from Jhenidah, Bangladesh. Journal of the Bombay Natural History Society 100: 84-86.

Biswas, V. \& D. Raychaudhuri (2004). New orb-weaving spiders of the genus Cyrtophora Simon (Araneae: Araneidae) from Bangladesh. Journal of the Bombay Natural History Society 101: 124-129.

Biswas, V. (1999). Description of two new species of jumping spiders (Araneae: Salticidae) of the genera Phidippus Koch and Plexippus Koch from Bangladesh. Entomon 24: 331-337.

Bonaldo, A.B. (2000). Taxonomia da subfamília Corinninae (Araneae, Corinnidae) nas regiões Neotropica e Neárctica. Iheringia (Zoology) 89: 3-148.

Bonaldo, A.B. \& A.D. Brescovit (1997). Uma nova espécie do gênero Oltacloea (Araneae: Prodidomidae). Iheringia (Zoology) 82: 81-84.

Brignoli, P.M. (1972). Spinnen aus Nepal, I. Paculla martensi n. sp. (Arachnida: Araneae: Pacullidae). Senckenbergiana biologica 53: 95-100. Brignoli, P.M. (1973). Spinnen aus Nepal, II. Zur Morphologie der Gattung Althepus Thorell, nebst Beschreibung zweier neuer Arten (Arachnida: Araneae: Ochyroceratidae). Senckenbergiana biologica 54: 157164

Brignoli, P.M. (1976a). Beiträge zur Kenntnis der Scytodidae (Araneae). Revue suisse de Zoologie 83: 125-191.

Brignoli, P.M. (1976b). Spinnen aus Nepal, III. Über einige Spinnen aus 
dem Himalaya, dazu Revision einiger Arten aus dem Karakorum (Arachnida: Araneae). Ergebn. ForschUnternehmens Nepal Himalaya 5: 229253.

Brignoli, P.M. (1978). Ergebnisse der Bhutan-Expedition 1972 des Naturhistorischen Museums in Basel. Araneae: Fam. Oonopidae, Agelenidae, Hahniidae und Mimetidae. Entomologisto brasileiro (Sao Paulo) 3: 31-56.

Brignoli, P.M. (1979). On some African Oecobius and Zimiris (Araneae, Oecobiidae and Gnaphosidae). Zoologische mededeelingen. Leiden 54: 123126.

Brignoli, P.M. (1980). On few Mysmenidae from the Oriental and Australian regions (Araneae). Revue suisse Zool. 87: 727-738.

Brignoli, P.M. (1981). New or interesting Anapidae (Arachnida, Araneae). evue suisse de Zoologie 88: 109-134.

Brignoli, P.M. (1982). On a few spiders from China (Araneae). Bulletin of the British Arachnological Society 5: 344-351.

Brignoli, P.M. (1983). A Catalogue of the Araneae Described between 1940 and 1981. Manchester University Press, 755pp.

Brignoli, P.M. (1986). A new Simonicera (Araneae: Ochyroceratidae) from Guam, Marianas. Boll. Mus. civ. Stor. nat. Verona 11: 345-348.

Buchar, J. \& K. Thaler (1993). Die Arten der Gattung Acantholycosa in Westeuropa (Arachnida, Araneida: Lycosidae). Revue suisse Zoology 100: 327-341.

Buchar, J. (1976). Über einige Lycosiden (Araneae) aus Nepal. Ergebn. ForschUnternehmens Nepal Himalaya 5: 201-227.

Buchar, J. (1997). Lycosidae aus Bhutan 1. Venoniinae und Lycosinae (Arachnida: Araneae). Entomologisto brasileiro (Sao Paulo) 20: 5-32.

Caporiacco, L.di. (1935). Aracnidi dell'Himalaia e del Karakoram, raccolti dalla Missione italiana al Karakoram (1929-VII). Mem. Soc. ent. ital. 13: 161-263.

Chamberlin, R.V. \& W. Ivie (1942). A hundred new species of American spiders. Bulletin of the University of Utah 32(13): 1-117.

Sadana, G.L. \& M. Kaur (1974). A new species of Chorizopes O.P.-C. (Araneida: Argiopidae) from India. Entomologist's monthly magazine 109: 162-163.

Coyle, F.A. (1995). A revision of the funnel web mygalomorph spider subfamily Ischnothelinae (Araneae: Dipluridae). Bulletin of the American Museum of Natural History 226: 1-133.

Crosby, C.R. (1905). A catalogue of the Erigoneae of North America, with notes and descriptions of new species. Proceedings of the Academy of Natural Sciences of Philadelphia 57: 301-343.

Dalmas, R.de. (1920). Liste d'araignées de Boudron en Asie Mineure suive d'une étude des espèces méditerranéennes du genre Habrocestum. Annali del Musea civico di storia naturale di Genova 50: 57-69.

Davies, V.T. (1985). Araneomorphae (in part). Zoological catalogue of Australia 3: 49-125.

Davies, V.T. (1994). The huntsman spiders Heteropoda Latreille and riinthi gen. nov. (Araneae: Heteropodidae) in Australia. Memoires of Queensland Musuem 35: 75-122.

Deeleman-Reinhold, C.L. \& P.R. Deeleman (1988). Revision des Dysderinae (Araneae: Dysderidae), les especes mediterraneennes occidentales exceptees. Tijdschrift voor Entomologie 131: 141-269.

Deeleman-Reinhold, C.L. (1993). A new spider genus from Thailand with a unique ant-mimicking device, with description of some other castianeirine spiders (Araneae: Corinnidae: Castianeirinae). Natural history bulletin of the Siam Society (Bangkok) 40: 167-184.

Deeleman-Reinhold, C.L. (1995). The Ochyroceratidae of the IndoPacific region (Araneae). The Raffles Bulletin of Zoology Supplement number2: 1-103.

Deeleman-Reinhold, C.L. (2001). Forest spiders of South East Asia: with a revision of the sac and ground spiders (Araneae: Clubionidae, Corinnidae, Liocranidae, Gnaphosidae, Prodidomidae and Trochanterriidae [sic]). Brill, Leiden, $591 \mathrm{pp}$

Denis, J. (1958). Araignées (Araneidea) de l'Afghanistan. I. Vidensk. Meddr dansk naturh. Foren. 120: 81-120.

Dondale, C. D. \& J. H. Redner (1983). Revision of the wolf spiders of the genus Arctosa C.L. Koch in North and Central America (Araneae: Lycosidae). Journal of Arachnology 11: 1-30.
Dyal, S. (1935). Spiders of Lahore. Bulletin of Department of Zoology, Punjab University 1: 117-252.

Dyal, S. (1957). A new genus of the spiders of the family Sparassidae. Res. Bulletin of Department of Zoology, Panjab University 134: 561-566.

Edmunds, M. \& J. Prószyn'ski (2003). On a collection of Myrmarachne spiders (Araneae: Salticidae) from peninsular Malaya. Bulletin of the British Arachnological Society 12: 297-323.

Emerit, M. (1974). Arachnides araignées Araneidae Gasteracanthinae. Faune Madagascar 38: 1-215.

Fabricius, J.C. (1798). Araneae, pp.291-294. Supplementum entomologiae systematicae. Hafniae, 572pp.

Fitzgerald, B.M. \& P.J. Sirvid (2003). The genus Trigonobothrys in New Zealand and a redescription of Achaearanea blattea (Theridiidae: Araneae). Tuhinga 14: 25-33.

Fuhn, I.E. \& F. Niculescu-Burlacu (1971). Fam. Lycosidae. Fauna Republicii Socialiste România (Arachnida) 5(3): 1-253.

Galiano, M.E. (1991). Revision del género Jollas (Araneae: Salticidae). Physis B. Aires (C) 47: 15-29.

Grasshoff, M. (1970). Die Tribus Mangorini. I. Die Gattungen Eustala, Larinia s. str., Larinopa n. gen. (Arachnida: Araneae: Araneidae-Araneinae). Senckenbergiana biologica 51: 209-234.

Grasshoff, M. (1971). Die Tribus Mangorini, IV. Die Mangora-Gruppe (Arachnida: Araneae: Araneidae-Araneinae). Senckenbergiana biologica 52: 293-311.

Grasshoff, M. (1976). Zur Taxonomie und Nomenklatur mitteleuropäischer Radnetzspinnen der Familie Araneidae (Arachnida: Araneae). Senckenbergiana biologica 57: 143-154.

Grasshoff, M. (1986). Die Radnetzspinnen-Gattung Neoscona in Afrika (Arachnida: Araneae). Zoologische Wetenschaft 250: 1-123.

Gravely, F.H. (1931). Some Indian spiders of the families Ctenidae, Sparassidae, Selenopidae and Clubionidae. Records of Indian Museum, Calcutta 33: 211-282.

Harm, M. (1981). Revision der mitteleuropäischen Arten der Gattung Marpissa C.L. Koch 1846 (Arachnida: Araneae: Salticidae).Senckenbergiana biologica 61: 277-291.

Heciak, S. \& J. Prószyn'ski (1983). Remarks on Langona Simon (Araneae, Salticidae). Annales zoologici Warszawa 37: 207-233.

Helsdingen, P.J. van. (1979). Remarks on Nematogmus dentimanus Simon, with comments on the status of related genera (Araneae: Erigonidae). Bulletin of British arachnological Society 4: 407-413.

Helsdingen, P.J. van. (1985). Araneae: Linyphiidae of Sri Lanka, with a note on Erigonidae. Entomologica Scandinavica (Suppl.) 30: 13-30.

Hentz, N.M. (1850). Descriptions and figures of the araneides of the United States. Boston ournal of the Natural History 6: 18-35, 271-295.

Hippa, H. \& P.T. Lehtinen (1983). The Zantheres group of Zoicinae (Araneae, Lycosidae) and a relimitation of the subfamily. Annales Zoologici Fennici 20: 151-156.

Homann, H. (1975). Die Stellung der Thomisidae und der Philodromidae im System der Araneae (Chelicerata, Arachnida). Zeitschrift für Morphologie der Tiere 80: 181-202.

Hormiga, G. \& N. Scharff (2005). Monophyly and phylogenetic placement of the spider genus Labulla Simon, 1884 (Araneae, Linyphiidae) and description of the new genus Pecado. Zoological Journal of the Linnean Society 143: 359-404.

Hu, J.L. (2001). Spiders in Qinghai-Tibet Plateau of China. Henan Science and Technology Publishing House, 658pp.

Jäger, P. (2000). Two new heteropodine genera from southern continental Asia (Araneae: Sparassidae). Acta arachnologica, Tokyo 49: 61-71.

Jäger, P. (2001). Diversität der Riesenkrabbenspinnen im Himalaya die Radiation zweier Gattungen in den Schneetropen (Araneae, Sparassidae, Heteropodinae). Cour. Forsch.-Inst. Senckenberg 232: 1-136.

Jäger, P. (2002). Heteropodinae: transfers and synonymies (Arachnida: Araneae: Sparassidae). Acta arachnologica, Tokyo 51: 33-61.

Jäger, P. (2005). New large-sized cave-dwelling Heteropoda species from Asia, with notes on their relationships (Araneae: Sparassidae: Heteropodinae). Revue Suisse de Zoologie 112: 87-114.

Jäger, P. (2006). Martensopoda gen. nov. from southern Indian mountain ranges, the first genus of huntsman spiders with a cymbial spur (Araneae: 
Sparassidae: Heteropodinae). Zootaxa 1325: 335-345.

Jäger, P., J.C. Gao \& R.I. Fei (2002). Sparassidae in China 2. Species from the collection in Changchun (Arachnida: Araneae). Acta arachnologica, Tokyo 51: 23-31.

Jocqué, R. (1986a). A revision of the genus Hermippus Simon, 1893 (Araneae: Zodariidae). Journal of Natural History 20: 7-22.

Jocqué, R. (1986b). Ant-eating spiders from the Comoros (Araneae, Zodariidae). Revue zoologique africaine (Bruxelles et Paris) 100: 307-312.

Jocqué, R. (1991). A generic revision of the spider family Zodariidae (Araneae). Bulletin of the American Museum of Natural History 201: 1-160. Jocqué, R. \& R. Bosmans (1989). A revision of the genus Storenomorpha Simon (Araneae, Zodariidae). Spixiana 12: 125-134.

Karsch, E. (1891). Arachniden von Ceylon und von Minikoy gesammelt von den Herren Doctoren P. und F. Sarasin. Berliner Entomologische Zeitschrift 36: 267-310.

Kirk, P.J. (1996). A new species of Poecilotheria (Araneae: Theraphosidae) from Sri Lanka. British Tarantula Society Journal 12: 20-30.

Koch, C.L. (1846). Die Arachniden. Nürnberg, Dreizehnter Band, pp. 1234, Vierzehnter Band, pp.1-88.

Kraus, O. \& M. Kraus (1989). The genus Stegodyphus (Arachnida, Araneae). Sibling species, species groups, and parallel origin of social living. Verhandlungen naturwissenschaftlichen Vereins, Hamburg 30: 151-254.

Kronestedt, T. (1993). Species of Wadicosa (Araneae: Lycosidae): revised generic allocation of Lycosa quadrifer Gravely from Sri Lanka and India. Journal of Natural History 27: 313-321.

Kuntner, M. (2005). A revision of Herennia (Araneae: Nephilidae: Nephilinae), the Australasian 'coin spiders'. Invertebrate Systematics 19: $391-436$.

Kuntner, M. (2006). Phylogenetic systematics of the Gondwanan nephilid spider lineage Clitaetrinae (Araneae: Nephilidae). Zoologica Scripta 35: 19-62.

Kurata, T.B. (1944). Two new species of Ontario spiders. Occasional papers of the Royal Ontario Museum of Zoology 8: 1-6.

Lee, C.L. (1966). [Spiders of Formosa (Taiwan)]. Taichung Jun. Teachers Coll. Publ., 84pp.

Lehtinen, P.T. (1967). Classification of the cribellate spiders and some allied families, with notes on the evolution of the suborder Araneomorpha. Annales Zoologici Fennici 4: 199-468.

Lehtinen, P.T. (2005). Taxonomic notes on the Misumenini (Araneae: Thomisidae: Thomisinae), primarily from the Palaearctic and Oriental regions. In: Logunov, D. V. \& D. Penney (Eds.), European Arachnology 2003 (Proceedings of the 21 st European Colloquium of Arachnology, St.-Petersburg, 4-9 August 2003). Arthropoda Selecta, Special Issue 1: 147184.

Lehtinen, P.T. \& H. Hippa (1979). Spiders of the Oriental-Australian region I. Lycosidae: Venoniinae and Zoicinae. Annales Zoologici Fennici 16: $1-22$.

Lehtinen, P.T. \& M.I. Saaristo (1980). Spiders of the Oriental-Australian region. II. Nesticidae. Annales Zoologici Fennici 17: 47-66.

Lessert, R. (1927). de. Araignées du Congo (Premiere partie). evue suisse de Zoologie 34: 405-475.

Levi, H.W. (1957). The spider genera Crustulina and Steatoda in North America, Central America, and the West Indies (Araneae: Theridiidae). Bulletin of the Museum of Comparative Zoology, Harvard 117: 367-424.

Levi, H.W. (1959). The spider genus Latrodectus (Araneae: Theridiidae). Transactions of the American Microscopical Society 78: 7-43.

Levi, H.W. (1981). The American orb-weaver genera Dolichognatha and Tetragnatha north of Mexico (Araneae: Araneidae, Tetragnathinae). Bulletin of the Museum of Comparative Zoology, Harvard 149: 271-318.

Levi, H.W. (1982). The spider genera Psechrus and Fecenia (Araneae: Psechridae). Pacific Insects 24: 114-138.

Levi, H.W. (1983). The orb-weaver genera Argiope, Gea, and Neogea from the western Pacific region (Araneae: Araneidae, Argiopinae). Bulletin of the Museum of Comparative Zoology, Harvard 150: 247-338.

Levi, H.W. (1995). Orb-weaving spiders Actinosoma, Spilasma, Micrepeira, Pronous, and four new genera (Araneae: Araneidae). Bulletin of the Museum of Comparative Zoology, Harvard, 154: 153-213.

Levi, H.W. \& L.R. Levi (1962). The genera of the spider family
Theridiidae. Bulletin of the Museum of Comparative Zoology, Harvard 127: $1-71$.

Levy, G. (1999). Spiders of six uncommon drassodine genera (Araneae: Gnaphosidae) from Israel. Israel Journal of Zoology 45: 427-452.

Levy, G. (2004). Spiders of the genera Drassodes and Haplodrassus (Araneae: Gnaphosidae) from Israel. Israel Journal of Zoology 50: 1-37.

Logunov, D.V. \& M. Zamanpoore (2005). Salticidae (Araneae) of Afghanistan: an annotated check-list, with descriptions of four new species and three new synonymies. Bulletin of British arachnological Society 13: 217-232

Logunov, D.V. (2001). A redefinition of the genera Bianor Peckham \& Peckham, 1885 and Harmochirus Simon, 1885, with the establishment of a new genus Sibianor gen. n. (Aranei: Salticidae). Arthropoda Selecta 9: 221-286.

Logunov, D.V. (2004). On the taxonomic position of "Lyssomanes" karnatakaensis and other Indian species formerly assigned to Lyssomanes (Araneae: Salticidae). Bulletin of the British Arachnological Society 13: 7375 .

Lucas, H. (1846). Histoire naturelle des animaux articules. In: Exploration scientifique de l'Algerie pendant les annees 184.0, 184.1, 184.2 publiee par ordre du Gouvernement et avec le concours d'une commission academique. Paris, Sciences physiques, Zoologie, 1: 89-271.

Marusik, Y.M. (1991). Spider genus Chalcoscirtus (Aranei, Salticidae) from the USSR. Communication 2. Zoologisches Zentralblatt (Leipzig) 70(1): 19-31.

Marusik, Y.M. (1993). Re-description of spiders of the families Heteropodidae and Thomisidae (Aranei), described by O.P.-Cambridge from the material of the second Yarkand mission. Entomologicheskoe obozrenie 72: 456-468.

Mello-Leitão, C.F. de. (1946). Notas sobre os Filistatidae e Pholcidae. Anais da Academia brasileira de ciencias (Rio da Janeiro) 18: 39-83.

Millidge, A.F. (1977). The conformation of the male palpal organs of linyphiid spiders, and its application to the taxonomic and phylogenetic analysis of the family (Araneae: Linyphiidae). Bulletin of British Arachnological Society 4: 1-60.

Millidge, A.F. (1995). Some linyphiid spiders from south-east Asia. Bulletin of British arachnological Soceity 10: 41-56.

Molur, S. \& M. Siliwal (2004). Common names of South Asian theraphosid spiders (Araneae: Theraphosidae). Zoos' Print Journal 19(10): 1657-1662.

Nenilin, A.B. (1984a). Materials on the fauna of the spider family Salticidae of the USSR. I. Catalog of the Salticidae of central Asia, pp.637. In: Fauna and Ecology of Arachnids. University of Perm.

Nenilin, A.B. (1984b). On the taxonomy of spiders of the family Salticidae of the fauna of the USSR and adjacent countries. Zoologisches Zentralblatt (Leipzig) 43: 1175-1180.

Okuma, C. (1983). New synonymies and new records of some cosmopolitan species of the genus Tetragnatha (Araneae: Tetragnathidae). Esakia 20: 69-80.

Olivier G.A. (1789). Araignée, Aranea. Encycl. méth. Hist. nat. Ins. Paris 4: 173-240.

Ono, H. (1978). Thomisidae aus dem Nepal-Himalaya. I. Das Genus Xysticus C. L. Koch 1835 (Arachnida: Araneae). Senckenbergiana biologica 59: 267-288

Ono, H. (1979). Thomisidae aus dem Nepal-Himalaya. II. Das Genus Lysiteles Simon 1895 (Arachnida: Araneae). Senckenbergiana biologica 60: 91-108.

Ono, H. (1980). Thomisidae aus dem Nepal-Himalaya. III. Das Genus Stiphropus Gerstaecker 1873, mit Revision der asiatischen Arten (Arachnida: Araneae). Senckenbergiana biologica 61: 57-76.

Ono, H. (1983). Zodariidae aus dem Nepal-Himalaya. I. Neue Arten der Gattung Storena Walckenaer 1805 (Arachnida: Araneae). Senckenbergiana biologica 63: 211-217.

Ono, H. (1985). Revision einiger Arten der Familie Thomisidae (Arachnida, Araneae) aus Japan. Bulletin of National Science Museum of Tokyo (A) 11: 19-39.

Ono, H. (1988). A Revisional Study of The Spider Family Thomisidae (Arachnida: Araneae) of Japan. National Science Museum, Tokyo, ii+252pp. 
Ono, H. (2000). Zoogeographic and taxonomic notes on spiders of the subfamily Heptathelinae (Araneae, Mesothelae, Liphistiidae). Memoirs of the National Science Museum of Tokyo (A) 33: 145-151.

Ovtsharenko, V.I. \& V.Y. Fet (1980). Fauna and ecology of spiders (Aranei) of Badhyz (Turkmenian SSR). Entomologicheskoe obozrenie 59: 4.42-447.

Patel, B.H. (1978). Studies on Indian filistatid spiders (Araneae: Arachnida). Journal of the Bombay Natural History Society 75: 183-189.

Peckham, G.W. \& E.G. Peckham (1888). Attidae of North America. Transactions of the Wisconsin Academy of Sciences, Arts and Letters 7: 1-104. Platnick, N.I. (1985). Studies on Malagasy spiders, 2. The family Trochanteriidae (Araneae: Gnaphosoidea), with a revision of the genus Platyoides. American Museum Novitates 2808: 1-17.

Platnick, N.I. (1989). Advances in Spider Taxonomy 1981-1987: A Supplement to Brignoli's A Catalogue of the Araneae described between 1940 and 1981. Manchester Univ. Press, 673pp.

Platnick, N.I. (1990). Spinneret morphology and the phylogeny of ground spiders (Araneae: Gnaphosoidea). American Museum Novitates 2978: $1-42$.

Platnick, N. I. (2006). The world spider catalog, version 7.0. American Museum of Natural History, online at http://research.amnh.org/ entomology/spiders/catalog/index.html

Platnick, N.I. \& B. Baehr. (2006). A revision of the Australasian ground spiders of the family Prodidomidae (Araneae, Gnaphosoidea). Bulletin of American Museum Natural History 298: 1-287.

Platnick, N.I., J.A. Coddington, R.R. Forster \& C.E. Griswold (1991). Spinneret morphology and the phylogeny of haplogyne spiders (Araneae, Araneomorphae). American Museum Novitates 3016: 1-73.

Platnick, N.I. \& J.A. Murphy (1984). A revision of the spider genera Trachyzelotes and Urozelotes (Araneae, Gnaphosidae). American Museum Novitates 2792: 1-30.

Platnick, N.I. \& J.A. Murphy (1996). A review of the zelotine ground spider genus Setaphis (Araneae: Gnaphosidae). American Museum Novitates 3162: 1-23.

Platnick, N.I. \& M.U. Shadab (1974). A revision of the spider family Stenochilidae (Arachnida: Araneae). American Museum Novitates 2556: 114.

Platnick, N.I. \& V.I. Ovtsharenko (1995). An Australian ground spider of the genus Zelotes (Araneae: Gnaphosidae). Records of the Western Australian Museum Supplement 52: 131-133.

Pocock, R.I. (1895). Notes on the identity of some of the types of Mygalomorphae in the collection of the British Museum. Annals and Magazine of Natural History (6)16: 223-230.

Pocock, R.I. (1900). The fauna of British India, including Ceylon and Burma. Arachnida. London, 279pp.

Prószyn'ski, J. (1971). Catalogue of Salticidae (Aranei) specimens kept in major collections of the world. Annales zoologici Warszawa 28: 367519.

Prószyn'ski, J. (1975). Remarks on the origin and composition of the Salticidae fauna of the Nearctic region. Proceeding of 6th international arachnological Congress, pp. 216-221.

Prószyn'ski, J. (1976). Studium systematyczno-zoogeograflczne nad rodzina Salticidae (Aranei) Regionów Palearktycznego i Nearktycznego. Wyzsza Szkola Pedagogiczna Siedlcach 6: 1-260.

Prószyn'ski, J. (1984a). Atlas rysunków diagnostycznych mniej znanych Salticidae (Araneae). Wyzsza Szkola Rolniczo-Pedagogiczna, Siedlcach 2: 1177.

Prószyn'ski, J. (1984b). Remarks on Anarrhotus, Epeus and Plexippoides (Araneae, Salticidae). Annales zoologici Warszawa 37: 399-410.

Prószyn'ski, J. (1984c). Remarks on Viciria and Telamonia (Araneae, Salticidae). Annales zoologici Warszawa 37: 417-436.

Prószyn'ski, J. (1987). Atlas rysunkow diagnostycznych mniej znanych Salticidae 2. Zeszyty Naukowe Wyzszej Szkoly Rolniczo-Pedagogicznej, Siedlcach.

Prószyn'ski, J. (1990). Catalogue of Salticidae (Araneae): Synthesis of Quotations in the World Literature since 1940, with Basic Taxonomic Data since 1758. Wyzsza Szkola Rolniczo-Pedagogiczna W Siedlcach, 366pp.

Prószyn'ski, J. (1992). Salticidae (Araneae) of India in the collection of the Hungarian National Natural History Museum in Budapest. Annales zoologici Warszawa 44: 165-277.

Prószyn'ski, J. (1999). Description of Rafalus gen. n. (Aranei: Salticidae), with special reference to the Near East fauna. Arthropoda Selecta 8: 89101.

Prószyn’ski, J. \& W. Starega (1971). Pajaki-Aranei. Kat. Fauny polski 33: $1-382$.

Raven, R.J. (1980). The evolution and biogeography of the mygalomorph spider family Hexathelidae (Araneae, Chelicerata). Journal of Arachnology 8: 251-266.

Raven, R.J. (1985). The spider infraorder Mygalomorphae (Araneae): Cladistics and systematics. Bulletin of the American Museum of Natural History 182: 1-180.

Raven, R.J. (1986). A revision of the spider genus Sason Simon (Sasoninae, Barychelidae, Mygalomorphae) and its historical biogeography. Journal of Arachnology 14: 47-70.

Reimoser, E. (1919). Katalog der echten Spinnen (Araneae) des Paläarktischen Gebietes. Abhandlungen des Zoologisches und Botanisches Gesellschaft von Wien 10(2): 1-280.

Roberts, M.J. (1983). Spiders of the families Theridiidae, Tetragnathidae and Araneidae (Arachnida: Araneae) from Aldabra atoll. Zoological Journal of the Linnean Society 77: 217-291.

Roewer, C.F. (1942). Katalog der Araneae von 1758 bis 1940. Bremen, 1: 11040 .

Roewer, C.F. (1951). Neue Namen einiger Araneen-Arten. Abhandlungen hrsg. vom naturwissenschaftlichen Verein $\approx u$ Bremen 32: 437-456.

Roewer, C.F. (1955). Katalog der Araneen von 1758 bis 194.O, bzw. 1954. Bruxelles, 2: 1-1751.

Roewer, C. F. (1959). Araneae Lycosaeformia II (Lycosidae). Explor. Parc natn. Upemba Miss. G. F. de Witte 55: 1-518.

Roewer, C.F. (1960a). Solifugen und Opilioniden - Araneae Orthognathae, Haplogynae und Entelegynae (Contribution à l'étude de la faune d'Afghanistan 23). Göteborgs K. Vetensk.-o. VitterhSamh. Handl. 8(7): 1-53. Roewer, C.F. (1960b). Lycosidae aus Afghanistan (Araneae). Acta Univ. Lundensis (N.F.) (2)56(17): 1-34.

Roewer, C.F. (1961). Araneae Dionycha aus Afghanistan I. Acta Univ. Lundensis (N.F.) (2)58(3): 1-33.

Roewer, C.F. (1962). Araneae Dionycha aus Afghanistan II. AActa Univ. Lundensis (N.F.) (2)58(4): 1-34.

Roth, V.D. (1967). Descriptions of the spider families Desidae and Argyronetidae. American Museum Novitates 2292: 1-9.

Saaristo, M.I. (1995). Linyphiid spiders of the granitic islands of Seychelles (Araneae, Linyphiidae). Phelsuma 3: 41-52.

Saaristo, M.I. (1996). Theridiosomatid spiders of the granitic islands of Seychelles (Araneae, Theridiosomatidae). Phelsuma 4: 48-52.

Saaristo, M.I. \& A.V. Tanasevitch (1996). Redelimitation of the subfamily Micronetinae Hull, 1920 and the genus Lepthyphantes Menge, 1866 with descriptions of some new genera (Aranei, Linyphiidae). Ber. nat.-med. Verein Innsbruck 83: 163-186.

Saaristo, M.I. \& A.V. Tanasevitch (1999). Reclassification of the mughigroup of the genus Lepthyphantes Menge, 1866 (sensu lato) (Araneae: Linyphiidae: Micronetinae). Ber. nat.-med. Verein Innsbruck 86: 139-147. Saaristo, M.I. \& A.V. Tanasevitch (2003). A new micronetid spider genus from the Oriental Region (Aranei: Linyphiidae: Micronetinae). Arthropoda Selecta 11: 319-330.

Saha, S., V. Biswas \& D. Raychaudhuri (1995). A new name for Heteropoda acuta Saha, Biswas and Raychaudhuri, 1994. (Araneae: Heteropodidae). Acta arachnologica, Tokyo 44: 15-16.

Schmidt, G.E.W. (2004). Eine zweite Art der Gattung Haplocosmia Schmidt \& von Wirth, 1996 (Araneae: Theraphosidae: Selenocosmiinae). Tarantulas of the World 99: 5-7.

Sethi, V.D. \& B.K. Tikader. (1988). Studies on some giant crab spiders of the family Heteropodidae from India. Records of Zoological Survey of India, Occasional Paper 93: 1-94.

Shear, W.A. (1970). The spider family Oecobiidae in North America, Mexico, and the West Indies. Bulletin of the Museum of Comparative Zoology, Harvard 140: 129-164.

Shear, W.A. (1978). Taxonomic notes on the armored spiders of the 
families Tetrablemmidae and Pacullidae. American Museum Novitates 2650: $1-46$.

Sierwald, P. (1987). Revision der Gattung Thalassius (Arachnida, Araneae, Pisauridae). Verhandlungen naturwissenschaftlichen Vereins, Hamburg 29: 51142 .

Sierwald, P. (1989). Genital morphology of the genus Perenethis (Araneae: Pisauridae). Report of the Department of Biology, University of Turku 19: 97

Siliwal, M., S. Molur \& B.K. Biswas (2005). Indian spiders (Arachnida, Araneae): updated checklist 2005. Zoos' Print Journal 20(10): 1999-2049. Simon, E. (1876). Les arachnides de France. Paris, 3: 1-364.

Simon, E. (1880). Révision de la famille des Sparassidae (Arachnides). Actes de la Sociètè linnèenne de Bordeaux 34: 223-351.

Simon, E. (1884). Les arachnides de France. Paris, 5: 180-885.

Simon, E. (1885). Matériaux pour servir à la faune arachnologiques de l'Asie méridionale. III. Arachnides recuellis en 1884 dans la presqu'île de Malacca, par M.J. Morgan. IV. Archnides recuellis à Collegal, district de Coimbatoore, par M.A. Theobald G.R. Bull. Soc. zool. France 10: 436-462. Simon, E. (1886). Espèces et genres nouveaux de la famille des Thomisisdae. Act. Soc. linn. Bord. 40: 167-187.

Simon, E. (1887). Etudes arachnologiques. 19e Mémoire. XXVII. Arachnides recuellis à Assinie (Afrique occidentale) par MM. Chaper et Alluaud. Annales de la Sociètè entomologique de France (6) 7: 261-276.

Simon, E. (1889). Arachnides. In Voyage de M. E. Simon au Venezuela (décembre 1887-avril 1888). 4e Mémoire. Annales de la Sociètè entomologique de France 6(9): 169-220.

Simon, E. (1893a). Histoire naturelle das araignées. Paris, 1: 257-488.

Simon, E. (1893b). Etudes arachnologiques. 25e Mémoire. XL Descriptiones d'espèces et de genres nouveaux de l'ordre des Araneae. nnales de la Sociètè entomologique de France 62: 299-330.

Simon, E. (1894). Histoire naturelle des araignées. Paris, 1: 489-760.

Simon, E. (1895). Histoire naturelle des araignées. Paris, 1: 761-1084.

Simon, E. (1896). Descriptions d'arachnides nouveaux de la famille des Clubionidae. Annals of the Society of Entomology Belgium 40: 400-422.

Simon, E. (1897a). Matériaux pour servir à la faune arachnologique de l'Asie méridionale. V. Arachnides recueillis à Dehru-Dun (N. W. Prov.) et dans le Dekken par M.A. Smythies. Mémoires de la Société zoologique de France 10: 252-262.

Simon, E. (1897b). Arachides recueillis par M. M. Maindron à Kurrachee et à Matheran (près Bombay) en 1896. Bulletin du Museum (national) d'histoire naturelle, Paris 1897: 289-297.

Simon, E. (1898). Histoire naturelle des araignées. Paris, 2: 193-380.

Simon, E. (1900). Arachnida. In Fauna Hawaiiensis, or the zoology of the Sandwich Isles: being results of the explorations instituted by the Royal Society of London promoting natural knowledge and the British Association for the Advancement of Science. London, 2: 443-519.

Simon, E. (1901). Etudes arachnologiques. 31e Mémoire. L. Descriptions d'espèces nouvelles de la famille des Salticidae (suite). Annales de la Sociètè entomologique de France 70: 66-76.

Simon, E. (1902). Etudes arachnologiques. 31 e Mémoire. LI. Descriptions d'espèces nouvelles de la famille des Salticidae (suite). Annales de la Sociètè entomologique de France 71: 389-421.

Simon, E. (1903). Histoire naturelle des araignées. Paris, 2: 669-1080.

Simon, E. (1904). Arachnides recueillis par M.A. Pavie en Indochine. In Mission Pavie en Indochine 1879-1895. III. Recherches sur l'histoire naturells de l'Indochine Orientale. Paris, pp. 270-295.

Simon, E. (1905). Arachnides des îles Chatham. (Ergebnisse einer Reise nach dem Pacific. Schauinsland 1896-1897). Zool. Jahrb. Syst. 21: 415424.

Simon, E. (1906). Etude sur les araignées de la section des cribellates. Annals of the Society of Entomology Belgium 50: 284-308.

Simon, E. (1907). Araneae, Chernetes et Opiliones (Première série). In Biospeologica. III. Arch. zool. expér. gen. (4) 6: 537-553.

Sinha, T.B. (1951). Some Indian spiders of the family Hersiliidae. Records of Indian Museum 48: 121-126.

Sivaperuman, C., M. Karthikeyan \& R. Ravikumar (2005). Diversity of spiders in Parambikulam Wildlife Sanctuary. Tigerpaper 32(4): 18-23. Smith, A.M. (1986). Species file: Rhechostica seemanni (Cambridge, 1897).
Journal of the British Tarantula Society 1: 22-26.

Smith, A.M. \& P. Kirk (2001). A Field Guide on the Theraphosid Spiders of Indian and Sri Lanka particularly the Genus Poecilotheria. (unpublished).

Song, D.X. (1987). Spiders from agricultural regions of China (Arachnida: Araneae). Agriculture Publishing House, Beijing.

Song, D.X., Z.Q. Feng \& J.W. Shang (1982). On the males of two species of crab spiders (Araneida: Thomisidae). Acta zootaxonomia sinica 7: 257-259

Strand, E. (1928). Die arachnologischen Gattungsnamen Archaea und Argyope. Ent. Nachrbl. Troppau 2: 46

Strand, E. (1934). Miscellanea nomenclatorica zoologica et palaeontolgica, VI. Folia zool. hydrobiol. 6: 271-277.

Taczanowski, L. (1872). Les aranéides de la Guyane française. Horae Soc. ent. Ross. 9: 64-112.

Tanasevitch, A.V. (1998a). Gorbothorax n. gen., a new linyphiid spider genus from the Nepal Himalayas (Arachnida: Araneae: Linyphiidae). Bonner Zoologische Beiträge 47: 42 1-428.

Tanasevitch, A.V. (1998b). New Oedothorax Bertkau, 1883, from Nepal (Arachnida, Araneae, Linyphiidae). Bonner Zoologische Beiträge 47: 429441 .

Tanasevitch, A.V. (1992). New genera and species of the tribe Lepthyphantini (Aranei Linyphiidae Micronetinae) from Asia (with some nomenclatorial notes on linyphiids). Arthropoda Selecta 1(1): 39-50.

Tanikawa, A. \& H. Ono (1993). Spiders of the genus Cyclosa (Araneae, Araneidae) from Taiwan. Bulletin of the National Science Museum. Tokyo (A) 19: 51-64.

Tikader, B.K. (1970). Spider fauna of Sikkim. Records of Zoological Survey of India 64: 1-83.

Tikader, B.K. (1971). Revision of Indian crab spiders (Araneae: Thomisidae). Memoires of the Zoological Survey of India 15(8): 1-90.

Tikader, B.K. (1976). Redescription of a jumping spider Harmochirus brachiatus (Thorell) with a new record from India. Journal of the Bombay Natural History Society 73: 410-411.

Tikader, B.K. (1977). Studies on spider fauna of Andaman and Nicobar islands, Indian Ocean. Records of Zoological Survey of India 72: 153-212. Tikader, B.K. (1982a). Family Araneidae (=Argiopidae), typical orbweavers. Fauna India (Araneae) 2: 1-293.

Tikader, B.K. (1982b). Family Gnaphosidae. Fauna India (Araneae) 2: 295-536.

Tikader, B.K. (1987). Handbook of Indian Spiders (Anon, Ed.). Zoological Survey of India, Calcutta, $251 \mathrm{pp}$.

Tikader, B.K. \& A. Bal (1981). Studies on spiders of the genus Zygiella Cambridge from India (Araneae: Araneidae). Proceedings of Indian Academy of Science (Anim. Sci.) 89: 243-246.

Tikader, B.K. \& B. Biswas (1981). Spider fauna of Calcutta and vicinity: Part-I. Records of Zoological Survey of India, Occasional Paper 30: 1-149. Tikader, B.K. \& M.S. Malhotra (1980). Lycosidae (wolf-spiders).

Fauna India (Araneae) 1: 248-447.

Ubick, D. and V.D. Roth. (1973). Nearctic Gnaphosidae including species from adjacent Mexican states. American Arachnology 9(suppl. 2): 1-12. Walckenaer, C.A. (1842). Histoire naturelle des Insects. Aptères. Paris, 2: $1-549 \mathrm{pp}$.

Wang, X.P. (2002). A generic-level revision of the spider subfamily Coelotinae (Araneae, Amaurobiidae). Bulletin of American Museum of Natural Historty 269: 1-150.

Wanless, F.R. (1978). A revision of the spider genus Portia (Araneae: Salticidae). Bulletin of the British Museum Natural History (Zoology) 34: 83124

Wanless, F.R. (1979). A revision of the spider genus Brettus (Araneae: Salticidae). Bulletin of the British Museum Natural History (Zoology) 35: 183-190.

Wanless, F.R. (1980). A revision of the spider genera Asemonea and Pandisus (Araneae: Salticidae). Bulletin of the British Museum Natural History (Zoology) 39: 213-257

Wanless, F.R. (1984). A revision of the spider genus Cyrba (Araneae: Salticidae) with the description of a new presumptive pheromone dispersing organ. Bulletin of the British Museum Natural History (Zoology) 4.7: $445-481$ 
Wirth, V. von. (1991). Eine Revision der Gattung Ornithoctonus Pocock 1892 (Araneida: Theraphosidae: Ornithoctoninae). Arachnologischer Anzeiger (Affalterbach) 12: 5-8.

Wunderlich, J. (1979). Linyphiidae aus Nepal, III. Die Gattungen Caviphantes Oi 1960 und Lessertiella Dumitrescu \& Miller 1962 (Arachnida: Araneae). Senckenbergiana biologica 60: 85-89.

Wunderlich, J. (1986). Spinnenfauna gestern und heute: Fossile Spinnen in Bernstein und ihre heute lebenden Verwandten. Quelle \& Meyer, Wiesbaden. Wunderlich, J. (1987). Die Spinnen der Kanarischen Inseln und Madeiras: Adaptive Radiation, Biogeographie, Revisionen und Neubeschreibungen. Triops Verlag, Langen, West Germany.

Wunderlich, J. (1992). Die Spinnen-Fauna der Makaronesischen Inseln: Taxonomie, Ökologie, Biogeographie und Evolution. Beiträge für Araneologie 1: 1-619

Yoshida, H. (1978). On some Formosan spiders (1). Atypus 71: 21-28.

Yoshida, H. (1993). Notes on Argyrodes xiphias Thorell, 1887 (Araneae: Theridiidae) from South East Asia. Acta arachnologica, Tokyo 42: 83-85. Yoshida, H. (2002). A revision of the Japanese genera and species of the subfamily Hadrotarsinae (Araneae: Theridiidae). Acta arachnologica Tokyo 51: 7-18.

Yu, L.M. \& D.X. Song (1988). A revision of the Chinese spiders of the family Lycosidae (Araneae). Sinozoology 6: 113-121.

Zabka, M. (1981a). Salticidae from Kashmir and Ladakh (Arachnida, Araneae). Senckenbergiana biologica 61: 407-413.

Zabka, M. (1981b). New species of Yaginumaella Prószyn'ski 1976 and Helicius Prószyn'ski 1976 (Araneae: Salticidae) from Bhutan and Burma. Entomologica basil. 6: 5-41.

Zabka, M. (1985). Systematic and zoogeographic study on the family Salticidae (Araneae) from Viet-Nam. Annales zoologici Warszawa 39: 197485 .

Zabka, M. (1988). Salticidae (Araneae) of Oriental, Australian and Pacific regions, III. Annales zoologici Warszawa 41: 42 1-479.

Zabka, M. (1990). Salticidae from the Nepal and Bhutan Himalayas. Genera Pancorius Simon 1902, Plexippus C.L. Koch 1846, and Pseudamycus Simon 1885 (Arachnida: Araneae). Senckenbergiana biologica 70: 161178

Zhang, J., M.S. Zhu \& D. Song (2005). Revision of the spider genus Hamataliwa Keyserling from China (Arnaeae: Oxyopidae). Zootaxa 1017: $1-17$.

Zhang, C., M.S. Zhu \& D.X. Song (2006). A review of the Chinese species of the genus Eriophora (Araneae: Araneidae). Acta arachnologica Sinica 15: 1-13.

Zhu, M.S., D.X. Song \& J.X. Zhang (2003). Fauna Sinica: Invertebrata Vol. 35: Arachnida: Araneae: Tetragnathidae. Science Press, Beijing, vii+418pp.

\section{ACKNOWLEDGEMENTS}

We are grateful to Ansie Dippenaar, Peter Jaeger, Pekka T. Lehtinen, Paula E. Cushing, Rajashekhar Patil, Sudhi Kumar A.V. and other arachnologists whose assistance in preparing the checklists is invaluable. Other important persons and institutes we are grateful to are: Ms. Sally Walker (for constant encouragement and support to the study and conservation of all lesser-known creatures); Dr. N.I. Platnick and his team (for the excellent compilation and continuing work on The World Spider Catalog, without which this paper would have been near impossible); Aravind Ventakesan, Praveen and B. Ravichandran (for their painstaking numbering, renumbering and proofreading); and finally our crucial supporters The Rufford Small Grants Programme of The Rufford Maurice Laing Foundation, Fauna and Flora International and Cleveland Metropark Zoo (for supporting and funding the field work on theraphosid spiders in India, which has indirectly helped in preparing this checklist).

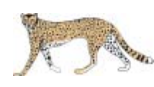

\section{ZOO OUTREACH ORGANISATION}

\author{
requires a Copy Editor
}

The candidate should be a graduate in biology with interest in the subject as well as with a good working, reading and writing knowledge of English. Preferably, the candidate should be skilled in the language, be able to proofread, have an eye for details, be meticulous, and with an apetite to read a lot. Candidate will be based in Coimbatore.

The candidate will be required to work on various aspects of the education programmes of ZOO, with primary focus on language editing of the monthly Journal and Magazine and regular editing of the various education packets produced by the organisation. Candidates with a keen interest to work hard for the sake of conservation and wildlife and with no ambitions of a 9-5 lifestyle need apply with a curriculum vitae, a letter of interest (1-2 pages typed), two references and complete communication details to zoocrew@vsnl.net

The candidate's experience, dedication and willingness to work will determine the pay scale after a 3-month probation.

\section{Ashoka Trust for Research in Ecology and the Environment}

\section{Conservation Education (Program Officer)}

This position is located in Bangalore.

The candidate should have a minimum Masters Degree in natural or social sciences and demonstrated experience in conservation education. Must be willing to travel to other states in South India when required. The job primarily entails coordinating the rural conservation education initiatives of ATREE in the southern region. $\mathrm{S} /$ he also will be required to facilitate urban education outreach in Bangalore. Must have good written and oral communication skills. Fluency in Kannada/Tamil is an added advantage.

Candidates are welcome to visit www.atree.org for more information. ATREE fosters diversity and gender equity at the work place. Thus women and persons from underprivileged groups are especially encouraged to apply. Applications will be accepted till the position is filled. The position applied for should be mentioned in the subject line of the email/superscribed on the envelope. Please submit a CV and an expression of interest (2-3 pages) with the names, phone numbers and of at least 2 references to the following email id kalpana@atree.org 